\title{
Structural investigation of the histone chaperone complex FACT using genetically encoded crosslinkers in Saccharomyces cerevisiae
}

\author{
Dissertation \\ for the award of the degree \\ "Doctor rerum naturalium" \\ of the Georg-August-Universität Göttingen \\ within the doctoral program "Molecular Biology" \\ of the Georg-August University School of Science (GAUSS) \\ submitted by \\ Christian Hoffmann \\ from Altdöbern
}

Göttingen 2014 


\section{Thesis Committee}

Prof. Dr. Heinz Neumann, Applied Synthetic Biology Group, University of Göttingen

Dr. Wolfgang Fischle, Max Planck Research Group Chromatin Biochemistry, Max Planck Institute for Biophysical Chemistry

Prof. Dr. Steven Johnsen, Clinic for General, Visceral and Pediatric Surgery (Translational Cancer Research), University Medical Center Göttingen

\section{Members of the Examination Board}

Prof. Dr. Heinz Neumann, Applied Synthetic Biology Group, University of Göttingen Dr. Wolfgang Fischle, Max Planck Research Group Chromatin Biochemistry, Max Planck Institute for Biophysical Chemistry

Prof. Dr. Steven Johnsen, Clinic for General, Visceral and Pediatric Surgery (Translational Cancer Research), University Medical Center Göttingen

\section{Further members of the Examination Board}

Prof. Dr. Holger Stark, Research Group 3D Electron Cryo-Microscopy, Max Planck Institute for Biophysical Chemistry

Dr. Hans Dieter Schmitt, Research Group Membrane Transport in Yeast, Max Planck Institute for Biophysical Chemistry

Prof. Dr. Detlef Doenecke, Dept. of Molecular Biology, Göttingen University Medical School

Date of oral examination: 01.12 .2014 
"Everything existing in the Universe is the fruit of chance and of necessity." - DEMOCRITUS

taken from 'Chance and Necessity' by Jacques Monod (1970)

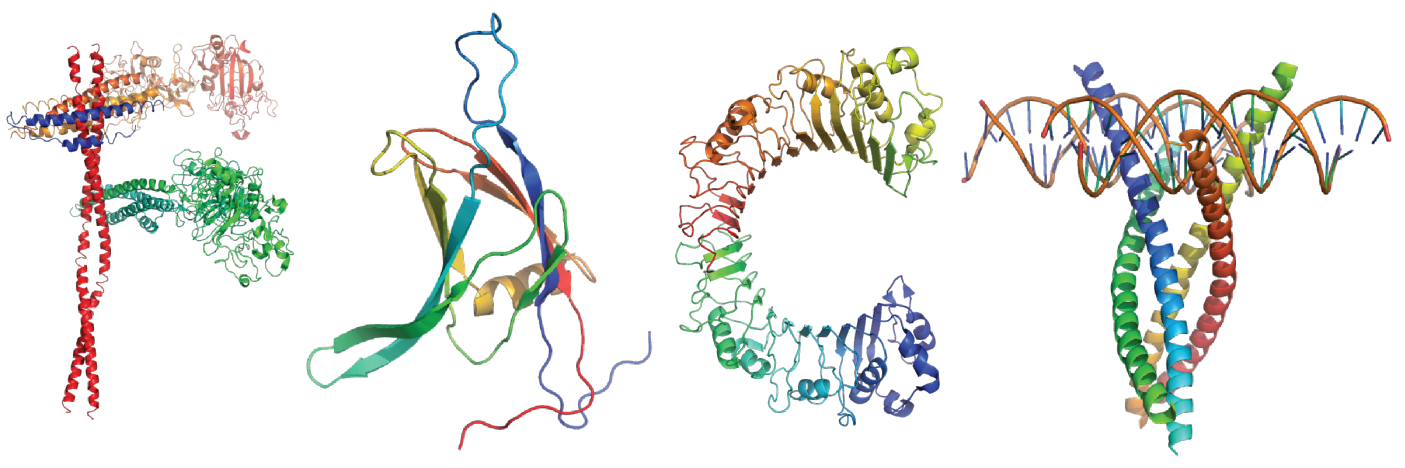

pdb: $F(2 X N X)$ A (2ENJ) C (1WWL) T (1FOS) 



\section{Contents}

Summary .................................. xi

\begin{tabular}{lll}
\hline & Introduction & 1
\end{tabular}

1.1 General introduction - Structural biology . . . . . . . . . . . . . . 1

1.2 DNA, histones and chromatin dynamics . . . . . . . . . . . . 2

1.2.1 DNA as the storage for genetic information . . . . . . . . . 2

1.2.2 Histones and nucleosomes - the fundamental building blocks 3

1.2.2.1 Nucleosome positioning .............. 5

1.2.2.2 Histone variants . . . . . . . . . . . . . 6

1.2.2.3 Post-translational modifications . . . . . . . . . 8

1.2.3 Chromatin and nucleosome dynamics . . . . . . . . . . . . 10

1.2.3.1 Transient DNA breathing and alternative nucleosome core structures . . . . . . . . . . . . . . . 10

1.2.3.2 ATP-dependent remodeling of nucleosomes . . . . . 12

1.3 Histone chaperones - guards and guides . . . . . . . . . . . . . . . . . . . . . . . . 13

1.3 .1 A general overview . . . . . . . . . . . . . . . . 13

1.3.2 Structural implications of histone chaperones. . . . . . . . . 15

1.3.3 Histone chaperones during replication . . . . . . . . . . 17

1.3.4 Histone chaperones during transcription . . . . . . . . . . . 19

1.4 The histone chaperone complex FACT . . . . . . . . . . . . . 20

1.4.1 Structure and functional implications of the FACT complex

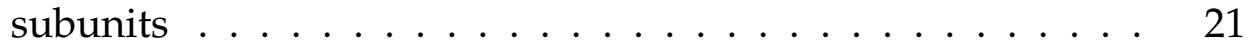

1.4 .2 Mechanistic insights into FACT activity . . . . . . . . . . . 24

1.4.2.1 The dimer eviction and global accessibility model . 25

1.4.3 FACT during transcription, replication and DNA repair . . . 26

$\begin{array}{lll}\text { 1.4.3.1 Roles during transcription initiation and elongation } 26 & 26\end{array}$

1.4.3.2 Functions of FACT in replication . . . . . . . . . 27

1.4.3.3 The role of FACT in DNA repair . . . . . . . . . . . . 28

1.5 Genetic code expansion - a versatile tool for genetically encoding unnatural amino acids . . . . . . . . . . . . . . . . . . . . . . 29

1.5.1 The Genetic code expansion principle . . . . . . . . . . . . . 29 
1.5.2 Recent applications of the Genetic code expansion system . . 31

1.5.3 Using genetic code expansion for the incorporation of lightinducible crosslinkers into proteins. . . . . . . . . . . . . 32

1.5.3.1 The photo-crosslinkers: 4-Benzoyl-L-phenylalanine (pBPA) and 4-Azido-L-phenylalanine (AzF) . . . . . 32

1.5.3.2 Incorporation of UV inducible crosslinker amino acids in Escherichia coli . . . . . . . . . . . . . . . . 34

1.5.3.3 Incorporation of pBPA and pAzF in and Saccharomyces cerevisiae and higher eukaryotes for UV-inducible crosslink-

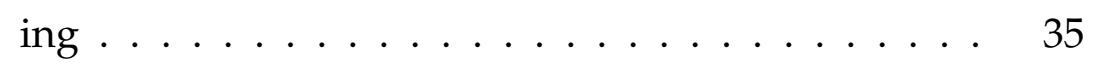

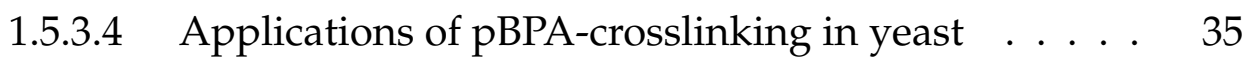

1.5.3.5 Photoaffinity crosslinking in higher eukaryotes and for the analysis of DNA-protein interactions . . . . . 36

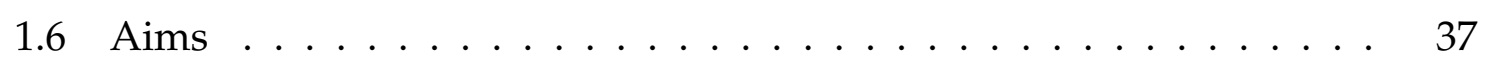

2 Material and Methods $\quad 39$

2.1 Material and equipment . . . . . . . . . . . . . . . . . . 39

$2.1 .1 \quad$ Equipment, consumable supplies and chemicals . . . . . . . 39

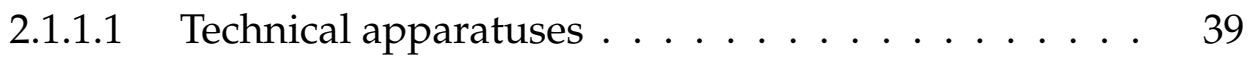

2.1 .1 .2 Laboratory utensils . . . . . . . . . . . . . 40

2.1 .1 .3 Consumable supplies . . . . . . . . . . . . . . 40

2.1.1.4 Chemicals. . . . . . . . . . . . . . . . . . 41

$2.1 .2 \quad$ Frequently used solutions and buffers . . . . . . . . . . . 42

2.1.2.1 Solutions . . . . . . . . . . . . . . . . 42

2.1 .2 .2 Media and supplements $\ldots \ldots \ldots$. . . . . . . 43

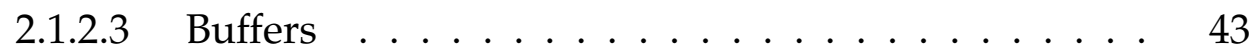

2.1.2.4 Antibiotics . . . . . . . . . . . . . . 43

2.1.2.5 SDS-PAGE and Western Blot buffers and solutions . 44

2.1 .3 Used Kits . . . . . . . . . . . . . . . . . . . . . 44

2.1 .4 Strains . . . . . . . . . . . . . . . . . . 44

2.1.4.1 Bacteria . . . . . . . . . . . . . . . 44

2.1 .4 .2 Yeasts . . . . . . . . . . . . . . . . . 45

2.1.5 Used chromatography material and columns: . . . . . . . . 45

2.1 .6 Antibodies . . . . . . . . . . . . . . . . . 46

2.1 .7 Enzymes and DNA Ladder . . . . . . . . . . . . . . . 46

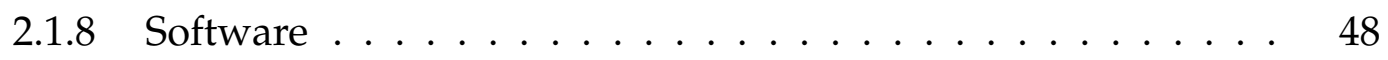

2.1 .9 Vectors $\ldots \ldots \ldots \ldots \ldots \ldots \ldots$ 
2.2 Methods . . . . . . . . . . . . . . . . . . . . . . 49

2.2 .1 DNA methods. . . . . . . . . . . . . . . . . . . . . . . . . . 49

2.2.1.1 Isolation of genomic DNA from Saccharomyces cerevisiae 49

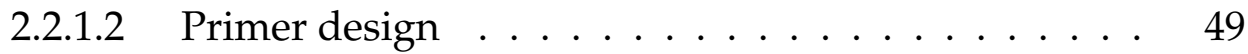

2.2 .1 .3 PCR amplification . . . . . . . . . . . . . . . . . . . 49

2.2.1.4 Quikchange mutagenesis PCR: . . . . . . . . . . 50

2.2 .1 .5 Restriction digests $\ldots \ldots \ldots \ldots$. . . . . . . . . 51

2.2.1.6 Ligation of DNA molecules . . . . . . . . . . . 51

2.2.1.7 Transformation of bacteria . . . . . . . . . . . . 52

2.2 .1 .8 Minipreparation of plasmids . . . . . . . . . . . 52

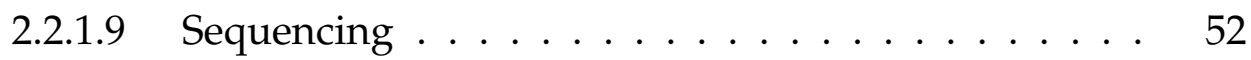

2.2.1.10 Agarose gelelectrophoresis . . . . . . . . . . . 53

2.2.1.11 Extraction of DNA from agarose gel. . . . . . . . . 54

2.2.1.12 Molecular cloning - Plasmid creations . . . . . . . 55

2.2 .2 Protein methods . . . . . . . . . . . . . . . . . . 60

2.2 .2 .1 TCA precipitation . . . . . . . . . . . . . . . 60

2.2.2.2 SDS Polyacrylamide electrophoresis of proteins . . . 60

2.2 .2 .3 Western blot and detection . . . . . . . . . . . . . 61

2.2.2.4 Protein expression and purification of yNhp6a from Escherichia coli . . . . . . . . . . . . . . 62

2.2.2.5 Protein expression and batch-purification of ctPob3 and ctPob3 $\triangle$ D467-G571 from Escherichia coli . . . . . 64

2.2.2.6 Protein expression and batch-purification of hImportin- $\alpha$ from Escherichia coli . . . . . . . . . . . . . . . . 65

2.2.2.7 Protein expression and batch-purification of ctPob3 pBPA mutants from Escherichia coli . . . . . . . . . 65

2.2.2.8 Immunoprecipitation of Pob3:9myc crosslink samples 66

2.2 .3 Chromatin methods . . . . . . . . . . . . . . . . . 66

2.2.3.1 Reconstitution of Xenopus laevis histone dimers, tetramers and octamers . . . . . . . . . . . . . . . 66

2.2.3.2 Native Polyacrylamide electrophoresis and Nhp6a binding assays $\ldots \ldots \ldots \ldots \ldots \ldots \ldots$

2.2 .4 Yeast methods and crosslinking . . . . . . . . . . . . . . 68

$2.2 .4 .1 \quad$ Yeast culturing . . . . . . . . . . . . . . . . . . 68

2.2.4.2 Standard yeast genetic manipulation . . . . . . . . . 69

2.2.4.3 Subcellular fractionation of yeast cells . . . . . . . 70

$2.2 .4 .4 \quad$ Live cell imaging of yeast . . . . . . . . . . . . 70 
2.2.4.5 In vivo crosslinking using pBPA in Saccharomyces cerevisiae . . . . . . . . . . . . . . . . . . 71

2.2 .4 .6 In vitro crosslinking of proteins . . . . . . . . 72

$\begin{array}{lll}3 & \text { Results } & 73\end{array}$

3.1 In vivo crosslinking studies of FACT in Saccharomyces cerevisiae . . . . 73

3.1.1 Detection of plasmid-borne Spt16 and Pob3 harboring different protein tags by western blot . . . . . . . . . . . . 73

3.1.2 In vivo pBPA crosslinking of Pob3 is UV- and position-dependent 74

3.1 .3 Optimization of the crosslinking approach . . . . . . . . 76

3.1.3.1 The influence of crosslinking time on the formation of the Pob3 S500 crosslink product . . . . . . . . 77

3.1.3.2 Fluorescent and standard chemiluminescent western blot detection . . . . . . . . . . . . . . 78

3.1.3.3 Comparison among sample preparation techniques: Yeast Protein Extraction Reagent, TCA precipitation and crude extract preparation . . . . . . . . . 79

3.1.3.4 Crosslinking experiments in protease deficient yeast strains decrease protein degradation $\ldots . . . .79$

3.1.4 Crosslinking screen of the yFACT complex using the Spt16 and Pob3 amber suppression libraries . . . . . . . . . . . 81

3.1.4.1 Scanning of the FACT complex with the genetically encoded UV-inducible crosslinker pBPA in Saccharomyces cerevisiae . . . . . . . . . . . . 82

3.1.4.2 Characterization of the amber suppression libraries using temperature sensitive yeast strains . . . . . . 84

3.2 The in vivo interactions between FACT and histones . . . . . . . . . . 88

3.2.1 Characterization of the acidic C-terminal tail serine 500 of Pob3 88

3.2.1.1 Identification of the S500 crosslinking product using molecular weight shift assays . . . . . . . . 88

3.2.1.2 Localization of the formed crosslinking products at S500 . . . . . . . . . . . . . . . 90

3.2.1.3 Comparison among two genetically encoded UVinducible crosslinker amino acids: pBPA and pAzF . 91

3.2.1.4 Differential crosslinking pattern of the Pob3 CTD to the H2A-H2B dimer . . . . . . . . . . . . . . . . 94

3.2 .2 Interactions of the FACT complex to other histones . . . . . . 96

3.2 .3 Immunoprecipitation of pBPA crosslink adducts . . . . . . . . 100 
3.3 In vitro analysis of FACT interactions using the complex homologue from Chaetomium thermophilum . . . . . . . . . . . . . . . . . . . . . 101

3.3.1 Expression of ctPob3 containing the genetically encoded UVinducible crosslinker pBPA in Escherichia coli . . . . . . . . . . 102

3.3.2 In vitro crosslinking studies of ctPob3-pBPA to reconstituted histone dimers and tetramers . . . . . . . . . . . . . . . . 102

3.3.3 Purification of recombinantly expressed yNhp6a from Escherichia coli and its impact on in vitro crosslinking studies of ctPob3pBPA mutants to reconstituted mononucleosomes . . . . . . . . 107

3.4 Biological relevance of the acidic terminal tail of Pob3 . . . . . . . . . 111

$3.4 .1 \quad$ Identification of a novel nuclear localization signal . . . . . . 112 3.4.1.1 Prediction of the C-terminal NLS using bioinformatics 112 3.4.1.2 Analysis of the putative Pob3 NLS by live cell imaging 113

3.4.2 The effect of the Pob3 CTD-NLS on the histone H2A-H2B dimer interaction . . . . . . . . . . . . . . . . . . 113

3.4.2.1 Interaction studies between recombinantly expressed human importin- $\alpha$ and the Chaetomium thermophilum Pob3 homologue . . . . . . . . . . . . . . . 113

3.4.2.2 Competing crosslinking studies between histone and importin- $\alpha$ binding . . . . . . . . . . . . . 119

3.4.3 Deletion of the acidic residues of Pob3-CTD in Saccharomyces cerevisiae ......................... 121

3.4.4 Influence of the genomic Pob3-CTD deletions on yeast viability

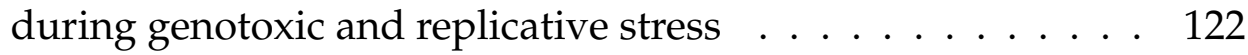

$\begin{array}{llr}4 & \text { Discussion } & 127\end{array}$

4.1 Conclusions and perspectives . . . . . . . . . . . . . . . 142

\begin{tabular}{ll}
\hline Bibliography & 145
\end{tabular}

\begin{tabular}{ll}
\hline Acknowledgements & 170
\end{tabular}

$\begin{array}{lll}5 & \text { Appendix } & 173\end{array}$

\begin{tabular}{ll}
\hline List of Figures & 185
\end{tabular}

\begin{tabular}{ll}
\hline List of Tables & 188
\end{tabular}

6 Declaration in Lieu of Oath and Curriculum Vitae 191 



\section{Summary}

Chromatin is a highly dynamic nucleoprotein structure that stores the eukaryotic genetic information. Histones are the essential chromatin proteins that form the core octamers around which DNA is compacted within the nucleus. These small proteins regulate all aspects of chromatin biology. Histone chaperones act as guards and guides of histones and are implicated in the regulation of transcription, replication and DNA-repair. In order to understand how histone chaperones achieve this variety of functions, it is important to structurally characterize the interplay between histone chaperones and their binding partners. Most structural and functional data exists from solution studies, where their implications in vivo are indirect. Only under physiological conditions can we truly begin to delineate the dynamics and interplay between chromatin and associated proteins. In this regard, methods need to be developed to address the in vivo characterization of chromatin processes.

In this thesis, I present an innovative crosslinking approach to study the interactome of the histone chaperone complex FACT (facilitates chromatin transcription) in living yeast. Using the genetically encoded crosslinker amino acid (4-Benzoyl-Lphenylalanine) pBPA at nearly two hundred different positions, I map the interactions of FACT at a single amino acid resolution, in vivo. This highly reproducible crosslinking approach reveals putative interaction surfaces for a diverse set of suggested FACT binding partners. Using this assay I show that the acidic C-terminal domain (CTD) of the FACT complex subunit Pob3 interacts with the histones $\mathrm{H} 2 \mathrm{~A} / \mathrm{H} 2 \mathrm{~B}$ in a defined manner in vivo, although this domain is predicted to be structurally disordered. Furthermore, I characterize a novel nuclear localization signal at the very end of the Pob3-CTD. Thus, in the case of the FACT complex, the acidic CTD has both a role in the nuclear transport and in the histone binding. Furthermore, my observations provide evidence for a functional role of the acidic domain during replication. Based on the data presented in this thesis I can suggest that acidic domains present on many histone chaperones act as putative binding platforms for the interaction with histones in vivo. 



\section{Introduction}

\subsection{General introduction - Structural biology}

Structural biology has led to tremendous achievements during the last century. These include the structural determinations of single globular proteins or DNA over multisubunit complexes up to fully assembled molecular machineries such as the high resolution crystal structure of the eukaryotic ribosome from yeast ${ }^{1 / 2}$. Structures detail atomic coordinates and are a rich resource elucidating and deducing mechanistical information about proteins. They aid in the improvement of chemical drug design, support evolutionary theories and are often the starting point for subsequent experiments in molecular biology. The major structure determination methods include X-ray crystallography, NMR (nuclear magnetic resonance) spectroscopy, Cryo-electron microscopy and tomography and small-angle $X$-ray scattering (SAXS). Since structural data sets are mainly obtained through in vitro methods, its interpretation remains challenging in the context of true physiological conditions in a crowed cellular environment of living organisms 3 345. These implications can affect individual protein properties such as folding, interactions and kinetics, thus, may influence the entire simplified mechanistical view gained from in vitro experiments. Limitations of some structure determination techniques arise from biological samples which are highly dynamic or contain predicted intrinsically disordered domains which may additionally challenge structural analysis.

Therefore, I want to use an in vivo crosslink assay with genetically encoded UVinducible crosslinker amino acids in living yeast (introduced in section 1.5.3). This crosslinking method would allow me to elucidate the interactome of a protein in a living organism. My research is focused on the histone chaperone complex FACT (facilitates chromatin transcription, section 1.4). Histone chaperones are guards and guides of histones (section 1.3) and are implicated in many DNA-related processes such as transcription, replication and DNA repair ${ }^{6}$. Thus far, there is no complete threedimensional structure of the entire FACT complex available, although individual domains have been structurally characterized. My in vivo crosslink approach strives to gain new structural insights into the FACT complex in its physiological environ- 
ment and may corroborate already existing structural information.

\subsection{DNA, histones and chromatin dynamics}

\subsubsection{DNA as the storage for genetic information}

In all living organisms genetic information is stored in the sequences of the chemical material deoxyribonucleic acid (DNA). Through its inheritance and change during evolution it has given rise to the plethora of life as we know it on earth. In 1869 Johann Friedrich Miescher, in an effort to elucidate the biochemical composition of lymphocytes, isolated an unknown substance from nuclei which he named "nuclein"19. This marked the first time that the substance we know as DNA was isolated. Nonetheless, it took almost eight decades until the importance of the deoxyribonucleic acid became apparent. The key experiment which identified a DNA as responsible for the inheritage of genetic information, was performed by Oswald T. Avery and colleagues in 1944. Their experiments were based upon the famous Grif-

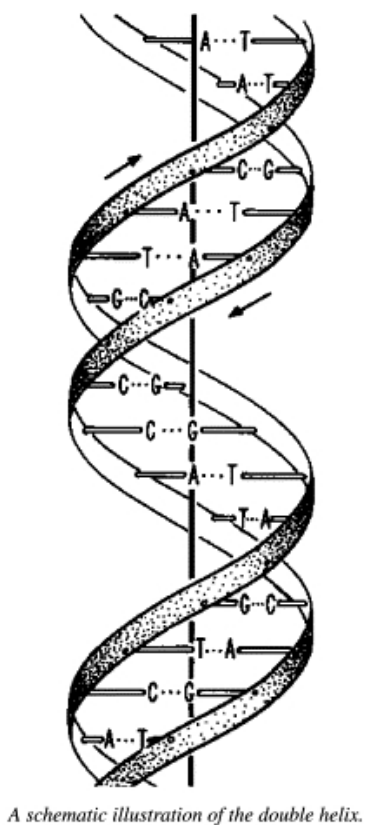

Figure 1.1: Schematic illustration of the DNA by James Watson (taken from Klug, 200478) fith pneumococcus experiment from 1928 where a mixture of a heat-inactivated strain and a non-virulent strain could regain virulency discovering the "transformation principle" in bacteria 10 . Avery and colleagues expanded the experimental setup and tested the "chemical nature of the substance" responsible for the transformation principle. They showed that the transformation principle is susceptible to crude tissue extracts containing "deoxyribonucleodepolymerase" activity and therefore identified DNA as the carrier of genetic information 11 . Nine years later the first structure of the DNA double helix was solved by Watson and Crick ${ }^{12}$ with the aid of $X$-ray crystallography data from Rosalind Franklin (figure 1.1; for a review see Klug, 20047).

Nowadays, DNA is used as a general term that refers to the DNA double helix, which consists of two strands of deoxyribonucleic acid. Each strand harbors a sugarphosphate backbone comprised of deoxyribose linked to one of the four nucleobases by a glycosidic bond 13 . The nucleobases are classified as the purines, adenine or guanine, and the pyrimidines cytosine and thymine by their ring structure. Hydro- 
gen bonds between the nucleobases adenine and thymine or guanine and cytosine provide the so called Watson-Crick base pairing in a DNA double strand. Sterically, DNA can adapt three different major conformations: The A-DNA, B-DNA and ZDNA, where the B-form is the major conformation found in physiological conditions.

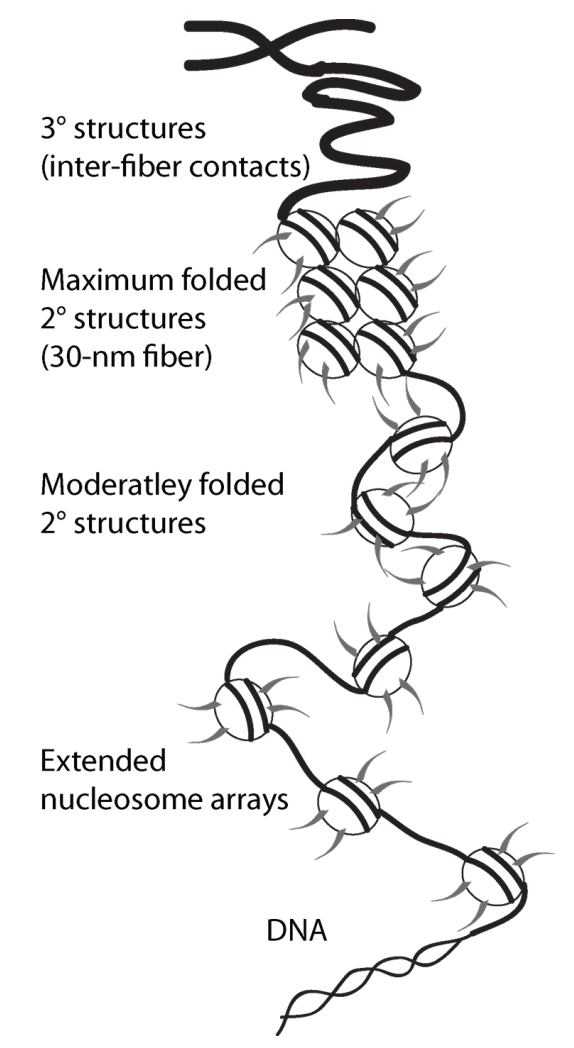

Figure 1.2: Schematic illustration of the hierarchical DNA compaction (modified from Tamara L. Caterino and Jeffrey J. Hayes, 2007 $\frac{14}{\text {, }}$

The average length of a base pair is known with $0.34 \mathrm{~nm}$ along the strand in the double helix 12 . Thinking of the human genome as one long stretch with $3.08^{*} 10^{9}$ base pairs $\frac{15}{}$, it would have an fullextended length of roughly 2 meter in a human diploid cell, although a typical eukaryotic cell has a size of only 10-100 $\mu \mathrm{m}$. Therefore, DNA needs to be packaged and highly condensed in several hierarchical layers to overcome this physical barrier. In eukaryotes compaction is achieved by wrapping the DNA double helix around a histone protein core. This basic building unit, the nucleosome core particle, lines up like beads on a string and allows for higher compaction states of the DNA molecule over intermediate fibers up to the most compact form, the mitotic chromosome (figure 1.2. . On the one hand compaction and formation of higher order structures provides physical stability but on the other hand it also limits excess to the DNA sequence and therefore the genetic information which is important for all cellular processes. This complex interplay between stability and dynamics needs to be working in chorus with processes such as transcription, translation and DNA repair.

\subsubsection{Histones and nucleosomes - the fundamental building blocks}

In Eukaryotes, histones are a group of small basic proteins which are essential for DNA compaction. They were first described by Albrecht Kossel together with protamines in $1928^{16}$. Although their primary sequence is not highly conserved among them they share a common structural fold, referred to as the histone fold (for following structural details $\frac{17118}{}$ ). This motif is comprised of three alpha helices connected by two linker turns resulting in an helix-turn-helix-turn-helix motif (figure 
1.3A). Antiparallel arrangement of the long central $\alpha 2$ helix and the resulting L1/L2 interactions allows the formation of homo- or heterodimers in solution (figure $1.3 \mathrm{~B}$ ). A second common feature are their flexible $\mathrm{N}$-terminal tails which are a target for many post translational amino acid modifications.

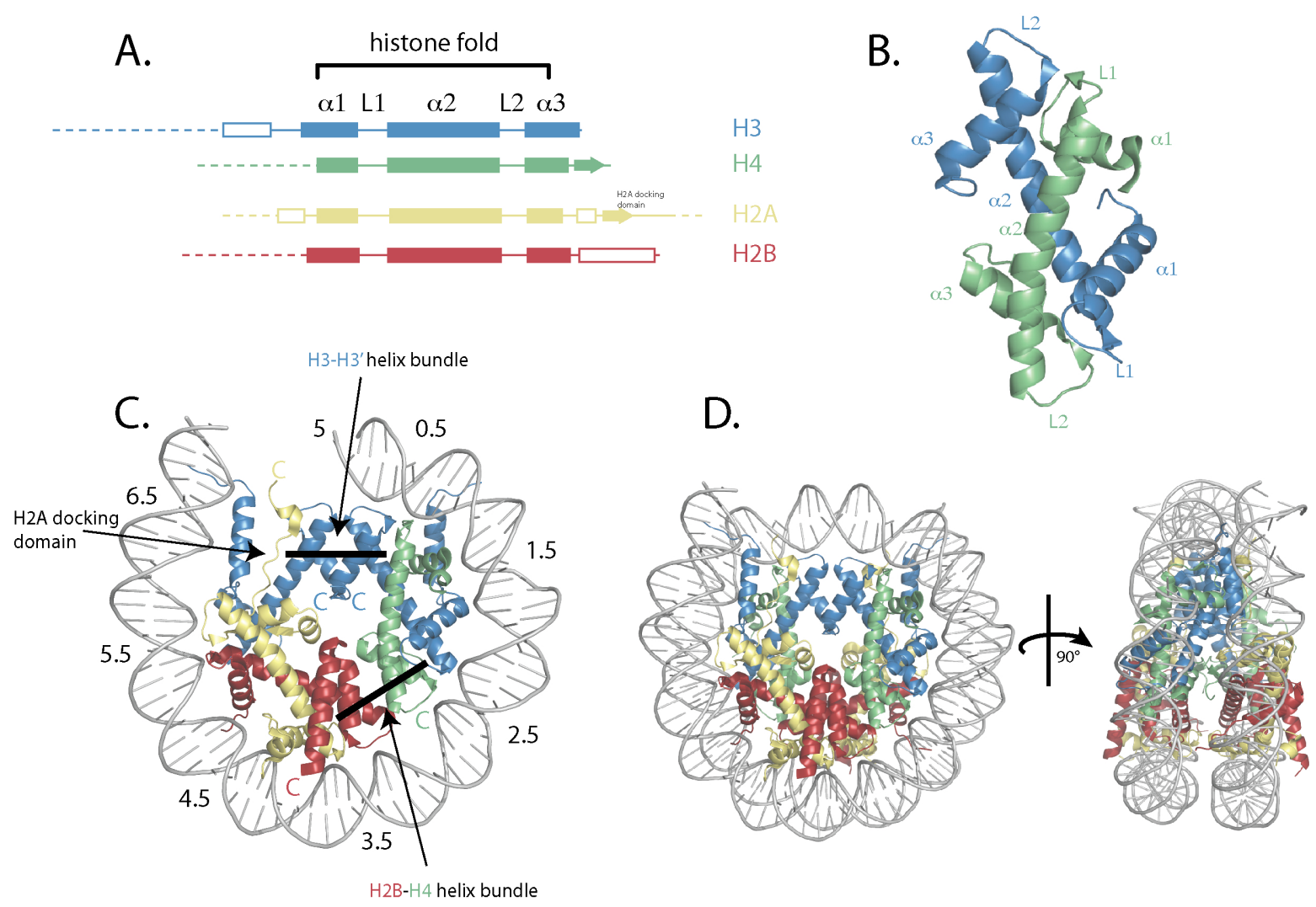

Figure 1.3: Histones, their fold and organization forming the nuclear core particle (modified after Luger et al., 2001 18) - A. Schematic representation of the four core histones, $\mathrm{H} 3, \mathrm{H} 4, \mathrm{H} 2 \mathrm{~A}$ and H2B. The histone fold is depicted and structural elements are indicated $(\alpha$ : helix, L: linker/turn, dashed: flexible histone tails). B. Structure of the histone fold of a H3-H4 dimer extracted from the nucleosome core particle structure (pdb: 3AV1; C. and D.). C: Structure of half the NCP showing 73bp DNA. Octamer stabilizing interactions, the fourhelix bundles and the H2A docking domain are indicated. Numbers are indicating the protein-DNA interaction sites formed by the histone fold dimers in terms of SHL units (superhelix location zero, relative to the central base pair which is falling on the dyad; each DNA turn increases the SHL by one). D: Structures of the NCP from SHL $-7-+7$ (pdb: 3AV1)

Two copies of each of the four core histones, H2A, H2B, H3 and H4 form an octameric protein core. Around this protein core, $\sim 145-147 \mathrm{bp}$ of DNA is wrapped in 1.65 turns of a left-handed DNA superhelix forming the basic structural chromatin unit - the nucleosome core particle (NCP). The histone core is held together by the formation of two distinct types of four-helix bundles. The stable formation of two H3-H4 dimers, forming a histone H3-H4 tetramer, is achieved by the H3-H3' four helix bundle at the entry/exit point of the DNA (symmetry dyad). This bundle 
is comprised of the H3- $\alpha 2$ and H3- $\alpha 3$ helix of each H3-H4 dimer forming stable interactions. The second type of helix bundle is not stable under physiological conditions: The H2B-H4 helix bundle between $\alpha 2$ and $\alpha 3$ helixes from H2B and H4 links the histone tetramer to one half of the histone H2A-H2B dimer. The C-terminal docking domain of $\mathrm{H} 2 \mathrm{~A}$ which interacts with the other half of the tetramer further stabilizes the histone core. The histone fold determines the direct interactions of the histone octamer core with the DNA. Each dimer has three interaction motifs, two formed by the L1/L2 loop alignment and one from the $\alpha 1 / \alpha 1$ helix structures. They are organizing 27-28 bp of DNA per dimer. Hydrogen bonds and hydrophobic interactions occur mainly between side-chain residues or main-chain nitrogens and the DNA backbone. Especially, arginin residues are interacting with the minor groove of the DNA. Ultimately, the octamer organizes the DNA at 14 independent binding sites at minor grooves of the DNA superhelix without a single DNA base pair contact. The passage of the $\mathrm{H} 2 \mathrm{~A}$ and $\mathrm{H} 3$ tails is allowed through aligned minor groves between the DNA spiral as a result of a $0.3 \mathrm{bp}$ overwind per turn of the DNA superhelix.

For the formation of higher order chromatin structures (figure 1.2) a linker histone is deposited most probably close to the dyad axis. Linker histones posses an evolutionary conserved globular domain and do not posses a histone fold. They can promote inter-nucleosome interactions and are important for chromatin condensation and decondensation (for a review ${ }^{19}$ ).

\subsubsection{Nucleosome positioning}

The absence of direct histone-nucleobase interactions raised the question of nucleosome positioning and the influence of the DNA sequence. Crystallographic data 17 implies that the sequence has a secondary effect since AT-rich regions can adopt more likely a narrow minor grove conformation as compared to GC-rich regions which are bending more likely in the major grove 20 . Hence, stronger arginine interactions with the minor grove occur in the presence local of AT-base pairing sequences 1721 . Furthermore, recent ChiP-Seq data, allowing for static genome-wide nucleosome mapping, revealed a non-random distribution of histones with a distinct nucleosomefree or depleted region $(\mathrm{N}(\mathrm{F} / \mathrm{D}) \mathrm{R})$ at the promoter which is followed by a distinct positioned (+1) nucleosome and further series of positioned nucleosomes throughout the gene body (see figure 1.4 ; for a review 22 ).

One of the first high resolution studies revealing a certain distinct nucleosome positioning pattern was done in yeast by analyzing 2278 nucleosomes over $482 \mathrm{~kb}$ DNA region 24 . Moreover, Segal and colleagues are proposing "a genomic code for 


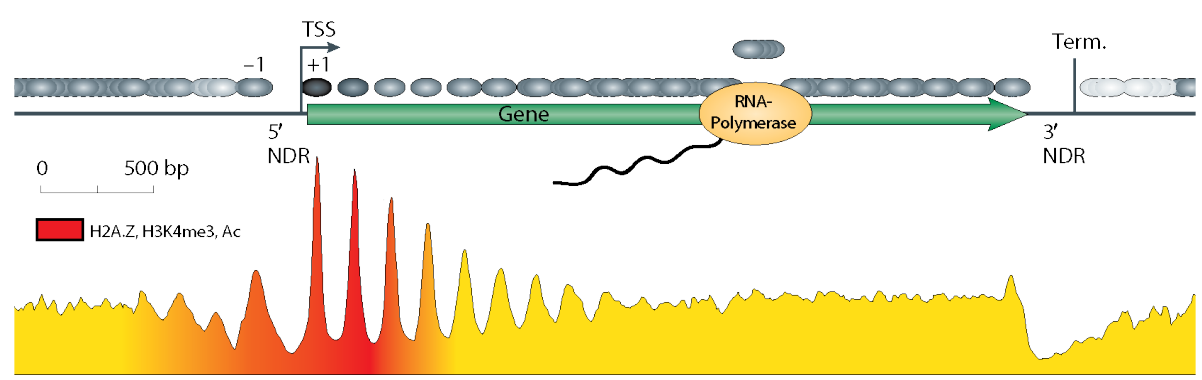

Figure 1.4: Nucleosome occupancy at genes in yeast (modified after Jiang and Pugh et al., 200922, reproduction ${ }^{23}$ ) - Schematic illustration as a consensus sequence of nucleosome occupancy is shown relative to the transcription start site (TSS). The nucleosome depleted regions are indicated at the $5^{\prime}$ and $3^{\prime}$ of the ORF are indicated. The $5^{\prime} \mathrm{NDR}$ is enclosed by the $(-1)$ and $(+1)$ nucleosome. Subsequently, phased nucleosomes are represented by distinct peaks, followed by fuzzy distributed nucleosomes spread throughout the gene body. Red colour is indicating the distribution of specific histone specific hallmarks, H2A.Z and post-translational modifications.

nucleosome positioning"25. Their model was deduced from aligned nucleosome bound sequences of different species and found enriched AA/TT/TA dinucleotides with a 10 bp periodicity of the nucleosomal DNA implying their enrichment in the minor groove of the DNA. Their computational approach was proven experimentally by Kaplan et al., 200926. They reconstituted nucleosomes on purified yeast DNA and compared the nucleosome distribution of these in vitro map with those generated from in vivo studies. In conclusion, their work reveals comparable nucleotide positioning preferences between in vitro and in vivo analysis as well as the establishment of the NFR at gene promoters, hence, claiming an intrinsic nucleosome positioning code. However, this is still under controversial debate ${ }^{27}$.

The proper nucleosome deposition, exchange and positioning by ATP-dependent chromatin remodelling complexes and histone chaperones is one crucial aspect of DNA accessibility, and therefore important for transcription, replication and DNA repair.

\subsubsection{Histone variants}

In addition to the four core histones, several histone variants exist, especially for histones H2A and H3. These include H2A.Z, MacroH2A, H2A.Bbd, H2A.X, H3.3 and CenH3. Their expression and deposition depends on the cellular and environmental context, apart from the deposition of nucleosomes during replication, and have an impact on the dynamics of chromatin (for reviews ${ }^{28129}$ ). For their incorporation, specialized histone chaperones or remodeling machines are needed. Several crystal structures of nucleosomes containing histone variants reveal a rather unaltered nucleosome core structure (structural overview ${ }^{30}$ ). Thus, changes in dynamics and 
stability are the main effects of histone variants.

H2A.Bbd and its mouse isoform H2A.Lap1 are found only in mammals and are testis and brain specific variants. They are shown to act as open chromatin markers 31 .

$\mathrm{H} 2 \mathrm{~A} . \mathrm{Z}$, a major H2A variant in eukaryotes, is distributed specifically throughout the genome in eu- and heterochromatin and shows local enrichment at promoter regions especially at the promoter flanking regions and the $(+1)$ nucleosome $^{29[32}$ (see also figure 1.4). In yeast, H2A.Z is antagonizing Sir2-dependent heterochromatin spreading and therefore important for maintenance of the chromatin state ${ }^{33}$. Interestingly, the crystal structure of a nucleosome containing H2A.Z shows no major differences in protein DNA-binding but revealed an extended $\mathrm{H} 2 \mathrm{~A}$ acidic patch formed by the $\mathrm{H} 2 \mathrm{~A}$ docking domain and $\mathrm{H}_{2} \mathrm{~B}^{34}$ and thereby changing the molecular surface of the nucleosome. Furthermore, the H2A.Z-H2B dimer interaction is affected by an incorporated H2A.Z variant. H2A.Z plays a crucial role for the formation of higher order chromatin structures as shown by in vitro compaction assays 35 .

The unusual vertebrate-specific $\mathrm{H} 2 \mathrm{~A}$ variant, macroH2A, possesses an additional globular domain connected to the C-term of H2A by a linker sequence ${ }^{36}$. MacroH2A is excessively found on the inactive $\mathrm{X}$-chromosome of female mammals and therefore involved in transcriptional repression 37 . As shown for H2A.Z, the dimer interaction surface is affected in nucleosomes containing macroH2 $\mathrm{A}^{38}$.

The H2A variant, H2A.X, is found in approximately $10 \%$ of the mammalian chromatin but presents the primary $\mathrm{H} 2 \mathrm{~A}$-form in yeast ${ }^{39}$. It's serine 139 phosphorylation on an additional C-terminal motif (SGD/E $\Phi$; $\Phi$ represents a hydrophobic amino acid) is the classical mark of a DNA double strand break (DBS) induced by

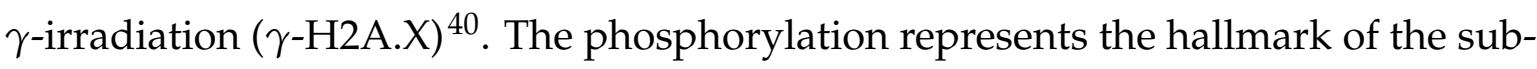
sequently following DSB-repair cascades such as homologous recombination (HR) or non-homologous end joining (NHEJ) 14 .

The major two $\mathrm{H} 3$ histone variants are the centromeric CenH3 ${ }^{42 / 43}$ (Cse4 in yeast, Cid in Drosophila, CENP-A in humans and mice) and the replication-independent variant histone H3.3. CenH3 is specifically found at the centromer and essential for kinetochore assembly $44 / 45$. Nucleosomes containing CenH3 show weakened DNA binding at the entry and exit point of the DNA due to lost base-stacking interactions $\underline{43}$.

Histone H3.3 differs only by 4 amino acids from canonical H3.2 (5 amino acid difference to the mammal specific replicative H3.1) and is enriched at active chromatin regions serving as replacement variant of the canonical $\mathrm{H} 3$ during transcription 46 . However, H3.3 is also found at telomeric heterochromatin. Deposition of this variant depends on the histone chaperone complex HIRA 47 . 


\subsubsection{Post-translational modifications}

Post-translational modifications are small chemical changes at the amino acid level. Histones carry many of the known PTMs that modify the primary histone sequence and influence thereby chromatin structure and dynamics. PTMs are established in a context-dependent fashion. Some PTMs are specific for cellular processes such as transcription or repair whereas others are influenced by the cell cycle such as replication and mitosis. PTMs are also an important branch in the context of development $\underline{48}$ and epigenetics $\frac{49}{}$, generally termed as "DNA-related regulatory mechanisms that do not involve changes in the nucleotide sequence" 50 independently of their inheritage.

Apart from rare modifications in the globular histone domains, PTMs are mainly localized to the accessible flexible N-terminal tails protruding out of the DNA and to the very C-terminal regions of the histones (figure 1.5). As mass spectrometry techniques continually improve upon resolution, novel PTMs are continuously discovered such as crotonylation $\frac{51}{}$, propionylation and butyrylation ${ }^{52}$. The main types of histone modifications are acetylation and ubiquitination of lysine, methylation of arginine and lysine, phosphorylation of serine and threonine, ADP-ribosylation of glutamate and SUMOylation of lysine (figure 1.5). The modifications are introduced by specific writer enzymes $\underline{48}$ often traveling along with molecular machineries and are read by classes of reader enzymes carrying specialized recognition domains. Examples of such reader domains include the bromo-domains for the recognition of acetylated or chromo-domains for methylated lysine ${ }^{53 \mid 54}$. Many PTM can be erased and are therefore reversible such as acetylation which is deposited by the activity of HATs (histone acetyl transfer eases) and can be erased by HDACs (histone deacetylases) 50 .

Initial observations and hypotheses of PTMs were based on the influence of the electrostatic properties of the basic histones and their interactions to the negatively charged DNA ${ }^{56}$. Hence, addition of acetylation of lysine residues would result in weakening of octamer-DNA interaction and therefore leading to more open accessible chromatin due to the reduction in the overall positive charge of the histones. Moreover, additional negative charges such as phosphorylation of serine would further destabilize octamer-DNA interactions.

In general, PTMs can either have a direct effect on chromatin structure or provide new binding interfaces for the recruitment of remodeling or transcription factors. Throughout the last decades scientist revealed that some post-translational modifications are associated with active gene transcription and chromatin such as $\mathrm{H} 3 \mathrm{~K} 4 \mathrm{me} 3^{57 / 58}$, H3K9ac ${ }^{57}$ and general H4ac whereas others are associated with repressed gene transcription and inactive chromatin such as $\mathrm{H} 3 \mathrm{~K} 9 \mathrm{me} 3$ and $\mathrm{H} 3 \mathrm{~K} 27 \mathrm{me} 3 \frac{59}{}$.

One famous example is the acetylation of $\mathrm{H} 4$ and especially that of position K16. 


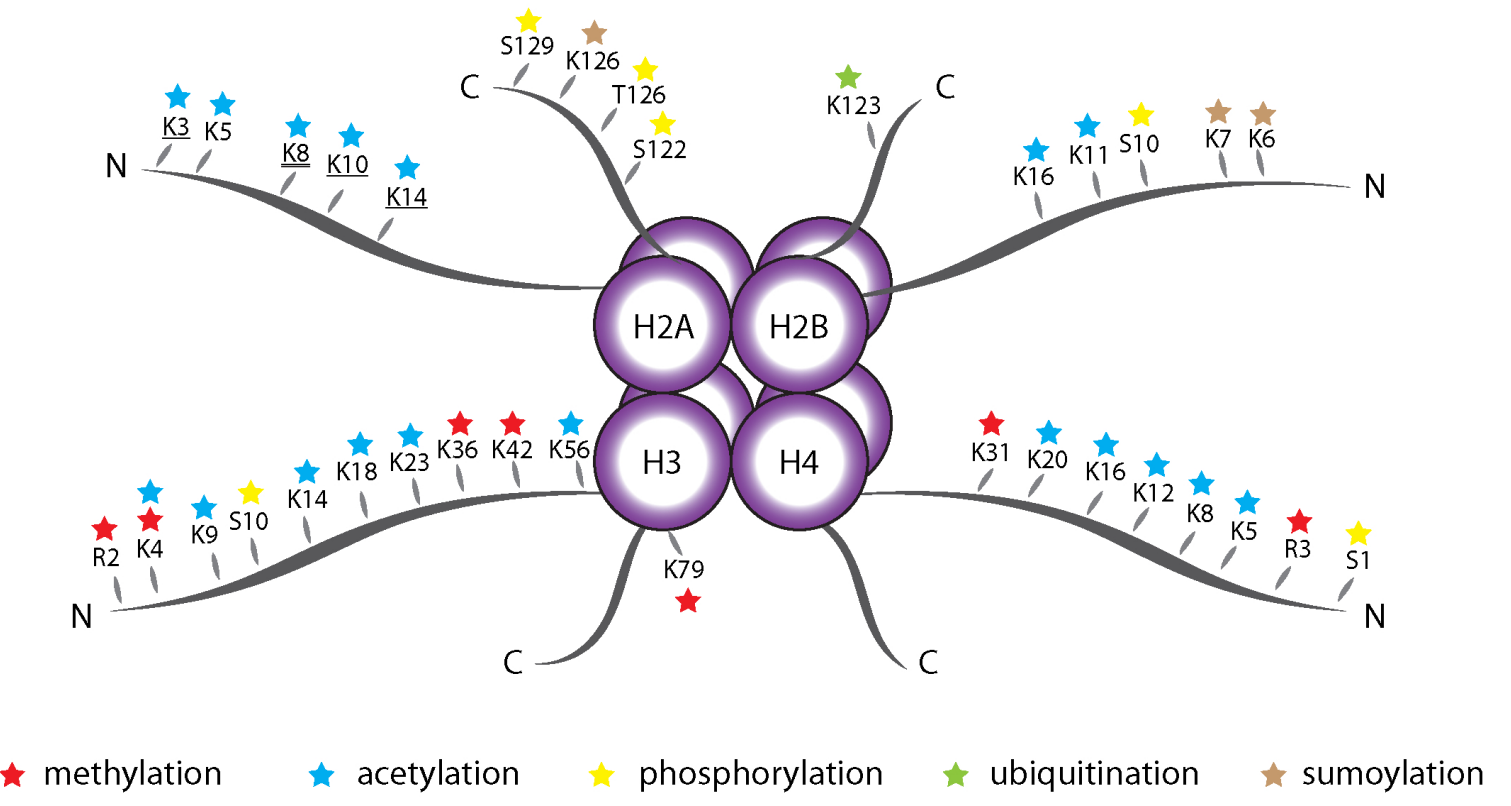

Figure 1.5: Overview of classical histone post-translational modifications in Saccharomyces cerevisiae (modified after ${ }^{55}$ ) - Schematic representation of the histone octamer core with protruding $\mathrm{N}$-terminal tails and $\mathrm{C}$-terminal parts. Underlined residues are referring to yeast Htz1 (equivalent of H2A.Z) whereas K8 acetylation is found on canonical H2A and Htz1.

For chromatin compaction, the $\mathrm{H} 2 \mathrm{~A}-\mathrm{H} 2 \mathrm{~B}$ acidic patch is crucial for nucleosomenucleosome interactions $\underline{60}$. Crystal structures reveal that adjacent nucleosomes are contacting each other by interactions of the $\mathrm{H} 4$ tail with the acidic patch 17 61/62. Recent in vivo experiments using genetically encoded crosslinker in yeast confirm these interactions in living yeast ${ }^{63}$. In 2006 , Shogren-Knaak and coworkers ${ }^{64}$ revealed the importance of the H4K16 acetylation in the context of the acidic-patch-H4-tail interaction. They showed that the acetylation inhibits interactions on the nucleosome level as well as in higher order structures such as the critically debated 30nm fiber. These works proved that this modification plays an important role for chromatin decompaction and euchromatin formation and therefore increased DNA accessibility by a single post-translational modification.

Post-translational modifications must be considered in a context outside of single independent events. The combinatorial presence of one type at different sites as well as different types all over the histone octamer gave rise to the theory of the histone code ${ }^{65}$. This code assigns patterns of PTMs to certain biological outcome such as implications on transcription, silencing or histone deposition. However, the analysis of these patterns and the crosstalk between different PTMs is challenging but crucial for the understanding of the complex interplay between chromatin structure, accessibility and gene expression $66[67$. 


\subsubsection{Chromatin and nucleosome dynamics}

Three major processes regulate DNA accessibility and are tightly interconnected to the structural variations of histones and their PTMs. First is transient DNA breathing, the second is the formation of alternative nucleosome structures and third is the remodeling of nucleosomes by ATP-dependent nucleosome remodelers. The consequence of each of these processes is DNA accessibility and they are essential for many DNA-binding proteins and molecular machineries to provide their function.

\subsubsection{Transient DNA breathing and alternative nucleosome core structures}

In 1995 Polach and Widom laid the foundation for the nature and kinetics of DNA accessibility using restriction digests on reconstituted nucleosomes with specialized DNA templates 68 . They show extensive cleavage by restriction enzymes at various sites of the nucleosome and thereby revealing the intrinsic site exposure of the nucleosomal DNA. A further study in 2005 confirmed and quantified the rapid and constant unwrapping and rewrapping of nucleosomal DNA by FRET experiments 69 . Subsequent studies determined the lifetime of the fully wrapped state with $\sim 120$ ms ${ }^{70}$. This dynamic event is termed transient DNA breathing by un/wrapping a stretch of 10 to $20 \mathrm{bp}$ of DNA from one end of the nucleosome ${ }^{71}$ (figure 1.6 left). DNA breathing affects many processes where DNA binding and accessibility are crucial such as binding of nucleosome remodelling factors and the progression of RNA-polymerase II through chromatin during transcription.

Histone variations and PTMs can influence DNA breathing. One striking example is H3K56 acetylation. This globular histone modification contacts the phosphate DNA backbone and therefore possess a potential influence on histone-DNA interactions. In 2009, Neumann and coworkers used reconstituted nucleosomes carrying the H3K56ac in combination with an end-labeled DNA FRET-pair to monitor DNA breathing in vitro (figure 1.6 left) ${ }^{75}$. They showed an increase of DNA breathing by 7-fold upon H3K56 acetylation using single-molecule FRET experiments.

In addition to DNA breathing, recent high-speed AFM studies (atomic force microscopy) revealed spontaneous splitting and complete nucleosome disassembly without the help of chromatin remodelers ${ }^{78}$.

The foundation for some alternative nucleosome structures is already encoded in the stepwise assembly and disassembly process of nucleosomes ${ }^{73}$ (figure 1.6 right). For nucleosome assembly, a H3-H4 tetramer (or in heterodimer form) is positioned onto the DNA followed by the incorporation of two H2A-H2B dimers ${ }^{79}$ (states VI. -> V. -> I.). This process can be performed in vitro by changing the ionic conditions from high to low strengths ${ }^{80[81}$. A key study, analysed the stepwise salt-dependent 

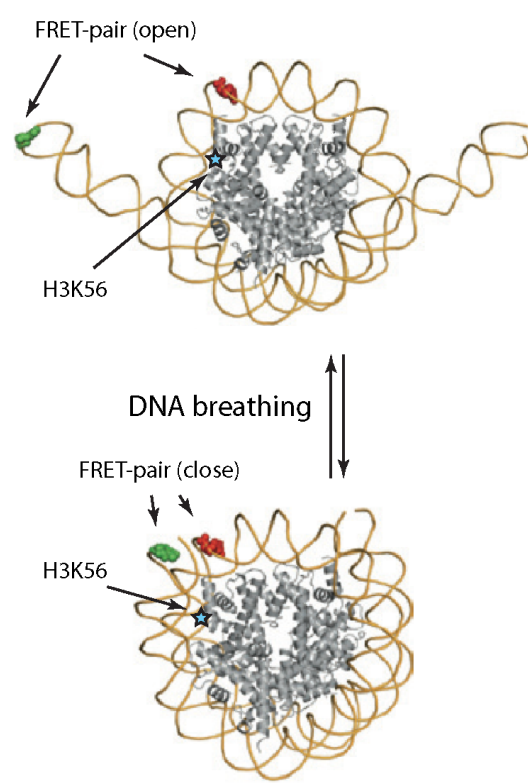
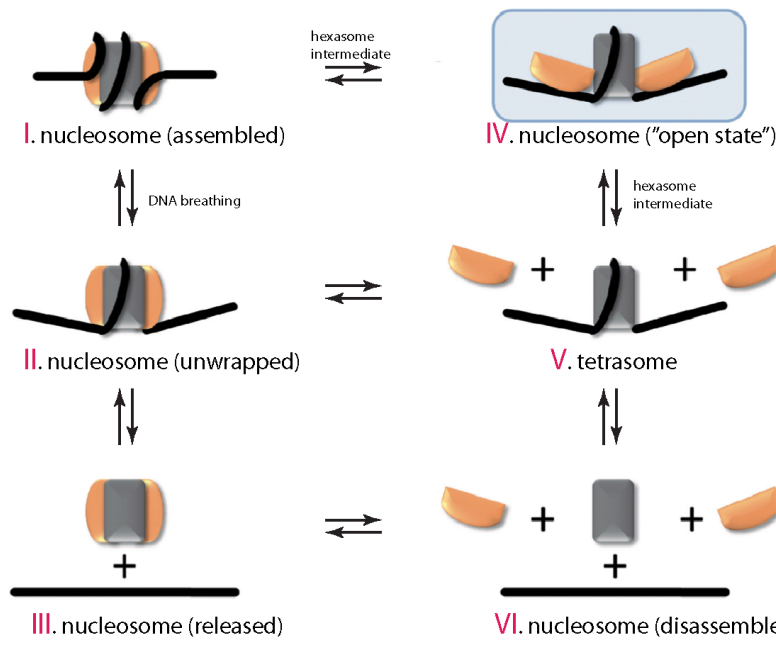

IV. nucleosome ("open state")
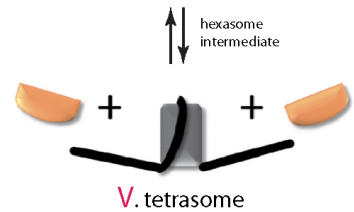

$\uparrow \downarrow$

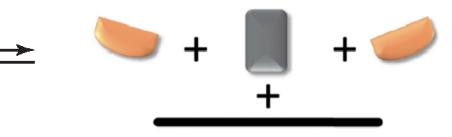

VI. nucleosome (disassembled)

Figure 1.6: Nucleosome dynamics: DNA breathing and alternative nucleosome structures (modified after ${ }^{71727374}$ ) - Left: Schematic representation of DNA breathing unwrapping the terminal 10-20 bp of the nucleosomal DNA. Figure illustrates the experimental setup analysis breathing by FRET measurements of the closed an open form. The H3K56 position is indicated and acetylation increased the breathing up to seven fold ${ }^{75}$. Right: Structural states (I. to VI.) of the nucleosome during (dis)assembly depicted as schematic representation. Stepwise assembly by positioning of the tetramer onto the DNA (VI. -> V. -> I.). Disassembly was confirmed over the a novel "open state" (I. -> V. -> VI.) ${ }^{71}$. Possible dissociation of the full octamer is depicted in state (III) 76/77]. DNA breathing (I. $<->$ II.) and implicated hexasome intermediates (lack of one H2A-H2B dimer) are depicted. Interconvertibility defined by spontaneous structural transitions (DNA breathing or opening) is assumed at many states.

assembly and disassembly processes by performing spFRET (single-pairFRET) analysis and FCS (fluorescence correlation spectroscopy) measurements by Böhm et al. in $2011^{71}$. First, they confirmed the reversed nature of the disassembly process in respect to the assembly process. Second, they provided strong evidence for an "open nucleosome state" prior to $\mathrm{H} 2 \mathrm{~A}-\mathrm{H} 2 \mathrm{~B}$ dimer dissociation whereas a separation at the dimer-tetramer occurred (state IV.). This "open state" might be a crucial interaction state for histone chaperones and remodelers assessing the nucleosome without dissociating the H2A-H2B dimer. However, hexasome formation (loss of one H2A-H2B dimer) resembles an intermediate state which was shown to be implicated during transcription elongation 82 . In vitro studies from Kireeva and co-workers revealed a loss of H2A-H2B dimer during transcription of a minimal nucleosome system by RNA-Polymerase II ${ }^{83}$. Recently, SAX data gained insights into structural properties of the hexasome and reveal a rather stable entity ${ }^{84}$. The hexasome structure is similar to the octamer structure whereas the hexasome is formed with only a 112-base-pair 
DNA fragment implying additional $35 \mathrm{bp}$ of accessible free DNA than compared to a reconstituted nucleosome with full wrapped $\sim 145-147 \mathrm{bp}$.

In general nucleosomes can turn over in vivo whereas the turnover for $\mathrm{H} 2 \mathrm{~A}-\mathrm{H} 2 \mathrm{~B}$ dimers is much faster than for H3-H4 tetramers thereby providing the possibility of alternative nucleosome structures. The differences in turnover can be explained as a cause of DNA breathing and the closer position of the dimer docking sites at the DNA entry and exit as described above. Histone turnover was beautifully demonstrated by FRAP (Fluorescence Recovery after Photobleaching) experiments using GFP-tagged histones in HeLa cells ${ }^{85}$ and subsequently by ChIP experiments in yeast ${ }^{86}$.

\subsubsection{ATP-dependent remodeling of nucleosomes}

Another way of changing chromatin structure is provided by ATP-dependent remodeling complexes which are acting on the nucleosome structures and ultimately increasing the accessibility for DNA-binding factors. Thus, clarifying their importance for processes such as transcription, replication and DNA repair (for a broad review ${ }^{87}$ ). All remodellers are highly conserved between species and possess a common ATPase domain belonging to SWI2/SNF2-family ATPase subunit 88 . The ATPase domain serves as molecular motor allowing for the disruption of octamer-DNA contacts leading to mobilisation of nucleosomes along the DNA (sliding), histone dimer eviction or exchange of histone variants. Structurally, they exist as multi-subunit complexes protein complexes with distinct functions.

The mechanistic details of nucleosome sliding for each of the remodelers is still under debate. There are three prominent models exiting 89 : The loop-propagation model is defined by the local detachment of the nucleosomal DNA leading to a loop formation which is propagated along the nucleosome ${ }^{87}$. The twist model is based on the induction of local distortions by the removal of between 4 to 7 bp of $\mathrm{DNA}^{90}$ and further propagation around the nucleosome ${ }^{91}$. Lastly, changes in the octamer structure, due to the introduced disturbances of the histone-DNA contacts by remodelers, ultimately leads to remodeling. Nevertheless, in all models the energy-dependent introduction of disturbances and relaxation of the system results in changes of DNA accessibility.

The surrounding domains of the common ATPase domain determine which family the remodeler complex falls into. These include SWI/SNF, ISWI, CHD, and INO80 87 . These domains are functionally linking to histone variants and PTMs which are important for remodeller recruitment and regulation.

Bromo-domains recognize acetylated histones such as the SWI/SNF or INO80 family. For example, the yeast Bdf1 (member of SWR1 complex and INO80 family) 
recognizes H3K14ac which is likely to influence the deposition of H2A.Z histone variants in the targeted nucleosomes ${ }^{92193}$. Other remodelers such as members of the CHD family contain chromo-domains and are closely connected to methylated histones such the active chromatin mark H3K4me3 ${ }^{94}$. Activities of remodelers can also be affected by histone variants. This is beautifully demonstrated by PapamichosChronakis and coworkers in 2011. They show that the INO80 complex catalyses the removal of mislocalized H2A.Z maintaining the proper H2A.Z chromatin distribution in concert with the H2A.Z-depositioning SWR1 complex ${ }^{95}$ (see figure 1.4 for H2A.Z distribution at the promoter region). Another example for an H2A.Z effect on a ATP-dependent remodelers is provided by Goldman et al., 2010. They showed an increased nucleosome remodeling activity of members of the ISWI family using extensive in vitro assays 96 .

\subsection{Histone chaperones - guards and guides}

\subsubsection{A general overview}

In 1978 Ron Laskey and coworkers described the isolation of a roughly $29 \mathrm{kDa}$ acidic protein from unfertilised Xenopus laevis egg homogenates with remarkable properties 97 . They showed the formation of nucleoprotein complexes of this protein with DNA and further revealed that it promotes nucleosome assembly without the requirements of magnesium ions or ATP at physiological conditions. Since a mixture of purified DNA and histones typically precipitates at low ionic strength, they concluded "that the role of the protein we (they) have purified is that of a 'molecular chaperone' which prevents ionic interactions between histones and DNA" and further that it "could prevent nonspecific ionic interactions and allow only selected specific interactions to occur" "97. With their functional discovery of the protein Nucleoplasmin 98 a new field of molecular biology, the class of molecular chaperones and specifically histone chaperones, was born.

Histone chaperones are a functional class of proteins involved in several histone related processes such as deposition onto the DNA, exchange of histone variants, nuclear import and maintaining a storage pool of histones (for broad reviews $\frac{699}{\text {; }}$ see figure 1.7). They can be seen as histone escorts at all stages of the histones cellular life especially helping in the sequential assembly and disassembly of nucleosomes.

Their presence is essential for many cellular chromatin related processes where chromatin structure has to be changed for processes such as transcription, replication and DNA repair. Furthermore, chaperones and their malfunction have recently be seen in a broader context, for example, their influence in development and pathogen- 

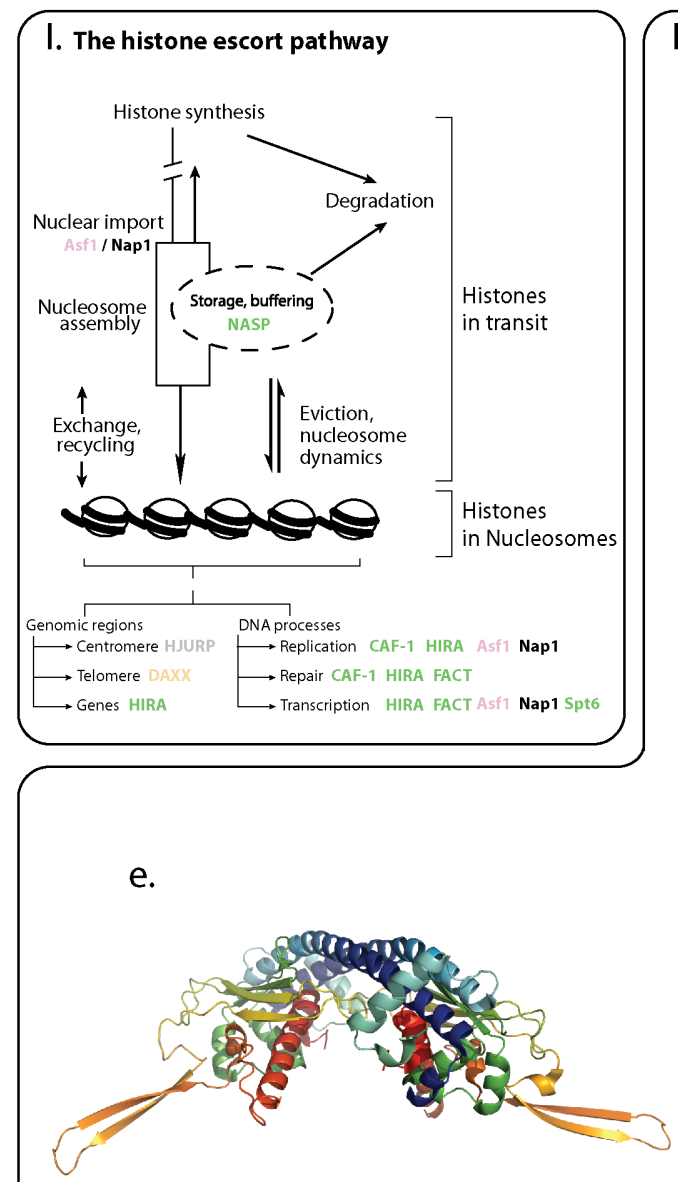

Nap1 dimer

\section{Structural studies}

a.

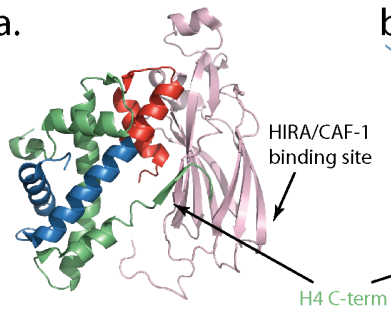

H3-H4-Asf1

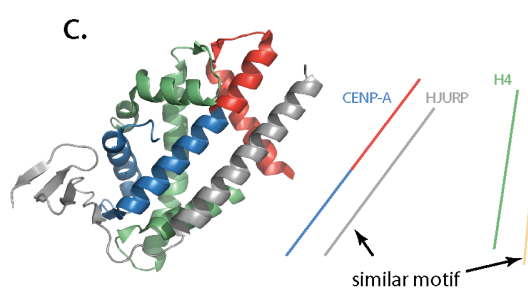

CENP-A-H4-HJURP

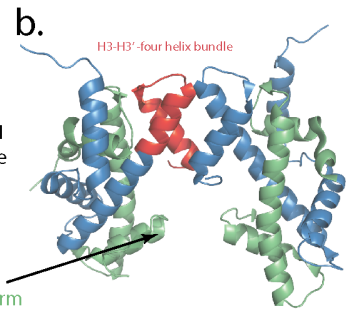

$\mathrm{H} 3-\mathrm{H} 4$ tetramer

d. f.

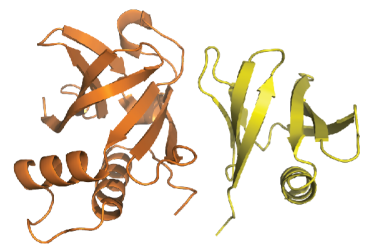

Rtt106

Ret

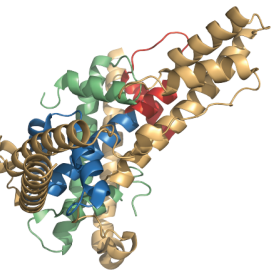

H3-H4-DAXX

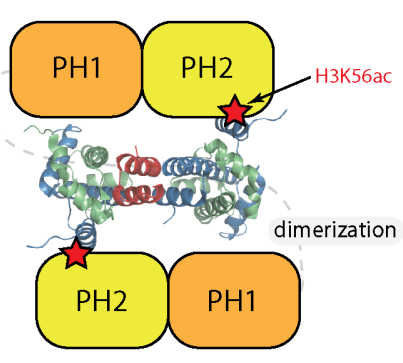

Rtt106 - purposed H3-H4 tetramer model

Figure 1.7: The histone chaperone escort pathway with selected structural studies (modified after ${ }^{699}$ ) - I. Schematic representation of the histone escort pathway. Apart from the nucleosome assembly function of histone chaperones, they furthermore escort histones throughout there whole cellular life cycle. Their structural properties (as shown in II.) link chaperones to cellular DNA related processes and genomic regions (examples are given). II. Selection of histone chaperone X-ray crystal structures: (a.-d.) Structures of chaperones in complex with $\mathrm{H} 3-\mathrm{H} 4$ aligned after the $\mathrm{H} 3-\mathrm{H} 4$ tetramer position in the nucleosome crystal structure (shown b.; extract from pdb: 3LZO; H3-H3' interaction site in red). a: Asf1 (pink, Saccharomyces cerevisiea) in complex with a H3-H4 dimer from Xenopus laevis) (pdb: 2HUE). (c. and d.) Structures of the human chaperones HJURP (grey, pdb: 3R45) and DAXX (yellow, pdb: 4HGA) in complex with human CENP-A-H4 and H3.3-H4 respectively. The similar interaction motif of the chaperones is shown as lines whereas HJURP binds to CENP-A and DAXX to H4. e: Earmuff-structure of the H2A-H2B histone chaperone Nap1 dimer (pdb: 2Z2R). f: Structure of the fungal specific H3-H3 chaperone Rtt106 as an example of a tandem PH-domain chaperone (orange and yellow). A model for binding of an entire $\mathrm{H} 3-\mathrm{H} 4$ tetramer and recognition of H3K56ac is depicted as revealed by Su et al., $2012^{100}$ 
esis $^{99}$. Generally, they can be defined as ATP-independent histone-binding proteins which ultimately alter the chromatin landscape but are not stable components of the final product 101 .

Histone chaperones can be classified into 3 families that are dependent upon their associated histone pair: H2A-H2B, H3-H4 or mixed-form chaperones. They can be further categorized by the histone variant. For example, CAF-1 (chromatin assembly factor) ${ }^{102}$ and HIRA (histone regulator $\left.\mathrm{A}\right)^{103}$ are both $\mathrm{H} 3-\mathrm{H} 4$ chaperones (discussed later). However, using immunoprecipitation assays of epitope-tagged histone $\mathrm{H} 3$ variants in HeLa cells, Tagami and coworkers revealed that CAF-1 is specific for $\mathrm{H} 3.1-\mathrm{H} 4$, the replication-dependent histone variant whereas HIRA is an exclusive H3.3-H4 chaperone representing the replication-independent variant ${ }^{47}$. Thus, revealing the concept of binding preferences which already implies the chaperones preferred cellular function. Another example is the histone chaperone HJURP (Holliday junction recognition protein; Scm3 in yeast) with its specialised function of depositing CENP-A-H4 (Cse4 in yeast) at the centromer 104105. Thus being cellcycle-specific and furthermore exclusively acting at a specific genomic loci. However mixtures within one family are possible: NASP (nuclear autoantigenic sperm protein) which is responsible for histone supply and turnover ${ }^{106}$ or Asf1 (antisilencing function 1) ${ }^{107}$ can act with H3.1-H4 and H3.3-H4 ${ }^{47}$. Moreover, H2A-H2B/H3-H4 interfamily complexes exist such as the FACT (facilitates chromatin transcription) $\frac{82}{8}$ or Nap1 (nucleosome assembly protein 1) $108 \mid 109$. Although all histone chaperones posses an in vitro assembly property, their in vivo function and histone preference may be different. Histone chaperones can cross-talk between each other revealing redundant actions. One example was elucidated by Kaufman and colleagues showing that single deletions of either CAF-1 or HIRA in yeast are tolerated whereas double deletions show severe growth defects 110 . Redundancy can also occur within a chaperone such as the observed redundancy of the Spt16 N-terminal domain and the Pob3 middle domain of the FACT complex ${ }^{111}$.

\subsubsection{Structural implications of histone chaperones}

Many histone chaperones such as Asf1, Nap1, Rtt106, Vps75, DAXX, Spt16 and Pob3 contain acidic regions or patches comprised out of glutamic and aspartic acid often found at the C-terminus (for a schematic overview ${ }^{112}$ ). Therefore they are implicated in neutralizing the basic charge of the histones $\frac{101}{}$. However, conservation of these regions between species can differ as shown for the fungi Asf1 acidic patch which is absent in other eukaryotes 113 . Despite these charged commonalities, many histone chaperones possess characteristic histone binding motifs. 
Structural investigation of histone chaperones, often in complex with their associated histones, gave interesting insights into their mechanistic and functional properties (see figure 1.7, II. right). Moreover, they revealed that histone chaperones can possess quite diverse structures. The histone chaperone Asf1, one of the most conserved H3-H4 chaperones, has achieved the remarkable role as a so called "bridging" histone chaperone (figure 1.7, II. a. and b.). Molecularly, it interacts with the H3-H4 dimer at the $\alpha 3-\alpha 2$ helixes which is important for the octamer core formation by the H3-H3'-four helix bundle 114 . Therefore, it excludes H3-H4 tetramerization and does not discriminate between $\mathrm{H} 3.1-\mathrm{H} 4$ and $\mathrm{H} 3.3-\mathrm{H} 4$ since varying histone $\mathrm{H} 3$ residues are exposed (aa. 87, 89, 90) $\frac{115}{1}$. Furthermore, it contacts the C-terminal part of $\mathrm{H} 4{ }^{114}$. Interestingly, Asf1 possess one interaction site on the compact immunoglobulin-like $\beta$ sandwich fold which is important for interactions with two different histone chaperones HIRA and CAF-1 via the chaperones B-domain 116 . In higher eukaryotes two isoforms of Asf1 exist: Asf1a and Asf1b and it was shown that HIRA has a preference for Asf1a and CAF-1 for interactions with Asf1b 117 . Thus, achieving additional specialization, since CAF-1 is involved in the replication-dependent assembly depositing $\mathrm{H} 3.1-\mathrm{H} 4$ and HIRA in the replication-independent nucleosome assembly using H3.3-H4. The role of Asf1 in bridging these two pathways by providing H3-H4 dimers to both chaperones is a remarkable feature. Despite this internal histone chaperone adapter domains, many chaperones possess adapter domains linking them to cellular machineries and their appropriate "place-of-action". Exemplary, CAF-1 which interacts with the outer front site of PCNA (proliferating cell nuclear antigen) and is therefore physically linked to DNA-synthesis 118 .

The structures of the chaperones HJURP and DAXX with their specialized functions contain a conserved similar long helix motif for histone binding, while HJURP is interacting with CENP-A and DAXX with H4 (figure 1.7. II. c. and d.). A study from Elsässer et al. determined DAXX specificity to certain residues such as glycine 90 in H3.3 and glutamate 225 in DAXX ${ }^{119}$ whereas Hu and coworkers revealed that serine 68 of CENP-A provides specificity for the HJURP-CENP-A interaction 120.

The structure of the classic H2A-H2B chaperone Nap1 revealed a new structural fold ( $\alpha / \beta$-earmuff) and dimerizes via a Non-Coiled-Coil helical motif and forming a "headphone" structure. Thereby an acidic groove is build which is most probably involved in histone binding ${ }^{121}$ (figure 1.7. II. e.). Despite Nap1-related chaperones in higher eukaryotes, Vsp75 was found to be a yeast specific NAP1-related histone chaperone ${ }^{122}$. Recent studies imply a possible tetramerization of Nap1 or Vsp75 concluded from observed ring-like structures in solution by small angle X-ray scattering (SAX) and multi angle light scattering (MALS) ${ }^{123}$.

Another class of histone chaperones contain Pleckstrin homology domains (PH- 
domains) ${ }^{124}$ such as the H3-H4 chaperone Rtt106 (figure 1.7, II. f.) or the FACT complex which is discussed later in section 1.4. Interestingly, fungal specific Rtt106 is specifically recognizinging histone dimers containing an acetylation at $\mathrm{H} 3$ at lysine $\mathrm{K} 56^{100}$. In yeast, H3K56ac is abundant on newly synthesized histones ${ }^{125}$ which is promoted by the histone acetyltransferase Rtt109-Vps75 complex ${ }^{126} 127$. This modification is crucial for the assembly process since it increases the binding affinity of histone $\mathrm{H} 3$ toward CAF-1 and Rtt106 both in vivo and in vitro 128 . Thereby promoting nucleosome assembly during replication. Furthermore, it was shown to be crucial for chromatin reassembly after DNA repair ${ }^{129}$. As for Nap1 and Vsp75 a H3-H4 tetramer binding model for Rtt106 is suggested $100[130$.

\subsubsection{Histone chaperones during replication}

During replication in S-Phase the DNA has to be duplicated. Since DNA is packed into chromatin and wrapped around the nucleosomes, the nucleosomal landscape needs to be disassembled in front of the replication fork and then reassembled subsequently. Two general mechanisms are present during replication: First recycling of the parental histones and second the assembly of newly synthesised histones (for a simplified overview ${ }^{6}$; see figure 1.8).

The later process is achieved by sufficient supply with fresh histone H2A-H2B dimers and H3-H4 tetramers. The histone chaperones Nap1 and Asf1 are responsible for binding histone dimers in the cytosol and transporting them with the help of karyopherins into the nucleus 132 133/134. Asf1 supplies the major H3-H4 deposition chaperone CAF-1 with fresh H3-H4 dimers as described above. Since Asf1 can only bind H3-H4 dimers, the question of how H3-H4 tetramer formation occurs arose. Since CAF-1 is known for dimerization and can bind to a crosslinked H3-H4 tetramer, it may deposit either dimers or tetramers during replication $135[136$. Furthermore, CAF1 is physically linked to the replication machinery by interactions with PCNA 118 . In addition to CAF-1, the fungal specific chaperone Rtt106 is able to deposit H3-H4 onto the newly synthesized DNA strand most probably as tetramers indicated by the structural model ${ }^{100 / 130}$. As discussed earlier, H3K56ac is an important mark in fungi promoting nucleosome assembly by increasing the binding affinities for CAF-1 and Rtt106 ${ }^{128}$. Recent studies identified the presence of H3K56ac in humans by mass spectrometry 134 . Moreover, the HAT CBP in flies and additionally p300 in humans are responsible for acetylation of H3K56 in concert with Asf1, respectively Asf1a isoform in humans 137 . Thus proposing similar positive implications for nucleosome assembly by CAF-1 as in yeast. Despite the vague roll of H3K56ac during nucleosome assembly in higher eukaryotes ${ }^{99}$, two major common acetylations on $\mathrm{H} 4$ at lysine 


\section{Histone chaperones during Replication}

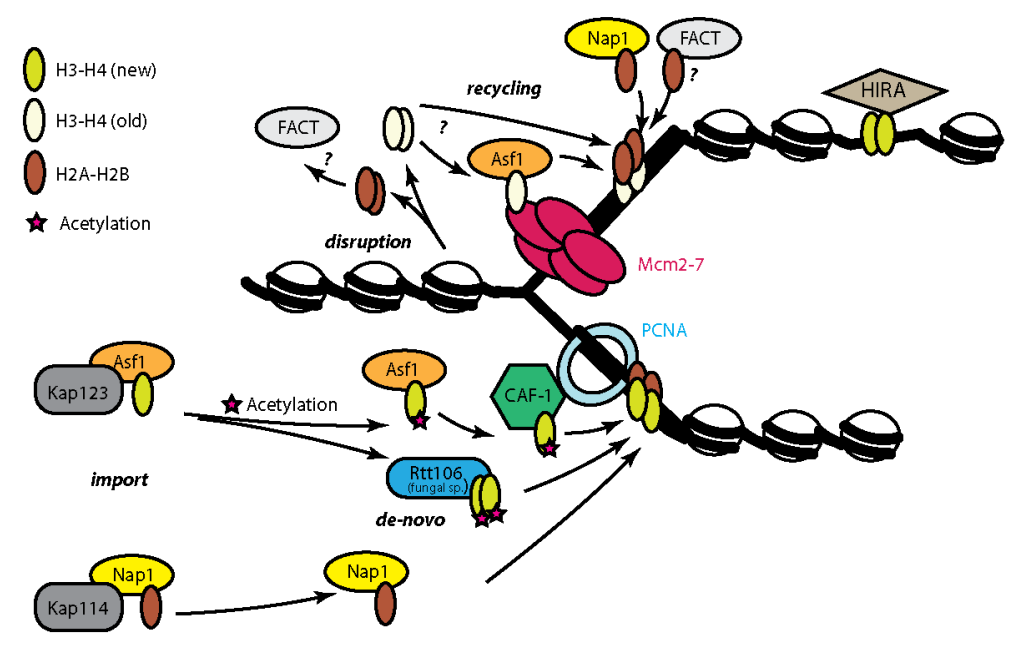

Figure 1.8: A simplified view on histone chaperones during replication (modified after ${ }^{699}$ ) - Major key players for the DNA synthesis-coupled (DSC) pathway (for details see text). The nucleosome structure is disassembled at the replication fork and histones are recycled. Asf1 is involved in depositing of old $\mathrm{H} 3-\mathrm{H} 4$ dimers and one key player for recycling. Newly synthesised histone dimers $\mathrm{H} 2 \mathrm{~A}-\mathrm{H} 2 \mathrm{~B}$ and $\mathrm{H} 3-\mathrm{H} 4$ are imported with their corresponding histone chaperone and karyopherin. Acetylation is claimed to be a general mark of the $\mathrm{H} 3-\mathrm{H} 4$ dimers. Asf1 is supplying CAF-1 with $\mathrm{H} 3-\mathrm{H} 4$ which is deposited on the newly synthesised DNA strand. A redundant role of the chaperone Rtt106 is specific for fungi. Nucleosome structures are fully assembled either with the help of the general H2A-H2B chaperone Nap1 or most likely by the FACT complex which might also be involved in recycling of parental H2A-H2B. HIRA, as a chaperone of the DNA synthesis-independent (DSI) pathway, may maintain genome integrity at improper assembled regions 131 .

5 and 12 are a general mark of newly synthesized histones found from yeast to human $133|134| 138$.

In addition to the de novo assembly of nucleosomes from newly synthesized histones, the parental histones can be recycled. In front of the replication fork H3-H4 histone tetramers and dimers are evicted. This might be aided by Asf1 since Natsume and coworkers revealed a tetramer disrupting ability of Asf1 in vitro 139 . Furthermore, Asf1 is associated through interactions with H3-H4 to the MCM2-7 helicase complex, the DNA unwinding complex at the replication fork ${ }^{140}$. This implies that Asf1 is a histone acceptor and donor at the replication fork.

Recent masspectrometry analysis, using SILAC-labeling technique for separating new from old histone species, revealed that approximately 10-20\% of the H3.3$\mathrm{H} 4$ tetramer undergo splitting events during replication-dependent nucleosome assembly, whereas most of the H3.1-H4 are unaffected and remained intact 141 . Nevertheless, the distribution of parental histones including their epigenetic PTM marks is a complex and emerging research field. 
Despite of Asf1 recycling function, the H2A-H2B dimers are most probably escorted and later reassembled by the FACT complex which is known to bind to several proteins of the replication machinery such as RPA ${ }^{142}$ or the MCM helicase complex 143 .

In general, after deposition of the $\mathrm{H} 3-\mathrm{H} 4$ tetramer, $\mathrm{H} 2 \mathrm{~A}-\mathrm{H} 2 \mathrm{~B}$ dimers are rapidly associated with the help of the chaperone Nap1. Nap1 is shown to assemble an entire nucleosome in vitro ${ }^{144 \mid 145}$. It can bind $\mathrm{H} 2 \mathrm{~A}-\mathrm{H} 2 \mathrm{~B}$ as well as $\mathrm{H} 3-\mathrm{H} 4$ in vitro, but possess a preference for $\mathrm{H} 2 \mathrm{~A}-\mathrm{H} 2 \mathrm{~B}^{108}$. However, its in vivo role is designated for the deposition of H2A-H2B dimers completing nucleosome assembly. Nap1 additionally acts in concert with ACF, an ATP-dependent remodeler of the ISWI family, to achieve evenly spaced nucleosomes after replication 146

\subsubsection{Histone chaperones during transcription}

A complex network of histone chaperones and ATP-dependent remodelers perform important actions during transcription initiation as well as elongation ${ }^{55 / 99 / 112}$. It is generally accepted that first, promoters have to be accessible for transcription (initiation), second the RNA-polymerase needs to be guided through the nucleosome structure (elongation) and third, after passage the nucleosome structure has to be reassembled to prevent cryptic transcription (for a simplified scheme of transcription elongation see figure 1.9 .

Histone chaperones during transcription

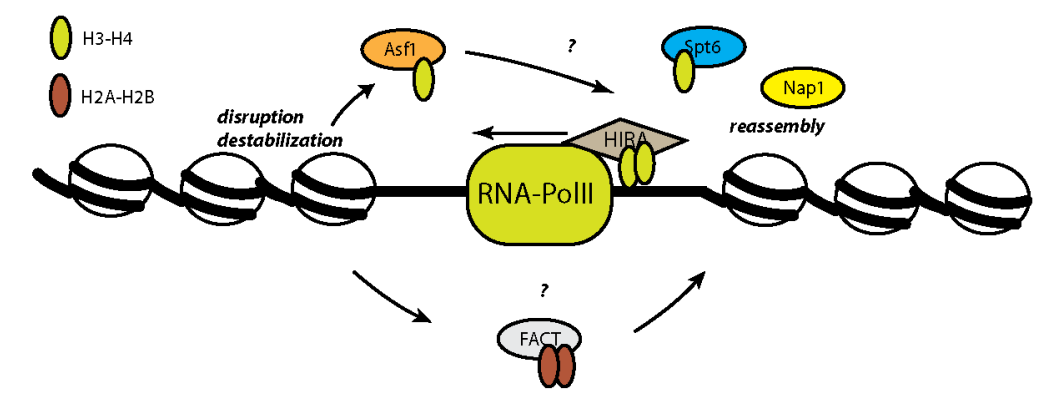

Figure 1.9: Histone chaperones during transcription elongation (modified after ${ }^{699}$ ) - Several histone chaperones are involved in promoting RNA-polymerase II progression through the gene body. They are important for the destabilization in front and reassembly of the chromatin landscape after polymerase passage ${ }^{[55] 99 \mid 112}$ (see text for details).

For the eviction of nucleosomes at promoters mainly ATP-dependent remodelers such as Swi/Snf ${ }^{147}$ respectively RSC, are involved ${ }^{148155}$. Additionally, the chaperone Asf1 has a disruptive and destabilising role on nucleosomes during initiation and elongation of transcription $149[150$. Furthermore, HIRA is shown to interact with RNA- 
polymerase II and is involved in H3.3-H4 deposition during transcription 103|131] The authors elucidated that the interaction of HIRA occurs with the initiating (Ser5phosphorylated CTD) and elongating (Ser2-phosphorylated CTD) RNA-polymerase II form. Another important elongation factor, Spt6, was described by Bortvin and Winston in yeast 151 . The chaperone binds preferentially $\mathrm{H} 3-\mathrm{H} 4$ and is implicated in histone assembly during elongation 112 . Spt6 contains a tandem SH2 domain which is important for the direct interaction with the CTD of the RNA-polymerase II ${ }^{152}$.

Several models exist for the translocation of the RNA-polymerase II through the nucleosome structure. These models and the influence of the histone chaperone complex FACT during transcription is discussed in the next chapter.

\subsection{The histone chaperone complex FACT}

The FACT complex (facilitates chromatin transcription) is an abundant essential eukaryotic ATP-independent histone chaperone being involved in several chromatin related processes, such as transcription, replication and DNA repair ${ }^{153 / 154 \mid 155 / 156}$. A haploid yeast cell harbors approximately 25.000 molecules which would roughly occupy every third nucleosome ${ }^{153}$. In lower eukaryotes such as yeast and other fungi, the complex consist of three subunits: Spt16 (Supressor of Ty 16, $120 \mathrm{kDa}$ ) initially identified in a yeast genetic screen as Cdc68 $\frac{157}{}$, Pob3 (Pol1-binding protein 3, 63 kDa) found as protein bound to a Pol1 affinity matrix together with Cdc68 158 , and the small DNA-binding HMG-box (High-mobility group) protein Nhp6 (non-histone chromosomal protein $6,11 \mathrm{kDa})^{159}$. In higher eukaryotes FACT exists as heterodimer comprised of Spt16 and SSRP1 (Structure specific recognition protein 1) 1601161 . SSRP1 is the homologue of yPob3 containing a HMG-box DNA-binding motif at the Cterminus (for a domain overview see figure 1.10). The single components of the FACT complex are highly conserved in eukaryotes ${ }^{153 / 155}$. Interestingly, Pob3 is shown to be essential in Saccharomyces cerevisiae but not in the close relative Schizzosaccharomyces pombe ${ }^{162}$.

The FACT complex possesses intrinsic chaperone activity depositing histones onto DNA for the formation of nucleosomes ${ }^{82}$. The human FACT complex was first extracted from HeLa cells revealing its ability to facilitate traveling of the RNApolymerase through chromatin templates $\frac{160}{}$, thereby unraveling one of its most famous functions during transcription. Moreover, FACT is implicated in several diverse cellular processes such as maintaining transcription by overcoming transcriptional obstacles $82\left[160 \mid 163\right.$, transcription initiation ${ }^{164}$, preventing cryptic promoter 
activation 165$] 166$, transcription termination $[167$, regulating cell cycle progression control ${ }^{168}$, mRNA export ${ }^{169}$, promoting progression of the replication complex ${ }^{170}$ and DNA repair 171 .

\subsubsection{Structure and functional implications of the FACT complex subunits}

Selected domain structures of the FACT complex which are discussed in the next paragraphs are shown in an overview figure 1.10. Thus far, there is no complete three-dimensional structure of the entire FACT complex available.

Spt16-NTD: The N-terminal domain shares structural homology with an ancient but inactive amino-peptidase fold containing the typical pita-bread fold of the Cterminal lobe (NTD-2) ${ }^{111}$. The NTD from Schizosaccharomyces pombe (PDB: 3CB6) can bind H3-H4 in vitro ${ }^{174}$. A deletion of the NTD is viable in baker's yeast but results in sensitivity to hydroxyurea 172 . Therefore, the NTD is implicated to function during replication stress signaling. Furthermore, genetic studies combining H2A-docking domain mutations, which are crucial for dimer-tetramer interactions and stability, with the NTD deletion results in loss of growth implying that the docking domain is involved in nucleosome reorganization by FACT ${ }^{111}$.

Spt16-DD and Pob-NTD/DD: The structure of the Spt16-DD domain was solved by co-crystallization with the N-terminal domain of Pob3, both from Chaetomium thermophilum 175 . This structure consists of three PH-domains, one tandem $\mathrm{PH}-$ domain from Pob3-NTD/DD and one single domain from Spt16-DD. Interestingly, they form a conserved $\beta$ sheet surface patch. Particularly immobilized ctSpt16DD was able to pull-down TAP-tagged Pol-1 which couples the dimerization domain to the replication machinery.

Spt16-MD/Pob3-MD: The structure of the yeast Spt16-MD was solved recently by Kemble et al., in 2013 176 . The Spt16-MD exhibits a tandem PH-domain architecture which binds H3-H4 in vitro and is homologue to the Rtt106 structure ${ }^{177}$ as well as to Pob3-MD 142 . In contrast to the later, Spt16-MD contains an extended $\alpha$-helix in the first PH-domain. The structure of the Chaetomium thermophilum Spt16-MD confirmed the double PH-motif and was published by the group of Ladurner 175 . They co-crystallized a chimeric ctSpt16:H2B fusion construct connected by a serineglycine linker in the presence of H2A from Xenopus laevis. Their structure contains a conserved hydrophobic C-terminal U-turn motif, lacking in the Kemble structure, 


\section{Structures of FACT}
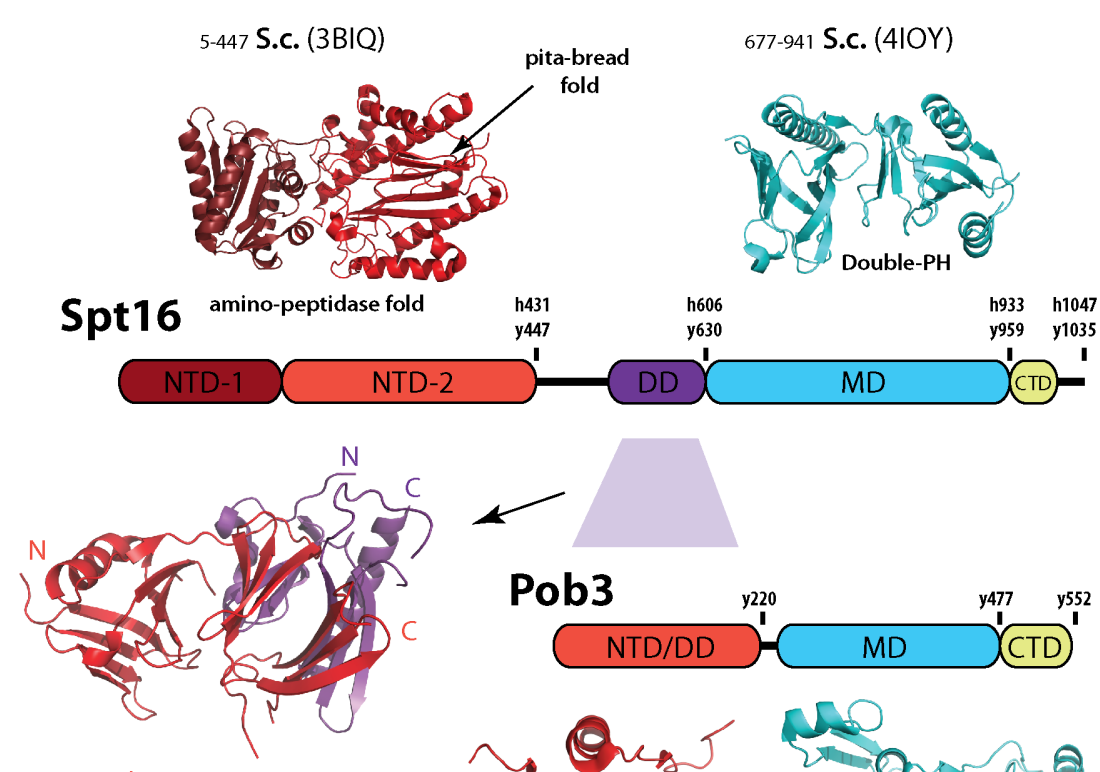

Pob3 1-185 C.t. +

Spt16 527-642 C.t. (4KHB)

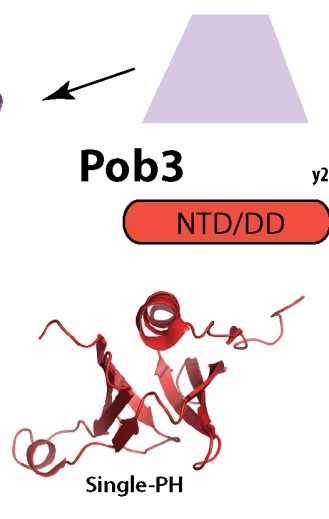

1-111 S.c. (3F5R)

677-941 S.c. (4IOY)

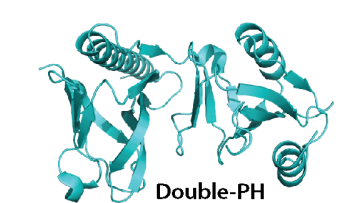

Spt16:H2B 649-944:1013-1121 C.t. : X.I. + H2A 11-102 X.I. (4KHA)
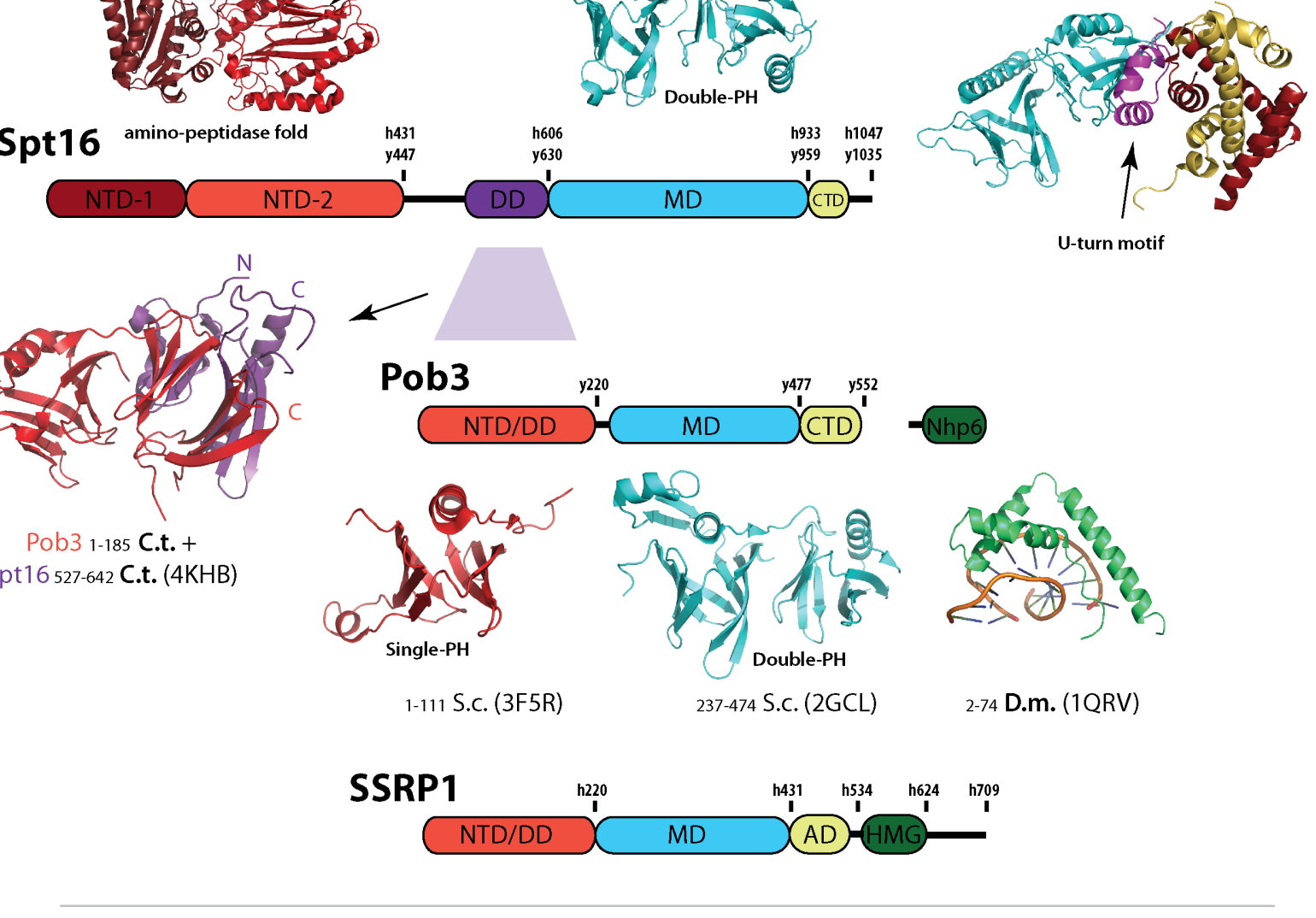

\section{Models of FACT}

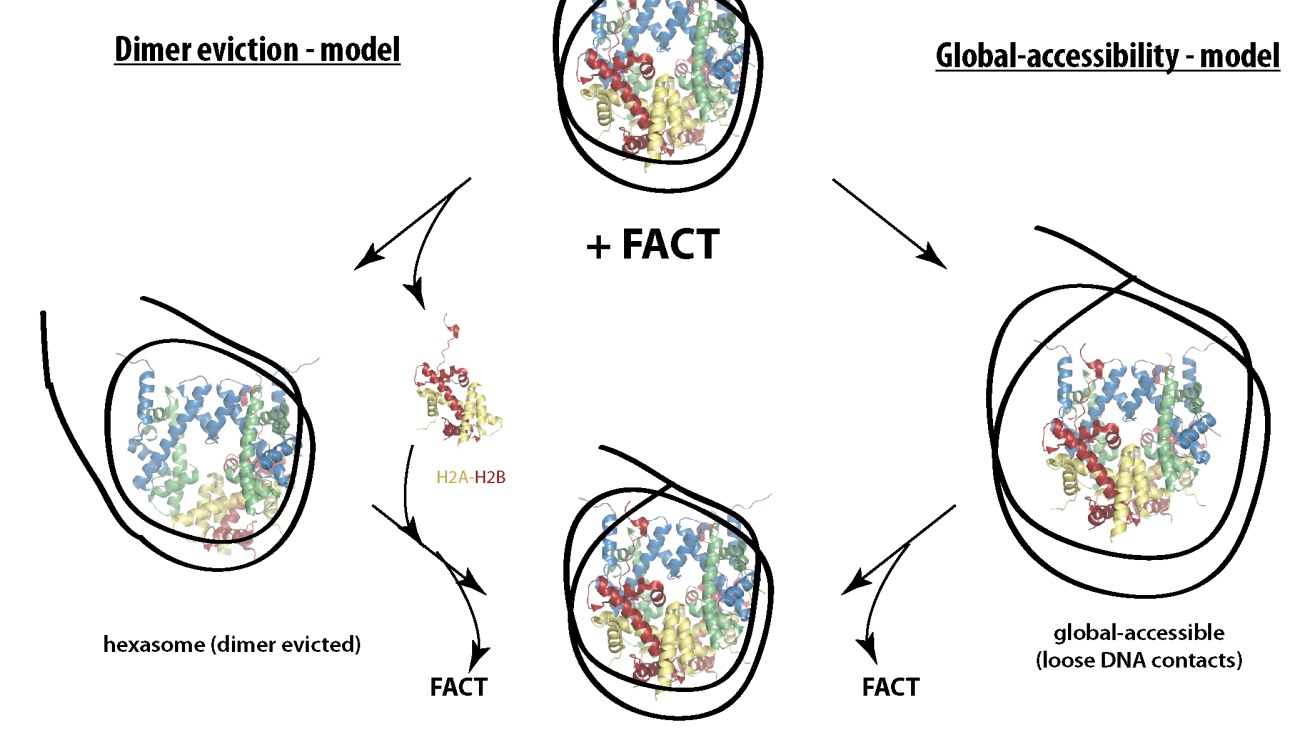

Global-accessibility - model

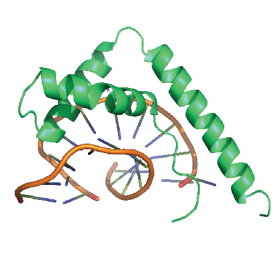

2-74 D.m. (1QRV)

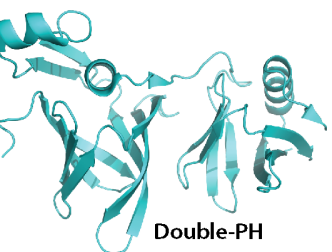

237-474 S.C. (2GCL)

Figure 1.10: Selected structures of FACT domains and models for nucleosome reorganisation (modified after $\frac{153155}{}$ ) - Caption next page. 
(Previous page.) Top: Selected structures of single FACT domains. Scheme represent the domain architecture of Spt16, Pob3 (fungi) and SSRP1 (NTD: N-terminal domain [red], DD: dimerisation domain [red/violet], MD: middle domain [cyan], CTD: C-terminal domain [yellow], AD: acidic domain, HMG [green]). Domain boundaries are given for the yeast (y) or the human (h) form based upon limited proteolysis and functional studies ${ }^{111|172| 173}$. Domains are presented as ribbon diagram from several crystal structures. PDB ID in brackets. Organism in bold: S.c. (Saccharomyces cerevisiae), C.t. (Chaetomium thermophilum), D.m. (Drosophila melanogaster, X.1. (Xenopus laevis). Numbers indicating crystallised residues. PBD 4KHB represents co-crystallisation of Spt16-DD with Pob3 NTD/DD. PDB 4KHA represents chimeric Spt16:H2B protein co-crystallised with H2A (U-turn motif for interaction with $\mathrm{H} 2 \mathrm{~B}$ is indicated). Bottom: Models for reorganisation of nucleosomes by FACT. Left: Dimer eviction model - FACT evicts a single H2A-H2B dimer leading to hexasome formation. Dimer reinsertion after cellular machineries passed (transcription, replication or DNA repair). Right: Global accessibility/non-eviction model: FACT binding induces conformational changes without eviction of a H2A-H2B dimer which remains associated with the nucleosome. Reinsertion after cellular processes are finished (see text for details, models discussed in ${ }^{153 \mid 155}$ ).

which interacts with a hydrophobic patch of the $\alpha-1$ helix of H2B. Furthermore, they confirmed the H3-H4 interactions by pull-down assays concluding that the Spt16-M domain is responsible for $\mathrm{H} 2 \mathrm{~A}-\mathrm{H} 2 \mathrm{~B}$ and $\mathrm{H} 3-\mathrm{H} 4$ binding. By an alignment of their structure with the nucleosome structure (PDB 1EZQ), they suggest a model where FACT binds initially to the N-terminal tails and progresses into the nucleosome, thereby increasing DNA breathing by reorganizing the first 30 base pairs of the nucleosomal DNA. In addition to this model, a more recent study published by Zheng et al. from $2014^{178}$ showed the importance of a highly conserved basic H2B domain in eukaryotes (yeast residues 30 to 37), called H2B repression domain (H2BHBR) domain 179 . They showed that yFACT activity is crucially dependent on the presence of the HBR domain in vitro and in vivo 178 , thus supporting the recent proposed model from the Ladurner group 175 .

The Pob3-MD exhibits a tandem PH-domain and was shown to genetically interact in yeast with the single-strand DNA binding protein RPA (Replication Protein A) physiologically linking Pob3-MD to the replication fork and DNA repair ${ }^{142}$. A mutation screen for HU-sensitive but viable yPob3 mutants revealed for all strains either a Q308R or Q308K mutation. The Q308K mutation resulted in a Spt ${ }^{-}$phenotype indicating additional deficiencies during transcription.

Spt16-CTD/Pob3-CTD: The Spt16-CTD is highly acidic and conserved ${ }^{155}$. For example the last terminal patch of 76 amino acids in yeast contains up to $47 \%$ acidic residues such as aspartate and glutamate. They are thought to interact with the basic histones neutralizing the charge and might be involved in unwrapping DNA or stabilizing the "open state" of nucleosomes as described before ${ }^{101}$. Several C-terminal deletions in yeast are lethal ${ }^{180}$. Moreover, functional analysis and kinetic data were analyzed using a C-terminal deletion mutant which is based on the hSpt16 $\Delta 836$ 
deletion 82181 . Therefore, the recent data from Hondele et al. $\frac{175}{}$, indicates that effects observed previously using the hSpt16 $\Delta 836$ were due to the deletion of the U-turn motif and partially deletion of the second PH-domain of Spt16MD. Furthermore, Hondele and coworkers performed quantitative ITC measurements addressing this issue and revealed in addition to their observed hydrophobic Spt16-H2A-H2B-interaction an electrostatic Spt16-CTD interaction.

The acidic Pob3-CTD is conserved and contains in the last terminal patch of 75 amino acids in yeast, 14 times aspartate and 14 times glutamate but is also rich in serine. A deletion of the terminal 95 amino acids $\Delta Q 458$ was shown to be detrimental in yeast ${ }^{182}$. However, investigation of this region was not a topic of extensive research so far.

yNhp6 and HMG-box of SSRP1: Many HMG-box proteins are involved in chromatin remodeling 183 . HMG-box proteins are DNA binding proteins and several crystal or NMR structures are available. In yeast two isoforms, yNhp6a and yNhp6b are known. The yNhp6a protein is a non-specific DNA binding protein, leaves DNA underwound and bends the DNA in the Nhp6-bound state $\mathrm{155}^{1 / 184 \mid 185}$. Although Nhp6a is only loosely associated with the yFACT complex and a deletion of Nhp6 is not detrimental, it assist yFACT function in vivo 159]186/187. Moreover, for structural reorganization of nucleosomes in an in vitro assays, an approximately 10 -fold molar excess of yNhp6 over Spt16-Pob3 heterodimer is needed ${ }^{188}$. In addition to its role for the FACT complex recruitment, several chromatin regulators such as Swi/Snf, RSC, Spt elongation factors, and Paf-1 show synthetic defects in combination with a Nhp6 deletion 185 . Therefore, Nhp6 might play a general role for many different remodeling processes in yeast.

\subsubsection{Mechanistic insights into FACT activity}

In 2011 Duane Winkler and Karolin Luger performed excessive binding studies using a fluorescent assay determining numerous thermodynamic parameters of hFACT and hFACT mutant interactions to several different nucleosome types and histone particles 181 . FACT shows a 20-fold binding preference for $\mathrm{H} 2 \mathrm{~A}-\mathrm{H} 2 \mathrm{~B}$ over $\mathrm{H} 3-\mathrm{H} 4$ in vitro. The lack of histone tails decreases the binding affinity 7-fold showing the importance of the histone tails for FACT activity and furthermore concluding that several multiple binding events between histones and the chaperone are necessary. Although Spt16 and SSRP1 alone are able to bind to nucleosomes, the tightest binding is observed for the complex of both. Hence revealing synergistic binding events for maximum functionality. Furthermore, in a direct competition assay, they showed that 
FACT can remove H2A-H2B from DNA but not $\mathrm{H} 3-\mathrm{H} 4$. Thus FACT can outcompete DNA for H2A-H2B binding and therefore reduce non-nucleosomal histone-DNA interactions. SSRP1 binding is increased when nucleosomes contained accessible linker DNA as compared to nucleosomes lacking this linker DNA. Moreover, they determined the stoichiometry of FACT within tri-nucleosome arrays and revealed that constructs containing the DNA-linker allow binding of FACT with a 3.3:1 ratio. Hence, FACT can bind at adjacent nucleosomes without sterical hinderance. In conclusion, Winkler and Luger proposing a mechanism in which first Spt16 is involved in tethering to nucleosomes and intercalating between DNA and octamer. Second, the unwrapped DNA can be fetched by SSRP1 containing the HMG-box domain.

\subsubsection{The dimer eviction and global accessibility model}

In general there are two mechanistic models describing FACT complex activity for nucleosome reorganization: the dimer eviction and the global accessibility model (figure 1.10) 153155. The dimer eviction model was deduced from the initial experiments revealing $\mathrm{H} 2 \mathrm{~A}-\mathrm{H} 2 \mathrm{~B}$ dimer displacement leading to hexasome formation during RNA-polymerase translocation through chromatin templates in vitro ${ }^{82}$. This model claims, that FACT function is acting by destabilizing the dimer-tetramer interactions leading to the eviction of a $\mathrm{H} 2 \mathrm{~A}-\mathrm{H} 2 \mathrm{~B}$ dimer and a more accessible DNA 156 . Initial experiments with crosslinked octamers, which abolished FACT-dependent transcription in vitro, support this model 161 . Furthermore, FACT-dependent H2A-H2B dimer loss is observed in vivo by ChIP experiments at the PHO5 promoter in yeast during transcriptional induction 150 .

The second proposed model is mainly supported by a recent study from Xin et al., published in 2009 189 . They performed restriction endonuclease and hydroxyl radical experiments on reconstituted yeast nucleosomes in the presence or absence of yFACT or Nhp6. They show extensive DNA accessibility in the presence of FACT without displacement of the H2A-H2B dimer. They observed increased accessibility by nuclease cleavage at sites distributed across the nucleosomal DNA. Using ChIP and quantitative PCR, they measured the occupancy of $\mathrm{H} 3$ and $\mathrm{H} 2 \mathrm{~A}$ at the inducible GAL1-10 promoter and observed rapid nucleosome eviction as early as 10 minutes. However, the ratio between $\mathrm{H} 3$ and $\mathrm{H} 2 \mathrm{~A}$ does not change over time as someone would expect if the dimer eviction model would preferentially apply. In summary, they suggest a "global accessibility model" where yFACT induces reorganization of the nucleosome whereas Nhp6 is implicated in an initial reorganization prior to Spt16-Pob3 action. In addition, the loss of the H2A-H2B dimer would be an indirect consequence but not the primary aim of the FACT complex action. They speculate 
that the nucleosomal components stay tethered so that reformation of the canonical form can easily occur. This model would explain how a tight equilibrium between the canonical form and the reorganized form can mediate several functions of FACT 189 . However, some parts of the model are specific for the yeast or the human FACT complex, mainly due to excessive amount of Nhp6 needed for a functional yFACT complex 188 . Therefore, a combinatorial model or different models for different cellular tasks during transcription, replication or DNA repair are possible ${ }^{155}$.

\subsubsection{FACT during transcription, replication and DNA repair}

\subsubsection{Roles during transcription initiation and elongation}

FACT is shown in several studies to be involved in transcription initiation. The yeast and human FACT complex interact physically with the general transcription factor TFIIE/yTFIIE ${ }^{190}$. A promoter specific role in transcription initiation is shown by the requirement of FACT for TBP recruitment to GAL promoter ${ }^{166 \mid 191}$. FACT promotes TBP and TFIIA binding to nucleosomal TATA-sites ${ }^{164}$. Furthermore, FACT acts in concert with Asf1 evicting nucleosomes at the URS2 element of the HO promoter in vivo 192 .

A key study from Mason et al. in 2003, revealed that FACT travels along with the RNA-polymerase II through coding regions $166[193$. Genome-wide profiling of the RNA-polymerse II as presented by Mayer et al., shows that Spt16 and Paf1 are a part of the elongation complex working upstream of the polyadenylation site ${ }^{194}$. FACT is thought to be indirectly associated to RNA-polmerase II through binding to the Paf1 complex ${ }^{195 / 196 / 197}$. Futhermore, the H2BK120 monoubiquitination and Paf1 stimulate FACT-dependent transcription on chromatin templates in vitro 198 . However, a monoubiquitination on lysine 119 of H2A shows an opposite effect and is involved in transcriptional repression 199 . It blocks recruitment of FACT to the promoter and prevents RNA-polymerase II release. In addition to the recruitment function of Paf1, a recent study from Kwon and colleagues ${ }^{200}$, reveals that HP1 can guide and link FACT to active polymerase.

FACT is known to interact with several elongation factors 196/201. The ATP-dependent remodeler Chd1 interacts and colocalizes with SSRP1 in Drosophila ${ }^{202}$. The human Chd1 can interact to $\mathrm{H} 3 \mathrm{~K} 4 \mathrm{me} 3^{203}$, a classical mark for active chromatin and therefore might bridge FACT to actively transcribed genes 204 . However, the specific interaction of Chd1 to methylated H3K4 is not conserved in yeast ${ }^{204}$. In addition to the association of FACT with RNA-polymerase II, a recent study from Birch et al., reveals that hFACT copurifies with mammalian RNA-polymerase I and associates with RNA 
Polymerase III complexes ${ }^{205}$. Thus FACT is also implicated in facilitating chromatin transcription at rRNA and tRNA genes. The FACT complex is additionally thought to function during reassembly of nucleosomes during transcription since several yFACT mutations show increased cryptic promoter expression $165[166|206| 207$.

The chromatin structure, especially the nucleosomes, are an obstacle for the translocating RNA-polymerase ${ }^{208}$. Despite the above described model of dimer eviction causing hexasome formation $\underline{82.155}$, a more recent collaborative publication from Karolin Luger, Danny Reinberg and Vasily M. Studitsky proposes a different mechanism for hFACT deduced from excessive in vitro data. They claim that RNApolymerase II can travel through a nucleosome template mediated by hFACT without dimer displacement or reloading after the passage of the polymerase ${ }^{209}$. Their data suggests that hFACT competes with DNA through alternating interactions with the H2A-H2B dimers. Furthermore, they show that histone crosslinking within the nucleosome does not affect the function of hFACT during transcription, thus concluding that FACT mediates its function by $\mathrm{H} 2 \mathrm{~A}-\mathrm{H} 2 \mathrm{~B}$ dimers in their intranucleosomal locations. The translocation of the RNA-polymerase through the nucleosome template is assisted by the proposed $\Phi$-loop model 209|210|211. The authors claim that first FACT may destabilize the nucleosome and second DNA is partially uncoiled from the nucleosome as the polymerase enters the nucleosome. As the polymerase proceeds, the DNA behind is recoiled and the $\Phi$-loop is formed, which induces more uncoiling in front of the polymerase complex. Thus allowing polymerase progression through the nucleosome. The complex interplay of hFACT interacting with the proximal or distal H2A-H2B dimer in an alternating fashion during this progression process is crucial for the nucleosome survival.

\subsubsection{Functions of FACT in replication}

FACT is physically and genetically connected to various factors of the DNA replication machinery such as DNA-polymerase $\alpha^{158 \mid 212[213}$, the replicative MCM helicase complexes ${ }^{143}$ and the single-strand DNA binding protein RPA ${ }^{142}$. Early studies of T160, the murine homologue of SSRP1, revealed colocalization with DNA replication foci by indirect immunofluorescence staining in mouse fibroblasts 214 . The authors show that T160 is down-regulated during cell differentiation from mouse myoblasts to differentiated myotubes. This is consistent with a role of T160, respectively FACT, during replication since replication fades out during differentiation ${ }^{215}$. In Xenopus laevis eggs extracts, FACT function is crucial for maintaining normal levels of DNA replication $170[216$.

Sensitivity to hydroxyurea (HU), where HU decreases the dNTP pool and inter- 
feres therefore with DNA replication ${ }^{217}$, is a typically observed phenotype used by yeast geneticists to monitor replication defects. In 2000 Schlesinger and Formosa ${ }^{182}$ performed a Pob3 mutagenesis screen in yeast and revealed several pob3 mutants possessing HU-sensitivity and transcription defects indicated by the Spt ${ }^{-}$phenotype $\mathrm{e}^{218 / 219 \mid 220}$. The authors report genetic interactions to several replication factors such as CTF4 (Chromosome Transmission Fidelity), DNA2 (DNA synthesis defective), CHL12 (Chromosome Transmission Fidelity) and to POL1 (DNA-polymerase $\alpha$ ). Combining defects in the S-phase checkpoint gene MEC1 with pob3 mutations decreased cell survival up to 100-fold as compared to single mutants, thus, concluding a strong connection of Pob3 and yFACT to replication.

A study from Tan and coworkers revealed several aspects of FACT and its physical and functional interactions to MCM helicase complex ${ }^{143}$ : FACT interacts with two subcomplexes of MCM, MCM2-7 and MCM4/6/7 which was confirmed by immunoprecipitation experiments. The FACT complex coexists with MCM on replication origins in vivo. Furthermore, FACT promotes the DNA helicase activity of MCM4/6/7 on nucleosomal templates in vitro.

A recent paper from Kundu and colleagues used a Xenopus egg extract cell-free system to analyse the binding dynamics of FACT during replication 221 . The authors revealed a biphasic process consisting of an initial binding phase which can be removed and a second binding of FACT after origin licensing whereas the later is crucial for efficient replication.

\subsubsection{The role of FACT in DNA repair}

There are two phenomena described for the FACT complex in DNA repair. First, the histone variant H2A.X is phosphorylated at serine 139 upon $\gamma$-irradiation representing the classical histone mark for double strand breaks ${ }^{40[222}$ (see section 1.2.2.2). FACT is shown to mediate the exchange of H2A.X which is stimulated upon H2A.X phosphorylation 223 . However PARP1-mediated poly-ADP-ribosylation of Spt16, which is increased upon genotoxic stress 224 , inhibits H2A.X-H2B dimer exchange. In addition, poly-ADP-ribosylation of Spt16 is shown to inhibit nucleosome binding in general in vitro ${ }^{224}$. In conclusion, poly-ADP-ribosylation might be a mechanism to maintain H2A.X on chromatin, whereas after DNA repair and absence of the ribosylation signal, FACT could mediate the $\gamma$-H2A.X removal $223 / 225$.

A second observation in response to UV-mediated DNA damage was reported by Keller and colleagues 171226 . They show that FACT forms a complex with CK2 (casein kinase II) which in turn phosphorylates serine 392 of p53227. A successive in vitro study suggests a specificity change of the CK2 induced by FACT 171 . The 
S392 phosphorylation is important for functionality of p53228/229 - a key player in DNA damage and cancer 230 . Interestingly, CK2 additionally phosphorylates SSRP1 on serines 510, 657 and 688 in vitro which important for the regulation of the DNA binding ability of SSRP1231. SSRP1 phosphorylation upon UV-irradiation was confirmed in vivo. A more recent study from 2009, reveals the native phosphorylation state of Drosophila melanogaster SSRP1 expressed in insect cells 173 . They found several phosphorylated sites in the intrinsically disordered SSRP1-ID region which controlled nucleosome binding. Their final model claims a higher degree of DNA-binding regulation due to an inhibition mechanism by phosphorylation of the acidic ID region which interacts in the phosphorylated state with the HMG-domain blocking dsDNA binding. Furthermore, they suggest an storing function of SSRP1 upon high phosphorylation which was observed in the fly ovary but lost in the developing embryo. They conclude that dephosphorylation activates FACT supporting rapid DNA replication and transcription of the developing embryo.

\subsection{Genetic code expansion - a versatile tool for genetically encoding unnatural amino acids}

Genetic code expansion is broadly defined as a genetic toolkit for the incorporation of unnatural amino acids into proteins. The incorporated non-canonical amino acids can harbor different chemical modifications which give rise to "designer" proteins with newly defined properties ${ }^{72 \mid 232}$. There are roughly one hundred different unnatural amino acids to date. These synthetic amino acids contain altered sidechain moieties including preinstalled post-translational modifications, chemically reactive handles, photocage protection groups or photoactive crosslinkers. These probes were successfully incorporated into recombinantly expressed protein as well as introduced for in vivo studies in several organisms. The Genetic code expansion community is steadily increasing and scientists are further optimising and improving this toolbox $233|234| 235 \mid 236$.

\subsubsection{The Genetic code expansion principle}

The use of non-canonical amino acid analogues in various organisms can be dated back to the $1940 \mathrm{~s}^{237}$. One of the key experiments was performed by Cowie and Cohen in $1957^{238}$. They demonstrated the global exchange of methionine with selenomethionine in auxotrophic bacteria. Thus showing that bacteria can grow although an entire amino acid is substituted. The site specific-incorporation of a 
chemically modified amino acid using the Genetic code expansion technique was established decades later.

With a few exceptions ${ }^{239 \mid 240}$, the genetic code is a degenerated nucleobase triplet code encoding the 20 canonical amino acids with 61 different nucleobase combinations. Three codons are generally designated for the termination of translation, the stop codons amber (UAG), opal (UGA) and ochre (UAA). Molecularly, the genetic code is established by the transfer RNA (tRNA) containing the nucleobase combination in the anticodon loop. The tRNA is charged with its appropriate amino acid by the protein tRNA synthetase and used in a "key-lock" principle at the ribosome during translation. Genetic code expansion uses this nexus incorporating unnatural amino acids through the redefinition of one codon, preferentially the UAG amber codon. In practice, an additional tRNA $\mathrm{CUA}_{\mathrm{A}}$ along with its cognate aminoacyl tRNA synthetase (tRNA synthetase(AARS)/tRNA pair) need to be introduced to the system. The pair must be orthogonal and not recognized by the endogenous machinery. It must allow for the incorporation of the specific amino acid in response to the amber stop codon and not a natural amino acid (general principle applied for the incorporation of photocrosslinker amino acids is depicted in figure 1.11 and section 1.5.3.3).

Such a system was demonstrated for the first time by Furter in $1998^{241}$. He incorporated $p$-fluoro-phenylalanine into DHFR (dihydrofolate reductase) for subsequent NMR studies using a yeast PheRS/tRNAPhe CUA pair in Escherichia coli. However, major progress for the site-specific incorporation was achieved by the Schultz lab in 2001 242. Wang and colleagues changed the AARS amino acid specificity of the Methanocaldococcus jannaschii TyrRS/tRNA tyrosine by in vitro evolution of the TyrRS through several rounds of selection. They randomized five residues in the active site of the TyrRS suspected to be involved in recognition of the tyrosine moiety, deduced from a homologue crystal structure of Bacillus stearothermophilus. The PCR-based in vitro evolution and selection approach for functional evolved AARS is the cornerstone of genetic code expansion giving rise to the plethora of unnatural amino acids which can be incorporated. Using this evolved pair, they could express DHFR with a single $O$-methyl-L-tyrosine at the third amino acid with acceptable yields in E. coli.

Limitations of the system typically arise from sterical and chemical properties of the accepted amino acids as well as the incorporation efficiency which critically depends on the uptake of the UAA into the system 232 . Another common system for the incorporation of lysine derivatives was adapted and evolved from the pyrrolysine incorporation system in response to the amber codon from the archaea Methanosarcina barkeri. Despite the use of the GCE system for expression in Escherichia coli, unnatural 
amino acids have been successfully incorporated in eukaryotic cells as well as in several organisms such as flys and worms in vivo (for a broad review ${ }^{243}$ ). The sitespecificity of this approach makes Genetic code expansion tremendously appealing. Some selected examples and discoveries are discussed in the next section.

\subsubsection{Recent applications of the Genetic code expansion system}

Post-translational variants of lysines such as actelyated lysine can be incorporated into protein using an evolved pyrrolysine Genetic code expansion system in E. coli244. Employing FRET analysis on reconstituted nucleosomes that contained recombinantly expressed and isolated H3K56ac histones, Neumann and colleagues could study and gain deep insights into DNA breathing and the implications of this specific acetylation on nucleosome dynamics ${ }^{75}$. Attempts for the incorporation of methylated lysine were made and mono- as well as di-methylated lysine could be successfully incorporated using additional protection chemistry 245/246. Another evolving branch is represented by the incorporation of chemical handles ,for example cyclooctynes and azides, which can react in a copper-free environment under the formation of covalent structures called "Click-Chemistry"247. Using the modified pylRS/tRNA AF pair, Plass and coworkers incorporated lysine derivatives containing a cyclooctyne moiety in GFP and used this for subsequent labeling of the purified protein with an azide derivative containing Atto647N. Click chemistry approaches were further improved in the lab of Jason Chin and raised to a new level. Lang and colleagues demonstrated in vivo labeling on the surface and even in mammalian cells using genetically encoded trans-Cyclooctenes or norbornene in subsequent reactions with tetrazine-fluorophore dyes 248249 . A third branch of the Genetic code expansion field involves the incorporation of photo-labile amino acids. These include photo-caged amino acids and nucleobases which can be deprotected, and thus activated by light. Coupling the activity of an enzyme or the accessibility of a DNA sequence with light revealed interesting cellular applications such as light-activated gene expression in mammalian cells ${ }^{250}$ or zebrafish 251 and in vivo riboswitch control in Escherichia coli 252 . Other important photo-activatable amino acids are the UV-inducible crosslinker amino acids for in vitro and in vivo assays, which are discussed in detail in the next section. 


\subsubsection{Using genetic code expansion for the incorporation of light-inducible crosslinkers into proteins}

\subsubsection{The photo-crosslinkers: 4-Benzoyl-L-phenylalanine (pBPA) and 4-Azido-L-phenylalanine (AzF)}

UV-inducible crosslinkers are versatile tools for studying protein-protein and proteinnucleic acid interactions in living organisms 232 253. The common functional groups of photo-crosslinkers are benzophenone, aryl azide, trifluoromethylphenyl diazirine and alkyl diazirine. They differ in properties such as activation wavelength, crosslinking efficiency and chemical characteristics such as hydrophobicity and charge. In this regard, thoughtful considerations need to be done before performing experiments. In my work, I used the benzophenone (4-Benzoyl-L-phenylalanine, pBPA) and an aryl azide (4-Azido-L-phenylalanine, pAzF).

Benzophenone and its crosslinking mechanism has been extensively described by Dormán and Prestwich in $1994^{254}$. Activation is performed by UV-light in a range from 350-365 nm. This leads to the formation of a diradical which can form covalent crosslink adducts by hydrogen abstraction from a neighboring $\mathrm{C}-\mathrm{H}$ bond (chemical structure of pBPA and the diradical form in figure 1.11A. top). Proton extraction can occur in a distance of $3.1 \AA$ from the center of the ketone oxygen. The excited state persists for 80-120 $\mu$ s until relaxation to the ground state occurs if not surrounded by a suitable interaction partner. Moreover, pBPA can be excited again possessing reversible excitability. Its reversible nature is one of the advantages compared to other crosslinkers such as the aryl azides. Furthermore, a certain preference of BPA for methionine, referred as the "methionine magnet", was demonstrated by Wittelsberger and colleagues in 2006 256 . The second crosslinker used in this studies contains an aryl azide function: 4-Azido-L-phenylalanine. After activation with UV-light of a broad range from 254-400 $\mathrm{nm}$, nitrogen gas is released and the formed nitrene can insert at $\mathrm{C}-\mathrm{H}$ bonds and heteroatom-H bonds forming covalent crosslinks (see figure 1.11A. bottom). In comparison to $\mathrm{pBPA}, \mathrm{pAzF}$ is less hydrophobic and significantly smaller and therefore less bulky. As described above, the nitrene formation is irreversible. Additionally, the highly reactive species lasts for approximately $100 \mu \mathrm{s}$ and is subsequently converted by ring expansion to the less reactive ketenimine which only reacts with nucleophiles $257 / 258$. Furthermore, the reduction of the azide to an amine $^{259}$ and therefore the loss of ability to form reactive species might influence the applicability of aryl azides in biological systems. 
A.<smiles>NC(Cc1ccc(C(=O)c2ccccc2)cc1)C(=O)O</smiles>

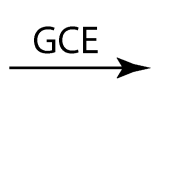

4-Benzoyl-L-phenylalanine (pBPA)<smiles>[N-]=[N+]=Nc1ccc(C[C@H](N)C(=O)[O-])cc1</smiles>
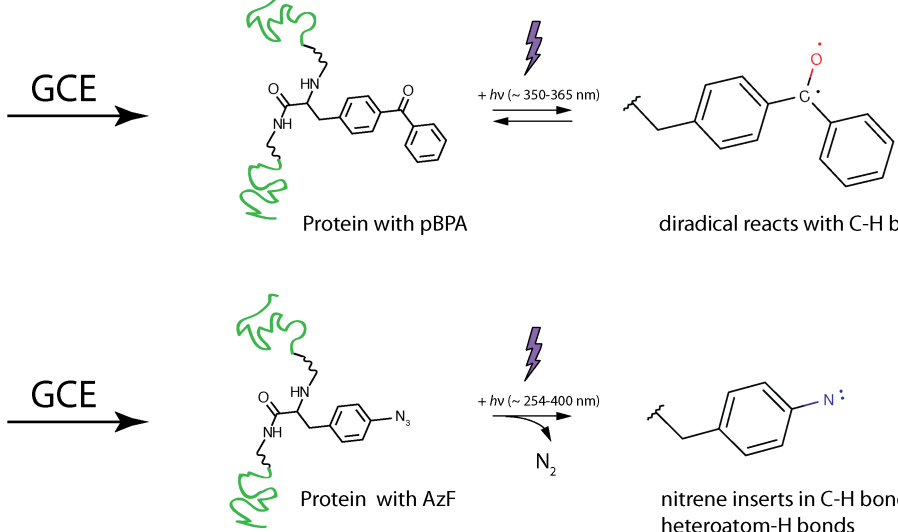

diradical reacts with $\mathrm{C}-\mathrm{H}$ bonds
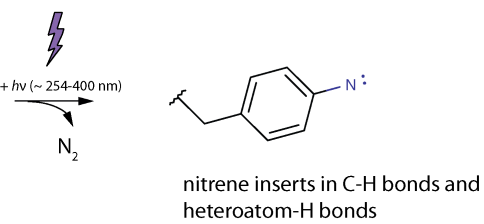

4-Azido-L-phenylalanine (AzF)

B.
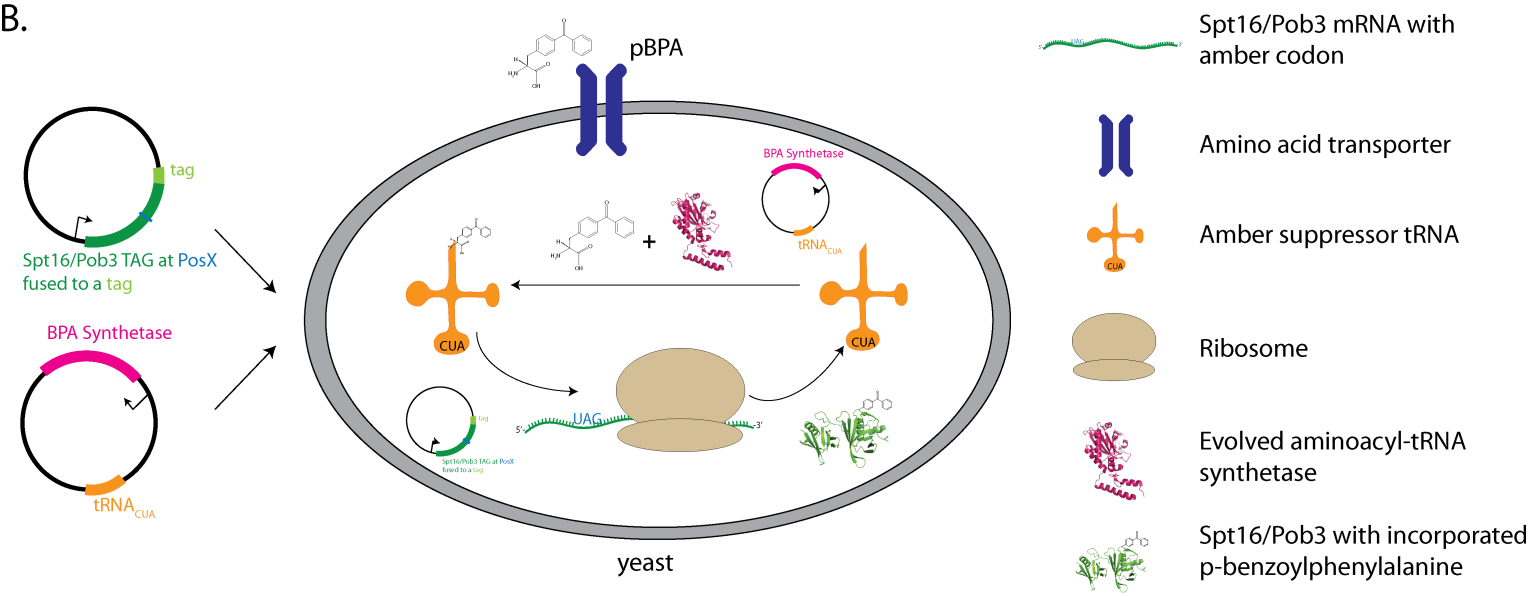

Figure 1.11: Genetically encoded photo-crosslinkers in Saccharomyces cerevisiae - A. Chemical structures of the crosslinker amino acids 4-Benzoyl-L-phenylalanine (pBPA) and 4-AzidoL-phenylalanine (pAzF). After the incorporation into proteins, irradiation with UV light induces radical formation which can form covalent crosslink adducts. Depicted is the highly reactive photogenerated species (for detailed chemistry see 253254 ). Activation of pBPA is reversible whereas nitrene formation is not. B. Genetic code expansion approach for the incorporation of crosslinker amino acids into Spt16/Pob3 in response to the amber stop codon in yeast. An evolved tRNA synthetase/tRNA pair allows incoportation of pBPA at designated amber sites of the Spt16 or Pob3 protein during translation in vivo (see text for details) ${ }^{255}$. The scheme can be adapted for the incorporation of pAzF using the evolved synthetase specific for this particular amino acid. 


\subsubsection{Incorporation of UV inducible crosslinker amino acids in Escherichia coli}

A genetic code expansion system for the incorporation of UV-inducible crosslinkers was established in several organisms. In this thesis, I used the system for expression and subsequent purification of pBPA-containing proteins from Escherichia coli $233 / 260$ and the system for in vivo crosslinking in yeast ${ }^{255}$. The E.coli system was developed by Chin and colleagues. They used the Methanocaldococcus jannaschii TyrRS/tRNACUA pair and changed the amino acid specificity to pBPA by in vitro evolution with five rounds of positive and negative selection. After isolating a functional BpaRS/tRNA pair, they performed proof of principle experiments expressing myoglobin and GST containing pBPA. For GST, they incorporated pBPA at two different positions either at the GST-dimer interface (F52) or on opposite sites of the dimer (Y198). Subsequent crosslinking of the purified proteins and SDS-PAGE analysis showed UV-dependent covalent dimerization only at position F52. Thus, beautifully demonstrating that the site specific incorporation and crosslink analysis can be used to prove protein-protein interactions. A similar TyrRS/tRNA pair was evolved for the incorporation of $\mathrm{pAzF}$ in a similar approach and experimental setup 261. This efficiency of the system was further improved by Ryu et al. developing a single plasmid system, exchanging the AARS promoter and modifying the copy number and sequence environment of the suppressor tRNA 233 . Three selected studies using the E.coli system for pBPA incorporation are discussed in the next paragraph.

In vivo pBPA crosslinking was performed by Mori and coworkers studying proteinprotein interactions and mapping the interaction surface between SecY and SecA, two components involved in protein translocation across the plasma membrane in E.coli262. They incorporated pBPA at several different positions of SecY and were able to map interaction surfaces to SecA and further gained mechanistic insights by in vitro pBPA crosslink experiments. Furthermore, a reciprocal study introducing the crosslinker in SecA was described by Das and Oliver in 2011263. Another study examined the bacterial chaperone $\mathrm{ClpB}^{264}$. The authors identified a crucial substratebinding site at the central pore of the chaperone. In 2008, Merz and colleagues analyzed the TF (trigger factor) which is bound to the ribosome and promotes chaperoning of the nascent polypeptide chains. They were able to crosslink from the chaperone to the nascent arrested peptide chain dissecting the sequence of binding events of chaperone-assisted protein folding during translation 265 .

Besides analyzing protein-protein interactions, a basic study performed by Lee and colleagues used pBPA crosslinking to study protein-DNA interactions 266 . They incorporated pBPA into Escherichia coli catabolite activator protein (CAP) and performed 
in vitro crosslinking studies with the dsDNA fragment containing the CAP binding site. They could show specific crosslinking to the DNA fragment and confirmed that the pBPA moiety itself did not influence the binding to dsDNA, thus corroborating specificity and applicability of pBPA for protein-DNA crosslinking studies.

\subsubsection{Incorporation of pBPA and pAzF in and Saccharomyces cerevisiae and higher eukaryotes for UV-inducible crosslinking}

The genetic code for Saccharomyces cerevisiae was expanded by Chin and colleagues in 2003 255 . Instead of using a TyrRS/tRNA pair from archea, they used the TyrRS/ tRNATyr ${ }_{\text {CUA }}$ pair from Escherichia coli which was suspected to be orthogonal in yeast since the translation machinery between prokaryotes and eukaryotes is less conserved. Interestingly, a natural isolated amber suppressor tRNA from Escherichia coli suppressor mutants was already described in 1968 from Goodman et al.267. The eukaryotic in vitro evolution by Chin and coworkers was similarly done as compared to the prokaryotic system by randomizing the implicated five amino acids of the AARS. Since selection was performed in Saccharomyces cerevisiae, they adapted the selection procedures from using bacterial antibiotic resistance gene to the use of auxotrophy markers in yeast. Selection was performed using the HIS and URA auxotrophy markers as well as a lacZ gene for colorometric assay, thereby achieving TyrRS/tRNA pairs for the incorporation of several amino acids such as pBPA (pbenzoylPheRS-2) and pAzF ( $\mathrm{p}$-azidoPheRS-3) 255 . These were extensively used in my thesis. During the last decade, this system was used for several in vivo crosslinking studies in yeast which are discussed in the following section.

\subsubsection{Applications of pBPA-crosslinking in yeast}

Benzophenone crosslinking has been used in studies to address membrane transport processes such as in the analysis of the ERAD-pathway (Endoplasmic-reticulumassociated protein degradation) ${ }^{268 / 269}$ or the mitochondrial import $270 \mid 271$. Tamura and coworkers used in vivo crosslinking in yeast to elucidate the network between the outer- and inner membrane complexes, especially the role of the intermembrane space domains of Tim 23 and Tim50, and their role for the mitochondrial import machinery 270 . Another recent study used this approach to prove the mutually exclusive interaction between Tom 7 and two $\beta$-barrel proteins in the outer membrane, Tom 40 and $\mathrm{Mdm} 10^{272}$. In addition to the in vivo pBPA studies, a recent pBPA crosslinking study used isolated mitochondrial systems and pBPA-containing peptides to analyze signal-binding sites of presequence receptors during mitochondrial import 271 .

Pedro Carvalho and coworkers in Tom Rapoports lab performed crosslinking 
experiments analyzing the ERAD-L pathway, specialized for misfolded luminal ER proteins 268 . They used a HA-tagged ERAD-L substrate and incorporated pBPA at various different positions. By HA-immunoprecipitation and western blot analysis, they could map the interactions of the substrate with several components of the ERAD-L pathway in a site specific manner, thus elucidating Hrd1p as the key component. Furthermore they studied the effect of glycosylation of ERAD-L substrate which appears to be important for the interaction of Hrd1p in vivo. A successive study used the similar approach analysing Hrd3p, another component of the ERAD-L pathway Hrd1 complex 269 .

In addition to the analysis of a small amount of different crosslinking positions a more comprehensive study was published by Mohibullah and Hahn in 2008273. They incorporated pBPA at 61 different surface-exposed positions of the TATA-binding protein (TBP) in Saccharomyces cerevisiea and performed excessive in vivo crosslinking studies. They completely exchanged the endogenous TBP with pBPA-containing mutants using a yeast shuffle strain. This approach led to 24 viable mutant strains which were mainly used for nuclear extraction and reconstitution of preinitiation complexes (PICs) with subsequent crosslinking. However, only 10 position showed reproducible crosslinking patterns. Using molecular shift approaches in FLAGtagged yeast strains, they could identify and map several interaction partners such as the transcriptional repressor Mot1, or the SAGA complex subunits Spt8 and Spt3. The Mot1 interaction was verified by in vivo crosslinking of nuclear extracts prior to PIC assembly. Nevertheless, the main focus of this study was testing interactions of TBP with assembled PICs on the immobilized HIS4 promoter, thus performing a preselection.

All these examples reveal possibilities of analyzing structural and mechanistic details of various cellular processes in vitro and in vivo.

\subsubsection{Photoaffinity crosslinking in higher eukaryotes and for the analysis of DNA-protein interactions}

In addition to pBPA studies in yeast, Hino and coworkers have adapted the crosslinking system for mammalian cells ${ }^{274}$. For efficient incorporation they combined the evolved pBpaRS from E.coli255 in combination with a Bacillus stearothermophilus suppressor $\mathrm{tRNATyr}$ CUA. This specialised suppressor tRNA was already successfully used to incorporate 3-iodo-L-tyrosine into epidermal growth factor receptor (EGFR) or Ras in Chinese hamster ovary $(\mathrm{CHO})$ cells ${ }^{275}$. In the successive study from Hino and colleagues in 2005, pBPA was incorporated into the SH2 domain of Grb2, an adaptor protein of EGFR. Using this system in CHO cells, they could beautifully 
demonstrate site-specific EGFR-Grb2 interactions upon stimulation with EGF in vivo. In a successive improved approach published in 2011, they used the genetically encoded $p$-trifluoromethyl-diazirinyl-L-phenylalanine crosslinker and combined their approach with a SILAC masspectrometry analysis 276 . They could distinguish between direct binders of the certain GRB2-SH2 and noncovalently binders of the two Src homology 3 domains. In conclusion, especially the groups of Shigeyuki Yokoyama and Kensaku Sakamoto revealed a thrilling potential for the application of pBPA and other photo-affinity crosslinking methods in more complex systems. In addition to EGFR signaling, the analysis of GPCRs (G-protein-coupled receptors) was extensively studied using unnatural amino acids in mammalian cells 277278 .

\subsection{Aims}

This work combines current structural information of the histone chaperone complex FACT with an in vivo approach using genetically encoded UV-inducible crosslinker amino acids in Saccharomyces cerevisiae. My aim is to develop and characterize an in vivo crosslinking scan to map protein-protein interactions within the FACT complex and to interacting proteins such as histones. I want to perform the analysis in a high-throughput fashion, creating a FACT complex library of approximately 200 mutants each containing a single site-specific crosslinker. This library would allow me to scan in a comprehensive manner. I want to perform molecular shift assays in genomically-tagged yeast strains and further begin proteomic analysis to identify novel and known interaction partners of FACT in a site-specific way. The insights of this screen would allow me to focus on particular aspects of the FACT complex and provides a starting point for further investigations into dynamics of identified interactions to environmental challenges or the cell cycle. 



\section{Material and Methods}

\subsection{Material and equipment}

\subsubsection{Equipment, consumable supplies and chemicals}

\subsubsection{Technical apparatuses}

- ABI PRISM ${ }^{\circledR} 3100$ DNA Sequencer

• ÄKTApurifier 10 (\#28-4062-64) and ÄKTAprime/plus (\#11-0013-13), GE Healthcare / Amersham Pharmacia Biotech

- Allegra 2IR centrifuge, Beckman Coulter

- Avanti-J20 XPI centrifuge, Avanti-J30 centrifuge, JLA 8.1000 and JA-30.50 Ti rotor, Beckman Coulter

- BioPhotometer, Eppendorf

- Consort EV231 electrophoresis power supply (\#Z654353), Sigma, Consort

- Criterion ${ }^{\mathrm{TM}}$ Blotter with plate electrodes (\#170-4070), Bio-Rad

- Eppendorf ${ }^{\circledR}$-Thermomixer comfort ${ }^{\mathrm{TM}}$ (\#5355 000.011), Eppendorf

- Dounce tissue grinder set; $7 \mathrm{~mL}$ working volume; pestle B clearance 0.00080.0022 in. (\#D9063), SIGMA

- Gel Doc ${ }^{\text {TM }} 2000$ (\#170-8170), Bio-Rad

- Heating Cabinet / Incubator, Mytron Bio- und Solartechnik GmbH

- Incubation Shaker Multitron, INFORS HT

- Labcycler (\#011-103), SensoQuest

- Microcentrifuge Eppendorf ${ }^{\circledR}$-Centrifuge 5414 R (\#5426 000.018), Eppendorf

- Mini Trans-Blot ${ }^{\circledR}$ Electrophoretic Transfer Cell (\#170-3930), Bio-Rad

- NanoDrop ND-1000 Spectrophotometer, Thermo Scientific

- OPTIMAX ${ }^{\circledR}$ X-ray film Processor (\#1170-1-0000), Protec

- PerfectBlue ${ }^{\mathrm{TM}}$ SEDEC M 'Semi-Dry' Electroblotter (\#52-2020), PEQLAB

- Peristaltic pump drive PD 5001 (\#523-50010-00) with Pumphead C4 (\#524-80420-00), Heidolph

- QuantEM:512SC EMCCD Camera, Photometrics

· Rotamax 120 Shaker (\#544-41200-00), Heidolph 
- Sonifier 250 (\#101-063-197), Branson

- Spinning disk confocal unit (CSU-X1), Yokogawa

- Typhoon 9400 variable mode imager (\#63-0055-78), GE Healthcare / Amersham Biosciences

- Ultra Centrifugal Mill ZM 200, Retsch

- Vertical Electrophoresis System H10, Omnilab

- UV-lamp, Vilber Lourmat VL-208.BL, 365nm tubes, 2x8W, Vilber

- XCell SureLock ${ }^{\circledR}$ Mini-Cell and XCell II ${ }^{\mathrm{TM}}$ Blot Module (\#EI0002), Life Technologies

· Zeiss AxioObserver.Z1 Inverted Microscope, ZEISS Germany

\subsubsection{Laboratory utensils}

- Eppendorf Research ${ }^{\circledR}$ (adjustable) $2.5 \mu$ l pipette (3120 000.011), Eppendorf

- Eppendorf Multipette ${ }^{\circledR}$ plus (\#4981 000.019), Eppendorf

- Hamilton Syringe 1705N 50 $\mu$ l, Hamilton

- Research plus ${ }^{3}$ : Eppendorf Research ${ }^{\circledR}$ Plus 3-Pack (0,5 -10 $\mu 1,10-100 \mu 1,100-$ 1000 pl) (\#3120 000.909), Eppendorf

\subsubsection{Consumable supplies}

- $15 \mathrm{ml}(50 \mathrm{ml})$ Conical Tubes, BD Falcon ${ }^{\mathrm{TM}}$

- 96-well cell culture plates, BD Falcon ${ }^{\mathrm{TM}}$

- Amersham Hybond ${ }^{\mathrm{TM}}$ LFP 0.2 PVDF (\#10600022), GE Healthcare

- Amersham Hyperfilm ${ }^{\mathrm{TM}}$ ECL (\#28906837), GE Healthcare

- Amicon Ultra-15 Centrifugal Filter Units with different MWCOs, Millipore

- Corning ${ }^{\circledR} \operatorname{Costar}^{\circledR}$ Spin- $X^{\circledR}$ centrifuge tube filters cellulose acetate membrane, pore size $0.45 \mu \mathrm{m}$, CLS8163, Corning, Sigma

- Costar Microcentrifuge tube $1.7 \mathrm{ml}$ pre-lubricated (\#3207), SIGMA, Corning

- Cuvettes (\#67.742), SARSTEDT

- Dialysis Membrane MWCO 12.4 kDa (\#D9277), SIGMA

- Glass-ware: Erlenmeyer flask (100 mL, 300 mL, 1000 mL, 2000 mL), Borosilicate flasks (100 mL, $250 \mathrm{~mL}, 500 \mathrm{~mL}, 1000 \mathrm{~mL})$, pipettes $(20 \mathrm{~mL}, 10 \mathrm{~mL}, 5 \mathrm{~mL})$

- Immobilon ${ }^{\circledR}$-P PVDF Membrane (\#IPVH00010), Millipore

- Micro tube $1.5 \mathrm{ml}$ SAFETY CAP (\#72.690), SARSTEDT

- Multiply- $\mu$ Strip $^{\circledR} 0.2 \mathrm{ml}$ chain (\#72.985.002) with 8-Lid chain, flat (\#65.986.002), SARSTEDT

- PCR 0.2 ml SoftTubes, domed cap, Biozym

- Petri dishes for bacteriology 92/16 mm with cams (\#82.1473), SARSTEDT 
· Pipette-tips $10 \mu \mathrm{l}$ (\#70.1130), $200 \mu \mathrm{l}$ (70.760.002), $1000 \mu \mathrm{l}$ (70.762), SARSTEDT

- Sorenson $^{\text {TM }}$ low binding M $\mu$ ltiFit Pipette Tips 1-200 $\mu \mathrm{l}$ (\#Z719579), 0.5-10 $\mu \mathrm{l}$ (\#Z719544), SIGMA

- SafeSeal micro tube $2.0 \mathrm{ml}$ (\#72.695), SARSTEDT

- Slide-A-Lyzer ${ }^{\circledR}$ MINI Dialysis Units 7,000 MWCO (\#69560), Thermo Scientific

- Spectra/Por ${ }^{\circledR}$ Dialysis Membrane MWCO 6-8.000 (\#132665), Spectrum Laboratories Inc.

· TipOne ${ }^{\circledR}$ RPT $10 \mu \mathrm{l}$ (\#S1180-3810), $200 \mu \mathrm{l}$ (\#S1180-8810), $1000 \mu \mathrm{l}$ (\#S1182-1830), STARLAB

• Vivaspin 6 concentrator MWCO 10.000, 30.000 (\#VS0601, \#VS0621), Sartorius

\subsubsection{Chemicals}

- 2-Propanol p.A. (\#A0900,2500GL), AppliChem

· 4-Azido-L-phenylalanine [206.1 g/mol] (\#06162), Chem-Impex International, Inc.

• 4-Benzoyl-L-phenylalanine [269.3 g/mol] (\#05110), Chem-Impex International, Inc.

- Ampicillin sodium salt pure Ph. Eur. [371.39 g/mol], (\#A6352,0025), AppliChem

· dNTP Mix, $10 \mathrm{mM}$ each (\#R0192), Thermo Scientific

- Boric acid extra pure [61.83 g/mol] (\#11606), Riedel-de Haen

- BSA - Albumin bovine serum (\#A7906-100G), Sigma

- Difco ${ }^{\mathrm{TM}}$ Skim Milk (\#232100), BD

· di-Sodium Hydrogen Phosphate 2-Hydrate [177.99 g/mol] (\#T877.1), Roth

- Dithiothreitol [154.25 g/mol] (\#A1101,0025), BioChemica, AppliChem

· EDTA $\geq 99 \%$ p.a. [372.24 g/mol] (\#8043.2), Roth

- Ethanol absolute AnalaR NORMAPUR (\#20821.321), VWR

. Glycine $\geq 99 \%$ p.a. [75.07 g/mol] (\#3908.3), Roth

- Glycerol bidistilled 99.5 \% AnalaR NORMAPUR (\#24388.295), VWR

- Guanidine hydrochloride pure [95.53 g/mol] (\#A4014,1000), AppliChem

- Guanidine hydrochloride $\geq 99 \%$ [95.53 g/mol] (\#50940), Sigma, Fluka ${ }^{\circledR}$ Analytical

· Imidazole [68.08 g/mol] (\#A1073,1000), AppliChem

- Isopropyl- $\beta$-D-thiogalactopyranosid $\geq 99 \%$ [238.3 g/mol] (\#CN08.4), Roth

- Kanamycin sulfate [582.58 g/mol], BioChemica, AppliChem

- Leupeptin [475.59 g/mol] (\#L2884), Sigma

- O-Phenanthroline [234.68 g/mol] (\#77510), Sigma

- Protease Inhibitor Cocktail P9599 (\#P9599), SIGMA

- Pefablock ${ }^{\mathrm{TM}}$ [239.69 g/mol] (\#76307), Sigma 
- Pepstatin A [685.89 g/mol] (\#77170), Sigma

- peqGOLD Universal Agarose (\#35-1020), PEQLAB

- Potassium chloride p.A. [74.56 g/mol] (\#A3582,1000), AppliChem

- Rotiphorese ${ }^{\circledR}$ Gel 30 (37,5:1): 30 \% Acrylamide/Bisacrylamide (\#3029.1), Roth

- Rotiphorese ${ }^{\circledR}$ Gel 40 (29:1): 40 \% Acrylamide/Bisacrylamide (\#A515.1), Roth

- SDS ultra pure $\geq 99.9 \%$ [288.38 g/mol] (\#2326.2), Roth

. Sodium chloride p.a. [58.44 g/mol] (\#A3597,5000), AppliChem

. Sodium dihydrogen phosphate dihydrate [156.01 g/mol] (\#A2944,1000), AppliChem

- Spectinomycin dihydrochloride pentahydrate [495.30 g/mol], (\#A3834,0005), AppliChem

- Tris Pufferan ${ }^{\circledR} \geq 99.9 \%$ [121.14 g/mol] (\#4855.3), Roth

- Triton X-100 [624 g/mol] (\#3051.2), Roth

- Tween ${ }^{\circledR} 20$ [1227.72 g/mol] (\#8.22184.0500), Merck

- Urea for biochemistry [60.06 g/mol] (\#1.08488.9010), Merck

- Yeast Nitrogen Base without Amino Acids \& Ammonium Sulfate (\#233520), Becton, Dickinson and Company

All the other common chemicals were purchased from Merck, Sigma or Roth

\subsubsection{Frequently used solutions and buffers}

Unless noted otherwise, water $\left(\mathrm{ddH}_{2} \mathrm{O}\right)$ was used for buffer preparations and was purified by the water-processing unit "Ultra Clear Plus" (Fahrenberg). In this thesis, media preparation was done using ion-exchange-purified water $\left(\mathrm{dH}_{2} \mathrm{O}\right)$. Solutions for long-term storage were filtered sterile.

\subsubsection{Solutions}

- LiOAc: $1 \mathrm{M} \mathrm{LiOAC}$

- 10x TE: $100 \mathrm{mM}$ TrisHCl pH 7.5, $10 \mathrm{mM}$ EDTA

. 50 \% (w/v) PEG 3350: $50 \mathrm{~g} / 100 \mathrm{~mL}$

- 1000x PIC: 75 mM Pefablock, $150 \mu$ M Leupeptin, 37.5 mM O-Phenanthroline, $500 \mu \mathrm{M}$ PepstatinA in DMSO

- pAzF-stock solution: $500 \mathrm{mM}$ 4-Azido-L-phenylalanine in $1 \mathrm{M} \mathrm{NaOH}$; store at $-20^{\circ} \mathrm{C}$

- Bio-Rad Protein Assay (\#500-0006), Bio-Rad

- pBPA-stock solution: $100 \mathrm{mM}$ 4-Benzoyl-L-phenylalanine in $120 \mathrm{mM} \mathrm{NaOH}$, filter sterile; store at $-20^{\circ} \mathrm{C}$

. GelRed: GelRed Nucleic Acid Stain (10.000x) in water (\#41003-0.5mL), Biotium 
- IPTG: $1 \mathrm{M}$ IPTG in $\mathrm{H}_{2} \mathrm{O}$

- InstantBlue ${ }^{\mathrm{TM}}$ : ready-to-use solution (\#ISB01L), Expedeon Protein Solutions

- Y-PER Yeast Protein Extraction Reagent: ready-to-use solution (\#78990), Thermo Scientific

\subsubsection{Media and supplements}

For the preparation of solid culture media plates $15 \mathrm{~g} / \mathrm{L}$ Agar was added prior to sterilisation.

- 2YT-media: $5 \mathrm{~g} / \mathrm{L} \mathrm{NaCl}, 10 \mathrm{~g} / \mathrm{L}$ Yeast Extract, $16 \mathrm{~g} / \mathrm{L}$ Bactotryptone

- LB-media: $5 \mathrm{~g} / \mathrm{L} \mathrm{NaCl}, 5 \mathrm{~g} / \mathrm{L}$ Yeast Extract, $10 \mathrm{~g} / \mathrm{L}$ Bactotryptone

- YPA: $20 \mathrm{~g} / \mathrm{L}$ Bactotryptone, $10 \mathrm{~g} / \mathrm{L}$ Yeast Extract, $0.04 \mathrm{~g} / \mathrm{L}$ adenine

- SC: $1.7 \mathrm{~g} / \mathrm{L}$ Yeast Nitrogen Base without Amino Acids \& Ammonium Sulfate, $5 \mathrm{~g} / \mathrm{L}$ Ammonium Sulfate, $2 \mathrm{~g} / \mathrm{L}$ Dropout amino acid mix, $0.8 \mathrm{~mL} / \mathrm{L} 4 \mathrm{M} \mathrm{NaOH}$

- Dropout amino acid mix: 2 g Adenine, 2 g L-Alanine, 2 g L-Arginine, 2 g LAsparagine, 2 g L-Aspartate, 2 g L-Cysteine, 2 g L-Glutamine, 2 g L-Glutamate, 2 g Glycine, 2 g L-Isoleucine, 2 g L-Lysine, 2 g L-Methionine, 2 g L-Phenylalanine, $2 \mathrm{~g}$ L-Proline, $2 \mathrm{~g}$ L-Serine, $2 \mathrm{~g}$ L-Threonine, $2 \mathrm{~g}$ L-Tyrosine, $2 \mathrm{~g}$ L-Valine, $0.2 \mathrm{~g}$ pABA (p-aminobenzoic acid)

- Carbon sources: 20 \% (w/v) of either Glucose, Galactose, Raffinose in $\mathrm{H}_{2} \mathrm{O}$, autoclaved except Raffinose (filtered sterile)

\subsubsection{Buffers}

- 10x DNA Loading Dye for agarose gels: $20 \%$ glycerol, $0.25 \%$ bromphenole blue, $0.25 \%$ xylene cyanol

- 10x TB-buffer: 890 mM Tris-Base, $890 \mathrm{mM}$ borate

- 10x TBE-buffer: 890 mM Tris-Base, 890 mM borate, 25 mM EDTA

- 10x PBS-buffer: $1.37 \mathrm{M} \mathrm{NaCl}, 27 \mathrm{mM} \mathrm{KCl}, 81 \mathrm{mM} \mathrm{Na}_{2} \mathrm{HPO}_{4}, 18 \mathrm{mM} \mathrm{KH} \mathrm{PO}_{4}$; $\mathrm{pH} 7.4$

· 10x TBS-buffer: 200mM Tris-Base, $1.37 \mathrm{M} \mathrm{NaCl}$, pH 7.6

\subsubsection{Antibiotics}

- 1000x Ampicillin: $100 \mathrm{mg} / \mathrm{ml}$ in $\mathrm{ddH}_{2} \mathrm{O}$; working concentration: $100 \mu \mathrm{g} / \mathrm{ml}$

- 1000x Chloramphenicol: $35 \mathrm{mg} / \mathrm{ml}$ in $70 \% \mathrm{EtOH}$; working concentration: 35 $\mu \mathrm{g} / \mathrm{ml}$

- 1000x Kanamycin: $50 \mathrm{mg} / \mathrm{ml}$ in $\mathrm{ddH}_{2} \mathrm{O}$ : working concentration: $50 \mu \mathrm{g} / \mathrm{ml}$

- 1000x Spectinomycin: $50 \mathrm{mg} / \mathrm{ml}$ in $\mathrm{ddH}_{2} \mathrm{O}$ : working concentration: $50 \mu \mathrm{g} / \mathrm{ml}$ 
2.1.2.5 SDS-PAGE and Western Blot buffers and solutions

- 4x Loading Buffer for SDS-PAGE: 50 mM Tris $\mathrm{HCl}$ pH 6.8, $100 \mathrm{mM}$ DTT, 2 $\%$ (w/v) SDS, $0.1 \%$ (w/v) bromphenole blue, $10 \%$ (v/v) glycerol

- 4x Loading Buffer for NuPage ${ }^{\circledR}$ Tris-Acetate and Bis-Tris gels: $0.564 \mathrm{M}$ Tris Base, $0.424 \mathrm{M}$ TrisHCl, 8 \% (w/v) SDS, $10.2 \mathrm{mM}$ EDTA, $0.88 \mathrm{mM}$ Brilliant Blue, $400 \mathrm{mM}$ DTT, $40 \%$ (v/v) gylcerol

- 10x SDS-PAGE Running Buffer: 250 mM Tris Base, 1.92 M Glycine, $1 \%$ SDS

- 20x Tris-Acetate Running Buffer: 1 M Tricine, 1 M Tris Base, 70 mM SDS

- 20x MOPS Running Buffer: 1 M MOPS, 1 M Tris Base, 70 mM SDS, 20.5 mM EDTA

- 1x Transfer Buffer for Western Blot: 25 mM Tris Base, $192 \mathrm{mM}$ Glycine, 0.1 \% SDS, $20 \%$ (v/v) Methanol

- 1x Transfer Buffer for NuPage ${ }^{\circledR}$ PAGE: 25 mM Bicine, 25 mM Bis-Tris, 1.05 mM EDTA, $1.3 \mathrm{mM}$ Sodium Bisulfite, $\mathrm{pH} 7.2$ (wo adjustments), $10 \%$ (v/v) Methanol

• 0.5 \% Ponceau S solution: $0.5 \%(w / v)$ Ponceau S in $5 \%(w / v)$ TCA

\subsubsection{Used Kits}

- Amersham ECL Plus ${ }^{\mathrm{TM}}$ Western Blotting Detection Kit (\#RPN2132)

- Amersham ECL Prime ${ }^{\mathrm{TM}}$ Western Blotting Detection Reagent (\#RPN2236)

- Amersham ECL Plex ${ }^{\mathrm{TM}}$ Western Blotting (\#RPN998)

- BigDye ${ }^{\circledR}$ Terminator v1.1 Cycle Sequencing Kit (\#4337451), Applied Biosystems

- peqGOLD Gel Extraction Kit (\#12-2500-02), PEQLAB

· peqGOLD Plasmid Miniprep Kit I (\#12-6943-02), PEQLAB

\subsubsection{Strains}

\subsubsection{Bacteria}

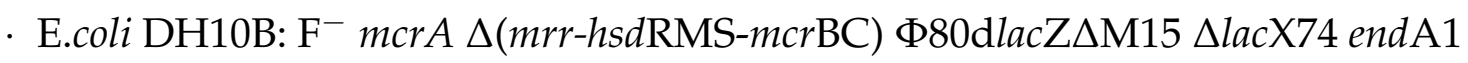
recA1 $\Delta$ (ara,leu)7697 araD139 galU galK nup GrpsL $\Lambda^{-}$

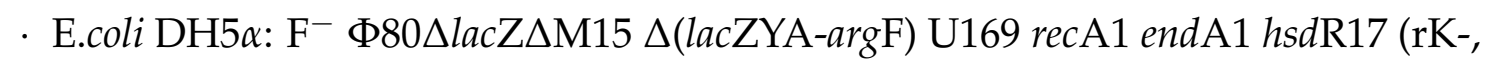
$\mathrm{mK}+)$ phoA sup E44 $\Lambda$ - thi-1 gyrA96 relA1

- E.coli BL21 (DE3): $\mathrm{F}^{-}$ompT $h s d \mathrm{~S}_{B}\left(\mathrm{r}_{B}{ }^{-} \mathrm{m}_{B}{ }^{-}\right) \mathrm{gal} d c m \lambda$ (DE3)

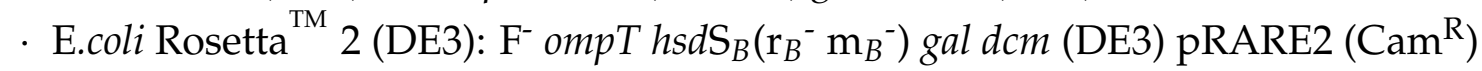




\subsubsection{Yeasts}

Table 2.1: Yeast strains used in this study - sequence verified mutations: pob3-L78R: L78R (CTACGA), N187D (AAC->GAC), N331D (AAT->GAT), E503D (GAA->GAT); spt16-ts: G132D (GGT->GAT), E1013G (GAA->GGA)

\begin{tabular}{|c|c|c|c|c|}
\hline strain & mutant & genotype & source & growth $\vartheta$ \\
\hline BY4741- $a$ & & MATa his3 $\Delta 1$ leu $2 \Delta 0$ met $15 \Delta 0$ ura3 $\Delta 0$ & AG Neumann & $30^{\circ} \mathrm{C}$ \\
\hline BY4741- $a$ & $\Delta \operatorname{trp}$ & $\begin{array}{l}\text { MATa his3 } \Delta 1 \text { leu } 2 \Delta 0 \text { met } 15 \Delta 0 \text { ura3 } \Delta 0 \\
\text { TRP1::KanMX }\end{array}$ & $\begin{array}{l}\text { kind gift from Prof. } \\
\text { Heike Krebber }\end{array}$ & $30^{\circ} \mathrm{C}$ \\
\hline BY4741- $a$ & H2A:3myc & $\begin{array}{l}\text { MATa his3 } \Delta 1 \text { leu } 2 \Delta 0 \text { met } 15 \Delta 0 \text { ura3 } \Delta 0 \\
\text { HTA1-3myc::HIS3 }\end{array}$ & AG Neumann & $30^{\circ} \mathrm{C}$ \\
\hline BY4741- $a$ & H2B:3myc & $\begin{array}{l}\text { MATa his3 } \Delta 1 \text { leu } 2 \Delta 0 \text { met } 15 \Delta 0 \text { ura3 } \Delta 0 \\
\text { HTB2-3myc::HIS3 }\end{array}$ & AG Neumann & $30^{\circ} \mathrm{C}$ \\
\hline BY4741- $a$ & $\mathrm{H} 4: 3 \mathrm{myc}$ & $\begin{array}{l}\text { MATa his } 3 \Delta 1 \text { leu } 2 \Delta 0 \text { met } 15 \Delta 0 \text { ura } 30 \\
\text { HHF1-3myc::HIS3 }\end{array}$ & AG Neumann & $30^{\circ} \mathrm{C}$ \\
\hline DSY5 & & $\begin{array}{l}\text { МAT } \alpha \text { leu2 trp1 ura3-52 his3::PGAL1-GAL4 } \\
\text { pep4 prb1-1122 }\end{array}$ & $\begin{array}{l}\text { kind gift from Prof. } \\
\text { Blanche Schwappach }\end{array}$ & $30^{\circ} \mathrm{C}$ \\
\hline $\begin{array}{l}\text { ATCC } \\
201388\end{array}$ & H2A:GFP & $\begin{array}{l}\text { MATa his } 3 \Delta 1 \text { leu } 2 \Delta 0 \text { met } 15 \Delta 0 \text { ura } \Delta 0 \\
\text { HTA2-GFP(S65T)::HIS3 }\end{array}$ & Huh et al., 2003279 & $30^{\circ} \mathrm{C}$ \\
\hline $\begin{array}{l}\text { ATCC } \\
201388\end{array}$ & H2B:GFP & $\begin{array}{l}\text { MATa his3 } \Delta 1 \text { leu } 2 \Delta 0 \text { met } 15 \Delta 0 \text { ura3 } \Delta 0 \\
\text { HTB1-GFP(S65T)::HIS3 }\end{array}$ & Huh et al., 2003279 & $30^{\circ} \mathrm{C}$ \\
\hline $\begin{array}{l}\text { ATCC } \\
201388\end{array}$ & H3:TAP & $\begin{array}{l}\text { MATa his3 } \Delta 1 \text { leu } 2 \Delta 0 \text { met } 15 \Delta 0 \text { ura } \Delta 0 \\
\text { HHT1-TAP::HIS3 }\end{array}$ & $\begin{array}{l}\text { Ghaemmaghami } \\
\text { et al., 2003 } 280\end{array}$ & $30^{\circ} \mathrm{C}$ \\
\hline $\begin{array}{l}\text { ATCC } \\
201388\end{array}$ & H4:GFP & $\begin{array}{l}\text { MATa his3 } \Delta 1 \text { leu } 2 \Delta 0 \text { met } 15 \Delta 0 \text { ura3 } \Delta 0 \\
\text { HHF2-GFP(S65T)::HIS3 }\end{array}$ & Huh et al., 2003 279 & $30^{\circ} \mathrm{C}$ \\
\hline $\begin{array}{l}\text { ATCC } \\
201388\end{array}$ & Spt16:GFP & $\begin{array}{l}\text { MATa his3 } \Delta 1 \text { leu } 2 \Delta 0 \text { met } 15 \Delta 0 \text { ura3 } \Delta 0 \\
\text { YGL207W-GFP(S65T)::HIS3 }\end{array}$ & Huh et al., 2003 & $30^{\circ} \mathrm{C}$ \\
\hline $\begin{array}{l}\text { ATCC } \\
201388\end{array}$ & Pob3:GFP & $\begin{array}{l}\text { MATa his } 3 \Delta 1 \text { leu } 2 \Delta 0 \text { met15 } \Delta 0 \text { ura3 } \Delta 0 \\
\text { YML069W-GFP(S65T)::HIS3 }\end{array}$ & Huh et al., 2003 279 & $30^{\circ} \mathrm{C}$ \\
\hline BY4741- $a$ & pob3-L78R & $\begin{array}{l}\text { MATa his3 } \Delta 1 \text { leu } 2 \Delta 0 \text { met } 15 \Delta 0 \text { ura3 } \Delta 0 \\
\text { pob3 } \Delta:: P O B 3 L 78 R: k a n M X\end{array}$ & Li et al., $2011^{281}$ & $25^{\circ} \mathrm{C}$ \\
\hline BY4741- $a$ & spt16-ts & $\begin{array}{l}\text { MATa his } 3 \Delta 1 \text { leu } 2 \Delta 0 \text { met } 15 \Delta 0 \text { ura } 300 \\
\text { spt16 } \Delta:: S P T 16-t s: k a n M X\end{array}$ & Li et al., $2011^{281}$ & $25^{\circ} \mathrm{C}$ \\
\hline MY721- $\alpha$ & & $\begin{array}{l}\text { MAT } \alpha \text { his } 3 \Delta 1 \text { leu2 } \Delta 0 \text { LYS2+ met } 15 \Delta 0 \\
\text { ura3 } \Delta 0 \text { can } 1 \Delta:: \text { STE2pr-spHIS5 } \\
\text { lyp } 1 \Delta:: \text { STE3pr-LEU2 }\end{array}$ & $\begin{array}{l}\text { A H Tong et al., } 2001 \\
\text { 2006, 2007282 }\end{array}$ & $30^{\circ} \mathrm{C}$ \\
\hline
\end{tabular}

\subsubsection{Used chromatography material and columns:}

- Glutathione Sepharose 4B (\#17-0756-01), GE Healthcare

- HiLoad ${ }^{\circledR} 26 / 60$ Superdex ${ }^{\circledR}, 75$ PG, (\#28-9893-34), GE Healthcare

- HisTrap ${ }^{\text {TM }}$ FF 5 mL column (\#17-5255-01), GE Healthcare

- Ni-NTA Superflow Cartridge (5 mL),(\#30760), Qiagen

. Superdex ${ }^{\text {TM }} 200$ 10/300 GL (\#17-5175-01), GE Healthcare

- Thermo Scientific ${ }^{\mathrm{TM}}$ HisPur $^{\mathrm{TM}}$ Ni-NTA Resin (\#PI-88222), Thermo Scientific 


\subsubsection{Antibodies}

The antibodies used in this thesis are shown in table 2.2. Common working concentration and solution are indicated.

Table 2.2: Antibodies used in this study - WC: working concentration; *: TBS or PBS varied upon primary antibody solution

\begin{tabular}{|c|c|c|c|c|}
\hline antibody & species & source & common WC & solution \\
\hline \multicolumn{5}{|l|}{ primary antibodies } \\
\hline$\alpha$-c-Myс (9E10) & mouse & Millipore (05-419) & 1:5.000 & $5 \%$ MLK/TBS \\
\hline$\alpha$-c-Myc (9E10) & mouse & Santa Cruz Biotechnology (sc-40) & 1:1.000 & $5 \% \mathrm{MLK} / \mathrm{TBS}$ \\
\hline$\alpha-\mathrm{HA}$ & rabbit & abcam (ab9110) & 1:10.000 & $3 \%$ BSA $/ \mathrm{PBS}$ \\
\hline$\alpha$-PGK1 & mouse & Invitrogen (A6457) & 1:5.000 & $3 \% \mathrm{BSA} / \mathrm{TBS}$ \\
\hline$\alpha-\mathrm{H} 2 \mathrm{~A}$ & rabbit & abcam (ab13923) & 1:3.000 & $3 \%$ MLK/TBS \\
\hline$\alpha-\mathrm{H} 2 \mathrm{~B}$ & rabbit & abcam (ab1790) & $1: 3.000$ & $3 \%$ MLK/TBS \\
\hline$\alpha-\mathrm{H} 3$ & rabbit & abcam (ab1791) & $1: 2.500$ & $3 \% \mathrm{BSA} / \mathrm{TBS}$ \\
\hline$\alpha-\mathrm{H} 4$ & rabbit & abcam (ab7311) & $1: 500$ & $3 \% \mathrm{BSA} / \mathrm{TBS}$ \\
\hline$\alpha$-HIS & mouse & GE Healthcare (27-4710-01) & 1:3.000 & $3 \%$ BSA $/ \mathrm{PBS}$ \\
\hline \multicolumn{5}{|l|}{ secondary antibodies } \\
\hline$\alpha$-mouse-IgG-HRP & goat & SIGMA-ALDRICH (A4416) & 1:10.000 & $5 \%$ MLK$^{*}$ \\
\hline$\alpha$-rabbit-IgG-HRP & goat & SIGMA-ALDRICH (A6154) & 1:10.000 & $5 \% \mathrm{MLK}^{*}$ \\
\hline$\alpha$-mouse-IgG-Cy3 & goat & GE Healthcare (PA43010) $1 \mu \mathrm{g} / \mu \mathrm{L}$ & $1: 2.500$ & $5 \%$ MLK/TBS \\
\hline
\end{tabular}

\subsubsection{Enzymes and DNA Ladder}

• DNaseI 2 mg/ml [4566.2 U/mg] (\#A3778,0100), AppliChem

- Fermentas (Thermo Scientific) Restriction Endonucleases and Buffers/Supplements

- GeneRuler ${ }^{\mathrm{TM}}$ 100bp Plus DNA Ladder (\#SM0321), Thermo Scientific

- GeneRuler ${ }^{\mathrm{TM}} 1 \mathrm{~kb}$ DNA Ladder (\#SM0312), Thermo Scientific

- Lysozyme from hen egg white crystallized (Hydrochloride) [79110 E/mg], Boehringer Mannheim

- PageRuler ${ }^{\mathrm{TM}}$ Prestained Protein Ladder (\#26616), Thermo Scientific

- Amersham ECL Plex ${ }^{\mathrm{TM}}$ Fluorescent Rainbow Markers (\#RPN850E), GE Healthcare

- PfuTurbo ${ }^{\circledR}$ DNA Polymerase (\#600250) with 10x C-Pfu Reaction Buffer, Agilent

- Phusion High-Fidelity DNA Polymerase 2 U/ $\mu$ L (\#F-530S) with 5x Phusion ${ }^{\circledR}$ HF Reaction Buffer [7.5 mM MgCl 2 ], Thermo Scientific

- Shrimp Alkaline Phosphatase (SAP) (\#EF0511), Fermentas

- CIAP (Calf Intestine Alkaline Phosphatase) (\#EF0341) with 10x Reaction Buffer, Fermentas

. T4 DNA Ligase (\#EL0014) with T4 DNA Ligase Reaction Buffer, Thermo Scientific 
A.

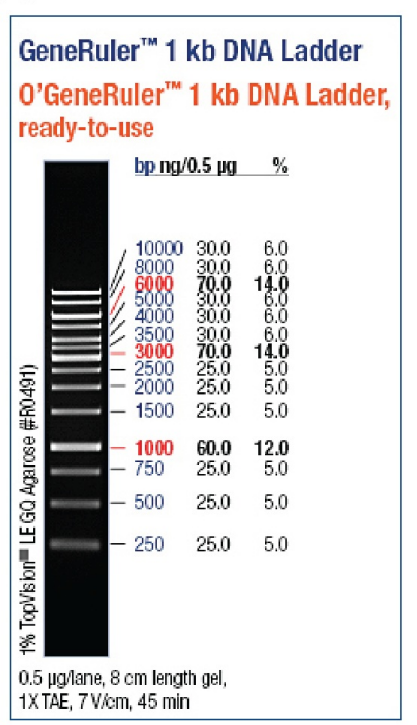

B.

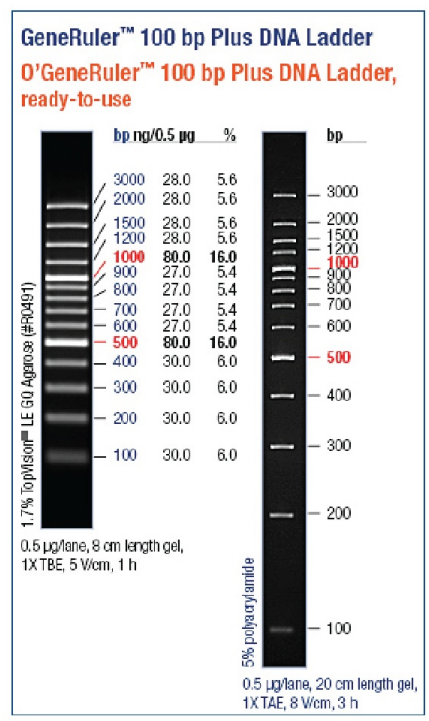

C.

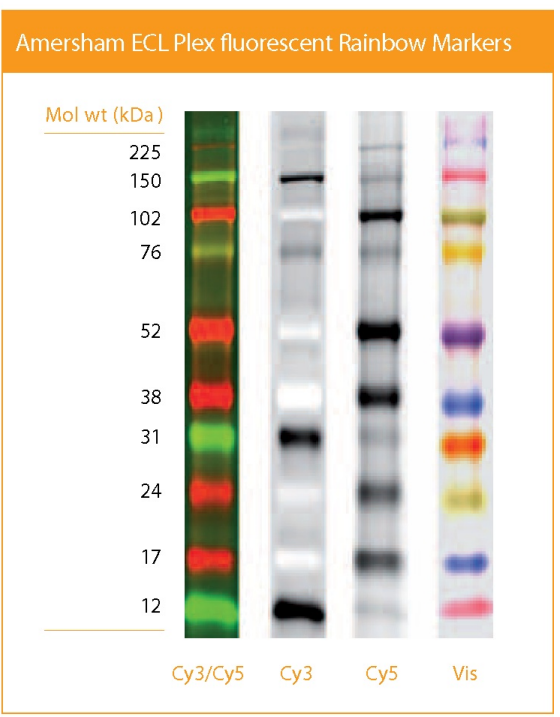

D.

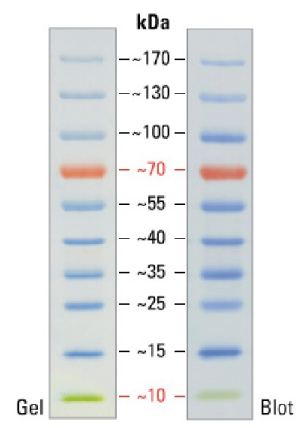

E.

\begin{tabular}{|c|c|c|c|c|c|}
\hline & I type & Tris-Gl & lycine & Tris-Acetate & Bis-Tris \\
\hline Gel c & centration & $12 \%$ & $15 \%$ & $3-8 \%$ & $4-12 \%$ \\
\hline Runr & g buffer & Tris-Gl & ycine & Tris-Acetate & MOPS \\
\hline & & & Apparen & Molecular Sizes & \\
\hline & 10 & $={ }_{130}^{170}$ & $\overline{\overline{\bar{C}}} \begin{array}{l}170 \\
30 \\
30\end{array}$ & & \\
\hline & 20 & $\bar{Z}_{70}^{100}$ & — 70 & & \\
\hline & $\frac{30}{40}$ & $\begin{array}{r}55 \\
5 \\
40\end{array}$ & $\begin{array}{r}\square \\
\end{array}$ & -150 & $\square^{2}$ \\
\hline $\mathrm{d}$ & 50 & ${ }^{-} 35$ & $\begin{array}{r}{ }^{3} 5 \\
25\end{array}$ & -120 & $\begin{array}{l}{ }^{2} \\
5 \\
5\end{array}$ \\
\hline E & 60 & $\longrightarrow 25$ & & ${ }^{85}$ & ${ }^{-} 4$ \\
\hline & 80 & -15 & -15 & $\begin{array}{l}{ }^{65} \\
50\end{array}$ & ${ }^{3}$ \\
\hline & $\frac{90}{100}$ & -10 & 10 & $\begin{array}{r}{ }^{40} \\
30\end{array}$ & -1 \\
\hline
\end{tabular}

Figure 2.1: Migration patterns of DNA and Protein ladders: A: GeneRuler ${ }^{\mathrm{TM}} 1 \mathrm{~kb}$ DNA Ladder; B: GeneRuler ${ }^{\mathrm{TM}} 100 \mathrm{bp}$ Plus DNA Ladder ; C: Amersham ECL Plex ${ }^{\mathrm{TM}}$ Fluorescent Rainbow Marker; D: PageRuler ${ }^{\text {TM }}$ Prestained Protein Ladder (4-20 \% Tris-glycine gel, SDS-PAGE); E: PageRuler ${ }^{\mathrm{TM}}$ Prestained Protein Ladder modified from Thermo Scientific; images are taken or modified from company websites 


\subsubsection{Software}

- Adobe ${ }^{\circledR}$ Photoshop ${ }^{\circledR}$ CS5.1, Adobe Systems

- Adobe ${ }^{\circledR}$ Illustrator ${ }^{\circledR}$ CS5.1, Adobe Systems

- Geneious 6.0.5 (trial), Biomatters Ltd.

- ImageJ $1.49 \mathrm{a}^{283}$

- Fiji 284

- Plot 2.0 for Mac, Michael Wesemann

- PyMOL X11 Hybrid 0.99rc6 for Mac, DeLano Scientific LLC.

\subsubsection{Vectors}

- pUG35 pMET yEGFP URA3 ${ }^{285}$

- pUG35 pMET Pob3:yEGFP3

- pESC pBPA-RS 255

- pESC pAzF-RS 255

- pSUP pBPA 233

- pRS426 pGALS

- pRS426

- p426 GAL 3HA

- p426 GAL Pob3:3HA

- p426 GAL 9myc

- p426 GAL Pob3:9myc

- p426 GAL Pob3:9myc XTAG

- p426 E Spt16:9myc

- p426 E Spt16:9myc XTAG

- pCDF DUET-1, Novagen ${ }^{\circledR}$

- pRSF DUET-1, Novagen ${ }^{\circledR}$

- pCDFD His-ctPob3

- pCDFD His-TEV-ctPob3

- pCDFD His-TEV-ctPob3 $\triangle$ D467-G571

- pRSFD His-TEV-ctPob3

- pRSFD His-TEV-ctPob3 XTAG

- pCDFD His-TEV-yNhp6a

- pUG35 pMET Pob3 $\triangle 458-552: y E G F P 3$

- pUG35 pMET Pob3 $5444-552: y E G F P 3$

- pUG35 pMET Pob3K547M:yEGFP3

- pUG35 pMET Pob3 4 458-543:yEGFP3

- pGEX6P1 hImp- $\alpha \Delta$ IBB (kind gift from Dr. Achim Dickmanns) 


\subsection{Methods}

\subsubsection{DNA methods}

\subsubsection{Isolation of genomic DNA from Saccharomyces cerevisiae}

Isolation of genomic DNA from several Saccharomyces cerevisiae strains was performed combining two protocols: Spheroplasting of yeast cells and subsequent genomic DNA isolation by sodium acetate ethanol precipitation using solutions from the peqGOLD Plasmid Miniprep Kit. Cells from 5-10 mL of a fresh yeast overnight culture were collected by centrifugation and resuspended in $250 \mu \mathrm{L}$ buffer P1 substituted with lyticase (final concentration $10 \mathrm{U} / \mathrm{mL}$ ). Cells were incubated at $37^{\circ} \mathrm{C}$ for 30 minutes. Subsequently, $250 \mu \mathrm{L}$ lysis buffer P2 and $60 \mu \mathrm{L}$ of a $3 \mathrm{M}$ potassium acetate solution ( $\mathrm{pH}$ 5.2) was added. The samples were incubated on ice for 30 minutes and centrifuged at $13.000 \mathrm{rpm}$ in a table-top centrifuge for 3 minutes. The soluble supernatant was transferred to a fresh $2 \mathrm{~mL}$ tube and mixed with $1 \mathrm{~mL} 100 \%$ ethanol (two times the volume) by inverting the cap. After incubation at room temperature for 5 minutes, the precipitate was collected by centrifugation at $13.000 \mathrm{rpm}$ for 5 minutes and the supernatant was discarded. The pellet was washed with $1 \mathrm{~mL} 70 \%$ ethanol and collected by centrifugation as described above. The supernatant was discarded and the pellet was dried using a SpeedVac (2 minutes). Finally, the pellet was resuspended in $100 \mu \mathrm{L}$ elution buffer and the nucleic acid concentration was determined by $\mathrm{UV}$ spectroscopy at $260 \mathrm{~nm}$. The concentration was adjusted to $100 \mathrm{ng} / \mu \mathrm{L}$ (common working concentration) and the DNA was stored at $-20^{\circ} \mathrm{C}$.

\subsubsection{Primer design}

Primers were designed using the software Geneious 6.0.5 and purchased from Sigma Aldrich. Primers were dissolved in $\mathrm{ddH}_{2} \mathrm{O}(100 \mu \mathrm{M}$ stock concentration) and stored at $-20^{\circ} \mathrm{C}$. All primers used in this study are shown in the appendix tables 5.2 (amber library primers) and 5.1 (general cloning and sequencing primers).

\subsubsection{PCR amplification}

For PCR amplification of target sequences from genomic DNA or plasmids, the Phusion ${ }^{\circledR}$ High-Fidelity DNA Polymerase was used. Reactions were prepared in $0.2 \mathrm{~mL}$ PCR tubes on ice. Final primer concentration was $0.2 \mu \mathrm{M}$. For amplification from genomic DNA approximately $100 \mathrm{ng}$ template DNA was used. All components were mixed and transferred to a PCR cycler. The standard pipetting scheme and 
PCR program is shown in table 2.3. After amplification, reactions were analyzed by agarose gelelectrophoresis 2.2.1.10).

Table 2.3: Pipetting scheme and PCR program for standard PCR amplification -

\begin{tabular}{lc}
\hline component & amount in $\mu \mathbf{L}$ \\
\hline $\mathrm{ddH}_{2} \mathrm{O}$ & 35 \\
$5 x$ Phusion ${ }^{\circledR}$ HF Reaction Buffer & 10 \\
\hline $\mathrm{dNTP} \operatorname{mix}(10 \mathrm{mM}$ each) & 1.5 \\
primer (S) $10 \mathrm{pmol} / \mu \mathrm{L}$ & 1 \\
primer (AS) $10 \mathrm{pmol} / \mu \mathrm{L}$ & 1 \\
\hline template DNA & 1 (approx. $100 \mathrm{ng})$ \\
Phusion ${ }^{\circledR} \mathrm{HF}$ DNA Polymerase & $0.5(1 \mathrm{U})$ \\
total volume & 50 \\
\hline
\end{tabular}

\begin{tabular}{ccr}
\hline temperature & time & cycles \\
\hline $98^{\circ} \mathrm{C}$ & $3^{\prime}$ & 1 \\
\hline $98^{\circ} \mathrm{C}$ & $10^{\prime \prime}$ & \\
$56^{\circ} \mathrm{C}$ & $30^{\prime \prime}$ & 25 \\
$72^{\circ} \mathrm{C}$ & $15^{\prime \prime}-30^{\prime \prime} / \mathrm{kb}$ & \\
\hline $72{ }^{\circ} \mathrm{C}$ & $10^{\prime}$ & 1 \\
\hline $4{ }^{\circ} \mathrm{C}$ & $\infty$ & 1 \\
\hline
\end{tabular}

\subsubsection{Quikchange mutagenesis PCR:}

Quikchange mutagenesis PCR was performed to introduce changes (exchange, insertion or deletion) in DNA sequence of template plasmids. Primers are designed in an overlapping fashion covering the region of interest: $10 \mathrm{bp}$ upstream and at least $17 \mathrm{bp}$ downstream of the region are complementary to the plasmid template. After PCR amplification, the methylated parent plasmid was digested with $10 \mathrm{U} D p n I$ for at least 1 hour at $37^{\circ} \mathrm{C}$. Subsequently, reactions were directly used for transformation of chemical competent bacteria (2.2.1.7) .

Table 2.4: Pipetting scheme and PCR program for Quikchange reactions using PfuTurbo ${ }^{\circledR}$ DNA Polymerase

\begin{tabular}{lc}
\hline component & amount in $\mu \mathbf{L}$ \\
\hline $\mathrm{ddH}_{2} \mathrm{O}$ & 16 \\
10x Cloned Pfu Reaction Buffer & 2.0 \\
\hline $\mathrm{dNTP} \operatorname{mix}(10 \mathrm{mM}$ each) & 0.4 \\
primer (S) $10 \mathrm{pmol} / \mu \mathrm{L}$ & 0.4 \\
primer (AS) $10 \mathrm{pmol} / \mu \mathrm{L}$ & 0.4 \\
\hline template DNA & 0.4 (approx. 50-100 ng) \\
PfuTurbo $^{\circledR}$ DNA Polymerase & $0.4(1 \mathrm{U})$ \\
total volume & 20 \\
\hline
\end{tabular}

\begin{tabular}{ccc}
\hline temperature & time & cycles \\
\hline $95^{\circ} \mathrm{C}$ & $3^{\prime}$ & 1 \\
\hline $95^{\circ} \mathrm{C}$ & $30^{\prime \prime}$ & \\
$58^{\circ} \mathrm{C}$ & $30^{\prime \prime}$ & 17 \\
$72^{\circ} \mathrm{C}$ & $60^{\prime \prime} / \mathrm{kb}$ & \\
\hline $72^{\circ} \mathrm{C}$ & $10^{\prime}$ & 1 \\
\hline $4^{\circ} \mathrm{C}$ & $\infty$ & 1 \\
\hline
\end{tabular}


Table 2.5: Pipetting scheme and PCR program for Quikchange reactions using Phusion ${ }^{\circledR}$ DNA Polymerase

\begin{tabular}{lc}
\hline component & amount in $\mu \mathbf{L}$ \\
\hline ddH $_{2} \mathrm{O}$ & 12.8 \\
$5 x$ Phusion ${ }^{\circledR}$ HF Reaction Buffer & 4 \\
\hline $\mathrm{dNTP} \operatorname{mix}(10 \mathrm{mM}$ each) & 0.5 \\
primer (S) $10 \mathrm{pmol} / \mu \mathrm{L}$ & 1 \\
primer (AS) $10 \mathrm{pmol} / \mu \mathrm{L}$ & 1 \\
template DNA & 0.5 (approx. $50-100 \mathrm{ng})$ \\
Phusion ${ }^{\circledR}$ DNA Polymerase & $0.2(0.4 \mathrm{U})$ \\
total volume & 20 \\
\hline
\end{tabular}

\begin{tabular}{ccc}
\hline temperature & time & cycles \\
\hline $98^{\circ} \mathrm{C}$ & $5^{\prime}$ & 1 \\
\hline $98^{\circ} \mathrm{C}$ & $10^{\prime \prime}$ & \\
$56^{\circ} \mathrm{C}$ & $30^{\prime \prime}$ & 17 \\
$72^{\circ} \mathrm{C}$ & $15^{\prime \prime}-30^{\prime \prime} / \mathrm{kb}$ & \\
\hline $72^{\circ} \mathrm{C}$ & $10^{\prime}$ & 1 \\
\hline $4^{\circ} \mathrm{C}$ & $\infty$ & 1 \\
\hline
\end{tabular}

\subsubsection{Restriction digests}

Class-II restriction enzymes are recognizing their specific palindromic recognition site and perform cleavage of the phosphodiester bond of the DNA. Restriction digests were performed either in a preparative or an analytical scale. For an analytical digest proximately $300 \mathrm{ng}$ plasmid DNA was used and analyzed for fragment patterning by agarose gelelectrophoresis (2.2.1.10). For molecular cloning, preparative digests were performed to produce compatible ends of PCR products and vector plasmid backbones. Typically, $1 \mu \mathrm{g}$ of vector plasmid was digested with $10 \mathrm{U}$ of restriction enzyme for at least 1 hour. Buffers, supplements and incubation temperature were chosen accordingly to manufacturer's instructions for each restriction enzyme. Double digests conditions were examined using the DoubleDigest tool from Thermo Scientific (http:/ / www.thermoscientificbio.com/webtools/doubledigest/). Prior to PCR digest, the product was purified from the PCR mixture using the peqGOLD Gel Extraction Kit according to manufacturer's instructions.

\subsubsection{Ligation of DNA molecules}

In molecular biology, the term ligation stands for the formation of a covalent phosphodiester bond between DNA fragments which posses a free 3' hydroxyl and a 5' phosphate group in their backbone. Ligases are enzymes catalyzing this reaction. Ligations can be performed between compatible ends of DNA molecules such as digested PCR fragments and vector backbones. For a standard ligation, $1 \mathrm{U}$ of the bacteriophage T4 DNA Ligase was used in a total volume of $10 \mu \mathrm{L}$. Usually, $50 \mathrm{ng}$ of digested vector were mixed with a 3 molar excess of insert DNA (molar insert:vector ratio 3:1). In cases where the insert amount could not be determined, $50 \mathrm{ng}$ of digested vector with the maximum amount of insert was used. Reactions were carried 
out for 1 hour at $37^{\circ} \mathrm{C}$ or overnight at room temperature.

\subsubsection{Transformation of bacteria}

A standard calcium chloride protocol was used to achieve chemical competent DH10B or DH5 $\alpha$ Escherichia coli cells. These cells are able to take up DNA from their environment upon a heat shock. This ability is used to transform Escherichia coli cells with DNA of interest. Ligation reactions $(5-10 \mu \mathrm{L})$ or purified plasmids (10$100 \mathrm{ng}$ ) were mixed with thawed competent cells on ice (ratio 1:10 (v/v)). The mixture was kept on ice for 10 minutes and subsequently subjected to heat shock for 2 minutes at $42{ }^{\circ} \mathrm{C}$. After heatshock, the cells were incubated for another 10 minutes on ice. Recovery was performed in $700 \mu \mathrm{L}$ of LB medium without antibiotics and incubated at $37{ }^{\circ} \mathrm{C}$ in a thermomixer $(800 \mathrm{rpm})$ for approximately 45 minutes. Following recovery, $150 \mu \mathrm{L}$ were spread on agar-plates containing the appropriate antibiotics for selection (working concentrations 2.1.2.4). The residual cells were pelleted by centrifugation and spread on a second agar plate with appropriate antibiotics. Plates were incubated at $37^{\circ} \mathrm{C}$ until colonies were grown. Clones were further subjected to preparation of plasmids (2.2.1.8).

\subsubsection{Minipreparation of plasmids}

Preparation of plasmid DNA from Escherichia coli was done using peqGOLD Plasmid Miniprep Kit according to manufacturer's instructions. Bacterial colonies were incubated in $4 \mathrm{~mL}$ LB media containing appropriate antibiotics. Cultures were kept at $37^{\circ} \mathrm{C}$ in a shaker (approx. $210 \mathrm{rpm}$ ) for at least 16 hours. Cells were harvested by centrifugation in a table-top centrifuge at full speed. The cell pellet was subjected to plasmid preparation as described in the manual. The purification principle is based upon the DNA-property of binding to silica gel under high chaotropic salt conditions and subsequent elution under low salt conditions. The DNA was finally eluted with $50 \mu \mathrm{L}$ elution buffer and concentration was determined using UV spectroscopy at $260 \mathrm{~nm}$. Plasmids were stored at $-20^{\circ} \mathrm{C}$ or subjected to downstream methods such as restriction digest or sequencing.

\subsubsection{Sequencing}

DNA sequencing was performed at three different facilities. Low quantities sequencing reactions, where results were not needed rapidly were prepared for in-house facility after a modified chain-termination method invented by Sanger et al., 1977286 Therefore, the PCR reactions were set up by the user with the BigDye ${ }^{\circledR}$ Terminator 
v1.1 Cycle Sequencing Kit. This kit contains all necessary PCR reaction components including fluorescent labeled dideoxynucleotide triphosphates (ddNTPs). A standard sequencing pipetting scheme is shown in table 2.6. The reaction was purified using a standard sodium acetate ethanol precipitation with $1 \mu \mathrm{L}$ of $125 \mathrm{mM}$ EDTA ( $\mathrm{pH}$ 5.2), $1 \mu \mathrm{L} 3 \mathrm{M}$ sodium acetate and $50 \mu \mathrm{L} 100 \%$ ethanol. Precipitated PCR products were collected by centrifugation for 15 minutes at 13,200 rpm in a microcentrifuge. A washing step with $70 \mu \mathrm{L}$ of $70 \%$ ethanol was performed and the pellet was dried using a SpeedVac. The final pellet was suspended in $15 \mu \mathrm{L} 99.5 \%$ formamide and the sequencing reaction was analyzed using an ABI PRISM ${ }^{\circledR} 3100$ DNA capillary Sequencer.

Table 2.6: Pipetting scheme and PCR program of a standard sequencing reaction using the BigDye ${ }^{\circledR}$ Terminator v1.1 Cycle Sequencing Kit - BD: BigDye ${ }^{\circledR}$ Sequencing mix; RB: Reaction buffer

\begin{tabular}{lc}
\hline component & amount in $\mu \mathbf{L}$ \\
\hline primer 10 pmol $/ \mu \mathrm{L}$ & $0.8(8 \mathrm{pmol})$ \\
BD-Seq Mix & 1.0 \\
RB-Seq buffer & 1.0 \\
\hline template DNA & $200-400 \mathrm{ng}$ \\
${\mathrm{d} \mathrm{H}_{2} \mathrm{O} \text { to total volume }} }$ & 10 \\
\hline
\end{tabular}

\begin{tabular}{ccc}
\hline temperature & time & cycles \\
\hline $96^{\circ} \mathrm{C}$ & $10^{\prime \prime}$ & \\
$55^{\circ} \mathrm{C}$ & $15^{\prime \prime}$ & 25 \\
$60^{\circ} \mathrm{C}$ & $4^{\prime}$ & \\
\hline
\end{tabular}

High quantities of sequencing reactions, such as the Amber mutant library sequencing, were preformed by sequencing in a 96 well format using the Microbiology sequencing facility of Prof. Rolf Daniel (University of Göttingen). Therefore, only plasmid DNA and primer was mixed and transferred to a 96 well PCR plate. Overnight, sequencing reactions were done using the sequencing service provided by SEQLAB in Göttingen or GATC Biotech.

For verification of genomically modified yeast strains, regions were either subcloned in standard plasmids and subjected to sequencing or PCR products were directly sequenced after PCR-purification as described in 2.2.1.11.

\subsubsection{Agarose gelelectrophoresis}

Gel electrophoresis can be either performed as a preparative gel, used for isolating DNA fragments of interest, or as an analytical gel utilized for the analysis of restriction patterns of test digests. The overall negative charge of DNA which is proportional to the molecular weight and thereby length of the DNA, can be used to separate a mixture of nucleic acid fragments in a solidified gel matrix made out of agarose. Applying a vectored electric field to the matrix results in the separation 
of fragments of different size due to different migration distances. The fragments can be stained with fluorescent dyes which are present in the solidified gel matrix. Bands can be visualized by illumination of these dyes and pictures can be taken with a CCD-camera. The agarose concentration of the solidified gel matrix determines the a suitable separation range of nucleic acids (s. table 2.7). According to this

Table 2.7: Agarose concentrations and suitable separation lengths used in gelelectrophoresis (modified after ${ }^{287}$ )

\begin{tabular}{lc}
\hline separation range & $\begin{array}{c}\text { agarose } \\
\text { concentration }(\mathrm{w} / \mathrm{v})\end{array}$ \\
\hline $1-30 \mathrm{~kb}$ & $0.5 \%$ \\
$0.8-12 \mathrm{~kb}$ & $0.7 \%$ \\
$0.5-7 \mathrm{~kb}$ & $1.0 \%$ \\
$0.4-6 \mathrm{~kb}$ & $1.2 \%$ \\
$0.2-3 \mathrm{~kb}$ & $1.5 \%$ \\
$0.1-2 \mathrm{~kb}$ & $2.0 \%$ \\
\hline
\end{tabular}

table, an agarose/0.5xTBE solution was boiled in a microwave until the agarose was completely dissolved. The solution was cooled down and $4 \mu \mathrm{L}$ of GelRed Nucleic Acid Stain (10,000x) per $100 \mathrm{~mL}$ molten agarose solution was added. After mixing, the solution was poured into the gel-casting unit. The solidified gel was either used directly for electrophoresis with $0.5 x$ TBE buffer or stored wrapped in cling film at $4^{\circ} \mathrm{C}$. Samples were substituted with DNA Loading Dye to $1 \mathrm{x}$ final concentration. For size determination commercially available DNA standards were used (figure 2.1). Running conditions (voltages and times) were adapted to different gel casts, agarose percentages and separation lengths. Gel analysis and imaging was using with the GelDoc $^{\text {TM }} 2000$ from Bio-Rad.

\subsubsection{Extraction of DNA from agarose gel}

In case of a preparative electrophoresis the band of the agarose gel was cut out and purified with the peqGOLD Gel Extraction Kit. The DNA fragment of the agarose gel was excised with a clean and sharp scalpel. The weight of the gel piece was determined, mixed with an equal volume of Binding Buffer (100 mg 100 $\mu \mathrm{L})$ and dissolved at $55^{\circ} \mathrm{C}$ using a thermomixer. Binding of DNA, washing and elution were performed according to manufacturer's instructions. The DNA was eluted with elution buffer and subjected to downstream applications such as restriction digest or ligation.

The peqGOLD Gel Extraction Kit was used for purification of restrictions digests, as well as for PCR reactions. Therefore a PCR reaction was mixed with an equal 
volume of Binding Buffer and purified as describe above. The elution volume varied due to different downstream applications.

\subsubsection{Molecular cloning - Plasmid creations}

Subcloning of Genetic code expansion plasmids: The TRP1 auxotrophy marker cassette was excised from pESC pBPA-RS and pESC pAzF-RS with SacI and PfoI and compatible markers were introduced by the same restriction enzymes. The source of the auxotrophy markers is the pRS42X vector series. Successful cloning was confirmed by sequencing of the auxotrophy marker and analytical restriction digests (table 2.8). This cloning strategy removed the ADH terminator of the pBPA-RS. This was observed not to alter the genetic code expansion system negatively.

Table 2.8: Auxotrophy subcloning of the pESC pBPA-RS TRP and pESC pAzF-RS TRP plasmid 255 - The auxotrophy insert was ligated to the SacI and PfoI digested pESC pBPA-RS backbone or pESC pAzF-RS backbone $(6,606 \mathrm{bp})$ resulting in pBPA/pAzF-synthetase plasmids with different auxotrophy markers.

\begin{tabular}{cccc}
\hline $\begin{array}{c}\text { auxotrophy source } \\
\text { plasmid }\end{array}$ & auxotrophy marker & $\begin{array}{c}\text { length of insert (bp) } \\
\text { SacI and } \text { PfoI }\end{array}$ & final plasmid \\
\hline pRS423 & HIS3 & 2,136 & pESC pBPA-RS HIS \\
pRS425 & LEU2 & 3,187 & pESC pBPA-RS LEU \\
pRS426 & URA3 & 2,064 & pESC pBPA-RS URA \\
pRS425 & LEU2 & 3,187 & pESC pAzF-RS LEU \\
\hline
\end{tabular}

Construction of C-terminal tagged yeast expression plasmids: A series of yeast expression plasmids were created which allowed C-terminal tagging of open reading frames of interest. The basis for the plasmids were the pRS426 plasmid (pRS426 $\left[\text { ATCC }{ }^{\circledR} 77107^{\mathrm{TM}} \text { ] }\right)^{288}$ and the pRS426 pGAL plasmid (p426 GALS [ATCC ${ }^{\circledR} 87349^{\mathrm{TM}}$ ]) $)^{289}$ which contains a derived GAL1 promoter (GALS). The tags (3HA, 6HA, 3myc and $9 \mathrm{myc}$ ) were amplified by PCR from the pYM vector series ${ }^{290}$. The tags were amplified by PCR and cloned using SalI and XhoI restriction sites (table 2.9). The resulting plasmid were checked by analytical restriction digest and sequencing. 
Table 2.9: Cloning strategy for the C-terminal tag vector series - The protein tags were amplified from the epitope-tagging pYM plasmid series and cloned into yeast expression plasmids using SalI and XhoI restriction sites.

\begin{tabular}{|c|c|c|c|c|c|}
\hline PCR primers & Vector 290 & Tag & product size (bp) & $\begin{array}{c}\text { cloned into } \\
\text { (SalI and XhoI) }\end{array}$ & final plasmid \\
\hline \#CHR0144 + \#CHR0145 & pYM2 & 3HA & 150 & $\begin{array}{c}\text { pRS426 } \\
\text { p426 GAL }\end{array}$ & $\begin{array}{l}\text { pRS426 3HA } \\
\text { p426 GAL 3HA }\end{array}$ \\
\hline \#CHR0146 + \#CHR0147 & pYM4 & 3 myc & 165 & $\begin{array}{l}\text { pRS426 } \\
\text { p426 GAL }\end{array}$ & $\begin{array}{l}\text { pRS426 3myc } \\
\text { p426 GAL 3myc }\end{array}$ \\
\hline \#CHR0148 + \#CHR0149 & pYM3 & $6 \mathrm{HA}$ & 239 & $\begin{array}{l}\text { pRS426 } \\
\text { p426 GAL }\end{array}$ & $\begin{array}{l}\text { pRS426 6HA } \\
\text { p426 GAL 6HA }\end{array}$ \\
\hline \#CHR0148 + \#CHR0149 & pYM6 & 9 myc & 417 & $\begin{array}{c}\text { pRS426 } \\
\text { p426 GAL }\end{array}$ & $\begin{array}{l}\text { pRS426 9myc } \\
\text { p426 GAL 9myc }\end{array}$ \\
\hline
\end{tabular}

Cloning of Spt16 and Pob3 parent plasmids: The open reading frames of Spt16 (SPT16/YGL207W on chromosome VII from coordinates 98969 to 102076) and Pob3 (POB3/YML069W on chromosome XIII from coordinates 135500 to 137158) were amplified from genomic yeast DNA (genotype: W303) by PCR. For Spt16, the amplicon contained the endogenous promoter by inclusion of $500 \mathrm{bp}$ upstream of the start codon (coordinates 98469 to 102076). For Pob3 only the open reading frame was amplified (coordinates 135500 to 137158). The stop codons were excluded to allow subsequent cloning as $\mathrm{N}$-terminal fusion constructs. The PCR products were cloned into the previously prepared C-terminal tag vectors as depicted in table 2.10. The resulting plasmids were checked by analytical digests and sequencing of the whole ORF including the expression tag.

Table 2.10: Cloning strategy for the parental Spt16 and Pob3 plasmids - The open reading frames of Spt16 and Pob3 were cloned as N-terminal tag fusion proteins in yeast expression plasmids. Pob3 expression is controlled by the GALS promoter whereas Spt16 contains its endogenous promoter (500 bp upstream of the start codon).

\begin{tabular}{|c|c|c|c|c|c|}
\hline ORF & PCR primers & used restriction sites & product size (bp) & $\begin{array}{l}\text { cloned into } \\
\text { (table 2.9. }\end{array}$ & final plasmid \\
\hline Spt16 & \#CHR0150 + \#CHR0151 & SacI and SmaI & 3,647 & $\begin{array}{l}\text { p426 GAL 3HA } \\
\text { p426 GAL 3myc } \\
\text { p426 GAL 6HA } \\
\text { p426 GAL 9myc }\end{array}$ & $\begin{array}{l}\text { p426 E Spt16:3HA } \\
\text { p426 E Spt16:3myc } \\
\text { p426 E Spt16:6HA } \\
\text { p426 E Spt16:9myc }\end{array}$ \\
\hline Pob3 & \#CHR0154 + \#CHR0153 & SpeI and SalI & 1,676 & $\begin{array}{l}\text { p426 GAL 3HA } \\
\text { p426 GAL 9myc }\end{array}$ & $\begin{array}{l}\text { p426 GAL Pob3:3HA } \\
\text { p426 GAL Pob3:9myc }\end{array}$ \\
\hline
\end{tabular}

Cloning of the Pob3 homologue from Chaetomium thermophilum: The CTHT0070340 gene (scaffold: scf7180000011822; sequence position: 2148761..2150788 (- strand), uniprot: G0SHK5_CHATD) was amplified from genomic Chaetomium 
thermophilum DNA using the primer pair \#CHR0223/\#CHR0224. The resulting PCR product (2028 bp) was cloned into the pCDF Duet-1 expression vector using the restriction sites EcoRI and HindIII. Genomic Chaetomium thermophilum DNA was a kind gift from Prof. Ficner group. The cloning was confirmed by analytical restriction digest with the same enzymes. The gene is annotated with 4 introns which were removed manually in a sequential fashion by mutagenesis PCR (table 2.11). The final coding sequence has $1716 \mathrm{bp}$ and is consistent with the ENA (European Nucleotide Archive) sequence EGS17694.1.

Table 2.11: Intron removal of ctPob3 - Table shows the primer pairs for PCR mutagenesis on the pCDFD gctPob3 template for removal of introns. *additional TEV cleavage site between His-tag and ctPob3-CDS was introduced by QC with this pair

\begin{tabular}{cccc}
\hline intron & genomic postion & intron length (bp) & $\begin{array}{c}\text { primer for QC } \\
\text { removal }\end{array}$ \\
\hline 1 & $2,150,777-2,150,636$ & 142 & \#CHR0388 + \#CHR0389 \\
& & & \#CHR0390 + \#CHR0391* \\
2 & $2,150,412-2,150,352$ & 61 & \#CHR0204 + \#CHR0205 \\
3 & $2,150,863-2,150,809$ & 55 & \#CHR0206 + \#CHR0207 \\
4 & $2,149,436-2,149,383$ & 54 & \#CHR0208 + \#CHR0209 \\
\hline
\end{tabular}

The resulting plasmids pCDFD His-ctPob3 and pCDFD His-TEV-ctPob3 were checked by sequencing. Finally, the pCDFD His-TEV-ctPob3 was subcloned into pRSF Duet-1 using EcoRI and HindIII restriction sites and checked by sequencing.

Construction of ctPob3 amber mutants for expression and pBPA incorporation in Escherichia coli: The bacterial expression plasmid pRSF His-TEV-ctPob3 was subjected to Quikchange PCR mutagenesis (section 2.2.1.4). Amber codons were introduced at 10 different positions and checked by sequencing (table 2.12).

Creation of a C-terminal deletion of ctPob3 for protein expression in Escherichia coli: The bacterial expression plasmid pCDFD His-TEV-ctPob3 was subjected to PCR mutagenesis using the Phusion ${ }^{\circledR}$ High-Fidelity DNA Polymerase with the primers \#CHR0625 and \#CHR0625 (section 2.2.1.4; table 2.5; extension time: 4 minutes). Plasmids were tested by analytical restriction digest with SalI and HindIII. The final construct, pCDFD His-TEV-ctPob3 $\triangle$ D467-G571, was checked by sequencing for the entire multiple cloning site.

Cloning of yNhp6a for protein expression in Escherichia coli: The ORF of yNhp6a (NHP6A/YPR052C on chromosome ChrXVI from coordinates 665974 to 665693) was amplified from genomic yeast DNA with primers \#CHR0062 and 
Table 2.12: Construction of ctPob3 amber mutants with mutagenesis PCR primers - Table shows the primer pairs for PCR mutagenesis on the pRSF His-TEV-ctPob3 plasmid

\begin{tabular}{cc}
\hline TAG position & primer for PCR mutagenesis \\
\hline V176 & \#CHRH0001 +\#CHRH0002 \\
Y181 & \#CHRH0003 + \#CHRH0004 \\
T186 & \#CHRH0005 + \#CHRH0006 \\
L236 & \#CHRH0007 + \#CHRH0008 \\
Y260 & \#CHRH0009 + \#CHRH0010 \\
D280 & \#CHRH0011 + \#CHRH0012 \\
D310 & \#CHRH0013 + \#CHRH0014 \\
S482 & \#CHRH0015 + \#CHRH0016 \\
S496 & \#CHRH0017 + \#CHRH0018 \\
Y520 & \#CHRH0019 + \#CHRH0020 \\
\hline
\end{tabular}

\#CHR0063 (amplicon length: $321 \mathrm{bp)}$ ). The forward primer contains a TEV-protease cleavage site. Furthermore, the start codon (ATG) was deleted. The PCR product was cloned into bacterial expression vector pCDFDuet ${ }^{\mathrm{TM}}-1$ using BamHI and XhoI restriction sites. The final construct, pCDFD His-TEV-yNhp6a, was checked by sequencing for the entire multiple cloning site.

NLS constructs for GFP localization studies: The Pob3 ORF was subcloned from plasmid p426 GAL Pob3:3HA into the pUG35 pMET yEGFP3 plasmid using SpeI and SalI restriction sites. The resulting plasmid pUG35 pMET Pob3:yEGFP3 contains the Pob3 sequence as a N-terminal fusion to the yEGFP3 protein. The cloning sites were checked by sequencing. Several deletion mutants were prepared by Quikchange mutagenesis PCR using the Phusion ${ }^{\circledR}$ High-Fidelity DNA Polymerase (mutants: table 2.13; for PCR: section 2.2.1.4; table 2.5; extension time: 4 minutes). The final constructs were checked by sequencing for the entire expression cassette.

Table 2.13: NLS constructs for in vivo microscopy analysis - constructs were prepared by Quikchange mutagenesis PCR on the pUG35 pMET Pob3:yEGFP3 plasmid

\begin{tabular}{|c|c|c|}
\hline $\begin{array}{l}\text { Pob3 modifications } \\
\text { modifications }\end{array}$ & $\begin{array}{c}\text { primer pair } \\
\text { for QC mutagenesis }\end{array}$ & final constructs \\
\hline$\Delta 458-552$ & \#CHR0613 + \#CHR0614 & pUG35 pMET Pob3 4 458-552:yEGFP3 \\
\hline$\Delta 544-552$ & \#CHR0607 + \#CHR0608 & pUG35 pMET Pob3 $\triangle 544-552: y E G F P 3$ \\
\hline K547M & \#CHR0609 + \#CHR0610 & pUG35 pMET Pob3K547M:yEGFP3 \\
\hline$\Delta 458-543$ & \#CHR0617 + \#CHR0618 & pUG35 pMET Pob3 $\triangle 458-543: y E G F P 3$ \\
\hline
\end{tabular}

Cloning of Pob3 integration constructs: The terminal Pob3 region (amino acid P305 to E552) was amplified from the wild type pUG35 pMET Pob3:yEGFP3 or the 
C-terminal modified pUG35 pMET Pob3 $\triangle 458-543$ :yEGFP3 plasmid. The directionality of the cloning was determined by using two different primer pairs. The pair \#CHR0048/\#CHR0628 results in a PCR fragment with an N-terminal BamHI and C-terminal XhoI restriction site. Insertion of this fragment in the pRS 306 plasmid results in the a construct where the URA3 auxotrophy marker is oriented towards the N-terminal part of the cloned Pob3 region. In contrast, the pair \#CHR0627/\#CHR0629 results in a PCR fragment with an N-terminal XhoI and C-terminal BamHI restriction site. Insertion of this fragment in the pRS 306 plasmid results in the a construct where the URA3 auxotrophy marker is oriented towards the C-terminal part of the cloned Pob3 region. Therefore, the "URA position" was introduced as an indicator for the direction of the cloned Pob3 region. For clarification see figure 2.2. Using the pUG35 pMET Pob3:yEGFP3 plasmid as template resulted in plasmids
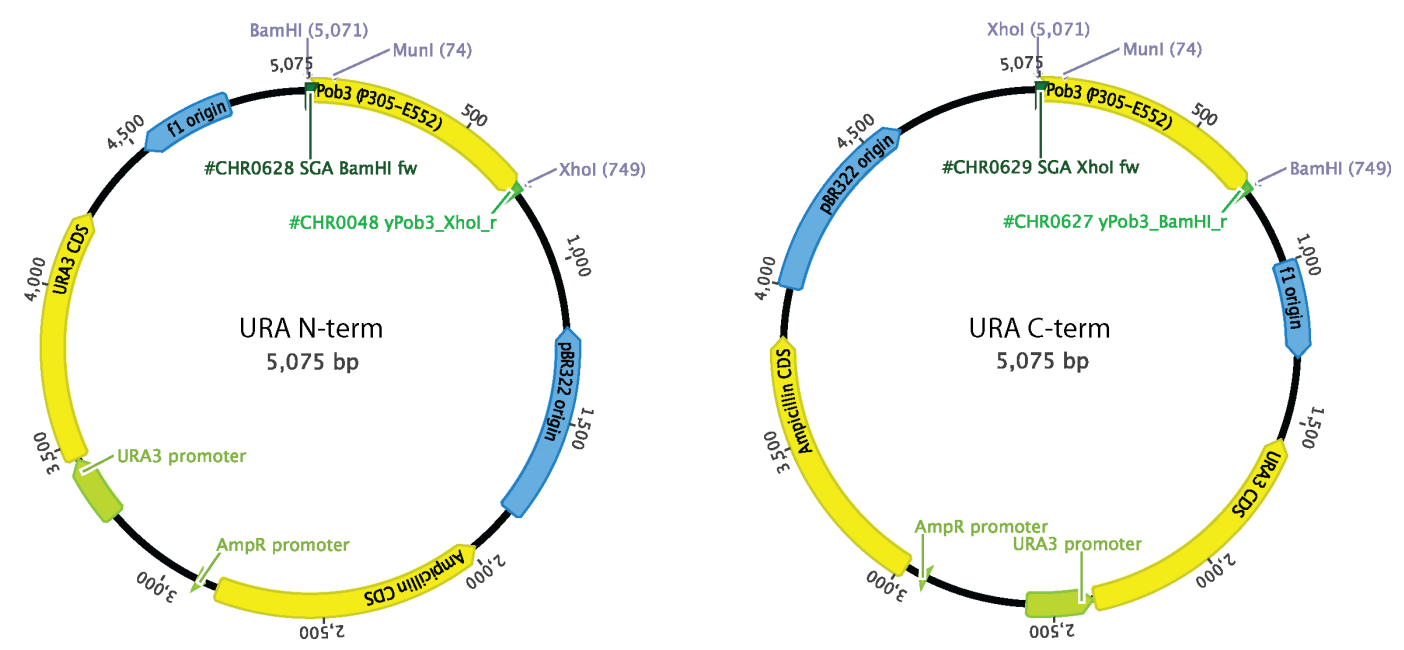

Figure 2.2: Directional cloning of the terminal Pob3 region (amino acid P305 to E552) - Left: Using the primers \#CHR0048/\#CHR0628 and cloning in pRS306 with BamHI and XhoI results in N-terminal position of the URA marker. Right: Using the primers \#CHR0627/\#CHR0629 and cloning in pRS306 with BamHI and XhoI results in C-terminal position of the URA marker; MunI site for linearisation is indicated.

pRS 306 Pob3P305-E552 ( $\mathrm{N}$ and $\mathrm{C}$ ) containing the full-length region P305-E552. In contrast, using pUG35 pMET Pob3 $\triangle 458-543$ :yEGFP3 resulted in plasmids pRS 306 Pob3P305-E552 $\Delta 458-543(\mathrm{~N}$ and $\mathrm{C}$ ) carrying the $\Delta 458-543$ deletion. For the creation of $\Delta$ S491-E543 and $\Delta$ A501-E543 constructs, the pRS 306 Pob3P305-E552 wild type plasmids ( $\mathrm{N}$ and $\mathrm{C}$ ) were subjected to mutagenesis PCR (table 2.14). All constructs were checked by sequencing. 
Table 2.14: Quikchange mutagenesis strategy for $\Delta$ S491-E543 and $\Delta$ A501-E543 constructs - constructs were prepared by Quikchange mutagenesis PCR of the pRS 306 Pob3P305-E552 plasmid with indicated primer pairs

\begin{tabular}{lcc}
\hline $\begin{array}{l}\text { Pob3 } \\
\text { modifications }\end{array}$ & $\begin{array}{c}\text { primer pair } \\
\text { for QC mutagenesis }\end{array}$ & URA position \\
\hline$\triangle$ S491-E543 & \#CHR0877 + \#CHR0878 & $\mathrm{N}$ and C \\
$\triangle \mathrm{A} 501-\mathrm{E} 543$ & \#CHR0875 + \#CHR0876 & $\mathrm{N}$ and C \\
\hline
\end{tabular}

\subsubsection{Protein methods}

\subsubsection{TCA precipitation}

Whole cell extracts were prepared by $\mathrm{NaOH} / \mathrm{TCA}$ method (adapted from Yaffe \&

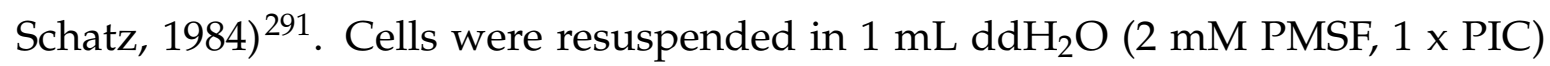
and supplemented with $150 \mu \mathrm{L} 2 \mathrm{M} \mathrm{NaOH}$ and $12 \mu \mathrm{L} \beta$-Mercaptoethanol. The suspension was incubated for 10 minutes on ice. Precipitation was performed by adding $160 \mu \mathrm{L} 50 \%$ (w/v) TCA for 20 minutes on ice. Proteins were collected by centrifugation at $13.000 \mathrm{rpm}$ in a table-top centrifuge $\left(4^{\circ} \mathrm{C}\right)$ for 2 minutes and the pellet was washed once with ice-cold acetone. The supernatant was discarded and the pellet was left to be air-dried. Subsequently, the pellet was resuspended in $75 \mu \mathrm{L}$ 1 x sample buffer and boiled for 10 minutes at $95^{\circ} \mathrm{C}$. The samples were clarified by centrifugation (13.000 rpm at RT) and ready for further analysis by SDS-PAGE or western blotting.

\subsubsection{SDS Polyacrylamide electrophoresis of proteins}

SDS-PAGE was used to separate mixture of proteins based on their molecular weight. In this study, three different types of SDS-PAGE gels were used. First, the common discontinuous SDS-PAGE with a Tris-Glycine buffer system 292 . The composition of the prepared polyacrylamide gels is shown in table 2.15. These gels consist of two layers, including the lower acrylamide percentage stacking gel on top of the higher acrylamide percentage resolving gel. Samples were mixed with 4 x sample buffer and usually boiled at $95{ }^{\circ} \mathrm{C}$ for 10 minutes. Debris was removed by centrifugation at $13.000 \mathrm{rpm}$ in a table-top centrifuge for 2 minutes and the supernatant was loaded. The gels were run in 1 x Tris-Glycine buffer. Electrophoresis was performed at constant current of $45 \mathrm{~mA}$ per gel. The duration of electrophoresis depended on the desired separation range (usually 45-90 minutes).

The second type of SDS-PAGE was performed with commercially available $4-12 \%$ NuPage ${ }^{\circledR}$ Bis-Tris (BT) gels accordingly to manufacturer's instructions. These gra- 
Table 2.15: SDS-PAGE composition - components with stock solutions; amounts are given as final concentrations; gel solutions were prepared with $\mathrm{ddH}_{2} \mathrm{O}$

\begin{tabular}{lclc}
\hline component & stacking gel & component & resolving gel \\
\hline $0.625 \mathrm{M}$ Tris- $\mathrm{HCl} \mathrm{pH} 6.8$ & $0.125 \mathrm{M}$ & $1.5 \mathrm{M}$ Tris- $\mathrm{HCl} \mathrm{pH} 8.8$ & $0.375 \mathrm{M}$ \\
$10 \% \mathrm{SDS}$ & $0.1 \%$ & $10 \% \mathrm{SDS}$ & $0.1 \%$ \\
Rotiphorese $37.5: 1(30 \%)$ & $4 \%$ & Rotiphorese $37.5: 1(30 \%)$ & $12 \% 15 \%$ \\
TEMED & $0.12 \%$ & TEMED & $0.12 \%$ \\
$10 \%$ APS & $0.05 \%$ & $10 \%$ APS & $0.1 \%$ \\
\hline
\end{tabular}

dient gels were commonly used for the analysis of Pob3:9myc crosslink samples. Electrophoresis was performed in 1 x MOPS buffer at 180 volt constant for 90 minutes. The last type of SDS-PAGE was performed with commercially available $3-8 \%$ NuPage ${ }^{\circledR}$ Tris-Acetate (TA) gels accordingly to manufacturer's instructions. These gradient gels were commonly used for the analysis of Spt16:9myc crosslink samples. Electrophoresis was usually performed in $1 \times$ Tris-Acetate buffer at 150 volt constant for $90-120$ minutes. For the NuPage ${ }^{\circledR}$ gels a specialized $4 x$ Loading buffer for NuPage ${ }^{\circledR}$ gels was used.

After electrophoresis, the gel was either stained with a one-step Coomassie-based stain (InstantBlue ${ }^{\mathrm{TM}}$ ) or subjected to western blot 2.2.2.3).

\subsubsection{Western blot and detection}

Western blot is a technique where proteins are transferred from a SDS-PAGE gel onto a membrane and their subsequent detection with specific antibodies. The specific antigen-antibody binding can be detected with various methods. All primary and secondary antibodies used in this thesis, including their working concentrations are shown in table 2.2 .

In this study, two different PVDF membranes were used: Amersham Hybond ${ }^{\mathrm{TM}}$ LFP 0.2 PVDF and Immobilon ${ }^{\circledR}$-P PVDF membrane. The LFP (Low-fluorescent PVDF) was used for subsequent detection with fluorescent secondary antibodies whereas the Immobilon ${ }^{\circledR}$-P PVDF membrane was used for subsequent detection with HRP-coupled secondary antibodies.

Proteins were transferred from either a Tris-Glycine SDS-PAGE gel with $1 \times$ Transfer buffer (25 mM Tris Base, 192 mM Glycine, $0.1 \%$ SDS, $20 \%$ (v/v) Methanol) or from NuPage ${ }^{\circledR}$ Bis-Tris (BT) or Tris-acetate (TA) gels with $1 \times$ Transfer buffer for NuPage ${ }^{\circledR}$ PAGE (25 mM Bicine, 25 mM Bis-Tris, 1.05 mM EDTA, 1.3 mM Sodium Bisulfite, $\mathrm{pH}$ 7.2). Prior to transfer, the membranes were activated in $100 \%$ methanol for 2 minutes followed by incubation in $\mathrm{ddH}_{2} \mathrm{O}$ for 5 minutes. The wet-transfer was done 
after a standard protocol at low voltages (10-20 volt) at $4^{\circ} \mathrm{C}$ overnight. The blotted Immobilon ${ }^{\circledR}$-P PVDF membrane was stained with $0.5 \%$ Ponceaus $S$ solution (in $5 \%(w / v)$ TCA) whereas the LFP membrane was left untreated. The Ponceau S stained membrane was washed with TBS/PBS until the stain was removed. The following paragraph describes the standard procedure whereas the blocking agent (BSA or MLK) as well as blotting buffer (PBS or TBS) changed upon the combination of primary and secondary antibody (table 2.2 for details).

The membrane was washed with PBS/TBS once. Blocking in BSA/MLK was done for 1 to 3 hours at room temperature. The primary-antibody incubation was typically performed overnight at $4{ }^{\circ} \mathrm{C}$ with shaking. In other cases, the primary incubation was done for 2 hours at room temperature. The antibody was recovered and reused several times (stored at $-20^{\circ} \mathrm{C}$ with a final concentration of $0.02 \%(\mathrm{v} / \mathrm{v})$ sodium azide). The membrane was washed with PBS/TBS twice for 10 minutes each. The secondary-antibody incubation was done usually for 1 hour at room temperature with shaking.

For chemiluminescent detection the secondary antibodies $\alpha$-mouse-IgG-HRP or $\alpha$-rabbit-IgG-HRP were used. After incubation, the blot was washed 4 times briefly and 2 times for 10 minutes with TBST/PBST (0.1\% (v/v) Tween 20). The membrane was washed again 2 times with TBS/PBS to remove the Tween. The detection was done with the Amersham ECL Plus ${ }^{\mathrm{TM}}$ or Prime ${ }^{\mathrm{TM}}$ Western Blotting Detection reagent and Amersham Hyperfilm ${ }^{\mathrm{TM}}$ ECL according to manufacturer's instructions.

For fluorescent detection the secondary $\alpha$-mouse-IgG-Cy3 antibody from the Amersham ECL Plex ${ }^{\mathrm{TM}}$ Western Blotting kit was used. The antibody was dissolved in $\mathrm{ddH}_{2} \mathrm{O}$ at a concentration of $1 \mu \mathrm{g} / \mu \mathrm{L}$ and stored in aliquots at $-20^{\circ} \mathrm{C}$. The diluted secondary-antibody solution (1:2.500) was reused several times. After incubation, the blot was washed 4 times briefly and then 2 times for 10 minutes with TBST/PBST $(0.1 \%(v / v)$ Tween 20$)$. The Tween was removed by two additional wash steps with TBS/PBS. For detection, the membrane was dried for at least one hour at $40^{\circ} \mathrm{C}$. Fluorescence detection was performed using the Typhoon 9400 variable mode imager. The Cy3 fluorophore was excited with the green laser (532 nm) and the Cy3 emission filter settings were used for the readout $(580 \mathrm{~nm}$ BP $30 \mathrm{~nm})$. The pixel size, PTM value and sensitivity mode was varied for different experiments.

\subsubsection{Protein expression and purification of yNhp6a from Escherichia coli}

The His-TEV-yNhp6a protein $(13.1 \mathrm{kDa})$ was expressed in E.coli Rosetta $^{\mathrm{TM}}$ 2(DE3) cells and purified by NiNTA affinity chromatography and size exclusion chromatography. 
Expression and lysis of expression cultures: E.coli Rosetta ${ }^{\mathrm{TM}}$ 2(DE3) were transformed with the plasmid pCDFD His-TEV-yNhp6a and spread on agar plates containing appropriate antibiotics (final $35 \mu \mathrm{g} / \mathrm{mL}$ chloramphenicol and $50 \mu \mathrm{g} / \mathrm{mL}$ spectinomycin). A single colony was used to inoculate a $50 \mathrm{~mL}$ preculture with antibiotics. Expression culture (8 L LB medium) was inoculated from the preculture at a final $\mathrm{OD}_{600}$ of 0.05 . Cells were incubated at $37^{\circ} \mathrm{C}$ under shaking conditions (220 rpm). Protein expression was induced with $0.5 \mathrm{mM}$ IPTG at an $\mathrm{OD}_{600}$ of 1.0. Expression was performed at $16^{\circ} \mathrm{C}$ overnight (approximately 16 hours). After expression, cultures had an $\mathrm{OD}_{600}$ of 1.5 and were harvested by centrifugation at $4.800 \mathrm{xg}$ for 15 minutes $\left(4{ }^{\circ} \mathrm{C}\right.$, Sorvall Avanti ${ }^{\circledR}$ J Series, Rotor: JLA-8.1000). Samples for SDS-PAGE analysis were taken before induction and before harvesting the cells. Cells were washed once in $30 \mathrm{~mL}$ PBS. After centrifugation, the pellets were stored at $-20^{\circ} \mathrm{C}$ till further use.

For lysis, a pellet from 4 liters of culture was resuspended in $40 \mathrm{~mL}$ lysis buffer with $2 \mathrm{mM}$ PMSF and 1 x PIC (50 mM Tris-HCl pH 7.2, $150 \mathrm{mM} \mathrm{NaCl}, 20 \mathrm{mM}$ imidazole, $0.5 \mathrm{mM}$ EDTA, $1 \mathrm{mM}$ DTT). After the addition of $0.2 \mathrm{mg} / \mathrm{mL}$ lysozyme and $0.025 \mathrm{mg} / \mathrm{mL}$ DNAseI, the cells were incubates on ice for 20 minutes. Cells were further subjected to sonification two times for 1 minute (Branson Sonifier 450, intensity 8 , duty cycle 60). Insoluble debris was separated form the soluble protein fraction by centrifugation at $18.000 \mathrm{rpm}$ for 25 minutes $\left(4^{\circ} \mathrm{C}, \mathrm{JA}-20\right)$. Samples were taken after sonification, after centrifugation of the soluble supernatant and from the resuspended insoluble pellet.

NiNTA-affinity-purification and size exclusion chromatography: For NiNTAaffinity-purification of the His-TEV-yNhp6a protein, a Qiagen $5 \mathrm{~mL}$ NiNTA Superflow Cartridges with the $\ddot{A} k{ }^{T M}$ prime system was used. The supernatant was loaded to the preequlibrated column using a Superloop at a flow rate of $1.5 \mathrm{~mL} / \mathrm{min}$. Binding and washing was done with the lysis buffer without PMSF and PIC. The column was washed with $50 \mathrm{~mL}$ at $1.5 \mathrm{~mL} / \mathrm{min}$ and then subsequently with another $100 \mathrm{~mL}$ at $5.0 \mathrm{~mL} / \mathrm{min}$ until a UV baseline was reached. Bound proteins were eluted with lysis buffer supplemented with $200 \mathrm{mM}$ imidazole at a flow rate of $1.0 \mathrm{~mL} / \mathrm{min}$. Fractions ( $3 \mathrm{~mL}$ ) were collected and analyzed by SDS-PAGE and Bradford.

Appropriate fractions were pooled and further purified by size exclusion chromatography using a HiLoad ${ }^{\circledR}$ 26/60 Superdex ${ }^{\circledR}$, 75 PG chromatography column. First, the column was equilibrated with gel filtration buffer $(10 \mathrm{mM}$ Tris- $\mathrm{HCl} \mathrm{pH} 7.5$, $150 \mathrm{mM} \mathrm{NaCl}, 1 \mathrm{mM}$ DTT). The chromatography was performed at a flow rate of $1.5 \mathrm{~mL} / \mathrm{min}$ and fraction size was set to $3.5 \mathrm{~mL}$. After gel filtration appropriate fractions were analyzed by SDS-PAGE, pooled and concentrated using a Vivaspin ${ }^{\circledR}$ Centrifugal Concentrator. The purified His-TEV-yNhp6a protein was aliquoted, 
frozen in liquid nitrogen and stored at $-80^{\circ} \mathrm{C}$.

\subsubsection{Protein expression and batch-purification of ctPob3 and ctPob3 $\Delta$ D467-G571 from Escherichia coli}

The His-TEV-ctPob3 protein and the truncated $\triangle \mathrm{D} 467-\mathrm{G} 571$ mutant were expressed in E.coli Rosetta $^{\mathrm{TM}} 2$ (DE3) cells and purified by NiNTA affinity chromatography and size exclusion chromatography.

Expression and lysis of cultures: Transformation (plasmid pCDFD His-TEV-ctPob3 or pCDFD His-TEV-ctPob3 $\triangle$ D467-G571) and preculturing were done as described previously 2.2.2.4. Protein expression was induced with $0.5 \mathrm{mM}$ IPTG at an $\mathrm{OD}_{600}$ of 0.7-0.8 and performed at $37^{\circ} \mathrm{C}$ for 6 hours or at $16^{\circ} \mathrm{C}$ overnight. Cells were harvested by centrifugation (4.800xg for 15 minutes, $4{ }^{\circ} \mathrm{C}$, Sorvall Avanti ${ }^{\circledR}$ J Series, Rotor: JLA-8.1000) and washed once with PBS.

For lysis, a pellet from 2 liters of culture was resuspended in $30 \mathrm{~mL}$ lysis buffer with 2 mM PMSF and $1 \times$ PIC (50 mM Hepes pH 7.2, $500 \mathrm{mM} \mathrm{NaCl}, 20 \mathrm{mM}$ imidazole, $0.5 \mathrm{mM}$ EDTA, $1 \mathrm{mM}$ DTT, $5 \%$ glycerol). After the addition of $0.2 \mathrm{mg} / \mathrm{mL}$ lysozyme and $0.02 \mathrm{mg} / \mathrm{mL}$ DNAseI, the cells were incubated on ice for 20 minutes. Cell disruption was performed by high pressure lysis passing the cell suspension through a fluidizer 3 times at 80 psi following manufacturer's instructions. Insoluble debris was separated form the soluble protein fraction by centrifugation at $30.000 \mathrm{xg}$ for 30 minutes $\left(4^{\circ} \mathrm{C}\right.$, JA-20).

NiNTA-affinity-batch-purification and size exclusion chromatography: The soluble supernatant was incubated with $1 \mathrm{~mL}$ pre equilibrated HisPur ${ }^{\mathrm{TM}} \mathrm{Ni}$-NTA Resin per liter of expression culture. Binding was performed in $50 \mathrm{~mL}$ Falcons for 1.5 hours at $4^{\circ} \mathrm{C}$ on a tube Roller incubator. After binding, the resin was collected by centrifugation at $4000 \mathrm{~g}$ for 3 minutes. The supernatant was discarded and the beads were washed with $50 \mathrm{~mL}$ lysis buffer without PMSF and PIC for 10 minutes under rolling conditions. The washing step was repeated 5 times. Proteins were eluted by adding $10 \mathrm{~mL}$ of lysis buffer supplemented with $200 \mathrm{mM}$ imidazole to the resin for 10 minutes. After centrifugation, the supernatant was filtered using a syringe membrane filter $(0.2 \mu \mathrm{m})$. Samples of the soluble supernatant, the non-bound protein fraction (flow-through) and the elution were analyzed by $12 \%$ SDS-PAGE. The eluate was concentrated to $3 \mathrm{~mL}$ using a Centrifugal Concentrator (30.000 MWCO) and subjected to size exclusion chromatography. The sample was loaded onto a HiLoad ${ }^{\circledR}$ 26/60 Superdex ${ }^{\circledR}, 200$ PG chromatography column which was pre equilibrated 
with sterile and degassed gelfiltration buffer (50 mM Hepes pH 7.2, $250 \mathrm{mM} \mathrm{NaCl}$, $1 \mathrm{mM}$ DTT, $5 \%$ glycerol). The SEC was performed at a flow rate of $1.5 \mathrm{~mL} / \mathrm{min}$ and fraction size was set to $3.0 \mathrm{~mL}$. After gel filtration appropriate fractions were analyzed by SDS-PAGE, pooled and concentrated using a Centrifugal Concentrator. The purified His-TEV-ctPob3 or $\triangle \mathrm{D} 467-\mathrm{G} 571$ mutant protein was aliquoted, frozen in liquid nitrogen and stored at $-80^{\circ} \mathrm{C}$.

\subsubsection{Protein expression and batch-purification of hlmportin- $\alpha$ from Escherichia coli}

The GST-hImportin- $\alpha \Delta$ IBB protein was expressed in E.coli Rosetta $^{\mathrm{TM}} 2$ (DE3) cells and purified by glutathione-affinity and size exclusion chromatography.

For expression the plasmid pGEX6P1 hImp- $\alpha \Delta \mathrm{IBB}$ was used harboring the ampicillin resistance gene for selection (kind gift from Dr. Achim Dickmanns). Main expression cultures were inoculated with a preculture of a single colony (LB with $35 \mu \mathrm{g} / \mathrm{mL}$ chloramphenicol and $100 \mu \mathrm{g} / \mathrm{mL}$ ampicillin) to achieve a final $\mathrm{OD}_{600}$ 0.1. Protein expression was induced with $0.5 \mathrm{mM}$ IPTG at an $\mathrm{OD}_{600}$ of $0.7-0.8$ and performed at $16^{\circ} \mathrm{C}$ for 6-8 hours. Harvest, lysis and batch purification is described previously in section 2.2.2.5 whereas the pellet was lysed in GSH-lysis buffer (50 mM Hepes $\mathrm{pH} 7.2,400 \mathrm{mM} \mathrm{NaCl}, 1 \mathrm{mM}$ DTT) and purified using $1 \mathrm{~mL}$ pre equilibrated Glutathione Sepharose $4 \mathrm{~B}$ per liter of expression culture. For elution the GSH lysis buffer was supplemented with $30 \mathrm{mM}$ glutathione. The $\mathrm{pH}$ of the elution buffer was readjusted to $\mathrm{pH}$ 7.2. Subsequent size exclusion chromatography was performed as described in section 2.2.2.5. The purified GST-hsImportin $\alpha$ protein was aliquoted, frozen in liquid nitrogen and stored at $-80^{\circ} \mathrm{C}(50 \mathrm{mM}$ Hepes $\mathrm{pH} 7.2$, $250 \mathrm{mM} \mathrm{NaCl}, 1 \mathrm{mM}$ DTT, 5 \% glycerol).

\subsubsection{Protein expression and batch-purification of ctPob3 pBPA mutants from Escherichia coli}

E.coli BL21 cells were transformed with plasmid pSUP $\mathrm{pBPA}^{233}$ and with plasmids containing ctPob3 ORF with different amber mutations (pRSF His-TEV-ctPob3TAG, table 2.12). Main expression cultures were inoculated with a preculture from a single colony (LB with $35 \mu \mathrm{g} / \mathrm{mL}$ chloramphenicol and $50 \mu \mathrm{g} / \mathrm{mL}$ kanamycin) to achieve a final $\mathrm{OD}_{600}$ of 0.15 . The unnatural amino acid pBPA was added to a final concentration of $1 \mathrm{mM}$ from a $100 \mathrm{mM}$ pBPA stock. Protein expression was induced with $1 \mathrm{mM}$ IPTG at an $\mathrm{OD}_{600}$ of $0.6-0.8$ and performed at $37^{\circ} \mathrm{C}$ for 3 hours. Cells were harvested by centrifugation and lysed by sonification as described in section 2.2.2.4. After removal of the insoluble debris, the soluble supernatant was subjected 
to NiNTA affinity batch purification (2.2.2.5). After elution from the NiNTA resin, proteins were aliquoted, frozen in liquid nitrogen and stored at $-80^{\circ} \mathrm{C}$.

\subsubsection{Immunoprecipitation of Pob3:9myc crosslink samples}

Yeast cells expressing plasmid borne Pob3:9myc with incorporated pBPA in response to an amber codon were subjected to crosslinking as described in 2.2.4.5. Crosslink samples or non-UV treated control samples (360 OD 600 from $450 \mathrm{~mL}$ culture) were resuspended in $10 \mathrm{~mL}$ of $1 \times$ RIPA buffer with $2 \mathrm{mM}$ PMSF, $1 \times$ PIC and $200 \mu \mathrm{L}$ PIC-P9599 (1 x RIPA: 15 mM sodium phosphate (pH 7.2), 1 \% (v/v) Trition X-100, $1 \%(\mathrm{w} / \mathrm{v})$ sodium deoxycholate, $0.1 \%$ (w/v) SDS, $150 \mathrm{mM} \mathrm{NaCl}, 10 \mathrm{mM}$ EDTA). Cells were dropped into liquid nitrogen and collected. The frozen pellets were ground in a Ultra Centrifugal Mill ZM 200 from Retsch. The powder was collected in a glass beaker and kept on ice.

$40 \mu \mathrm{L}$ Protein G Dynabeads ${ }^{\mathrm{TM}}$ were incubated with $5 \mu \mathrm{g}$ anti-myc-antibody in a $200 \mu \mathrm{L}$ coupling buffer for 45 minutes (PBS supplemented with $5 \mathrm{mg} / \mathrm{mL}$ BSA). After coupling, beads were washed once with PBS/BSA and resuspended in $300 \mu \mathrm{L}$ PBS. The thawed lysate $(6 \mathrm{~mL})$ was incubated with $40 \mu \mathrm{L}$ anti-myc-coupled protein $\mathrm{G}$ Dynabeads ${ }^{\mathrm{TM}}$ with rotation at $4^{\circ} \mathrm{C}$ for 2 hours. The Dynabeads were collected and a sample was taken form the non-bound supernatant (sample FT). The beads were washed once with $1 \mathrm{~mL}$ of $1 \times$ RIPA with $2 \mathrm{mM}$ PMSF and $1 \times \mathrm{PIC}$ at $4^{\circ} \mathrm{C}$ for 10 minutes. The washing steps were repeated 6 times with $500 \mu \mathrm{L} 1 \times$ RIPA for 5 minutes each. A sample was taken from the final wash step. After washing, the elution was performed by boiling the beads in $27 \mu \mathrm{L}$ RIPA/ 1 X Sample Buffer at $95^{\circ} \mathrm{C}$ for 10 minutes. Samples were analyzed by SDS-PAGE and western blot.

\subsubsection{Chromatin methods}

\subsubsection{Reconstitution of Xenopus laevis histone dimers, tetramers and octamers}

Xenopus laevis histone proteins were expressed and purified following the procedure described by Karolin Luger et al. .80181. For reconstitutions, lyophilised histone proteins were resuspended in unfolding buffer (20 mM Tris- $\mathrm{HCl}$ pH 7.5, $6 \mathrm{M}$ Guanidinium hydrochloride (Fluka ${ }^{\circledR}$ Analytical), 5 mM DTT). Proteins were dissolved in a thermomixer at room temperature for approximately 15 minutes. Protein concentration was determined by UV absorption at $276 \mathrm{~nm}$ (specific molar extinction coefficient: table 2.16.

Histone particles were reconstituted by mixing equimolar amounts of $\mathrm{H} 2 \mathrm{~A} / \mathrm{H} 2 \mathrm{~B}$, 
Table 2.16: Molecular weights and molar extinction coefficients $(\epsilon)$ for full-length Xenopus laevis histone proteins (taken from ${ }^{81}$ )

\begin{tabular}{lcc}
\hline Histone & Molecular weight $(\mathrm{Da})$ & $\epsilon(\mathrm{cm} / \mathrm{M}), 276 \mathrm{~nm}$ \\
\hline $\mathrm{H} 2 \mathrm{~A}$ & 13,960 & 4050 \\
$\mathrm{H} 2 \mathrm{~B}$ & 13,774 & 6070 \\
$\mathrm{H} 3$ & 15,273 & 4040 \\
$\mathrm{H} 4$ & 11,236 & 5400 \\
\hline
\end{tabular}

$\mathrm{H} 3 / \mathrm{H} 4$ or all four core histones preparing histone dimers, tetramers or histone octamers respectively. Mixtures were dialyzed against at least four changes of the 100 -fold volume of refolding buffer overnight at $4^{\circ} \mathrm{C}(2 \mathrm{M} \mathrm{NaCl}, 10 \mathrm{mM}$ Tris- $\mathrm{HCl}$ pH 7.5, 1 mM EDTA, 5 mM $\beta$ ME; membrane: Spectra/Por ${ }^{\circledR}$ MWCO 6,000-8,000). Reconstitutions were concentrated to approximately $500 \mu \mathrm{L}$ and filtered sterile by centrifugation using $\operatorname{Costar}{ }^{\circledR}$ Spin- $X^{\circledR}$ Centrifuge Tube at $4^{\circ} \mathrm{C}$. The samples were loaded onto a pre equilibrated Superdex ${ }^{\mathrm{TM}} 20010 / 300$ GL column for purification of the reconstituted histone particles at $4^{\circ} \mathrm{C}(10 \mathrm{mM}$ Tris- $\mathrm{HCl} \mathrm{pH}$ 7.5, $2 \mathrm{M} \mathrm{NaCl}$, $1 \mathrm{mM}$ EDTA, $5 \mathrm{mM} \beta \mathrm{ME}$, sterile-filtered and degassed). Fractions were collected $(0.5 \mathrm{~mL})$ and analyzed by $15 \%$ SDS-PAGE. Fractions containing the desired histone species (dimers, tetramers or octamers) were pooled and the protein concentration was determined by Bradford (BSA standard) and UV spectroscopy at $276 \mathrm{~nm}$.

\section{Mononucleosome reconstitution by salt dialysis}

The 187 bp long DNA template contains the 147 bp long non-natural '601' positioning sequence ${ }^{2931294}$ (generous gift from the group of Dr. Wolfgang Fischle). Nucleosome were reconstituted by slow dialysis form high salt $(2 \mathrm{M} \mathrm{NaCl})$ to low salt conditions $(20 \mathrm{mM} \mathrm{NaCl})$.

Histone octamers were titrated against the DNA template in octamer:DNA ratios 0.5 to 1.2 . Typically, $80 \mathrm{pmol}$ of nucleosomes were reconstituted in a final volume of $100 \mu \mathrm{L}$. The reactions were set up in Slide-A-Lyzer MINI Dialysis Devices (7K MWCO) at $4^{\circ} \mathrm{C}$. First RB high buffer (10 mM Tris- $\mathrm{HCl} \mathrm{pH}$ 7.5, $2 \mathrm{M} \mathrm{NaCl}, 1 \mathrm{mM}$ EDTA, $1 \mathrm{mM}$ DTT) was mixed with the DNA template which was diluted with a $5 \mathrm{M}$ $\mathrm{NaCl}$-solution in $\mathrm{ddH}_{2} \mathrm{O}$ to a final concentration of $2 \mathrm{M} \mathrm{NaCl}$. The varying amounts of histone octamers were added last and the reaction was mixed by pipetting. The dialysis units were capped, placed in a float-rack and transferred to a glass beaker with $400 \mathrm{~mL} \mathrm{RB}$ high buffer at $4^{\circ} \mathrm{C}$. A dialysis apparatus was set up as described by Luger et al., 1999. Dialysis was performed by adding RB-low buffer drop wise using a peristaltic pump at a flow rate of approximately $1 \mathrm{~mL} / \mathrm{min}(10 \mathrm{mM}$ Tris- $\mathrm{HCl} \mathrm{pH} \mathrm{7.5,}$ $20 \mathrm{mM} \mathrm{NaCl}, 1 \mathrm{mM}$ EDTA, $1 \mathrm{mM}$ DTT). The excessive amount of buffer was pumped 
out at a higher flow rate. Dialysis was carried out for approximately 24 hours against at least 1.6 L RB-low buffer. Subsequently, samples were analyzed by $4 \%$ native PAGE (as described in 2.2.3.2).

\subsubsection{Native Polyacrylamide electrophoresis and Nhp6a binding assays}

Native PAGE separation was performed under non-denaturing conditions, thereby allowing protein-protein or nucleic acid-protein complexes to remain intact and comigrate. The gel preparation process is similar as described for SDS-PAGE (2.2.2.2) but without the addition of SDS. In this study, native PAGE was used for the analysis of reconstituted mononucleosomes and protein binding assays of nucleosomes to yNhp6a. The gels were prepared after Ruone et al. (2003) $)^{188}$ (table 2.17).

Table 2.17: SDS-PAGE composition - components with stock solutions; amounts are given as final concentrations; gel solutions were prepared with $\mathrm{ddH}_{2} \mathrm{O}$

\begin{tabular}{lcc}
\hline component & final concentration & amount for $\mathbf{4}$ gels (final $\mathbf{6 0} \mathbf{~ m L}$ ) \\
\hline $\mathrm{ddH}_{2} \mathrm{O}$ & & $43 \mathrm{~mL}$ \\
Acrylamid 19:1 (30\%) & $4 \%$ & $8 \mathrm{~mL}$ \\
Glycerol 99.5\% & $5 \%$ & $3 \mathrm{~mL}$ \\
$\mathrm{MgCl}_{2}(1 \mathrm{M})$ & $2 \mathrm{mM}$ & $120 \mu \mathrm{L}$ \\
$\mathrm{TBE}(5 \mathrm{x})$ & $0.5 \mathrm{x}$ & $6 \mathrm{~mL}$ \\
$\mathrm{TEMED}$ & $0.05 \%$ & $32 \mu \mathrm{L}$ \\
$10 \%$ APS & $0.07 \%$ & $400 \mu \mathrm{L}$ \\
\hline
\end{tabular}

Nucleosomes were mixed with glycerol (final concentration $20 \%$ ) and analyzed by $4 \%$ PA gels in 0.5 x TBE. Electrophoresis was performed at 100-150 volt for 1.5 hours. After electrophoresis, the DNA was stained by incubating the gel in a GelRed Nucleic Acid Stain (1:10.000) bath for approximately 20 minutes. Subsequently, the gel was analyzed with the Gel Doc ${ }^{\mathrm{TM}} 2000$ from Bio-Rad.

For binding reactions of nucleosomes to yNhp6a protein, nucleosomes were mixed with 5x BM buffer and incubated at $30^{\circ} \mathrm{C}$ for 30 minutes (5x BM: $100 \mathrm{mM}$ Hepes pH 7.6, $600 \mathrm{mM} \mathrm{NaCl}, 1 \mathrm{mM} \beta \mathrm{ME}, 4.5 \mathrm{mg} / \mathrm{mL}$ BSA, 60 \% Sucrose, $10 \%$ glycerol). Electrophoresis and imaging was performed as described above.

\subsubsection{Yeast methods and crosslinking}

\subsubsection{Yeast culturing}

Yeast strains lacking plasmids were grown and maintained on standard YPD medium. Auxotrophic yeast strains carrying plasmids were cultured in standard SD medium 
(1.7 g/L BD Difco ${ }^{\circledR}$ Yeast Nitrogen Base w/o Amino Acids \& Ammonium Sulfate, $5 \mathrm{~g} / \mathrm{L}$ Ammonium sulfate, $2 \mathrm{~g} / \mathrm{L}$ amino acid drop out mixture, $\mathrm{pH}$ adjustment with $800 \mu \mathrm{L} 4 \mathrm{M} \mathrm{NaOH}$ per liter medium). Prior to sterilization of the SD medium, it was supplemented with the required amino acid(s) (uracil, tryptophan, histidine and leucine) allowing propagation and maintenance of plasmid(s) with auxotrophy markers. The carbon source varied due to experimental setup (final concentrations: $2 \%(\mathrm{w} / \mathrm{v})$ glucose, $2 \% \mathrm{w} / \mathrm{v}$ raffinose, $2 \% \mathrm{w} / \mathrm{v}$ galactose, $2 \% \mathrm{w} / \mathrm{v}$ galactose and $1 \%$ $\mathrm{w} / \mathrm{v}$ raffinose). Cells were grown under standard conditions at $30^{\circ} \mathrm{C}$ with $220 \mathrm{rpm}$ shaking. For temperature sensitive strains (pob3-L78R and spt16-ts) altered temperatures were used dependent upon the experimental details. Solid culture plates were prepared with $1.5 \%$ (w/v) agar final. Additives such as pBPA, hydroxyurea (HU) or methyl methanesulfonate (MMS) were added to cooled medium before pouring the plates. For yeast growth sensitivity assays, serial dilutions of yeast cultures in $\mathrm{ddH}_{2} \mathrm{O}$ were performed and samples were spotted on agar plates with a replicator tool.

\subsubsection{Standard yeast genetic manipulation}

Transformations were done using a standard heat shock LiAc method. Yeast cells $\left(50 \mathrm{OD}_{600}\right)$ of a fresh overnight culture was pelleted by centrifugation, washed with $\mathrm{ddH}_{2} \mathrm{O}$ and resuspended in $2 \mathrm{~mL}$ resuspension solution (0.5 x TE / $\left.100 \mathrm{mM} \mathrm{LiOAc}\right)$. The transformation mixture was prepared and incubated for 30 minutes at $30^{\circ} \mathrm{C}$ (table 2.18). Yeast cells were heatshocked for 15 minutes at $42^{\circ} \mathrm{C}$. The cells were then

Table 2.18: Pipetting scheme of a standard yeast transformation - PEG solution: 1 x TE / 100 mM LiOAc / 50 \% (w/v) PEG 3350

\begin{tabular}{lc}
\hline component & amount in $\mu \mathbf{L}$ \\
\hline DNA & $1 \mu \mathrm{g}$ DNA (usually $5 \mu \mathrm{L}$ of a plasmid prep) \\
ssDNA $(10 \mathrm{mg} / \mathrm{mL})$ & 10 \\
yeast cells & $100\left(\sim 3^{*} 10^{7}\right.$ cells $)$ \\
PEG solution & 700 \\
\hline
\end{tabular}

centrifuged and the pellet was washed once with $\mathrm{ddH}_{2} \mathrm{O}$. Cells were collected by centrifugation and the supernatant was discarded. The pellet was resuspended in the residual $\mathrm{ddH}_{2} \mathrm{O}$ and spread on selective $\mathrm{SD}$ medium plates. Plates were incubated at $30^{\circ} \mathrm{C}$ for 3 days.

In addition to standard transformation, frozen competent yeast cells of several strains were prepared as previously described ${ }^{295}$. Preparation and transformation were performed precisely following the published protocol. 


\subsubsection{Subcellular fractionation of yeast cells}

This protocol was adapted from the yeast nuclei isolation protocol from the Grunstein lab (http://www.biolchem.ucla.edu/labs/grunstein/protocols.html). Yeast cells $\left(75 \mathrm{OD}_{600}\right)$ were resuspended in $2 \mathrm{~mL}$ of a buffer containing $50 \mathrm{mM}$ Tris- $\mathrm{HCl}(\mathrm{pH}$ 7.5) and $30 \mathrm{mM}$ DTT. Samples were incubated for 15 minutes at $30^{\circ} \mathrm{C}$ with shaking (850 rpm in a thermomixer). Cells were collected at 1500xg in a tabletop centrifuge and resuspended in $2 \mathrm{~mL}$ YPD/S with $100 \mathrm{U} / \mathrm{mL}$ lyticase (YPD/S: $10 \mathrm{~g} / \mathrm{L}$ yeast extract, $20 \mathrm{~g} / \mathrm{L}$ Bactotryptone, $20 \mathrm{~g} / \mathrm{L}$ glucose, $182.17 \mathrm{~g} / \mathrm{L}$ sorbitol). The mixture was supplemented with 2 x PIC, $1 \mathrm{mM}$ PMSF and $1 \mathrm{mM}$ DTT. Spheroplasting was performed for approximately 40 minutes at $30^{\circ} \mathrm{C}$. Samples were inverted approximately every 10 minutes. The progress of spheroplasting was examined by microscopy in the presence or absence of 0.1 (w/v) SDS. Spheroplasts were collected at 1500xg for 10 minutes and carefully washed once with $2 \mathrm{~mL}$ YPD/S. The following steps were performed at $4^{\circ} \mathrm{C}$. The washing steps were repeated twice with ice-cold YPD/S and finally with $1 \mathrm{~mL} 1 \mathrm{M}$ ice-cold sorbitol. Spheroplasts were collected for 10 minutes at 1500xg and resuspended in $4 \mathrm{~mL}$ Buffer A supplemented with $3 \mathrm{mM}$ DTT, 2 x PIC and $1 \mathrm{mM}$ PMSF (Buffer A: $18 \%(\mathrm{w} / \mathrm{v})$ polysucrose 400, $10 \mathrm{mM}$ Tris-HCl (pH 7.5), $20 \mathrm{mM} \mathrm{KCl}, 5 \mathrm{mM} \mathrm{MgCl}$, $1 \mathrm{mM}$ EDTA, $0.15 \mathrm{mM}$ spermine, $0.5 \mathrm{mM}$ spermidine). Spheroplasts were lysed using a Dounce tissue grinder (working volume $7 \mathrm{~mL}$, pestle B clearance: 0.0008-0.0022 in.). The pestle was moved up and down 20 times. The lysate was transferred to a $15 \mathrm{~mL}$ Falcon tube. Cellular debris was collected by centrifugation for 5 minutes at $4100 \mathrm{xg}$ at $4^{\circ} \mathrm{C}$. The supernatant was transferred to $1.5 \mathrm{~mL}$ tubes and the nuclei collected by centrifugation at $13.000 \mathrm{xg}$ for 30 minutes. The supernatant (containing the cytosolic proteins; approximately $3.5 \mathrm{~mL}$ ) was TCA precipitated with $500 \mu \mathrm{L} 50 \%(\mathrm{w} / \mathrm{v})$ TCA over night at $4^{\circ} \mathrm{C}$. The nuclei were washed in fresh Buffer A and subjected to TCA precipitation as described in section 2.2.2.1.

\subsubsection{Live cell imaging of yeast}

Yeast cells were picked form a freshly grown plate and resuspended in a small amount of $\mathrm{H}_{2} \mathrm{O}(2-5 \mu \mathrm{L})$. Cells were spread onto a microscope slide (Superfrost ${ }^{\mathrm{TM}}$ Microscope Slides from Thermo Scientific). The cover glass was sealed with nail polish and the slides were imaged immediately. For imaging a commercially available modified platform form $3 \mathrm{i}$ was used (Intelligent Imaging Innovations). It contains the Zeiss AxioObserver.Z1 Inverted Microscope, the spinning disk confocal unit (CSU-X1) connected to the QuantEM:512SC EMCCD Camera. Solid state diode lasers (LaserStack $^{\mathrm{TM}}$ ) were used as light source for GFP excitation. All images were taken with the Zeiss Objective Plan-Apochromat 63x/1.40 using immersion oil and were 
processed using Fiji and Photoshop.

\subsubsection{In vivo crosslinking using pBPA in Saccharomyces cerevisiae}

Plasmids containing Pob3 or Spt16 amber mutations were cotransformed into yeast cells with the plasmid pESC pBPA-RS (LEU). The plasmid pESC pBPA-RS harbors the amber suppressor E. coli tyrosyl tRNA $\mathrm{CUA}_{\mathrm{A}}$ and the evolved E. coli tyrosyl-tRNA synthetase (TyrRS) for the incorporation of pBPA. The variant that is used in this thesis resembles the published p-benzoylPheRS-2 variant created by Jason Chin et al. in $2003^{255}$ and contains the following mutations: Y37G, D182G, F183Y and L186M. Yeast cells were cultured in the appropriate SD medium as described in 2.2.4.1. Cells containing Pob3 constructs were precultured in $2 \%$ raffinose whereas cells with Spt16 constructs were precultured in $2 \%$ glucose. For full-length expression of pBPA mutants, cultures were inoculated at an OD600 of 0.2-0.3 in fresh SD medium supplemented with $1 \mathrm{mM}$ pBPA (4-Benzoyl-L-phenylalanine , $\geq 97 \%$ (HPLC), CHEM-IMPEX International Inc.). For Pob3 mutants the SD-medium was supplemented with $2 \%(\mathrm{w} / \mathrm{v})$ galactose. Occasionally, medium contained an additional $1 \%$ raffinose for better growth. Cells were harvested at late exponential phase (approximately $12 \mathrm{OD}_{600}$ ) by centrifugation and resuspended in $100 \mu \mathrm{L}$ of SDmedium. Samples were subjected to $365 \mathrm{~nm}$ UV irradiation on ice (Vilber Lourmat VL-208.BL, 365nm tubes, 2x8W). The distance form the light source to the samples was approximately $5 \mathrm{~cm}$. Exposure times varied during different experimental setups, but typically crosslinking was performed for 7 to $15 \mathrm{~min}$. Samples were either stored at $-20^{\circ} \mathrm{C}$ or subsequently subjected to TCA precipitation and western blot.

Crosslinking assays with 4-Azido-L-phenylalanine (pAzF) were performed in a similar way. For the incorporation of $\mathrm{pAzF}$ in response to an amber codon in Saccharomyces cerevisiae the plasmid pESC pAzF-RS (LEU) was used. The variant which is used in this thesis resembles the published p-azidoPheRS-3 variant created by Jason Chin et al. in $2003^{255}$ and contains the following mutations: Y37L, D182S, F183A, L186A. Yeast cells were cultured in the appropriate SD medium as described in 2.2.4.1 and full-length expression of pAzF mutants was facilitated by supplementing the medium with $2 \mathrm{mM}$ 4-Azido-L-phenylalanine final (4-Azido-L-phenylalanine, $\geq 99 \%$ (HPLC), Chem-Impex International, Inc.). Crosslinking was done as described previously.

Crosslinking cultures were prepared in low-scale using $20 \mathrm{~mL}$ of expression culture in small Erlenmeyer flasks. For high throughput crosslinking, such as the scan of amber mutant libraries of Spt16 and Pob3, precultures and main cultures were set up in 48 well plates (riplate $\mathbb{R}$ SW, PP, $5 \mathrm{ml}$ ) with $3.5 \mathrm{~mL}$ medium per well. 
Expression cultures were supplemented with $1 \mathrm{mM}$ pBPA final concentration. Cells were harvested at late exponential phase by centrifugation for 2 minutes at $805 x \mathrm{x}$. The supernatant was discarded and the entire 48 well plate was subjected to UV

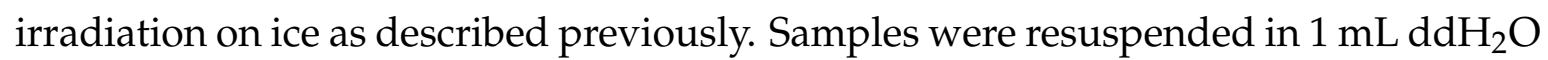
(2 mM PMSF, $1 \times$ PIC), transferred to $1.5 \mathrm{~mL}$ tubes and subjected to TCA precipitation.

\subsubsection{In vitro crosslinking of proteins}

In vitro crosslinking assays were performed by preparing mixtures of pBPA-containing proteins with histone dimers, tetramers or nucleosomes and subjecting them to UV crosslinking. Total protein concentrations were determined with Bradford after a standard protocol (BSA standard). Molar concentrations of proteins and ratios of mixtures were calculated from the determined total protein concentrations and their corresponding molecular weights. Samples were transferred to Eppendorf ${ }^{\circledR}$ UVette ${ }^{\circledR}$ disposable cuvettes and subjected to $365 \mathrm{~nm}$ UV irradiation on ice for 30 to $45 \mathrm{~min}$ utes (Vilber Lourmat VL-208.BL, 365nm tubes, 2x8W). The distance form the light source to the samples was approximately $5 \mathrm{~cm}$. Samples were mixed with $4 x$ Loading buffer and boiled at $95^{\circ} \mathrm{C}$ for 5 minutes. Probes were clarified by centrifugation for 2 minutes at $13.000 \mathrm{rpm}$ in a table-top centrifuge and subjected to SDS-PAGE and western blot. 


\section{Results}

\subsection{In vivo crosslinking studies of FACT in Saccharomyces cerevisiae}

\subsubsection{Detection of plasmid-borne Spt16 and Pob3 harboring different protein tags by western blot}

Detection of an additional plasmid-born copy of the FACT complex subunits is the prerequisite for the in vivo crosslink analysis. Initially the open reading frames of the FACT complex subunits were cloned from genomic yeast DNA and inserted into the yeast expression plasmids with different C-terminal protein tags for western blot detection (myc-tag and HA-tag). The parent plasmids contain either the Spt16-ORF as a fusion construct to different protein tags under the control of the endogenous promoter (p426 E Spt16:myc or HA) or the Pob3-ORF as a fusion to different tags under the control of the GALS promoter (p426 GAL Pob3:myc or HA, see section 2.2.1.12). These parental high-copy ( $2 \mu \mathrm{cron})$ plasmids are the base for amber suppression substitutions introduced by mutagenesis PCR (for details see section 2.2.1.4). Before performing mutagenesis PCR, I tested wild-type BY4741 yeast cells for expression of the parental constructs using SDS-PAGE and western blot (s. figure 3.1).

I successfully detected the expression of proteins in all different epitope-tag combinations. Pob3:HA (figure 3.1 A.) is visualized by two faint bands with a molecular weight of approximately $70 \mathrm{kDa}$ confirming the presence of the Pob3:3HA protein with a calculated molecular weight of $67.4 \mathrm{kDa}$. Samples of cells expressing Pob3:9myc show no signal on the western blot against the HA-protein tag and serve as a negative control. As expected, in the western blot against the myc-epitope, samples of cells that expressed Pob3:9myc show the presence of a protein with the molecular weight of approximately $75 \mathrm{kDa}$. This confirms the expression of the Pob3:9myc fusion protein with a calculated molecular weight of $77.0 \mathrm{kDa}$. Furthermore, Spt16 (figure 3.1 B.) was successfully detected with both HA and myc-tag. Samples of cells expressing Spt16 with a 3HA or 6HA tag show an intense band at 
a molecular weight of approximately $120 \mathrm{kDa}$. This confirms the expression of the Spt16 fusion protein with a 3HA/6HA tag with a calculated (theoretical) molecular weight of $124.5 \mathrm{kDa}$ and $128.2 \mathrm{kDa}$ respectively. Cells expressing Spt16 as a myc-tag fusion construct showed bands of slightly higher molecular weight compared to the HA-tag fusion construct. The calculated molecular weight for Spt16:3/9myc with $125.3 \mathrm{kDa}$ and $134.1 \mathrm{kDa}$ confirms the expression of the fusion tag proteins. Comparing the intensities, longer tags (6HA and 9myc) with a higher number of repeats compared to their shorter versions (3HA and $3 \mathrm{myc}$ ) result in a significant increase of signal intensity. Therefore, I decided to use the longer 9myc tag for further analysis and in vivo crosslinking. It is worth noting that lysate samples for both FACT complex subunits show protein degradation, whereas for Pob3, the degradation was moderate compared to the pattern of Spt16. For further experiments, protease inhibitors (PMSF to $2 \mathrm{mM}$ final and PIC to $1 \mathrm{x}$ final) were added to the resuspended samples before adding SDS-PAGE sample buffer.

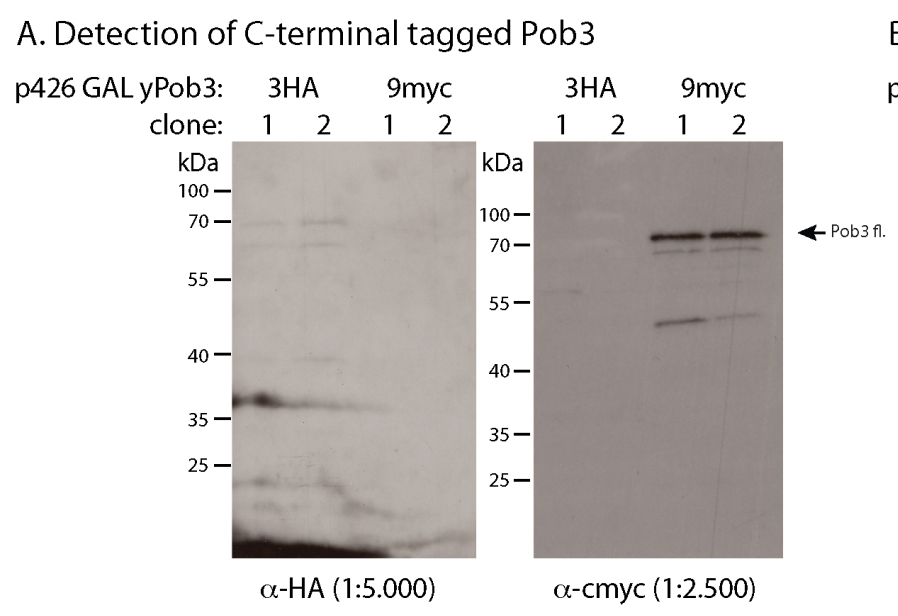

B. Detection of C-terminal tagged Spt16

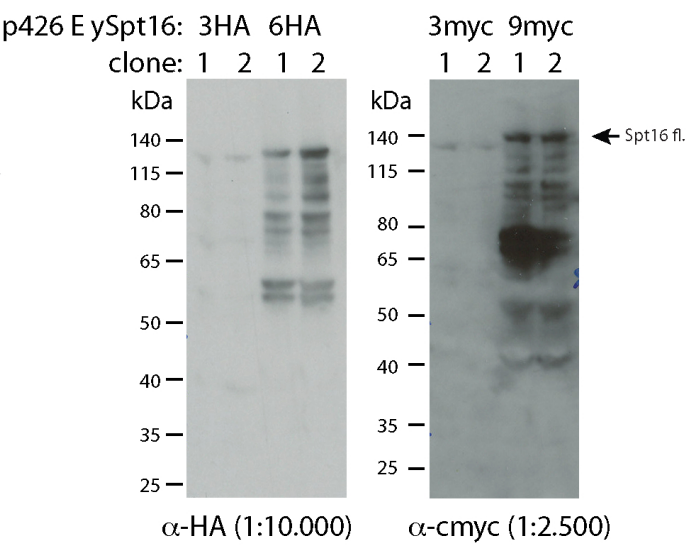

Figure 3.1: Detection of plasmid-borne Spt16 and Pob3 by western blot - A: BY4741-a cells expressing Pob3 with either 3HA or 9myc were grown overnight in SD-URA with $2 \%$ galactose. Samples were prepared by boiling for 5 minutes at $95^{\circ} \mathrm{C}$ and further analyzed on a $12 \%$ SDS-PAGE. Each lane contains $830 \mathrm{mOD}_{600}$ cell lysate. Proteins were transferred onto PVDF membrane and decorated with antibodies against the epitope-tag. B: BY4741-a cells expressing Spt16 with either 3HA or 9myc were grown overnight in SD-URA with $2 \%$ glucose. Samples were prepared and analyzed as shown for yPob3 except for separation of the samples on $4-12 \%$ NuPage ${ }^{\circledR}$ gels in 1x MOPS buffer prior to western blotting.

\subsubsection{In vivo pBPA crosslinking of Pob3 is UV- and position-dependent}

The first pBPA crosslink experiments were performed using 8 different positions on the Pob3 protein (R254, R256, D258, E274, Q310, F315, K322, S500; figure 3.2 B). 


\section{Results}

Plasmids carrying Pob3 with the amber mutations were cotransformed with the pESC pBPA-RS (LEU) plasmid in BY4741 yeast cells. Preculturing was done in raffinose which allowed for subsequent efficient expression of additional plasmid-borne Pob3 after induction with galactose. In general, cells were harvested after they completed at least two cell cycles and hence their $\mathrm{OD}_{600}$ increased fourfold (for the general scheme figure 3.2 A).

\section{A. Experimental setup for Pob3 pBPA crosslinking in yeast}
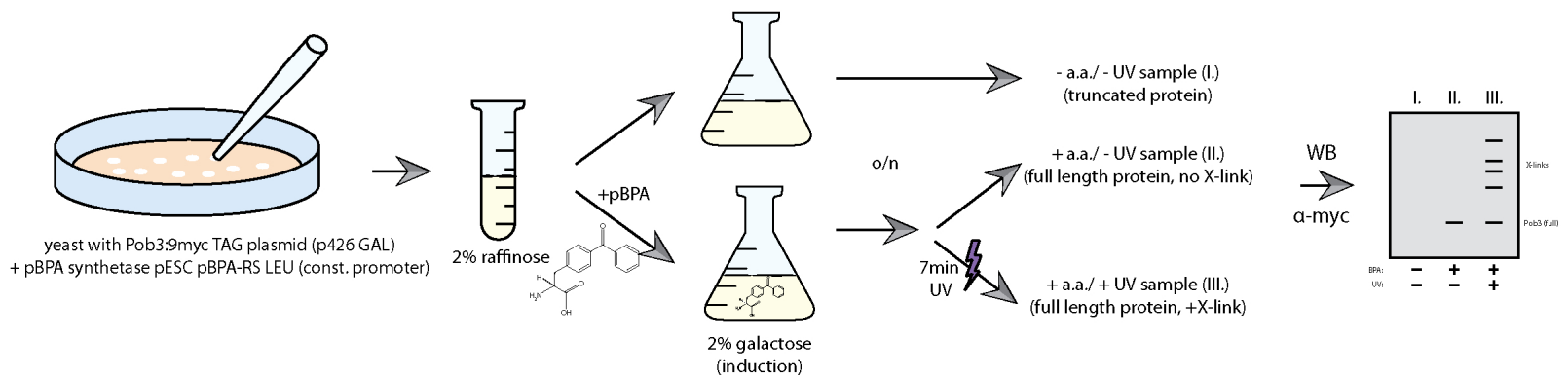

B. Inital crosslinking screen in Pob3-MD
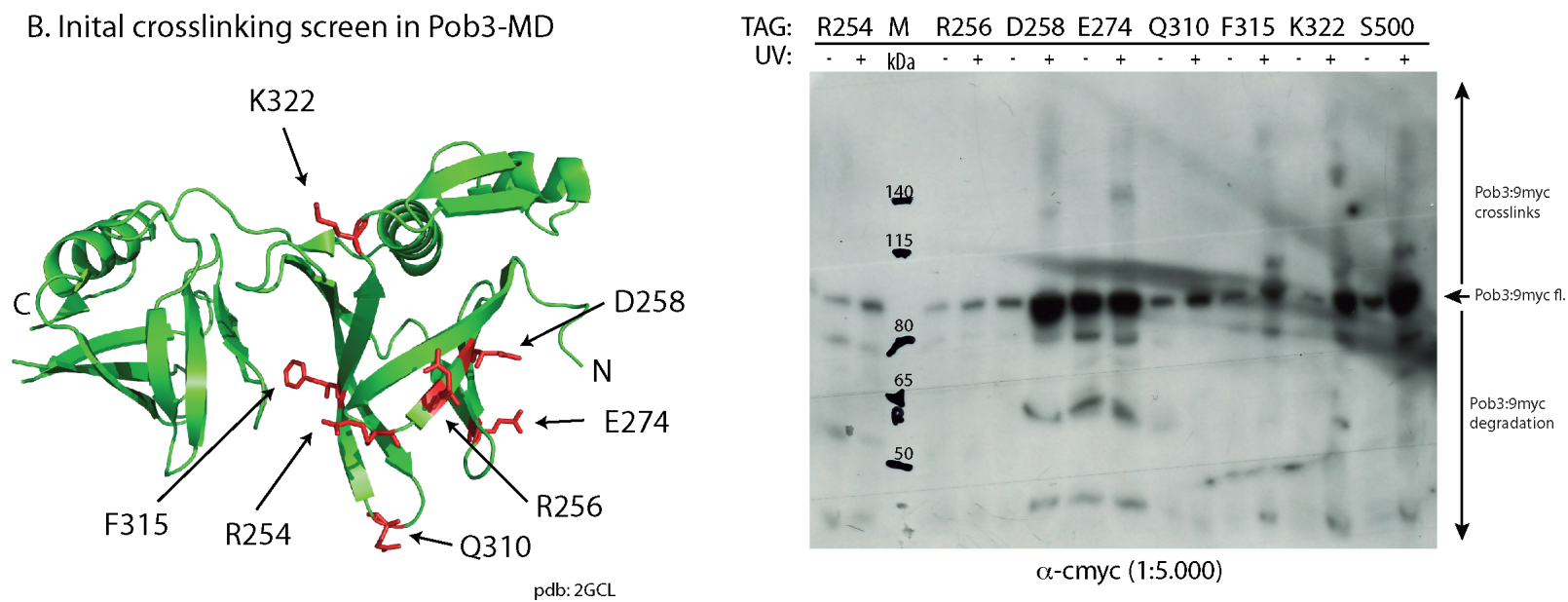

Figure 3.2: Initial pBPA-crosslinking in Pob3-MD reveal UV-dependent and site-specific crosslink formation - A: Yeast cells (BY4741) are cotransfomed with a Pob3:9myc amber plasmid and the pESC pBPA-RS carrying the tools for genetically encoding pBPA. Cells are precultured in raffinose and subsequently diluted to an $\mathrm{OD}_{600}$ of $0.3-0.5$ in medium containing $1 \mathrm{mM}$ pBPA. Cells without pBPA are cultured for control samples. Yeast cells are harbored at an $\mathrm{OD}_{600}$ of approximately 2.0 and a sample of $12 \mathrm{OD}_{600}$ is subjected to $365 \mathrm{~nm}$ light for 7 minutes on ice. Samples are prepared by boiling for 5 minutes at $95^{\circ} \mathrm{C}$ and further analyzed by SDS-PAGE and western blot. B: Samples of an initial pBPA screen at 8 different positions in the Pob3-MD were prepared as described in (A.) and analyzed by western blot (4-12 \% NuPage ${ }^{\circledR}$ gel, $900 \mathrm{mOD}_{600}$ per lane, PVDF membrane, Amersham ECL Plus ${ }^{\mathrm{TM}}$ Western Blotting Detection Reagent)

To characterize the effect of the additional protein expression and induction system, I compared the growth behavior of these yeasts. The average doubling time of wild-type yeast cells is roughly 90-140 minutes ( $\sim 90 \mathrm{~min}$ in YPD and $\sim 140 \mathrm{~min}$ in SD-media) ${ }^{296}$. Apart from the effect of Pob3 expression, yeast cells containing 
the pESC pBPA-RS (LEU) plasmid and an empty p426 GAL plasmid showed an increase in doubling time of $4-5 \mathrm{~h}$ at $30^{\circ} \mathrm{C}$ after induction with $2 \%$ galactose. This could be the result of the carbon source change. Furthermore, for cells expressing the full-length Pob3 from the plasmid p426 GAL Pob3:9myc instead of the empty p426 GAL plasmid, an increase in doubling time to 6-7 h was observed. These results were achieved in the absence of pBPA in the media. The presence of pBPA had only very mild effects on the doubling time, compared to the expression of an additional plasmid-borne and induced copy of Pob3. Despite the increased growth time, no other unusual growth behavior was detected.

The western blot of the in vivo crosslink indicates UV-dependent and site-specific crosslink formation (figure 3.2 B). First, the full-length Pob3:9myc protein is detected at the expected molecular weight of approximately $80 \mathrm{kDa}$ in the presence of pBPA. The slight differences in gel separation are explained by the use of $4-12 \%$ NuPage ${ }^{\circledR}$ gels instead of $12 \%$ SDS-PAGE gels shown in figure 3.1 . There is an obvious formation of UV-dependent crosslinks at the amino acids E274, F315, K322 and S500 indicated by protein signals of higher molecular weight than the full-length Pob3:9myc protein. The crosslinked products show less intensive signals compared to their non-crosslinked Pob3 full-length protein. Furthermore, crosslinked products show different molecular weights at different sites. For instance, the crosslinked product at E274 has a molecular weight of approximately $140 \mathrm{kDa}$ whereas the crosslink at S500 has only a molecular weight of approximately $115 \mathrm{kDa}$. In conclusion, the pBPA in vivo crosslinking technique can be used to analyze interactions in a UV-dependent and site-specific manner. However, conditions, such as sample preparation, crosslink time and blotting conditions, have to be carefully optimized for target proteins.

\subsubsection{Optimization of the crosslinking approach}

In order to improve and standardize the in vivo pBPA crosslinking technique several characterisation experiments were performed. First, I varied the crosslinking time in crosslinking experiments at serine S500 of Pob3. Second, I compared two sample preparation techniques for their application. Finally, I examined the differences between fluorescent and standard chemiluminescent western blot detection and tested crosslinking behavior in protease deficient yeast strains. This thorough characterization is a prerequisite for the subsequent high-throughput crosslink screens. 


\subsubsection{The influence of crosslinking time on the formation of the Pob3 S500 crosslink product}

For the analysis of crosslinking time, experiments for the genetic incorporation of pBPA at serine 500 of Pob3:9myc were performed as described earlier. Samples were placed under UV-light and samples were taken after certain time points of exposure. The lysates were analyzed by western blot and are shown in figure 3.3. Samples of cells which were grown in the absence of pBPA show neglectable amounts of full-length Pob3:9myc protein. As excepted, cells which were grown in the presence of pBPA, show an intensive band for full-length Pob3:9myc protein (last lane). Due to the absence of UV treatment, these sample show no crosslinked products. However, crosslinked products were formed in all samples which were treated with UV-light and contained pBPA. First crosslinked products appeared clearly after one minute of irradiation with increasing intensities observed over thirty minutes. Moreover, a second crosslink product of approximately $140 \mathrm{kDa}$ emerges, only after 5 minutes of irradiation. For subsequent experiments irradiation time was generally set in a range of ten to twenty minutes depending on the experimental setup.

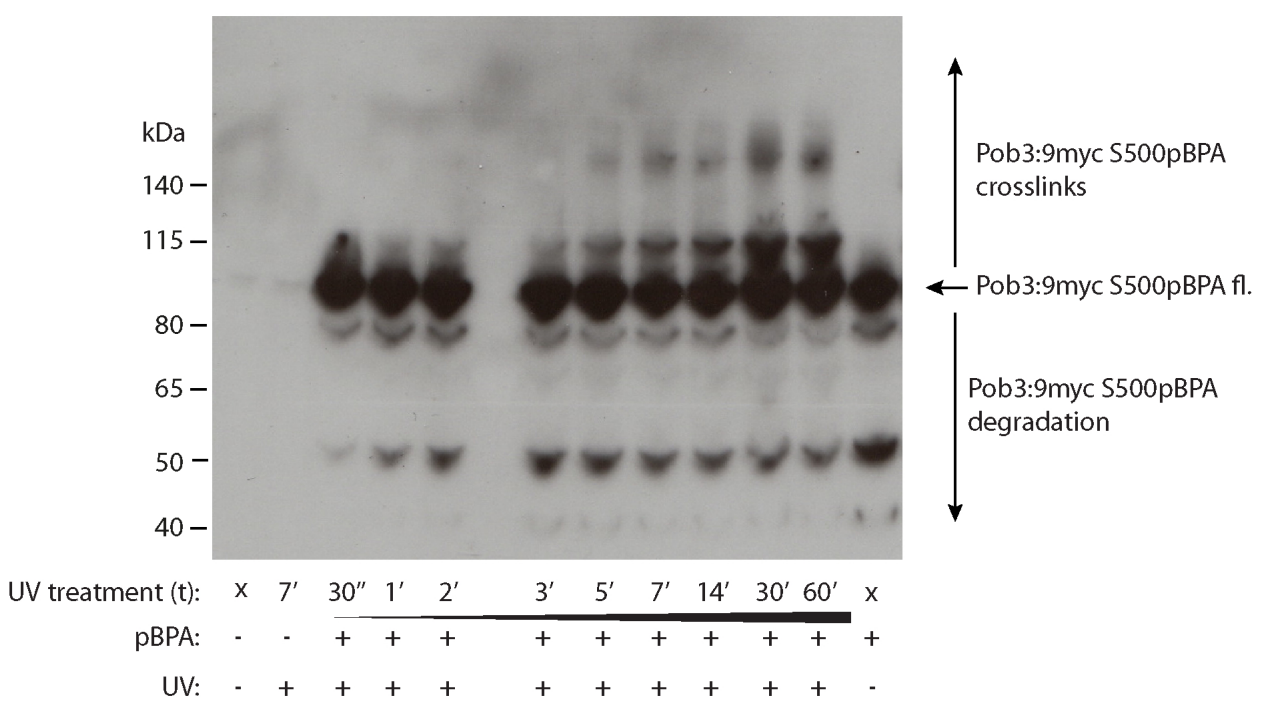

Figure 3.3: Influence of UV exposure time on Pob3 S500 crosslink product formation - In vivo crosslinking experiments were performed with the Pob3:9myc S500TAG construct. Samples were irradiated for different amounts of time ranging from 30 seconds to 1 hour. Samples were prepared by boiling for 5 minutes at $95^{\circ} \mathrm{C}$ and further analysed by SDS-PAGE and western blot (4-12\% NuPage ${ }^{\circledR}$ gel, 1.2 OD $_{600}$ per lane, PVDF membrane, $\alpha$-c-myc, Amersham ECL Plus ${ }^{\mathrm{TM}}$ Western Blotting Detection Reagent). The cell background of the control sample in the last lane was BY4741 with a genomic fusion of GFP to histone H2A. 


\subsubsection{Fluorescent and standard chemiluminescent western blot detection}

For further optimization, I tested different western blot detection systems. Standard chemiluminescent detection with Amersham ${ }^{\mathrm{TM}} \mathrm{ECL}^{\mathrm{TM}}$ Prime Western Blotting Detection Reagent was compared to the western blot detection system using fluorescent secondary antibodies and Typhoon fluorescence scanning (Amersham ${ }^{\mathrm{TM}} \mathrm{ECL}^{\mathrm{TM}}$ Plex CyDye-Conjugated Antibody). In vivo pBPA crosslinking experiments were performed with the Pob3:9myc S500TAG construct and analyzed by SDS-PAGE on a $4-12 \%$ NuPage ${ }^{\circledR}$ gel and western blot (figure 3.4).
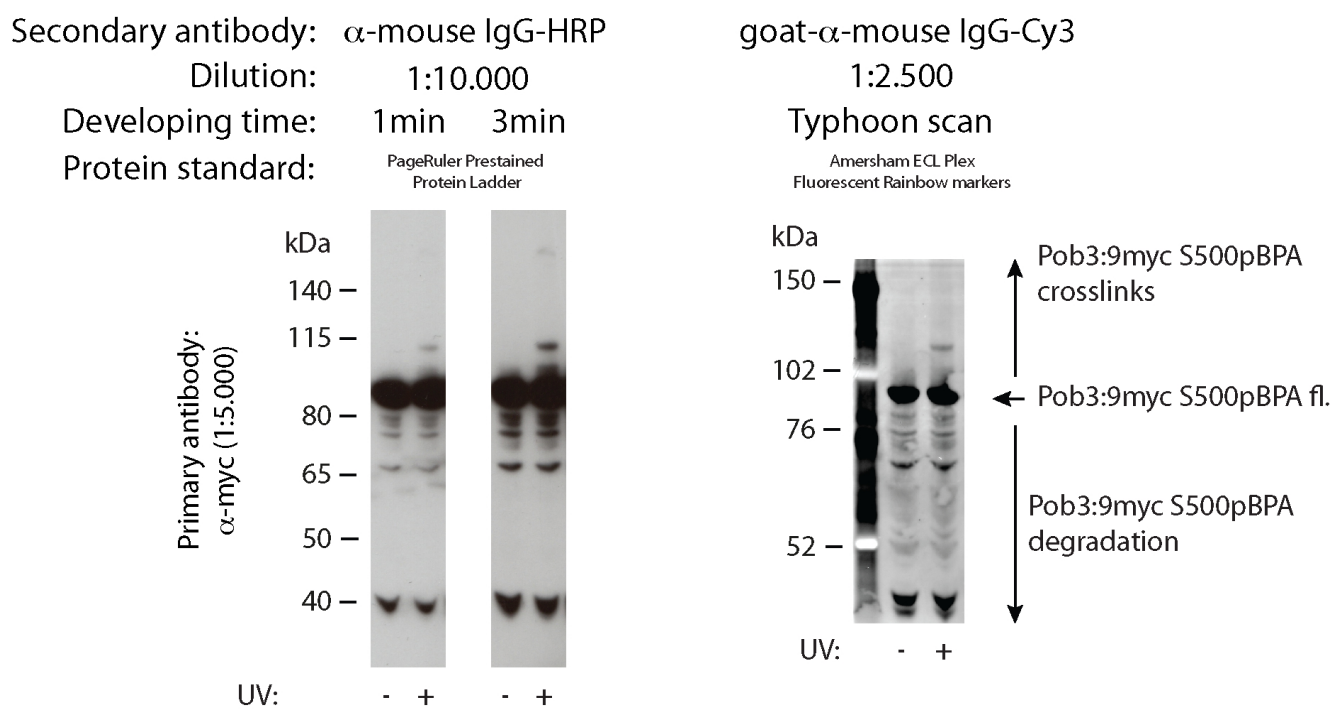

Figure 3.4: Comparison between standard chemiluminescent and fluorescent western blot detection - In vivo crosslinking experiments were performed with the Pob3:9myc S500TAG construct (7 minutes irradiation). Samples were prepared by boiling for 5 minutes at $95^{\circ} \mathrm{C}$ and further analyzed by SDS-PAGE (4- $12 \%$ NuPage $^{\circledR}$ gel, 500 mOD $_{600}$ per lane, Amersham Hybond LFP-membrane, $\alpha$-c-myc). After primary antibody incubation over night, the membrane was cut in half allowing an equal comparison. One half was incubated with $\alpha$-mouse IgG-HRP secondary antibodies (1:10.000 in $5 \%$ MLK/TBS) whereas the other half was decorated with $\alpha$-mouse IgG-Cy3 antibodies. For HRP-detection Amersham ${ }^{\text {TM }}$ $\mathrm{ECL}^{\mathrm{TM}}$ Prime Western Blotting Detection Reagent was used. Two different developing times are shown. The $\alpha$-mouse IgG-Cy3 decorated blot was detected with the Typhoon imaging system (Green laser: 532 nm; Cy3 filter 580 nm BP 30 nm; PMT 300; $25 \mu$ m pixel size; medium sensitivity).

The characteristic UV-dependent crosslink pattern is observed on western blots independent of their detection system. The chemiluminescent-developed western blot shows an intensive band for the Pob3:9myc full-length protein and the lowintense higher molecular weight crosslink at approximately $115 \mathrm{kDa}$. Although a longer developing time ( 3 minutes) could further increase the crosslink intensity, the full-length signal is saturated in this region of the blot. In contrast, the blot which was decorated with Cy3Dye-conjugated secondary antibodies shows better dynamic 
range and improved signal linearity indicated by the ratio of the crosslink to the fulllength Pob3:9myc protein. Another advantage is the reduced amount of consumables such as film and ECL ${ }^{\mathrm{TM}}$ substrate, since for the detection with the CyDye-conjugated secondary antibodies only the Typhoon imaging system is needed. Due to the better signal linearity, I decided to use the Amersham ${ }^{\mathrm{TM}} \mathrm{ECL}^{\mathrm{TM}}$ Plex system for further analysis, especially quantitative analysis.

\subsubsection{Comparison among sample preparation techniques: Yeast Protein Extraction Reagent, TCA precipitation and crude extract preparation}

Several sample preparation techniques were performed and tested for their applicability and quality. In vivo pBPA crosslinking experiments were performed with the Pob3:9myc Q310TAG construct. Samples were either prepared using either the Y-PER Yeast Protein Extraction Reagent and subsequent TCA precipitation or cells were directly subjected to TCA precipitation. These two were compared to the preparation of crude extracts by boiling the cells at $95^{\circ} \mathrm{C}$ for ten minutes (common). Additionally, the influence of a DNaseI treatment for sample quality was examined (figure 3.5).

All tested methods can be successfully used for the preparation of whole cell lysate samples for western blot. The characteristic UV-dependent crosslink pattern at the Q310 position is observed independently of the sample preparation method. This pattern consists of two prominent crosslinks at $140 \mathrm{kDa}$ and at $225 \mathrm{kDa}$. Differences in sample preparation concerning sample quality can be mainly observed for protein degradation. The Y-PER/TCA method results in a relatively high level of protein degradation in comparison to the boiling method with a moderate level. The TCA preparation without prior Y-PER lysis has the lowest level of protein degradation which is also indicated by the highest level of Pob3:9myc full-length protein recovery. The treatment with DNaseI had no effect of sample quality at the tested Q310 position. For further experiments, I performed preparation of whole cell extracts using the TCA method as described in the material and methods part (2.2.2.1).

\subsubsection{Crosslinking experiments in protease deficient yeast strains decrease protein degradation}

In order to analyze the degradation that appears during the sample preparation, I compared crosslinks at two pBPA incorporation sites (Pob3:9myc D260TAG and S500TAG) in different yeast strains. In addition to the wild-type BY4741 strain, the protease deficient strain DSY5 was tested. DSY5 has deletions of the two major vacuolar proteases (Pep4 and Prb1) which should minimize protein degradation 


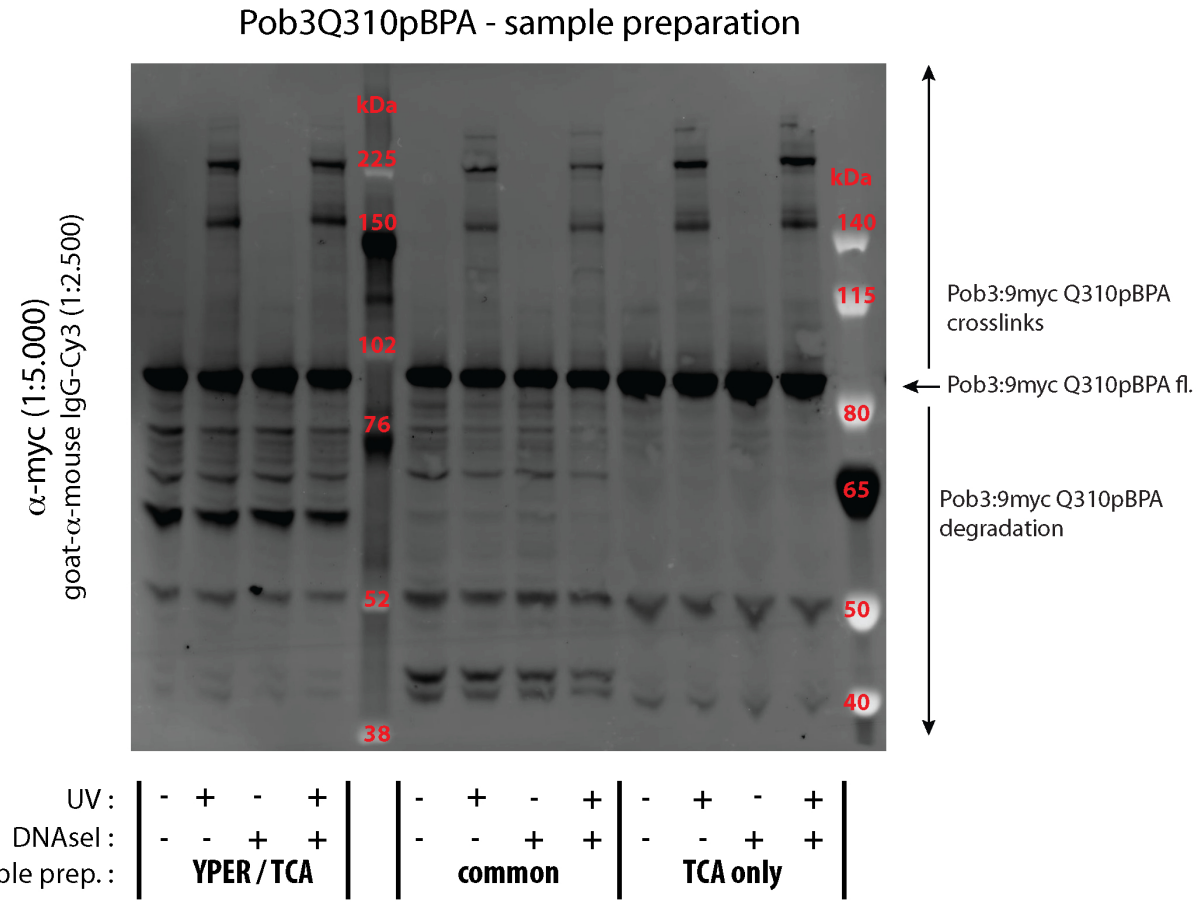

Figure 3.5: Comparison among different sample preparation techniques for western blot samples - In vivo crosslinking experiments were performed with the Pob3:9myc Q310TAG construct (7 minutes irradiation). Samples were either resuspended in Y-PER (for Y-PER/TCA samples) or in $\mathrm{H}_{2} \mathrm{O}$, both supplemented with $1 \mathrm{mM}$ PMSF, $1 \times$ PIC and with or without $180 \mathrm{U} / \mathrm{mL}$ DNaseI. Samples were incubated for 20 minutes at room temperature and subsequently TCA precipitated or boiled at $95^{\circ} \mathrm{C}$ for 10 minutes (common). The lysates were further analyzed by SDS-PAGE and western blot. (4-12 \% NuPage ${ }^{\circledR}$ gel, 500 mOD $_{600}$ per lane, Amersham Hybond LFP membrane, $\alpha$-c-myc primary and Cy3-conjugated secondary antibody, Typhoon scan (Cy3-settings, PMT 400; $50 \mu \mathrm{m}$ pixel size; high sensitivity).

during cell lysis. Crosslinking experiments were performed in parallel as indicated in the figure legend (figure 3.6).

After induction with galactose, the DSY5 strain grew significantly slower and reached only half of the $\mathrm{OD}_{600}$ compared to the BY4741-a cells. As expected, for all tested positions and cell types the addition of pBPA to the cultures substantially increased the full-length Pob3:9myc protein. An additional UV-treatment of these cells shows a characteristic crosslink pattern at both positions (lane 3 and lane 10). Although the samples were normalized to the same $\mathrm{OD}_{600}$, DSY5 cells expressing Pob3:9myc pBPA mutants show less full-length protein compared to the samples from BY4741 cells. Therefore, the crosslink patterns are less intense but in case of position S500 still reproducible (lane 3 and 6). Interestingly, the levels of full-length Pob3:9myc is comparable in both yeast strains in the absence of pBPA. However, samples from DSY5 cells show tremendously reduced protein degradation compared to samples from BY4741 cells. This can be especially observed comparing the nonBPA, non-UV-treated samples of the two different strains (lane 1 and 5). Although 
DSY5 samples show less degradation effects, I decided to use the BY4741 strain since it grows better.

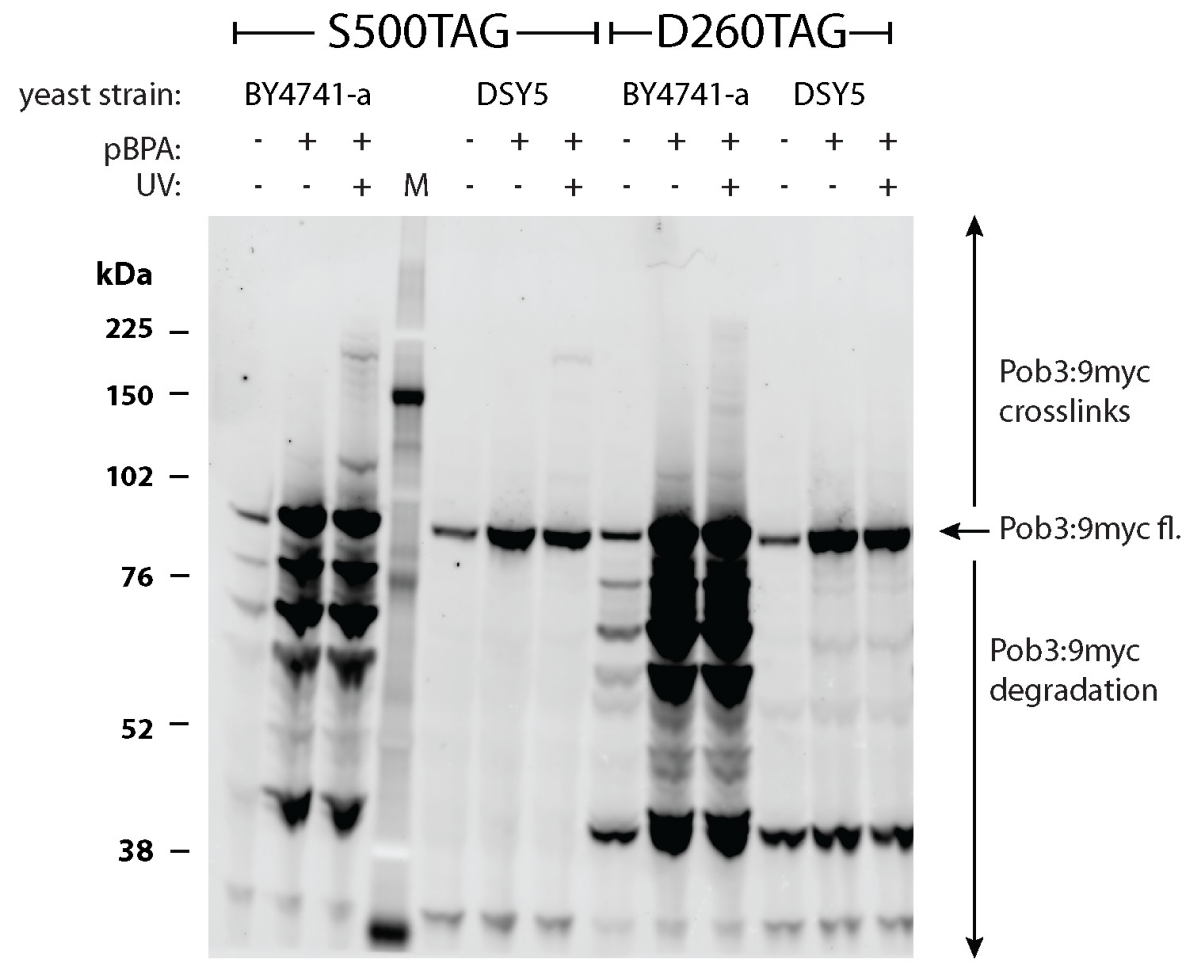

Figure 3.6: Crosslinking experiments in a protease deficient yeast strain - In vivo crosslinking experiments were performed with the Pob3:9myc D260TAG and S500TAG construct. In addition to the BY4741 strain, experiments were performed in the Pep4/Prb1-protease-deficient yeast strain, DSY5 (7 minutes irradiation). Samples were boiled for 10 minutes at $95^{\circ} \mathrm{C}$ and analyzed by SDS-PAGE and western blot $\left(4-12 \%\right.$ NuPage $^{\circledR}$ gel, $500 \mathrm{mOD}_{600}$ per lane, Amersham Hybond LFP-membrane, $\alpha$-c-myc primary and Cy3-conjugated secondary antibody, Typhoon scan (Cy3-settings; PMT 400; $50 \mu \mathrm{m}$ pixel size; medium sensitivity).

\subsubsection{Crosslinking screen of the yFACT complex using the Spt16 and Pob3 amber suppression libraries}

After optimization, experiments were extended to a comprehensive crosslinking screen using a Spt16 and Pob3 amber library. For generation of Spt16 and Pob3 amber library mutants, I performed Quikchange mutagenesis PCR. The parent plasmids for the Quikchange mutagenesis PCR contain either the Spt16-ORF as a fusion construct to the 9 myc tag under the control of the endogenous promoter $(-500 \mathrm{bp}$ from the start codon, p426 E Spt16:9myc) or the Pob3-ORF as a fusion to the 9myc tag under the control of the GALS promoter (p426 GAL Pob3:9myc, see section 2.2.1.12). The 
library cloning and subsequent analysis was done in a high-throughput fashion. Primers were purchased in 96-well blocks. Quikchange PCR reactions, as well as E. coli transformations and cultures for plasmid preparations, were performed in similar scales. Using this approach, I replaced approximately every tenth sense codon with TAG at rapid pace in both Spt16 and Pob3. Time frame from the library primer design to the first western blot readouts lasts about 2 month. The final library consist of 214 individual FACT complex mutants (128 for Spt16; 86 for Pob3). The full list of constructs and their corresponding mutagenesis primers is shown in the appendix of this thesis (see supplementary table 5.2.).

\subsubsection{Scanning of the FACT complex with the genetically encoded UV-inducible crosslinker pBPA in Saccharomyces cerevisiae}

The site-specificity of the in vivo crosslinking technique using the genetically UVinducible crosslinker pBPA allows the investigation of the interaction pattern at the single amino acid resolution. Repositioning of the crosslinker, thus screening through the protein, would reveal the global interaction landscape at a high resolution. Hence, I performed crosslinking experiments at 119 different sites of Spt16 and 67 different sites of Pob3 respectively. Positions were selected supported by structural data if available. This screening of the entire Spt16 and Pob3 library was done in a highthroughput way utilizing yeast culturing and crosslinking in 48-deepwell blocks. All experiments were performed in the BY4741 background which contained the pESC pBPA-RS (LEU) plasmid for incorporation of pBPA in response to the amber suppression codon. The comprehensive crosslinking study is shown in figure 3.7 .

For most of the sites of pBPA incorporation, I observe comparable amounts of full-length Spt16:9myc or Pob3:9myc protein. Furthermore, a reproducible pattern of crosslinks, indicated by a higher molecular weight than the free protein, is detected for the majority of tested crosslink positions. The crosslink formation is UV-depended which I confirmed for several positions (figure 3.8). Adjacent crosslinking sites show frequently similar banding patterns. This suggests potential interaction surfaces with similar partners (for example: Spt16 R875-D908; Y971-D996; Pob3 S500-E537). Furthermore, many distinct crosslinked products which are very specific for a certain region of the protein can be observed in both screens: Spt16-F250, F596, T713, R875, E976, W1017 and Pob3-I180, R254, K271 and S500. Some crosslinked products are highly abundant and are present almost throughout the entire scan. They may represent interactions to proteins involved in more general biological roles such as protein biosynthesis, folding or degradation. 
A. Spt16-pBPA scan

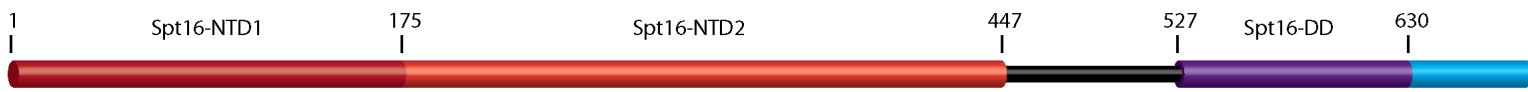

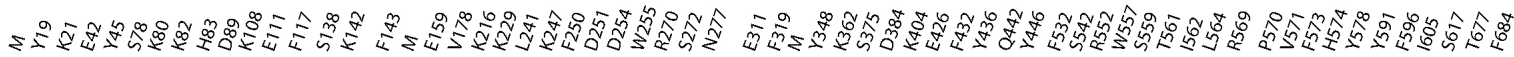
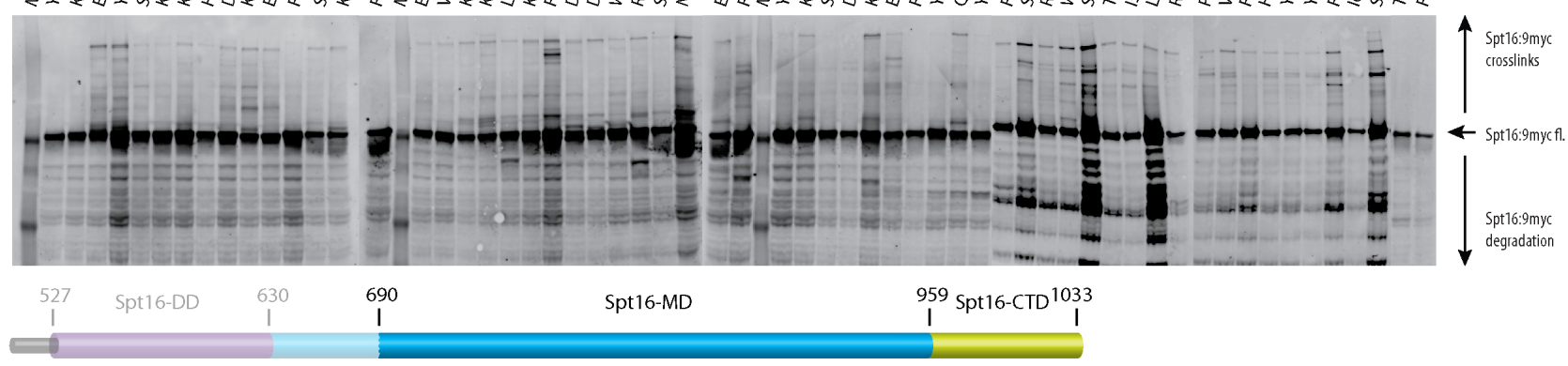

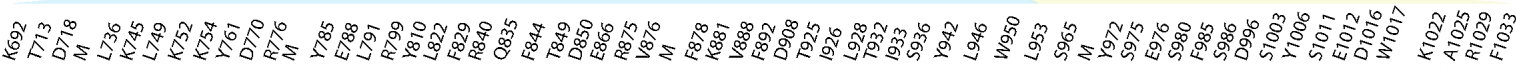

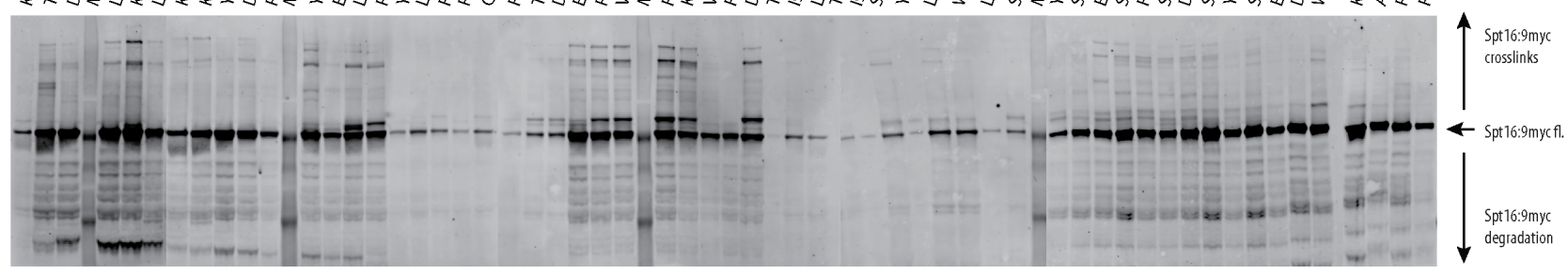

B. Pob3-pBPA-scan

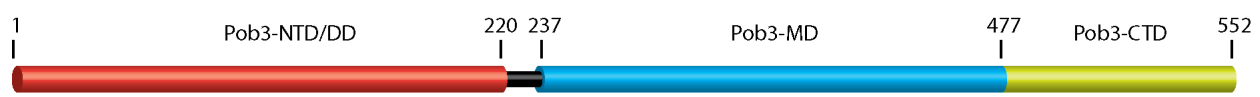

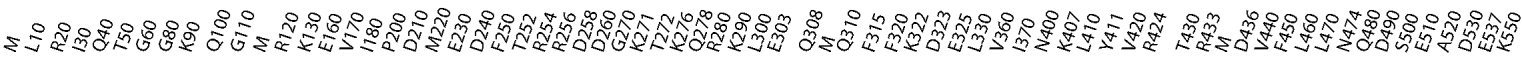

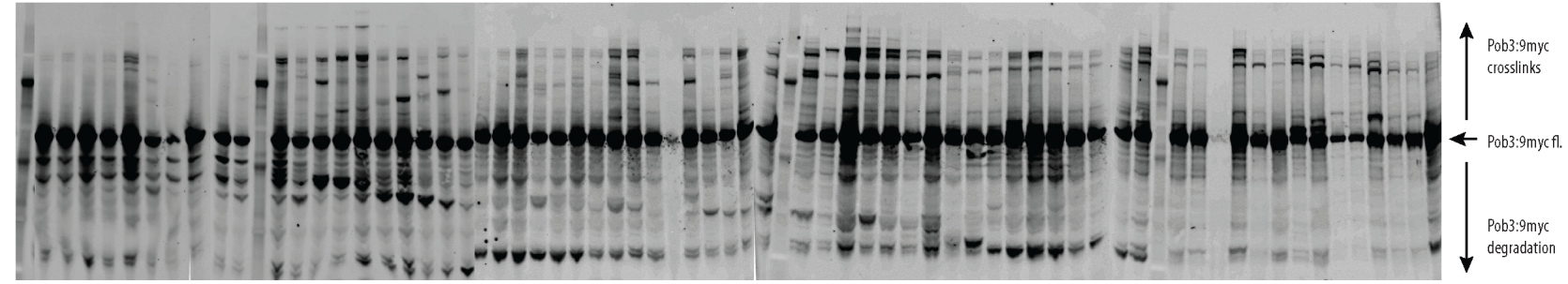

Figure 3.7: Scanning of the FACT complex with the genetically encoded UV-inducible crosslinker pBPA in Saccharomyces cerevisiae - A. pBPA scan of Spt16: Samples of cells (BY4741) expressing Spt16:9myc under the control of the endogenous promoter with pBPA genetically encoded at 119 different sites were subjected to UV irradiation (20 minutes) and analyzed by SDS-PAGE and western blot (3-8 \% Tris-Acetate gels; $600 \mathrm{mOD}_{600}$ per lane (TCAprecipitated), Amersham Hybond LFP-membrane, $\alpha$-c-myc primary and Cy3-conjugated secondary antibody, Typhoon scan (Cy3-settings; PMT 400; $50 \mu \mathrm{m}$ pixel size; medium sensitivity). Substituted amino acids are assigned to a schematic representation of the Spt16 protein. Each lane represents the trapped interactions at the specific substituted site. Observed repetitive crosslinks might indicate an interaction surface of a distinct protein. B. pBPA scan of Pob3: In contrast to Spt16-scan (A.), Pob3:9myc was expressed under the control of the inducible GAL1 promoter and pBPA was incorporated at 67 different sites (15 minutes irradiation). Samples were analyzed by SDS-PAGE and western blot (4-12 \% Bis-Tris gels, 600 mOD $_{600}$ per lane (cells were boiled for 10 minutes at $95^{\circ} \mathrm{C}$ ), Typhoon scan (Cy3-settings); amino acids L10-D240: PMT 400; $50 \mu \mathrm{m}$ pixel size; medium sensitivity; amino acids F250-K550: PMT 300; $100 \mu \mathrm{m}$ pixel size; high sensitivity). 
A. Pob3:9myc

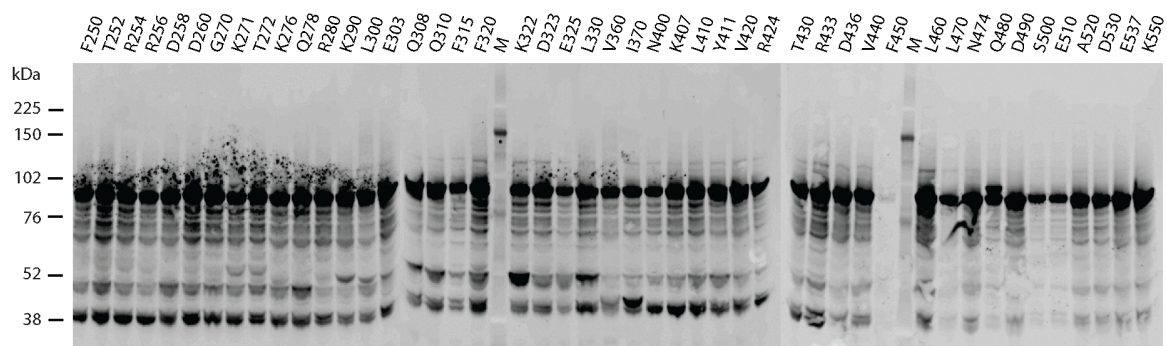

B. Spt16:9myc

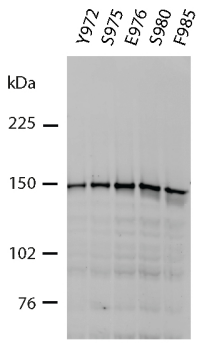

Figure 3.8: Controls for the FACT complex pBPA scan in Saccharomyces cerevisiae - Selected cells from the pBPA scan (shown in figure 3.7) of Pob3 (A) and Spt16 (B) were not irradiated with UV-light. Samples were processed and analyzed precisely as described above.

\subsubsection{Characterization of the amber suppression libraries using temperature sensitive yeast strains}

The functionality test of the produced pBPA-containing mutant variants of Spt16 or Pob3 was performed using a rescue assay in temperature sensitive spt16/pob3 yeast strains. Experiments were done by plating serial dilutions of each pBPA mutant on media with or without $\mathrm{pBPA}$ and incubating them at restrictive $\left(37^{\circ} \mathrm{C}\right.$ for $s p t 16$ - $t s$ and $33^{\circ} \mathrm{C}$ for pob3-L78R) or permissive temperature $\left(25^{\circ} \mathrm{C}\right)$. The general figure 3.9 contains a selection of mutants to clarify the results using a rating system. The complete library screens are shown in figure 3.10 for Spt16 and in figure 3.11 for Pob3. The complete classifications of the entire amber library are depicted in the appendix of this thesis in table 5.3 and table 5.4 .

The spt16-ts strain was verified by sequencing and contained in addition to the G132D mutation a E1013G mutation. It grew normal at the permissive temperature whereas it shows pBPA-independent inviability at $37^{\circ} \mathrm{C}$. Additional plasmid borne expression from the pRS426 Spt16:9myc plasmid fully recovered the growth sensitive phenotype. Spt16 amber mutants showed a range of responses to pBPA-dependent rescue ability. Some positions, such as K82TAG or D908TAG, were classified with the best rescue ability (i.e. four pluses on the scale). Followed by positions such as D251 or D718, which showed a general lower rescue ability. In fact, the majority of the Spt16 mutants containing the UV-inducible crosslinker at the indicated positions can rescue the spt16-ts mutant phenotype and can be claimed functional (figure 3.10 and table 5.3). Interestingly, rescue ability was observed for the majority of the sites also in the absence of the unnatural amino acid.

Surprisingly, positions in the Spt16 middle domain, which showed reduced amount of full length protein in the Spt16-BPA screen western blot analysis (figure 3.7, positions Y810-D850 and T925-S965; except 1926, Q938 and D940), completely failed to rescue the temperature sensitive spt16-ts phenotype. Moreover, this loss of rescue 
A. Rescue assay in spt16-ts

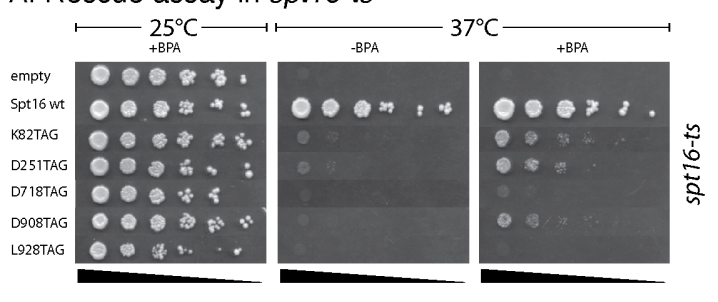

\begin{tabular}{|c|c|c|c|c|}
\hline position & $25^{\circ} \mathrm{C}+\mathrm{pBPA}$ & $37^{\circ} \mathrm{C}-\mathrm{pBPA}$ & $37^{\circ} \mathrm{C}+\mathrm{pBPA}$ & rescue \\
\hline empty & ++++++ & 0 & 0 & 0 \\
\hline wt & ++++++ & ++++++ & ++++++ & 0 \\
\hline K82 & ++++++ & ++ & +++++++ & ++++ \\
\hline D251 & ++++++ & ++ & ++++ & ++ \\
\hline D718 & +++++ & 0 & + & + \\
\hline D908 & ++++++ & 0 & ++++ & ++++ \\
\hline L928 & +++++ & 0 & 0 & 0 \\
\hline
\end{tabular}

B. Rescue assay in pob3-L78R

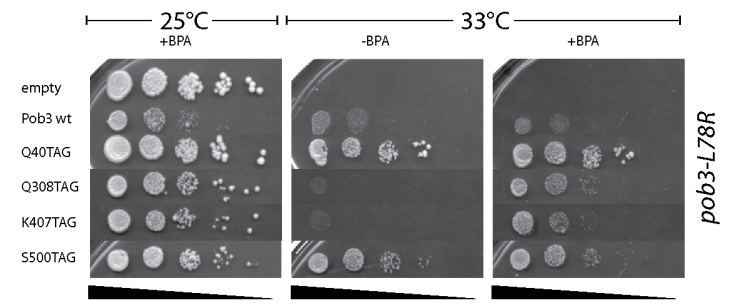

\begin{tabular}{|l||c|c|c|c|}
\hline position & $25^{\circ} \mathrm{C}+\mathrm{pBPA}$ & $33^{\circ} \mathrm{C}-\mathrm{pBPA}$ & $33^{\circ} \mathrm{C}+\mathrm{pBPA}$ & rescue \\
\hline empty & +++++ & 0 & 0 & 0 \\
wt & +++ & ++ & ++ & 0 \\
Q40 & +++++ & ++++ & ++++ & 0 \\
Q308 & +++++ & 0 & +++ & +++ \\
K407 & +++++ & 0 & +++ & +++ \\
S500 & +++++ & ++++ & ++++ & 0 \\
\hline
\end{tabular}

Figure 3.9: pBPA-dependent rescue assay testing selected Spt16/Pob3-pBPA mutants for functionality in temperature sensitive yeast strains - A: Spt16-ts cell containing the plasmid for incorporation of pBPA (pESC pBPA-RS LEU) were cotransformed with plasmids from the Spt16:9myc amber library. For controls, an empty plasmid (pRS426) and a plasmid with the wild-type Spt16:9myc ORF were used. Cells were plated as 1:10 serial dilutions starting with an $\mathrm{OD}_{600}$ of 2.0 on selective media plates with or without $2 \mathrm{mM}$ pBPA. Plates contained $2 \%$ glucose as carbon source and were incubated either at permissive $\left(25^{\circ} \mathrm{C}\right)$ or restrictive $\left(37^{\circ} \mathrm{C}\right)$ temperature and analyzed 5 days after spreading. B: A similar functionality test of the Pob3 amber suppression library was performed using the pob3-L78R strain. The dilutions were spread on plates containing $2 \%$ galactose and $1 \%$ raffinose and incubated either at permissive $\left(25^{\circ} \mathrm{C}\right)$ or restrictive $\left(33^{\circ} \mathrm{C}\right)$ temperature. Plates were analyzed 10 days after incubation.

is fully restored downstream at the CTD of Spt16 where the full rescue ability is observed independently of pBPA addition (Spt16 S980-F1033). Hence, the truncated fragments by termination at the amber codon complements for the effects of the ts-mutation.

The functionality test of the Pob3 amber library showed a similar outcome. The pob3-L78R strain was verified by sequencing and had several additional mutations despite the annotated L78R mutation: N187D, N331D and E503D mutation. The pob3-L78R temperature sensitive phenotype results from a loss of Spt16-Pob3 heterodimers due to protein instability 182 . Plasmid-borne Pob3:9myc expressed from p426 GAL Pob3:9myc plasmid was able to rescue the temperature sensitive pob3- $L 78 R$ phenotype at restrictive temperature (figure 3.9). However, expression of Pob3:9myc already impaired growth at $25^{\circ} \mathrm{C}$. This confirms the observed growth effects where I saw increased doubling-time in liquid culture (see section 3.1.2). Several amber mutants showing this behavior too (Pob3-M D240-Q278 and Pob3-CTD L470-S491; figure 3.11 and table 5.4. Nevertheless, roughly 66 out of 86 positions show normal growth at permissive temperature and rescued the pob3- $L 78 R$ phenotype. Half of those revealed additional pBPA dependency. Besides Spt16, a full pBPA-independent 

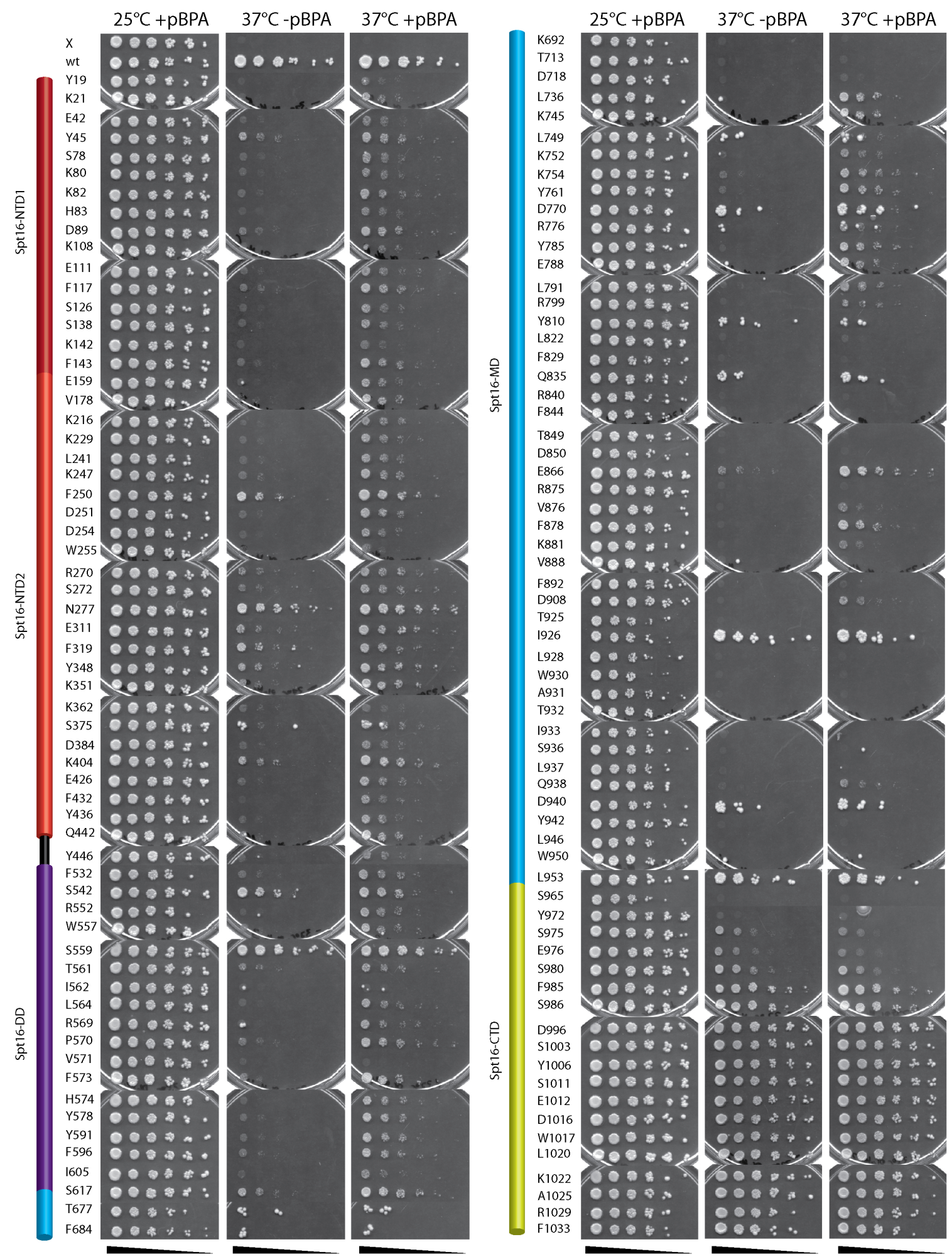

Figure 3.10: pBPA-dependent rescue assay testing Spt16-pBPA mutants for functionality in spt16ts strain - For experimental details see the classification figure 3.9 A. 


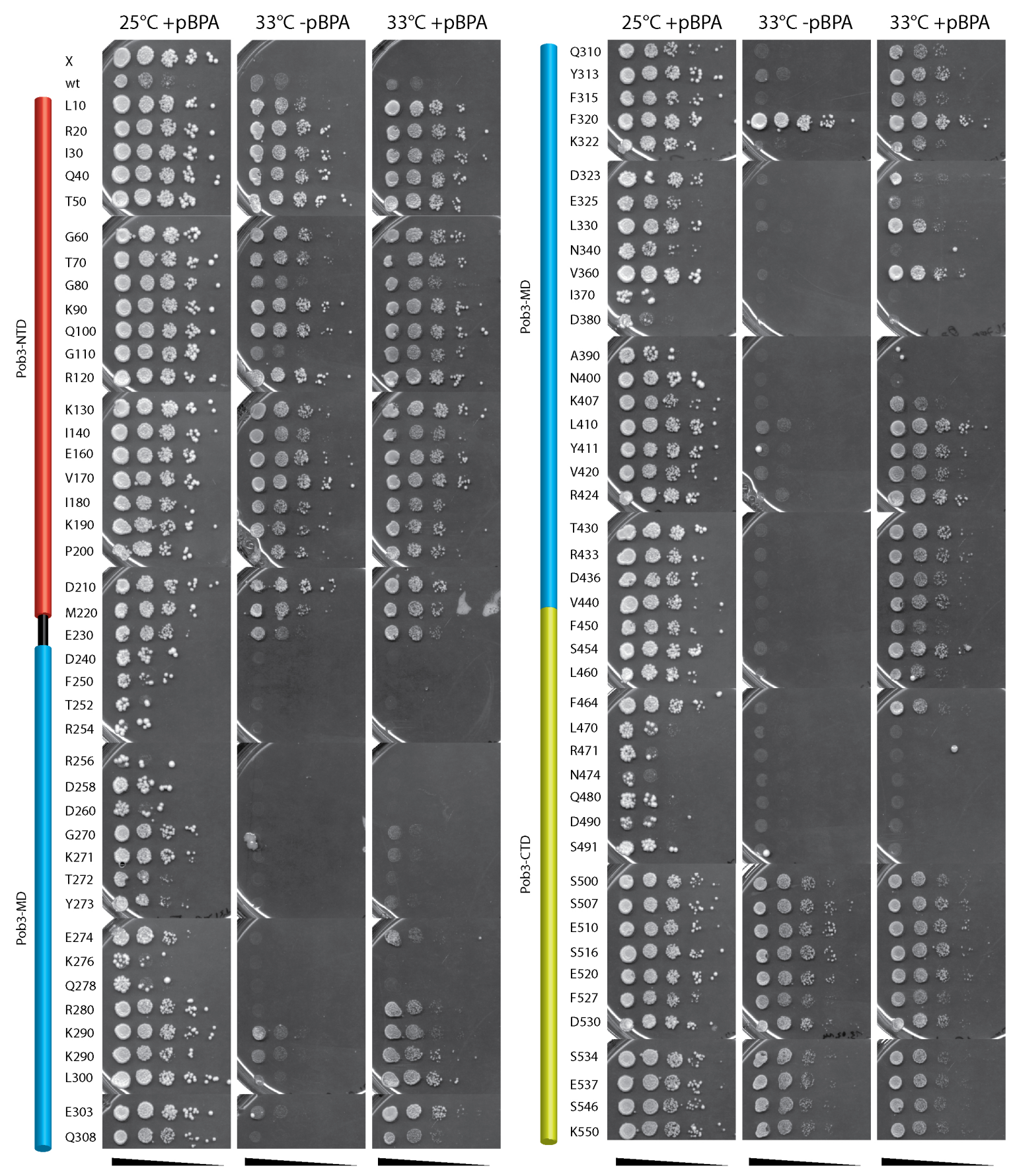

Figure 3.11: pBPA-dependent rescue assay testing Pob3-pBPA mutants for functionality in pob3L78R strain - For experimental details see the classification figure $3.9 \mathrm{~B}$. 
rescue ability at the CTD of Pob3 can be observed. From these experiments, I conclude that the vast majority of the produced FACT complex amber pBPA mutants can rescue the temperature sensitive phenotype of the respective protein and hence are functional in vivo.

\subsection{The in vivo interactions between FACT and histones}

Next I set out to analyze the FACT complex for interactions with histones. This is crucially important since the crystal structure of the entire complex is not available and current interaction studies of single domains only reflect the simplified in vitro environment. I started the search with crosslinked products which produce a molecular weight shift of approximately 10-20 kDa to the full-length protein. Additionally, I developed an easy identification strategy of the unknown crosslinked products by molecular weight shift assays.

\subsubsection{Characterization of the acidic C-terminal tail serine $\mathbf{5 0 0}$ of Pob3}

Crosslinking at S500 of Pob3 results in a molecular weight shift of 10-20 kDa for the full-length Pob3 protein. Moreover, the S500 is located in the C-terminal domain (CTD) of Pob3 which is thought to interact with histones by outcompeting the negatively charged DNA. Our in vivo approach represents an excellent strategy to test histone interactions in this domains in yeast. Hence, I analyzed the CTD of Pob3 in more detail.

\subsubsection{Identification of the S500 crosslinking product using molecular weight shift assays}

The identification of an unknown crosslink can be either done by immunoprecipitation and subsequent mass-spectrometry or by the molecular weight shift approach (figure $3.12 \mathrm{~A}$ ). The shift-assay is a cost- and time-effective technique for the identification of crosslinked products. The candidate approach works by duplication of the crosslink experiment in a yeast strain where the putative interaction partner is genomically tagged (figure 3.12 A. red protein tag). Hence, a positive candidate would result in an additional molecular weight shift in a western blot.

Prior to the experiments, yeast strains with genomically tagged histones were tested for functionality (figure $3.12 \mathrm{~B}$ ). Cells harboring genomically tagged copies 
A. Molecular weight shift approach

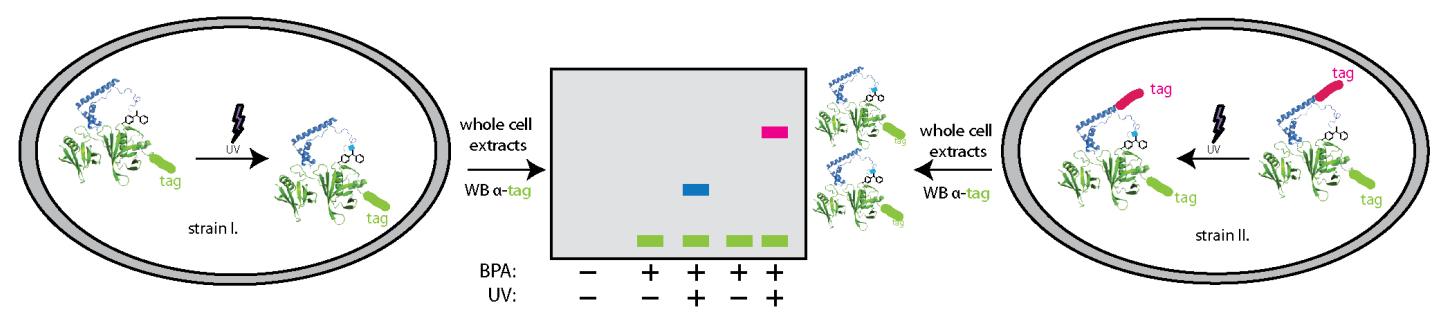

B. Yeast strains for shift approach

C. Molecular weight shift for Pob3 S500pBPA
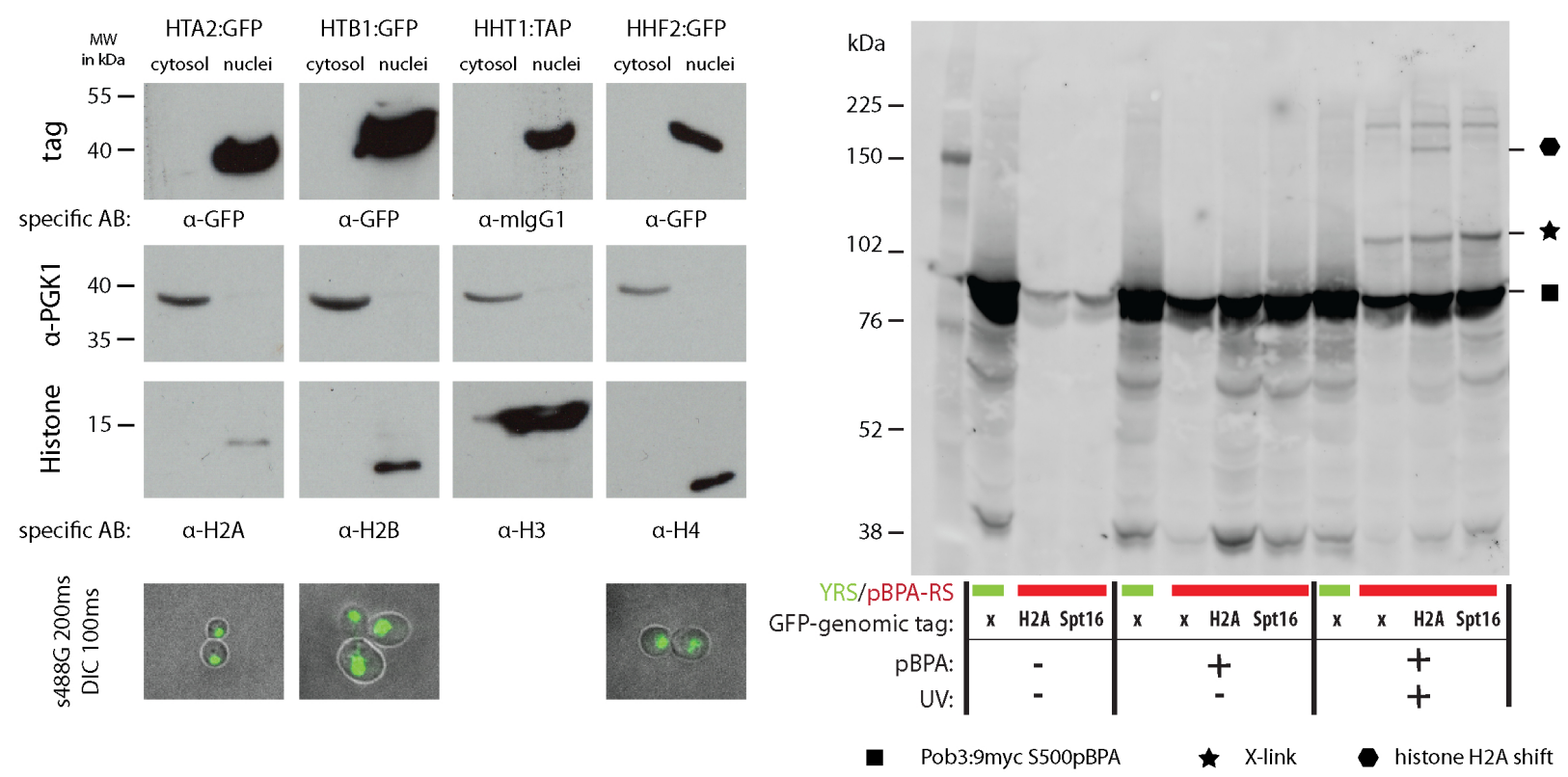

Figure 3.12: Molecular weight shift approach and identification of an Pob3-H2A crosslink at serine 500 - A: The molecular weight shift approach is performed by two individual crosslink experiments in different yeast strains. Strain II. contains a genomically tagged variant of the assumed candidate crosslink partner which would result in an additional molecular weight shift in a crosslink experiment using this strain. B: Yeast strains for shifting experiments with different genomically tagged histones were tested for their integrity. Strain backgrounds are shown in table 2.1. Yeast cells $\left(75 \mathrm{OD}_{600}\right)$ were subjected to nuclear preparation, TCA precipitated and analyzed by $15 \%$ SDS-PAGE. Western blots were performed against the specific tag antibody ( $\alpha$-GFP 1:000 for GFP or $\alpha$-mIgG1-HRP (1:1.000 in $5 \%$ MLK/TBS) for the TAP-tag), the specific histone and against phosphoglycerate kinase. Presence of PGK in the cytosol confirms successful fractionation. All protein tags of the tested histone fusion tag yeast strains were verified. Additional fluorescence microscopy of the histone-GFP fusion strains confirmed nuclear GFP localization (s488G: $200 \mathrm{~ms}$; DIC: $100 \mathrm{~ms}$; laser power: $100 \%$; gain: 1; intensification: 200). C: Crosslink experiments were performed in BY4741 (x) wild-type yeast cells and cells containing a genomically GFP-tagged version of H2A or Spt16. Cells contained the plasmid for amber suppression of Pob3:9myc S500TAG and either the non-evolved tRNA-synthetase from E.coli for incorporation of tyrosine (YRS) or the evolved tRNA-synthetase (pBPA-RS) for incorporation of pBPA (10 minutes irradiation). Total proteins were TCA precipitated, analyzed by SDS-PAGE and western blot (4-12 \% Bis-Tris Gels; $\alpha$-c-myc primary and Cy3-conjugated secondary antibody, Typhoon scan (Cy3-settings; PTM 350; $50 \mu \mathrm{m}$ pixel size; high sensitivity). 
were subjected to subcellular fractionation and lysates were analyzed by SDS-PAGE and western blot. For H2A:GFP, H2B:GFP and H4:GFP the tag specific $\alpha$-GFP antibody was used. For H3, a strain with a fusion of HHT1 with the TAP-tag was used and detection was done with $\alpha$-mIgG1 antibody. Furthermore, live cell fluorescence microscopy was done for the genomic GFP fusion proteins. For all four tested yeast strains, the tag-specific and histone-specific signal moves into the nuclei fraction, whereas the phosphoglycerate kinase is depleted in the nucleus. This ensures the presence and the proper localization of the genomically encoded histone fusion proteins. Additionally, for the GFP-strains a strong nuclear localization can be seen under the microscope. Together, these strains can be used for molecular weight shift assay.

Crosslink experiments of Pob3:9myc with the amber codon at position S500 were performed in the BY4741 wild-type yeast strain (x), a strain with genomically tagged H2A:GFP and a strain with genomically tagged Spt16:GFP as a control. In addition

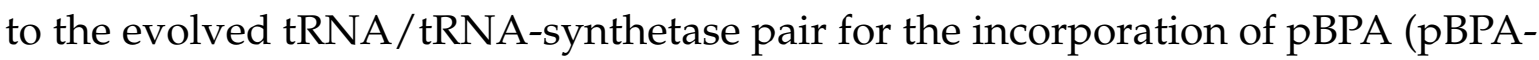
$\mathrm{RS})^{255}$, I performed an experiment using the non-evolved tRNA-synthetase of E.coli for tyrosine (YRS). This resulted in pBPA-independent full-length Pob3 expression by incorporation of tyrosine in the absence of pBPA in the medium. In contrast, the samples containing the tRNA/tRNA-synthetase pair for the incorporation of pBPA (BPA-RS) showed expression of full-length Pob3 in the presence of pBPA. The formation of crosslinked products was only observed for cells expressing the pBPA-RS. No crosslink formation was observed for cells containing the non-evolved tRNA-synthetase of E.coli for tyrosine (YRS). Hence, only the incorporation of pBPA at S500 allows crosslink formation. Most strikingly, a protein band of higher molecular weight of roughly $30 \mathrm{kDa}$ is only observed on the western blot in samples where H2A was genomically tagged with GFP. Thus, this additional shift clearly indicates that the pBPA incorporated at S500 in Pob3 crosslinked to the histone H2A in vivo. However, I could not identify an interaction with the FACT complex partner Spt16 at this position.

With this experiment, I was able to detect and locate the first in vivo interaction between histone $\mathrm{H} 2 \mathrm{~A}$ and Pob3 at serine 500. This motivated me to analyze this region in more detail.

\subsubsection{Localization of the formed crosslinking products at S500}

In order to determine the subcellular localization of the Pob3S500-H2A interaction, I performed crosslinking experiments and subjected the samples to fractionation. Previously, western blot analysis of crosslinking experiments was performed with 
whole cell lysates preventing any conclusion concerning the subcellular distribution. Now, cells were digested with lyticase and mechanically disrupted with a dounce homogenizer. Fractions were collected by ficoll density gradient centrifugation. The samples were analyzed by SDS-PAGE and western Blot (figure 3.13).

The whole cell extract (WCE) sample which was a positive control showed the UV-depended Pob3-H2A interaction (figure 3.13 A. for replicate 1). The samples of the subcellular fractionation showed that the Pob3-S500BPA-H2A crosslink is predominantly found in the nuclear fraction whereas only little amounts were detected in the cytosolic fraction. The quality of the fractionation was tested by western blot against epitopes of cytosolic or nuclear marker proteins; phosphoglycerate kinase 1 (PGK1) and histone 3. Since PGK1 was depleted and H3 was enriched in the nucleus, I can rely on this experiment as a high quality fractionation. A densitometrically quantification was done by titrating all three biological replicates (figure $3.13 \mathrm{C}$. and D.). The density of the crosslink in the nucleus is determined as a tenth of the full-length Pob3-S500pBPA protein, compared to a fortieth in the cytosol (figure 3.13 B.). The crosslink shows a significant $\sim 4$ fold enrichment in the nuclei in respect to the cytosol normalized to the amount of full-length Pob3:9mycS500pBPA. This difference is statistically highly significant. This crucial control of the novel H2A-Pob3 interaction ensured the integrity of the experiment indicating a functional interaction of the FACT complex in the nucleus with chromatin.

\subsubsection{Comparison among two genetically encoded UV-inducible crosslinker amino acids: pBPA and pAzF}

Since the integrity of the crosslinking approach for the Pob3S500-H2A interaction was proven, I wanted to exclude the effect of the pBPA-crosslinking amino acid by itself. The rather bulky and hydrophobic side chain may affect protein structure at the site of incorporation and therefore might induce a false positive interaction, respectively crosslink. Therefore, I tested an alternative light-responsive crosslinker amino acid, 4-Azido-L-phenylalanine (pAzF). I incorporated pAzF at serine 500 of Pob3 and compared its crosslinking ability to the previously used pBPA (figure 3.14 A.). I increased the crosslinking time to 30 minutes since previous experiments indicated reduced crosslinking yields using pAzF. Additionally, I performed molecular weight shift experiments using a genomically H2A:3myc-tagged and a H3:TAP-tagged yeast strain.

Full-length Pob3:9myc protein was detected for all samples with the pBPA- and the pAzF-system. Levels of protein expression were comparable in all strains (BY4741, H2A:3myc and H3:TAP). The Pob3S500-H2A crosslink was detected in 
A. Subcellular localization of Pob3S500pBPA-H2A crosslink

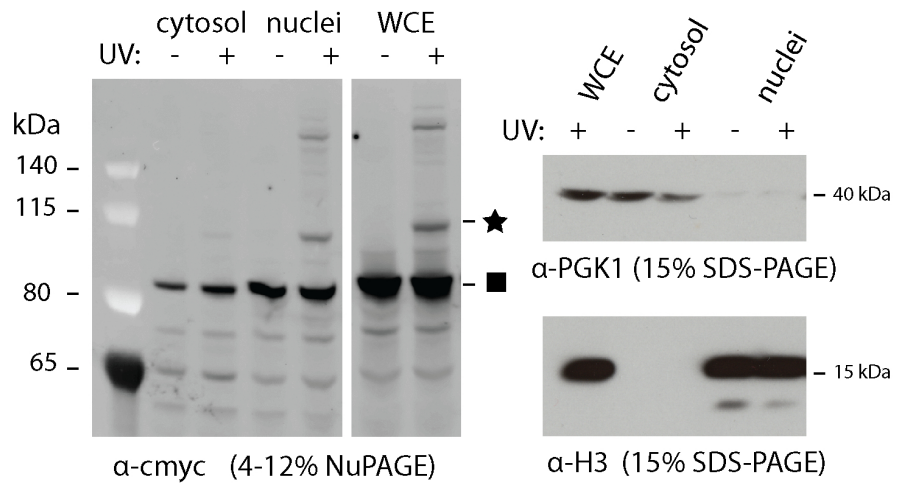

B. Quantification $(n=3)$

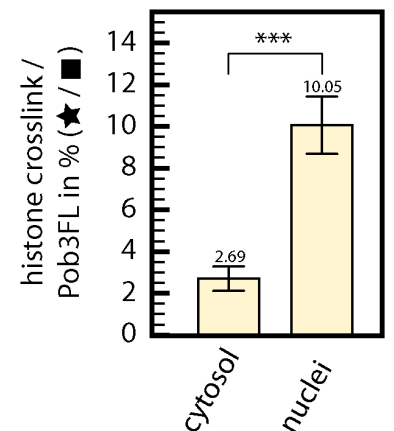

C. Titrations of subcellular localization of Pob3S500pBPA-H2A crosslink

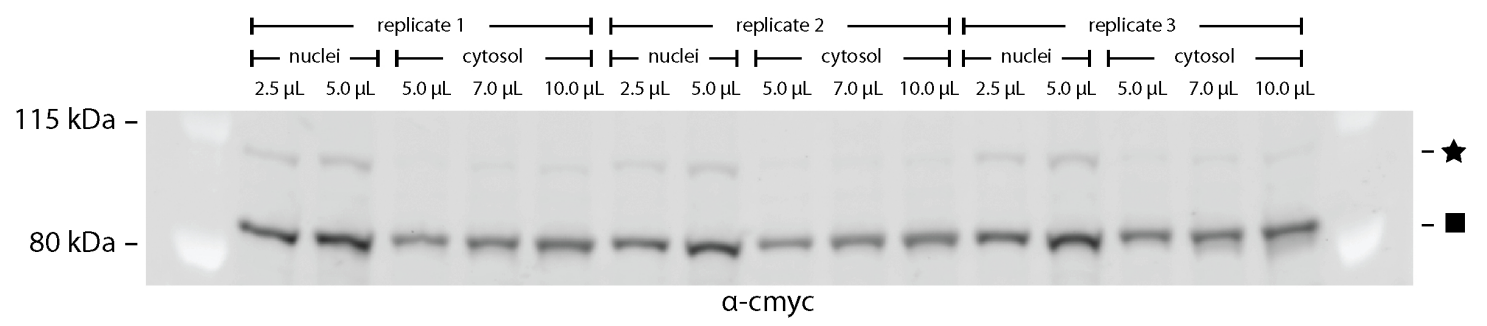

D. Quality control of subcellular fractionation

E. Pob3 expression levels
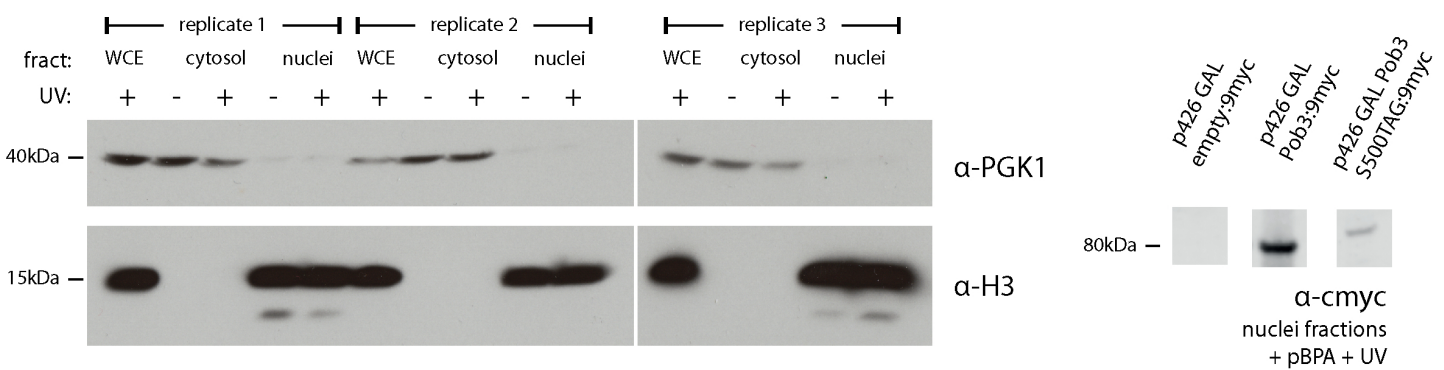

Pob3:9myc S500pBPA

Pob3-H2A crosslink

Figure 3.13: Subcellular localization and quantification of the Pob3-S500pBPA-H2A crosslink - A: Crosslink experiments of Pob3:9myc (three biological replicates) were performed at position serine 500 (20 minutes irradiation) and subjected to fractionation. Spheroplasts were prepared by lyticase digestion and fractions were obtained by ficoll density gradient centrifugation and TCA precipitated. Crosslinks were analyzed by western blot (4-12\% Bis-Tris Gels; Amersham Hybond-LFP membrane, $2.5 \mathrm{OD}_{600}$ per lane, $\alpha$-c-myc primary and Cy3-conjugated secondary antibody, Typhoon scan (Cy3-settings; PTM 400; $50 \mu \mathrm{m}$ pixel size; high sensitivity). WCE (whole cell extracts) samples were prepared by standard TCA precipitation $\left(800 \mathrm{mOD}_{600}\right.$ per lane). The quality of the fractionation was tested by western blot against epitopes of cytosolic or nuclear marker proteins; phosphoglycerate kinase 1 (PGK1) and histone 3 (15\% SDS-PAGE; PVDF membrane; 5 OD $_{600}$ per lane, $\alpha$ mouse/rabbit IgG-HRP secondary antibody, Amersham ${ }^{\mathrm{TM}} \mathrm{ECL}^{\mathrm{TM}}$ Prime Western Blotting Detection Reagent ( 3 seconds exposure)). As an example, one of the three replicates is shown. B: Quantitative analysis of the subcellular localization of the H2A:Pob3S500pBPA crosslink. Three independent experiments (see part C. and D.) were titrated and analysed using Fiji. Independent crosslink to full-length protein ratios were calculated for each titration point. Average and standard deviation was calculated for the cytosolic and nuclei titrations. The $\sim 4$ fold enrichment of the Pob3-histone crosslink normalized to the amount of full-length Pob3:9mycS500pBPA was statistically examined using the Student's $\mathrm{t}$-test (two-tailed distribution; two-sample equal variance; $\mathrm{p}=2.23^{*} 10^{-9}$; levels: ${ }^{*} \mathrm{p}<0.05$, ** $\left.\mathrm{p}<0.01,{ }^{* * *} \mathrm{p}<0.001\right)$. E: Crosslinking experiments in yeast harboring different expression plasmids (empty vector control, full-length Pob3:9myc and Pob3:9mycS500TAG). Cultures contained pBPA and all samples were irradiated. Western blot shows nuclear fractions. 
A.

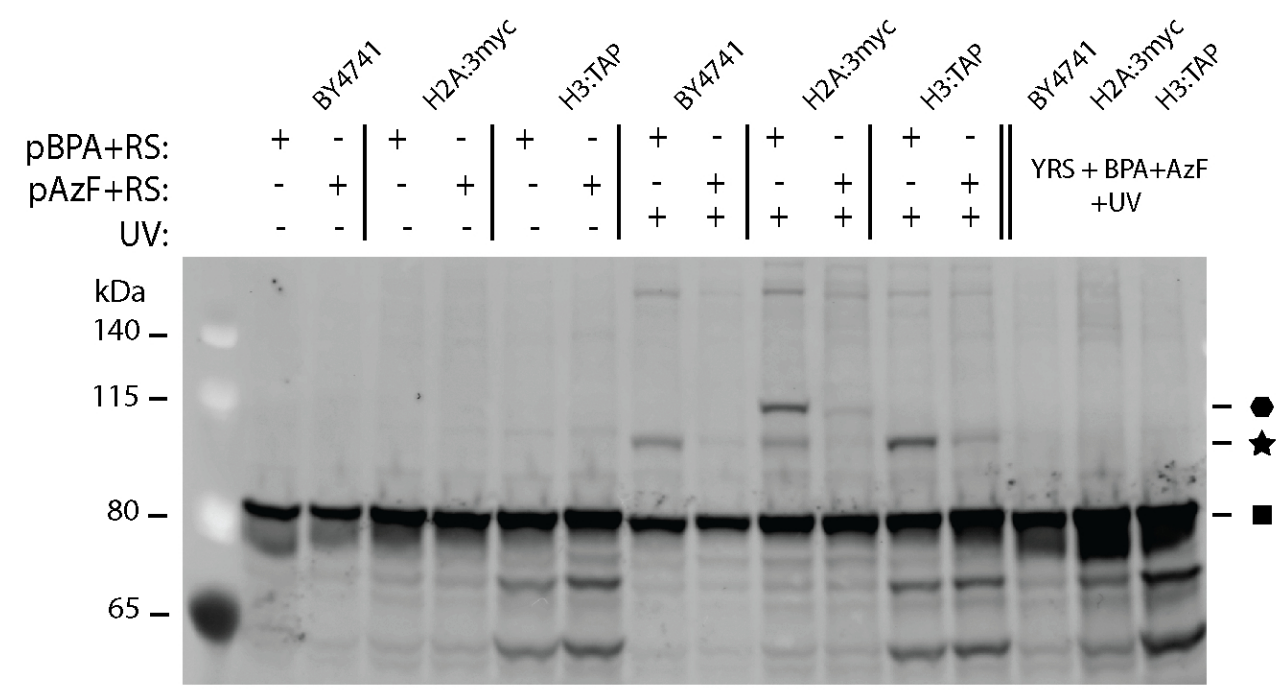

B.

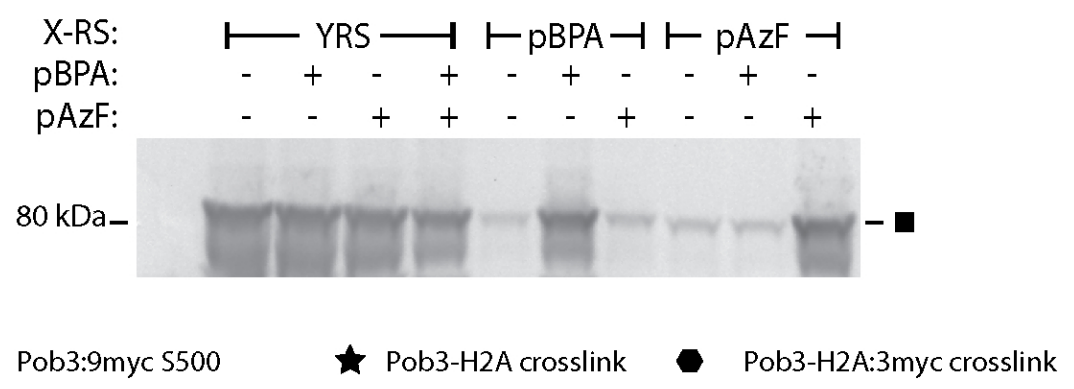

Figure 3.14: Investigation of the Pob3-S500 H2A crosslink comparing two different genetically encoded crosslinkers: 4-Benzoyl-L-phenylalanine (pBPA) and 4-Azido-L-phenylalanine $(\mathrm{pAzF})$ - A: Yeast strains containing the plasmid for incorporation of pBPA (pESC pBPARS LEU) or the plasmid for incorporation of $\mathrm{pAzF}$ (pESC pAzF-RS LEU) were transformed with plasmid p426 GAL Pob3:9mycS500TAG. The non-evolved tRNA-synthetase from E.coli (YRS) was used as control. Crosslinking cultures contained a mixture of $1 \%$ raffinose and $2 \%$ galactose as carbon source with either $1 \mathrm{mM} \mathrm{pBPA}$ or $2 \mathrm{mM} \mathrm{pAzF}$ or both. Cells were treated for 30 minutes with $365 \mathrm{~nm}$ light and protein extracts were prepared by TCA precipitation. Samples were analyzed by western blot (4-12 \% Bis-Tris gels; Hybond-LFP membrane, $800 \mathrm{mOD}_{600}$ per lane, $\alpha$-c-myc primary and Cy3-conjugated secondary antibody, Typhoon scan (Cy3-settings, PMT 400; $50 \mu \mathrm{m}$ pixel size; high sensitivity)). The crosslink formation is UV-dependent and crosslinker specific. The size shift due to a histone crosslink is observed only for histone H2A. Control experiments with cells containing the E.coli aminoacyl tRNA-synthetase for the incorporation of tyrosine (YRS) at Pob3-S500 shows no crosslink formation in the presence of a mixture of both unnatural amino acids in the media. B: BY4741 cells expressing Pob3:9myc-S500TAG in the presence of the YRS, pBPA or the pAzF ambers suppressor system were supplied with their cognate UAA or a mixture both. Cells were not subjected to UV light and total proteins were and analyzed by western blot against the myc-epitope. For detection the GE Typhoon FLA 7000 laser scanner was used (Green laser: 532 nm; long pass filter O580: $\geq 580 \mathrm{~nm}$; PTM 600; $25 \mu \mathrm{m}$ pixel size). Each amber suppressor system is specific for the incorporation of its evolved unnatural amino acid. 
a UV-dependent way. Interestingly, although the full-length protein comparing the pBPA- and the pAzF-system were equal, the crosslink intensity was generally lower in the pAzF samples. The Pob3 interaction with the histone H2A was confirmed in the $\mathrm{H} 2 \mathrm{~A}: 3$ myc strain seen in the molecular weight shift of the approximate size of the 3myc tag (5.0 kDa). A similar shift was also observed using the pAzF crosslinker. Hence, the formation of the interaction is independent of the crosslinker amino acid. A shift experiment in the H3:TAP strain resulted in no observable shift (TAP tag: approximately $20 \mathrm{kDa}$ ) indicating a specific interaction to $\mathrm{H} 2 \mathrm{~A}$ not $\mathrm{H} 3$ at serine 500 . Incorporating tyrosine at S500 using the non-evolved tRNA-synthetase from E.coli (YRS) resulted in full-length Pob3:9myc protein without any detectable crosslink formation (even in the presence of the unnatural amino acids pBPA and pAzF).

Furthermore, I tested the incorporation specificity of each specific evolved tRNA/ tRNA-synthetase pair (pBPA-RS and pAzF-RS) for their particular crosslinker amino acid (figure 3.14 B.). Full-length Pob3:9myc protein is only produced if cultures are supplemented with their respective unnatural amino acid. These cultures contain the specifically evolved tRNA/tRNA-synthetase pair for incorporation of pBPA or pAzF. In the absence of any or the cognate unnatural amino acid in the medium, the amount of produced protein is approximately an order of magnitude lower than in the presence of the cognate UAA and can therefore be neglected. In summary, the two tested systems are orthogonal in respect of their cognate unnatural amino acid.

I have successfully proven that the observed interaction between Pob3 at serine 500 and the histone H2A is independent of the used unnatural amino acid crosslinker. However, although the $\mathrm{pAzF}$ is structurally closer to an endogenous phenylalanine and smaller than the bulky pBPA. Nonetheless, I decided to prefer the UV-inducible crosslinker $\mathrm{pBPA}$ because the crosslink product yield is substantially higher compared to $\mathrm{pAzF}$ and cultures containing $\mathrm{pAzF}$ showed an up to approximately $50 \%$ slower growth than cultures containing $\mathrm{pBPA}$.

\subsubsection{Differential crosslinking pattern of the Pob3 CTD to the H2A-H2B dimer}

I found the first in vivo interaction of Pob3 at serine 500 with the histone H2A (section 3.2.1.1). In addition to the analyzed Pob3S500-H2A crosslink, my performed high-throughput interaction scan (figure 3.7) shows that experiments at neighboring positions result in crosslinks of similar size. Hence, I investigated the Pob3-CTD concerning histone interactions in more detail. I hypothesized that the CTD interacts with the histone H2A-H2B dimer. Therefore, I used two genomically 3:myc-tagged yeast strains to identify more histone-chaperone interactions (figure 3.15). 


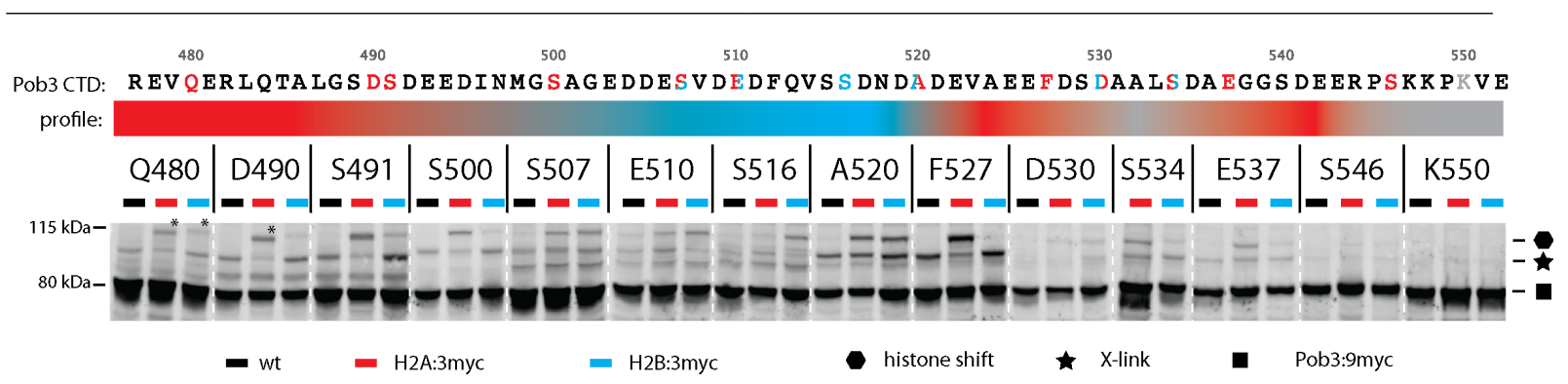

Figure 3.15: Scanning of the Pob3-CTD with pBPA for histone interactions in genomically tagged yeast strains - Yeast cells expressing Pob3:9myc with pBPA incorporated at 14 different positions of the CTD were subjected to $365 \mathrm{~nm}$ light for 20 minutes. Experiments were carried out in three different yeast strains: BY4741 (wild-type), BY4741 H2A:3myc and BY4741 H2B:3myc. Total proteins were extracted by TCA precipitation and analyzed by western blot (4-12 \% Bis-Tris Gels; Amersham Hybond-LFP membrane, $\alpha$-c-myc primary and Cy3-conjugated secondary antibody, Typhoon scan (Cy3-settings; PMT 400; $50 \mu \mathrm{m}$ pixel size; high sensitivity). A putative histone crosslink (star) can be observed at all tested sites except S546 and K550. The shift to a higher molecular weight in genomically-tagged strains is position and histone dependent (hexagon). The primary sequence of Pob3-CTD and the resulting color-coded interaction profile to histones $\mathrm{H} 2 \mathrm{~A}$ and $\mathrm{H} 2 \mathrm{~B}$ is depicted above the western blot.

Full-length Pob3:9myc protein was detected in similar levels at all tested positions (black square). Strikingly, in my molecular weight shift assay I see defined reproducible interactions of the Pob3-CTD with the histones $\mathrm{H} 2 \mathrm{~A}$ and $\mathrm{H} 2 \mathrm{~B}$ (hexagon). Apart from the very C-terminal positions, S546 and K550, I see crosslink formation for all other 12 tested positions. I observe interactions either strictly to H2A (e.g. D490, S500, F527), strictly to H2B (e.g. E510, S516) or to both histones (e.g. S507, A520). These experiments clearly indicate that the predicted structurally disordered acid C-terminal tail of Pob3 is forming specific and distinct interactions with the histones $\mathrm{H} 2 \mathrm{~A}$ and $\mathrm{H} 2 \mathrm{~B}$ in vivo.

These results motivated me to analyze the entire histone interaction network of the FACT complex and create a histone map. Since Spt16 also contains an acidic C-terminal tail, I performed a similar molecular weight shift assay with genomically tagged yeast strains for the Spt16-CTD (figure 3.16). As in analysis of Pob3-CTD, full-length Spt16:9myc protein could be detected in comparable levels at all tested positions (black square). With the exception of position Y972, no shift to a higher molecular weight at any position was detected. Although I was not expecting a drastic shift at this molecular weights (3:myc shift is roughly $5 \mathrm{kDa}$ ), a difference should be detectable. Only the at position Y972 I can observe a slight shift in a strain containing a genomically tagged $\mathrm{H} 2 \mathrm{~B}$ copy. Nonetheless, a characteristic distinct crosslinking behavior to the histones $\mathrm{H} 2 \mathrm{~A}$ and $\mathrm{H} 2 \mathrm{~B}$, as observed for the Pob3-CTD, can not be seen for the Spt16-CTD. 


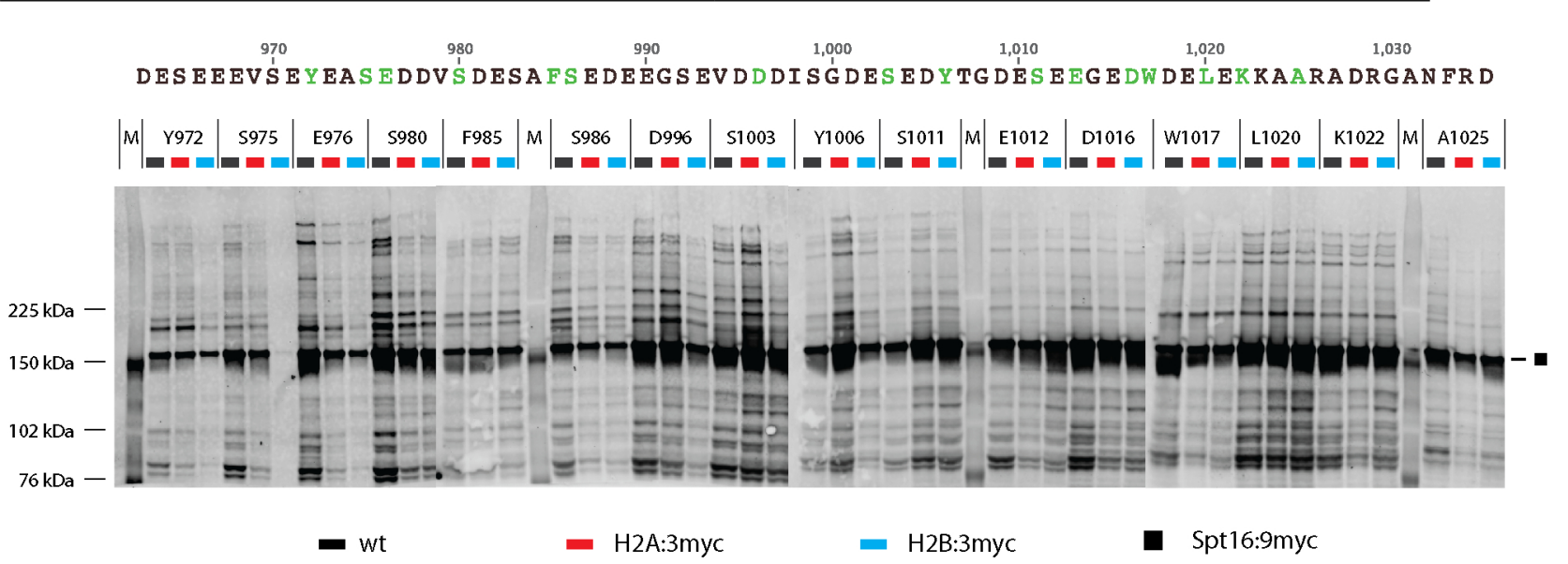

Figure 3.16: Scanning of the Spt16-CTD with pBPA for histone interactions in genomically tagged yeast strains - Yeast cells expressing Spt16:9myc with pBPA incorporated at 16 different amber sites of the CTD were subjected to $365 \mathrm{~nm}$ light for 30 minutes. Crosslinking time was extended due to experimental setup. Experiments were carried out in three different yeast strains: BY4741 (wt), BY4741 H2A:3myc and BY4741 H2B:3myc. Total proteins were extracted by TCA precipitation and analyzed by western blot (3-8\% Tris-Acetate gels; Amersham Hybond-LFP membrane, 500 mOD $_{600}$ per lane, $\alpha$-c-myc primary and Cy3conjugated secondary antibody, Typhoon scan (Cy3-settings)). Full-length Spt16:9myc protein can be observed at all tested sites except (S975 in BY4741 H2B:3myc). A slight shift to a higher molecular weight in a genomically-tagged H2B:3myc strains is observed at position Y972. The primary sequence of S.c. Spt16-CTD is shown above the western blot and pBPA positions are indicated in green.

\subsubsection{Interactions of the FACT complex to other histones}

The FACT complex is known to be an unusual histone chaperone since studies revealed interactions with histones $\mathrm{H} 2 \mathrm{~A} / \mathrm{H} 2 \mathrm{~B}$ and $\mathrm{H} 3 / \mathrm{H} 4$. Since I established molecular shift assays in genomically tagged histone strains, I wanted to analyze several distinct regions of the FACT complex in their histone binding behavior.

Analysis of the basic patch of the PH-domain of Pob3: Functional studies of the histone chaperone Rtt106 revealed a basic patch (Rtt106 S80-R86) on the N-terminal $\mathrm{PH}$ domain which is crucial for $\mathrm{H} 3 / \mathrm{H} 4$ binding 177 . The PH domains of Pob3 and Rtt106 can be superimposed and share the same overall structure. Since the Rtt106 model proposes that two distinct binding surfaces are present on Rtt106 and the second motif (Rtt106: 264-268; ITRLT-loop) was clarified to determine the specificity of Rtt106 for H3, I tested the first motif (basic patch) of Pob3 for interactions to histone H4 instead of H3. The residues F250-R256 are structurally homologue to the Rtt106 basic patch S80-R86 (pdb Pob3: 2GCL, Rtt106: 3TVV)

Crosslinking experiments were carried out in wild-type BY4741 and genomically H4:3myc-tagged yeast strains. The crosslinker was incorporated at 14 different sites of the Pob3-M domain ranging from K190 to L300 (figure 3.17). Full length Pob3:9myc 


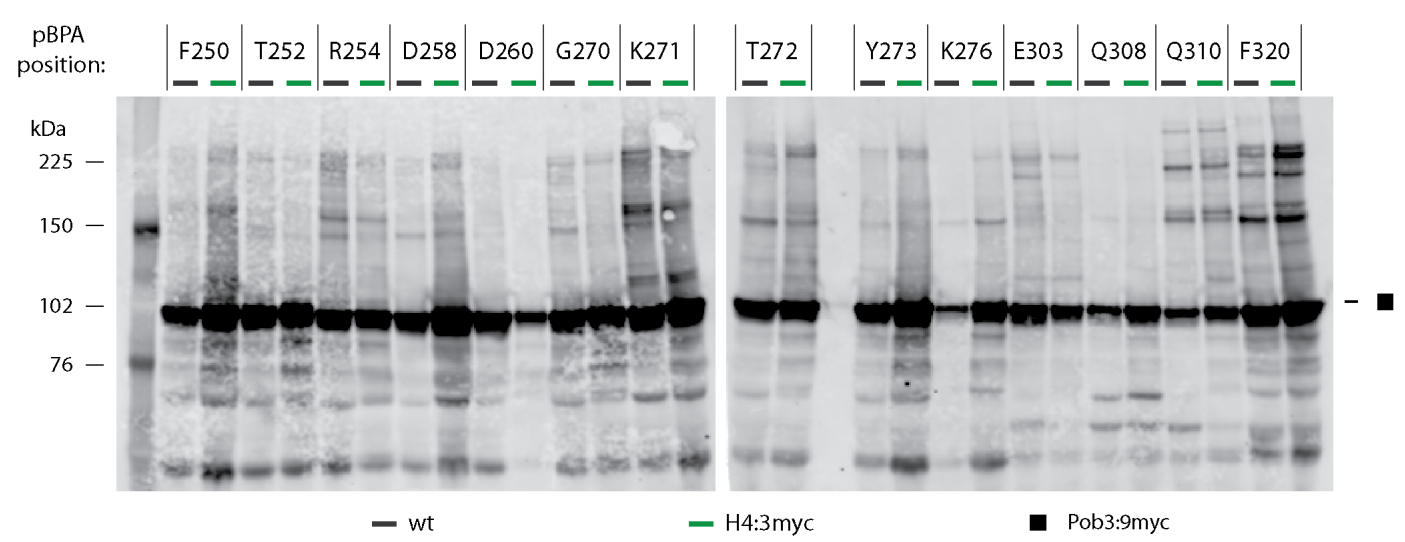

Figure 3.17: Scanning of the Pob3-MD basic batch with pBPA for $\mathrm{H} 4$ interactions using a genomically tagged H4:3myc yeast strain - Yeast cells expressing Pob3:9myc with pBPA incorporated at 14 different sites of the Pob3M domain were subjected to $365 \mathrm{~nm}$ light. Experiments were carried out in two different yeast strains: BY4741 (wild-type) and BY4741 H4:3myc. Total proteins were extracted by TCA precipitation and analysed by western blot (3-8\% Tris-Acetate Gels; Hybond-LFP membrane, $\alpha$-c-myc primary and Cy3-conjugated secondary antibody, Typhoon scan (Cy3-settings, PMT 400; $200 \mu \mathrm{m}$ pixel size)). Full-length Pob3:9myc protein is observed at all tested sites. A shift to a higher molecular weight in genomically tagged H4:3myc strain samples could not be identified.

protein was detected at all sites and strain combinations. The crosslinks of higher molecular weight show similar patterns as the ones detected in the high-throughput screen of Pob3. This indicates the high reproducibility of the crosslink approach (compare figure 3.7, e.g. K271, T272, Q310 and F320). However, no observable shift to a higher molecular weight in samples of the genomically H4:3myc-tagged yeast strain are detected. Thus, I conclude no detectable interactions at these positions with $\mathrm{H} 4$ in vivo.

Interactions of the Spt16 $\mathrm{N}$-terminal with histone $\mathrm{H} 3 / \mathrm{H} 4$ : As part of the FACT complex, Spt16 is known to interact with H2A-H2B and H3-H4 in vitro. However, direct in vivo interactions are still poorly understood. Of particular interest is the Spt16-NTD sharing a "pita-bread" fold which is found in several amino peptidases 111 . Size exclusion chromatography using the isolated Spt16-NTD from Schizosaccharomyces pombe revealed binding to histone $\mathrm{H} 3-\mathrm{H} 4{ }^{174}$. However a complete deletion of the NTD in Saccharomyces cerevisiae is not detrimental unless not combined with a mutation in the FACT complex subunit Pob3 111 . To gain a deeper understanding of these complex interactions in the natural context, I analyzed the putative implicated H3-H4 binding region with my developed crosslinking approach (homologues in S.c.Pob3: S77-K80 and D268-S272/F372/F373). The high-througput screen shows no interesting putative histone crosslinks at the positions S78 and K80 (figure 3.7). However several residues, such as K108, K142, D251, show an additional putative 
histone crosslink by a protein band increased only by only a few kilodaltons. Interestingly, all these residues point are surface exposed and point in the same direction of the putative histone interaction surface (figure 3.18 A.). I performed crosslinking

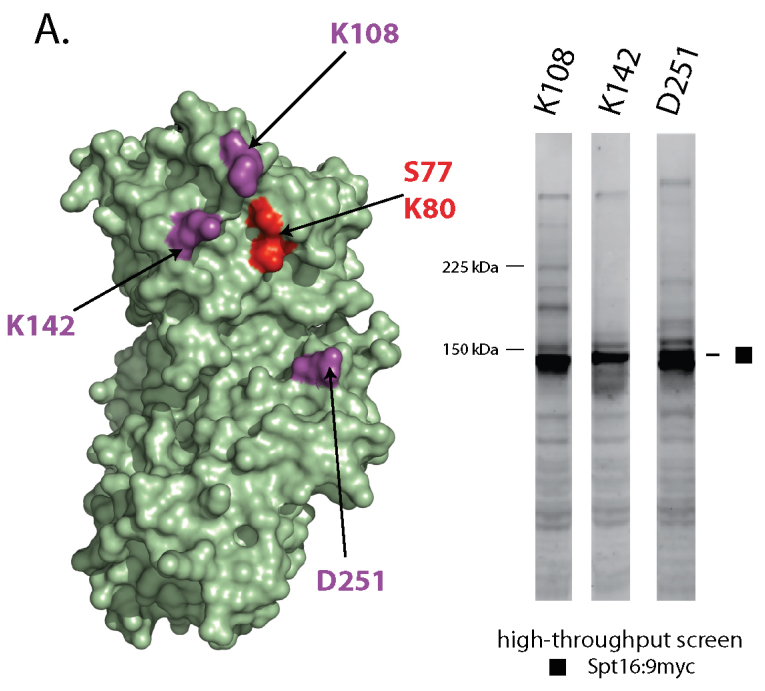

B. Molecular weight shift assays:

Figure 3.18: Molecular weight shift experiments of the Spt16-NTD with genomically tagged yeast strains - A: Spt16-NDT (pdb: 3BIQ) shown as surface model. Putative H4-interaction sites in red. Interesting crosslink sites depicted from high-throughput scan experiment (figure 3.7) and shown in violet. B: Yeast cells expressing Spt16:9myc with pBPA incorporated at 3 different amber sites of the Spt16-NTD were subjected to $365 \mathrm{~nm}$ light. Experiments were carried out in three different yeast strains: BY4741 (wild-type), H3:TAP and H4:GFP. Total proteins were extracted by TCA precipitation and analysed by western blot (3$8 \%$ Tris-Acetate Gels; Hybond-LFP membrane, $\alpha$-c-myc primary and Cy3-conjugated secondary antibody, Typhoon scan (Cy3-settings; PMT 400; $50 \mu \mathrm{m}$ pixel size))

experiments at the sites K108, K142 and D251 of Spt16-NTD in three different yeast strains. For the shift assays strains with genomically tagged H3 with the TAP tag and H4 with a GFP tag were used. Although the crosslinking pattern was reproducible for the higher molecular weights, the interesting putative crosslink was not observed. This might be due decreased separation distance of the Tris-Acetate-PAGE gel. Nevertheless, no distinct molecular weight shifts of crosslinked products by the size of a TAP ( $\sim 20 \mathrm{kDa})$ or GFP-tag $(\sim 25 \mathrm{kDa})$ at the same sites were observed. Hence, I could not detect a H3 or H4 interaction at the tested site of Spt16-NTD.

Interactions of the Spt16-M domain with the four core histones: Similar to the Pob3-MD, the middle domain of Spt16 consist of a double PH motif followed by the acidic C-terminal domain. Superimposition of these middle domain structures results in root mean square deviations (r.m.s.d.) of $1.7 \AA$ over 156 residues $\frac{176}{17}$. This structural homology implies binding to histones such are the ones for the PH domain of Rtt106. After testing the Pob3 middle domain and the Spt16-NTD, I analyzed the Spt16-MD domain for putative histone interactions. I performed crosslink experiments at 10 
different positions of the Spt16 middle domain. For molecular weight shifts, I used GFP-tagged strains, such as H2A:GFP, H2B:GFP and H4:GFP, and the H3:TAP strain (figure 3.19). For comparison, the sites from the high-throughput screen are depicted in part A. The shift experiment was split in two parts: In the first part (B) the sites L791, D908, S936 and Y942 were tested with H3:TAP and H4:GFP strains and in the second part (C) the sites, F878, K881, V888, D908, Q938, W950 and S986 were tested against all four core histone.

A. pBPA-screen crosslinks :

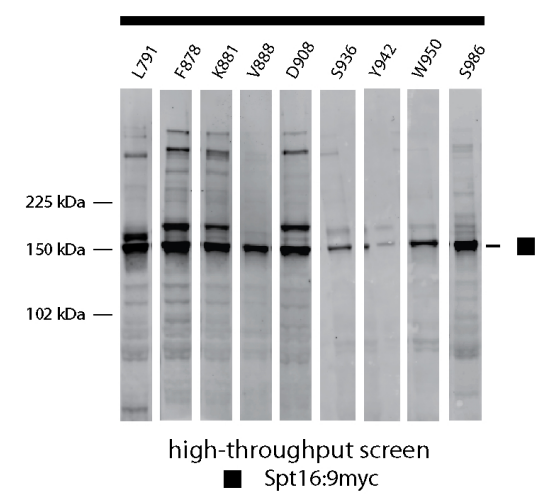

B. Shift assays Spt16-M (I.)

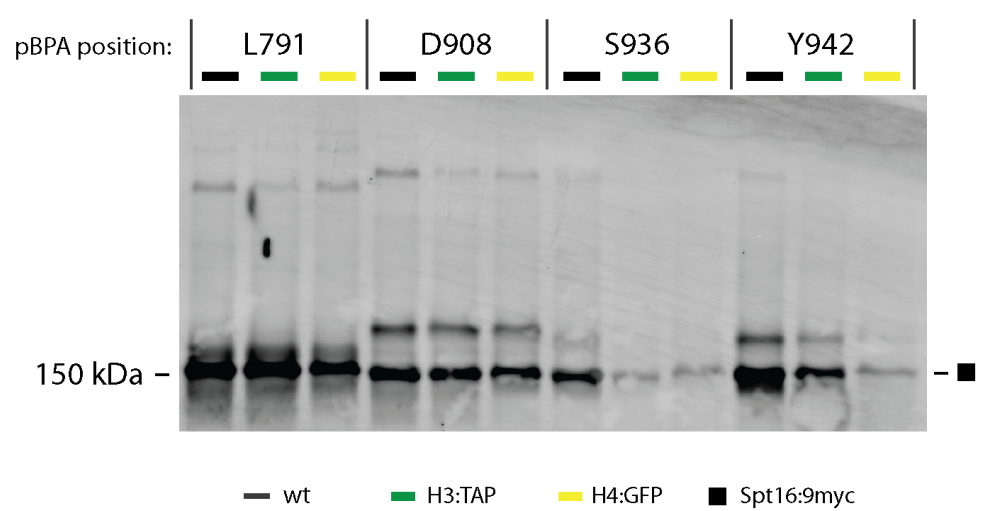

C. Shift assays Spt 16-M (II.)
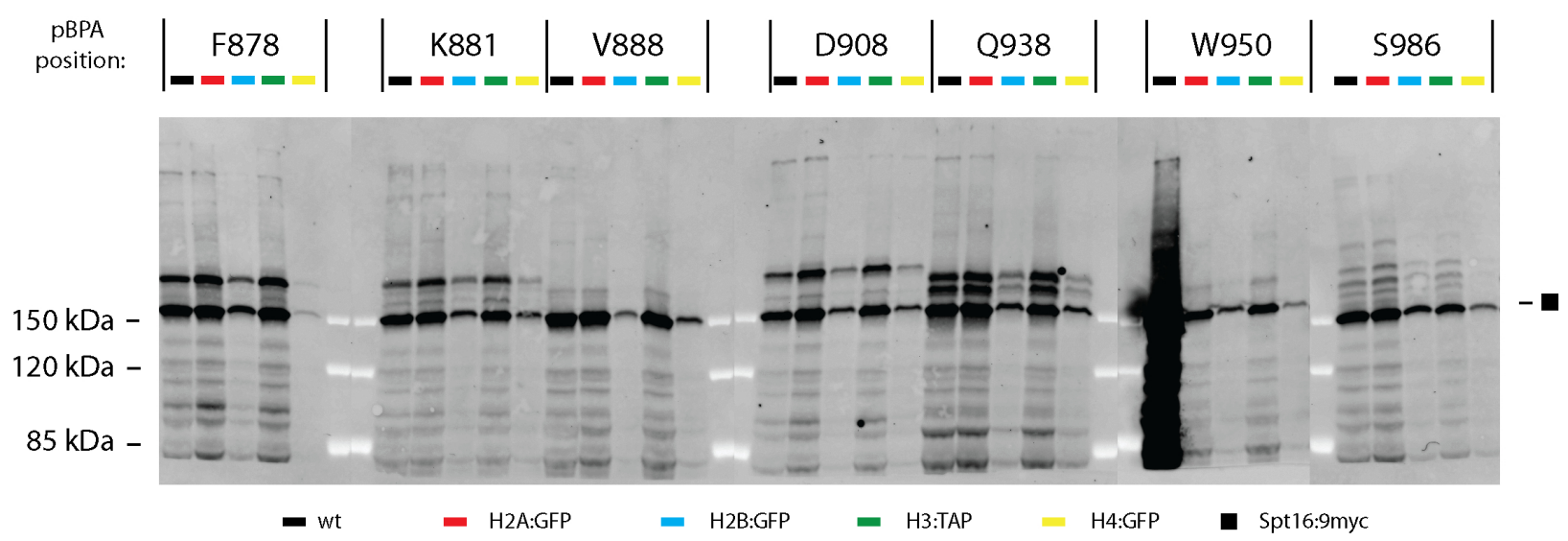

Figure 3.19: Molecular weight shift experiments of the Spt16 middle domain using genomically tagged yeast strains - A: Interesting putative histone crosslink sites depicted from highthroughput scan experiment (figure 3.7). B/C: Yeast cells expressing Spt16:9myc with pBPA incorporated at 4/7 different amber sites of the Spt16-NTD were subjected to 365 $\mathrm{nm}$ light. Experiments were carried out in four different yeast strains: BY4741 (wild-type), H2A:GFP, H2B:GFP, H3:TAP and H4:GFP (for B: H3:TAP and H4:GFP only). Total proteins were extracted by TCA precipitation and analyzed by western blot (3-8\% Tris-Acetate gels; Amersham Hybond-LFP membrane, $\alpha$-c-myc primary and Cy3-conjugated secondary antibody, Typhoon scan (Cy3-settings; PTM 400; $50 \mu \mathrm{m}$ pixel size)). 
Together, I was able to reproduce the crosslinking pattern that I observed in the pBPA high-throughput screen (figure 3.7). The interesting crosslinked products are indicated by a strong protein signal of slightly higher molecular weight than the full-length Spt16:9myc protein (e.g. F878, K881, D908 and Q938). Protein levels of full-length Spt16:9myc protein were generally lower in samples of the H2B:GFP and H4:GFP compared to the other tested strains. However, none of the examined strong crosslinked products shows a shift to a higher molecular weight in a genomicallytagged yeast strain. Hence, they were no strong histone interactions at these tested sites with the pBPA crosslinking method in vivo.

\subsubsection{Immunoprecipitation of pBPA crosslink adducts}

In addition to the molecular weight shift approach, I wanted to perform an unbiased identification approach without a putative candidate prescreening. Therefore, I immunoprecipitated the crosslinked products for subsequent identification by mass spectrometry. I chose the Pob3:S500 position as the test position to optimize the immunoprecipitation method. Therefore, I used a strain expressing Pob3:9myc with pBPA at serine 500 and immunoprecipitated the Pob3:9myc by anti-myc-antibodies coupled to protein G Dynabeads ${ }^{\mathrm{TM}}$. Samples were analyzed western blot against the myc-epitope (figure 3.20 A).

The WCE control samples show an UV-dependent crosslink product formation (black square and star). Hence, the analyzed IP samples were of good initial quality. The samples of flow-through, the non-bound material, still contain Pob3:9myc full-length protein indicating that the beads were saturated with the sample. Furthermore, the loss of the Pob3:9myc signal in the wash samples indicates a sufficient cleaning of the beads. In the non-UV-treated control, the elution samples contain only the Pob3:9myc protein, whereas the elution of the UV-treated samples show the presence of the Pob3:H2A crosslink product (star). This western blot shows that the principle of the immunoprecipitation of Pob3:9myc with antibody-coupled protein G Dynabeads $^{\mathrm{TM}}$ is successfully applied to the crosslink samples. The residual eluate was loaded on a 4-12\% Bis-Tris gel and stained with InstantBlue ${ }^{\mathrm{TM}}$, a single step Coomassie based gel stain. The gel shows two prominent bands at $65 \mathrm{kDa}$ and $50 \mathrm{kDa}$. The $50 \mathrm{kDa}$ band can be assigned to the heavy chain of the anti-myc-antibody and the band at $65 \mathrm{kDa}$ might be BSA which bound unspecifically during the antibody coupling reaction. Alternatively, these could represent the truncation products of the Pob3:9myc protein. However, in the contrast enhanced view of the gel, I see some faint bands at the expected size of the Pob3:9myc and the respective crosslink can be detected. Furthermore, the presence of the crosslink product seems not be not 
UV-dependent. One reason for the high abundance of Pob3:9myc and the crosslink might be due to the low amount of antibody which was coupled to the beads $(5 \mu \mathrm{g}$ per $40 \mu \mathrm{L}$ beads). The binding capacity of the Dynabeads ${ }^{\mathrm{TM}}$ is given with up to $25 \mu \mathrm{g}$ mouse IgG per $40 \mu \mathrm{L}$ beads. Altogether, the principle of the immunoprecipitation could be successfully applied to the Pob3:9myc crosslink samples indicated by western blot. However, for further identification by mass spectrometry, the protocol needs to be optimized.

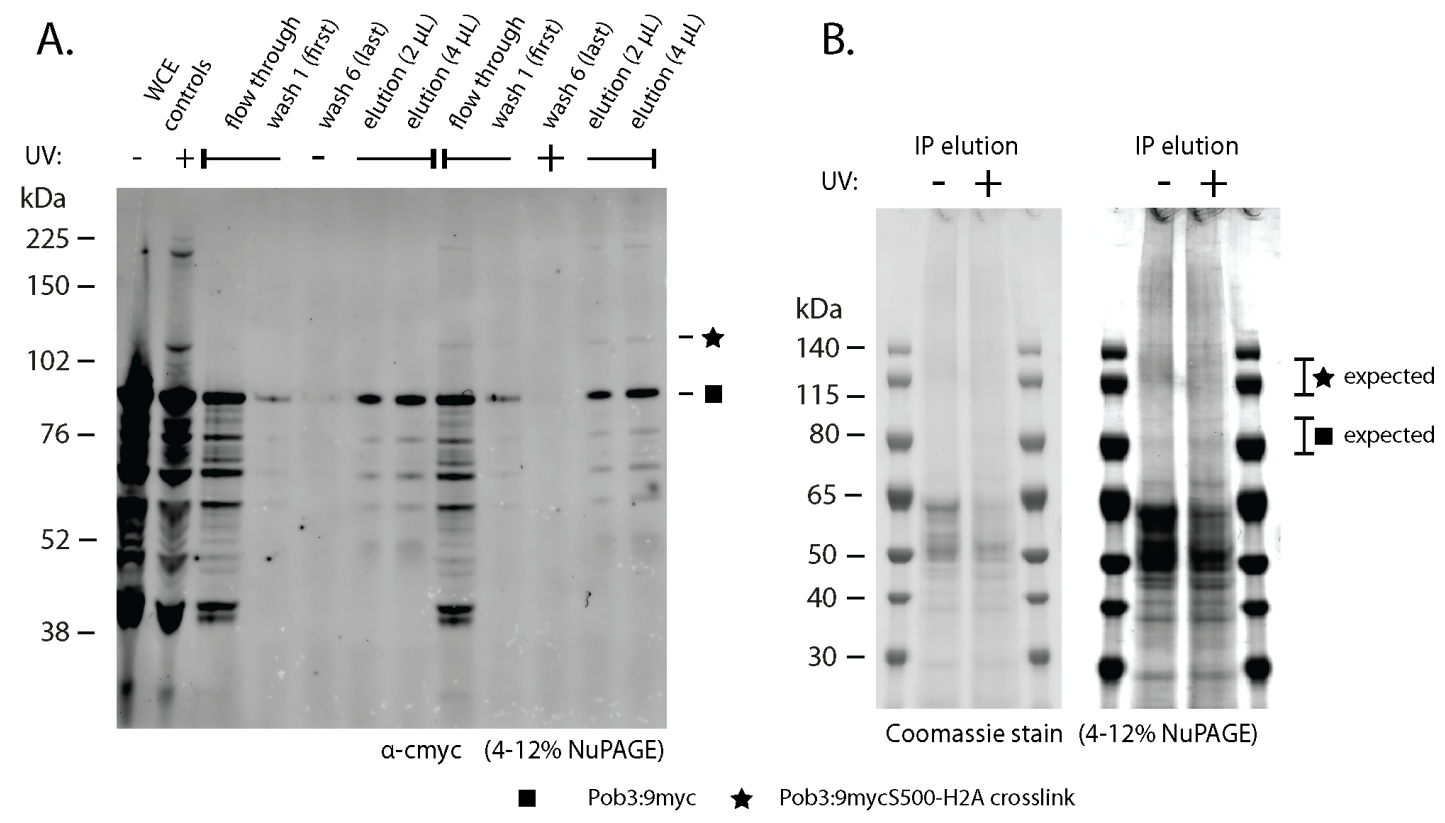

Figure 3.20: Immunoprecipitation of the Pob3:9mycS500-H2A crosslink analyzed by western blot and SDS-PAGE - A: In vivo pBPA crosslinking experiments were performed in BY4741 cells using the Pob3:9mycS500TAG construct. Crosslink samples (15 minutes irradiation) and non-UV-treated control $\left(360 \mathrm{OD}_{600}\right.$ each) were subjected to immunoprecipitation as described in 2.2.2.8. The flow-through sample represents the non-bound lysate sample after incubation with the beads $(10 \mu \mathrm{L})$. Whole cell extract controls $\left(12 \mathrm{OD}_{600}\right)$ were taken before harvesting the IP cultures. The control was treated for 7 minutes with UV-light and subjected to standard TCA precipitation $\left(600 \mathrm{mOD}_{600}\right.$ per lane). All samples were analyzed by western blot against the myc-epitope (4-12 \% Bis-Tris Gels; Amersham Hybond-LFP membrane, $\alpha$-c-myc primary and Cy3-conjugated secondary antibody, Typhoon scan (Cy3-settings; PMT 450; $50 \mu \mathrm{m}$ pixel size)). B: The residual IP eluate was loaded on a 4-12\% Bis-Tris gel (1 x MOPS buffer) and stained with InstantBlue ${ }^{\mathrm{TM}}$.

\subsection{In vitro analysis of FACT interactions using the complex homologue from Chaetomium thermophilum}

This section contains the investigations of the Pob3-histone interactions in a defined isolated system. I wanted to confirm the observed in vivo interactions by a pBPA 
crosslinking approach in a more defined surrounding, hence in vitro. I used the Pob3-homologue protein from Chaetomium thermophilum (ctPob3) and purified it from Escherichia coli. For crosslink experiments, I applied the Genetic code expansion system for incorporation of the unnatural UV-inducible crosslinker pBPA in bacteria 2601297. I tested several positions of the ctPob3 for interactions with reconstituted Xenopus laevis H2A-H2B histone dimers, H3-H4 tetramers or with reconstituted mono nucleosomes. Finally, I cloned and purified the Nhp6a protein from Saccharomyces cerevisiae for subsequent crosslink assays.

\subsubsection{Expression of ctPob3 containing the genetically encoded UV-inducible crosslinker pBPA in Escherichia coli}

The bacterial expression plasmid pRSF His-TEV-ctPob3 was subjected to Quikchange PCR mutagenesis. Amber TAG-codons were introduced at 10 different positions throughout the open reading frame. The positions were selected using a protein sequence alignment of the Saccharomyces and Chaetomium Pob3 sequence supported by structural data and the pBPA screen (figure 3.21A and B).

The proteins share a pairwise identity of $42.2 \%$ with 242 identical sites. The expression of ctPob3-pBPA mutants was analyzed by western blot against the Hisepitope (exemplary figure 3.21 C. for pBPA incorporation at S496). The western blot shows efficient expression of His-TEV-ctPob3 protein only in the presence of the unnatural amino acid pBPA after 3 hours of expression (last lane). Although, full-length protein is already observed in small amounts before induction, the protein level did not increase after 3 hours of expression in the absence of pBPA. Hence, the increased protein levels in the presence of pBPA after 3 hours indicate successful expression of mutant ctPob3-pBPA proteins in Escherichia coli. Proteins were purified from the expression cultures by Ni-NTA affinity chromatography and used for subsequent in vitro crosslinking experiments. Total yields of up to $9 \mathrm{mg}$ protein after NiNTA elution per liter expression culture were obtained.

\subsubsection{In vitro crosslinking studies of ctPob3-pBPA to reconstituted histone dimers and tetramers}

Xenopus laevis $\mathrm{H} 2 \mathrm{~A}-\mathrm{H} 2 \mathrm{~B}$ histone dimers and $\mathrm{H} 3-\mathrm{H} 4$ tetramers were reconstituted as previously described in the material and methods part (2.2.3.1). A chromatogram of the analytical gel filtration runs and their SDS-PAGE analysis is shown in figure 3.22 .

The elution profiles of the reconstituted histone dimers and tetramers are consistent with reported literature ${ }^{80}$. Each reconstitution resulted in one major peak. The H2A- 
A. Protein alignment of Pob3 from Saccharomyces cerevisiae and Chaetomium thermophilum

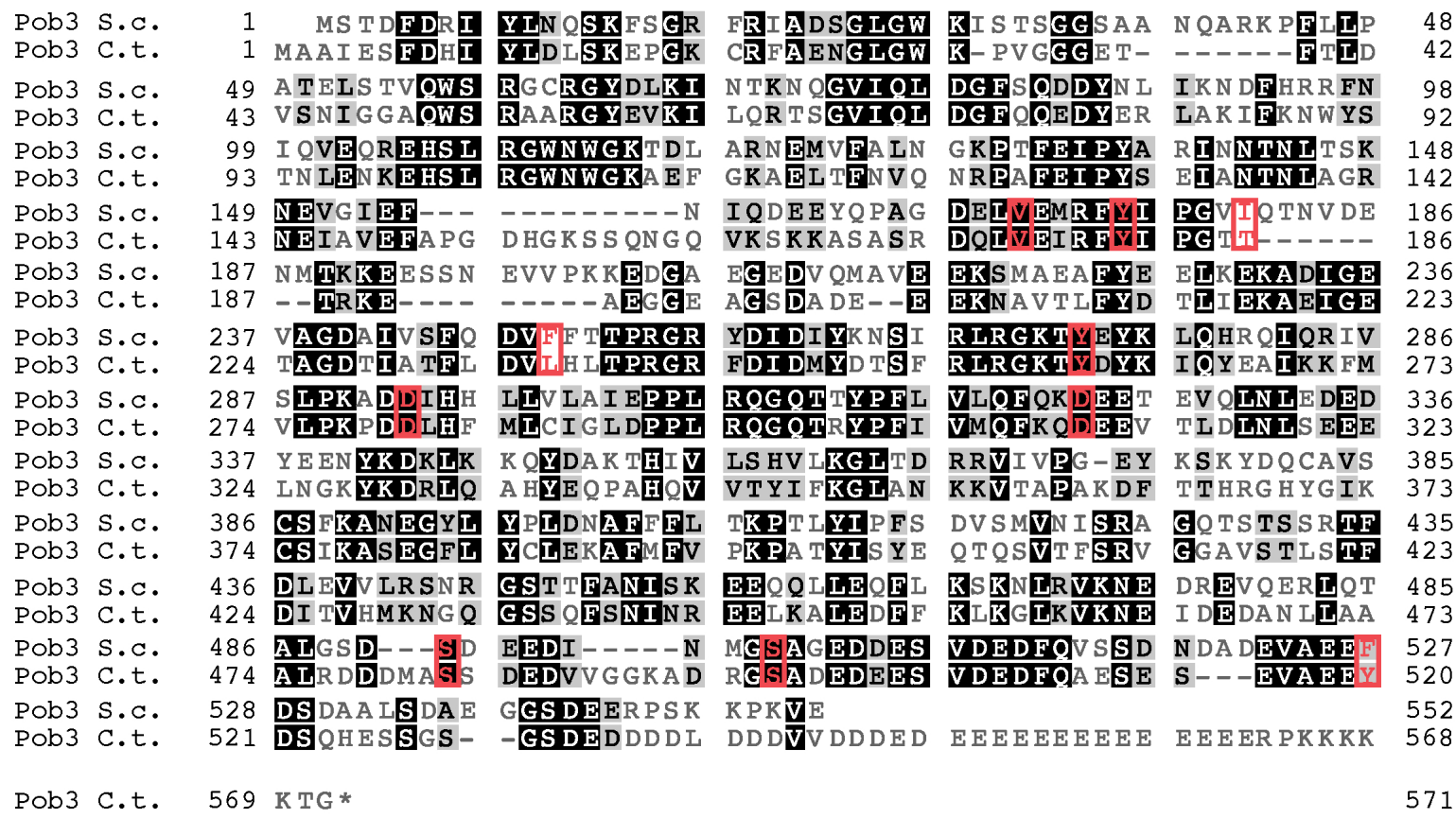

B. Crystal structure of S.c. Pob3-MD

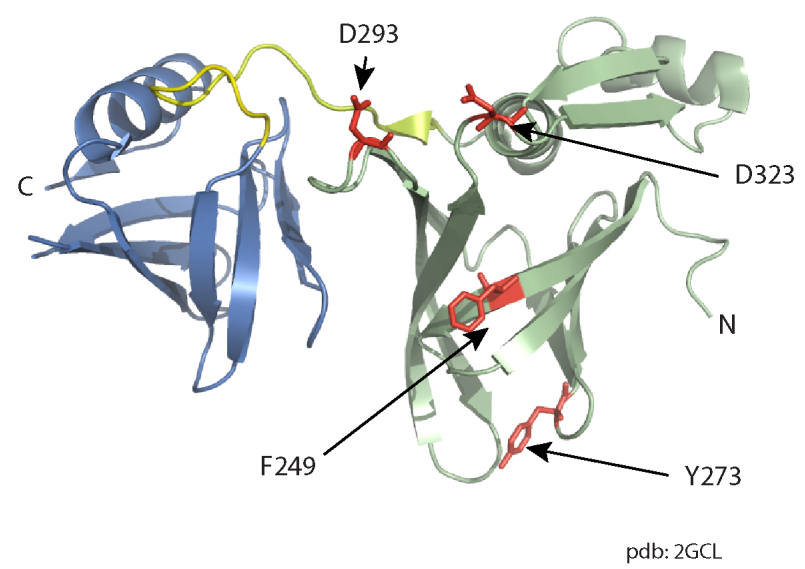

C. Expression of ctPob3pBPA mutant in E.coli

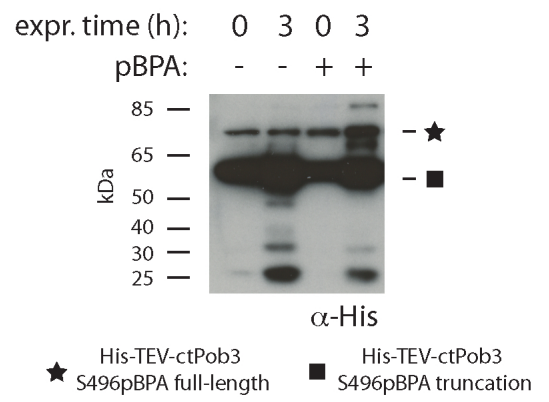

Figure 3.21: Protein alignment of Saccharomyces cerevisiae and Chaetomium thermophilum Pob3 and western blot of ctPob3:S496BPA expression - A: Protein sequence alignment of Pob3 from Saccharomyces cerevisiae and Chaetomium thermophilum (matrix: Blosum62; gap penalty: 12; gap extension penalty: 3; global alignment with free end gaps; background similarity color code: identical (black), similar (grey, Blosum62 threshold: 1), not similar (white)). Sites used for in vitro ctPob3 crosslinking are depicted in red. B: Crystal structure as ribbon diagram of the Pob3-MD (pdb: 2GCL). PH1 domain (green), PH2 domain (blue), linker (yellow). pBPA-in vitro-crosslink positions in red. C: Western blot of His-TEVctPob3 expression with incorporated pBPA at S496. E.coli BL21 cells were transformed with plasmid pSUP pBPA and plasmid pRSF His-TEV-ctPob3S496TAG. Expression was performed with or without $1 \mathrm{mM}$ BPA and induced with $1 \mathrm{mM}$ IPTG. Samples were taken at indicated time points and boiled in $1 x$ Sample Buffer at $95^{\circ} \mathrm{C}$ for 10 minutes. Samples were analyzed by SDS-PAGE and western blot against the his-tag-epitope (4-12 \% Bis-Tris gel; Amersham Hybond-LFP membrane, $70 \mathrm{mOD}_{600}$ per lane). The detection was done with the Amersham ECL Plus ${ }^{\mathrm{TM}}$ Western Blotting Detection Reagents and Amersham Hyperfilm ECL (3 seconds exposure). 
$\mathrm{H} 2 \mathrm{~B}$ reconstitution peak had an absorbance of approximately $770 \mathrm{mAU}$ and eluted at $15.2 \mathrm{~mL}$. The corresponding fractions (B11 to B8) showed a protein signal at the expected molecular weight for H2A and H2B (14 kDa). Due to their similar size, histone H2A and H2B run as one band on the $15 \%$ SDS-PAGE gel. Since equal amounts of each histone for each reconstitution was used, the signal intensity can be used as confirmation. The approximately doubled intensity of the H2A/H2B dimer band signal (black hexagon) compared to the single $\mathrm{H} 3$ and $\mathrm{H} 4$ protein bands indicates the presence of both proteins $\mathrm{H} 2 \mathrm{~A}$ and $\mathrm{H} 2 \mathrm{~B}$ (additive effect of $\mathrm{H} 3$ and H4; black star and square). The chromatogram for the H3-H4 tetramer reconstitution revealed one major peak which had an absorbance of approximately $330 \mathrm{mAU}$ and eluted at $13.3 \mathrm{~mL}$. The corresponding fractions (A10 to B12) showed two protein signals at the expected molecular weight for $\mathrm{H} 3$ and $\mathrm{H} 4$ (15.3 kDa and $11.4 \mathrm{kDa})$. The coelution of the histones in both reconstitution indicated successful refolding of the dimers and tetramers.

The reconstituted histone $\mathrm{H} 2 \mathrm{~A}-\mathrm{H} 2 \mathrm{~B}$ dimers were combined with the purified ctPob3-pBPA mutant proteins in an in vitro crosslink experiment (for pBPA sites see figure 3.21 (A.), sites in red). CtPob3-pBPA mutant proteins were incubated with a one molar excess of $\mathrm{H} 2 \mathrm{~A}-\mathrm{H} 2 \mathrm{~B}$ dimers and crosslinked. Protein concentrations were determined with Bradford. Due to the vast amount of protein truncation at the amber suppressor codon, the protein amount of full-length Pob3-pBPA protein was estimated to be approximately a tenth of the total protein amount of the sample. Nevertheless, the following calculations and ratios are normalized to the total protein amount of Pob3-pBPA mutants, thereby neglecting this ten-fold shortage. For controls, samples aliquots were taken prior to UV-treatment. Samples were analyzed by SDS-PAGE and either stained with InstantBlue ${ }^{\mathrm{TM}}$ or subjected to western blot against the his-tag-epitope. The stained gels show a defined full-length ctPob3BPA protein signal with a molecular weight of approximately $70 \mathrm{kDa}$ (black square, figure 3.22 (B.) top). An UV-dependent shift to a higher molecular weight of this band in the UV-treated samples might indicate an interaction between ctPob3pBPA and the histone H2A-H2B dimer. Experiments show defined reproducible shifts at position D280, D310, S482, S496 and Y520. The additional weight shift is directly connected with a decrease of the full-length ctPob3pBPA protein signal indicating a crosslink formation of the ctPob3pBPA protein. The western blot analysis was performed to detect the ctPob3pBPA proteins since they contain a N-terminal His-TEV-tag. The same UV-dependent shift pattern is revealed on the western blot confirming that shifts originate from ctPob3pBPA mutant proteins. A control with the wild-type ctPob3 protein shows no shifts to a higher molecular weight upon treatment with UV-light in both analyses. 
A. Chromatogram and SDS-PAGE analysis from a S200 size exclusion chromatography of reconsituted Xenopus laevis histone dimers and tetramers

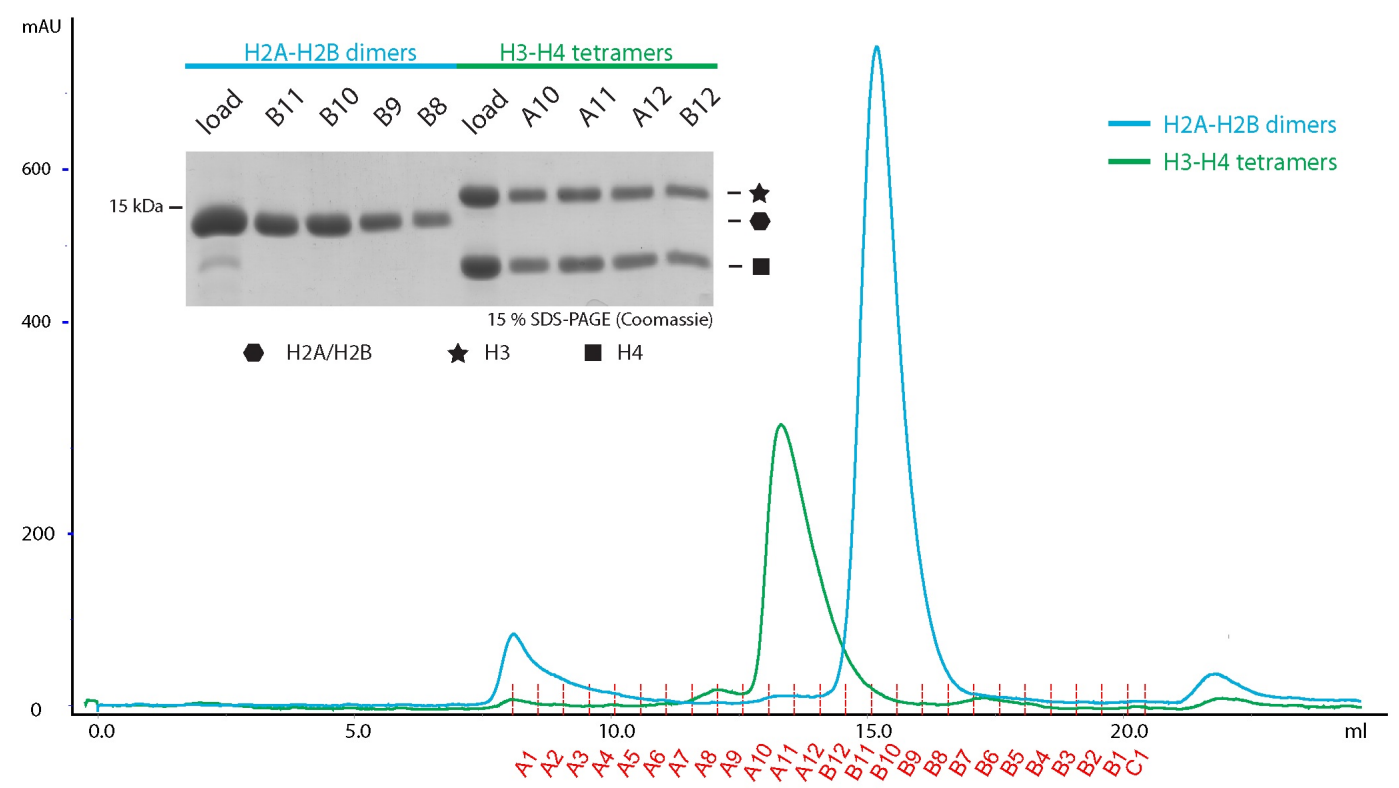

B. In vitro crosslink analysis of ctPob3 pBPA mutants with histone $\mathrm{H} 2 \mathrm{~A}-\mathrm{H} 2 \mathrm{~B}$ dimers

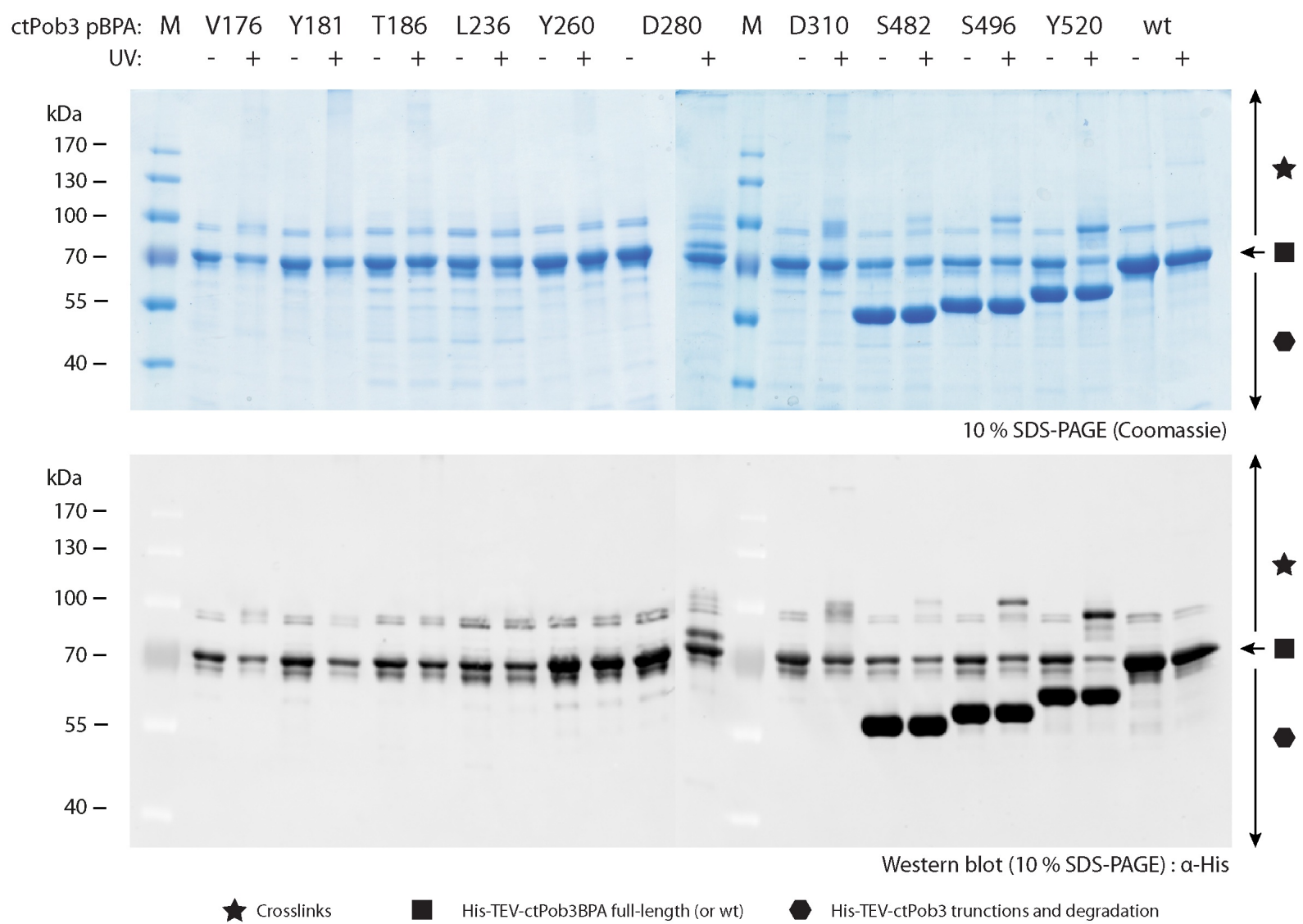

Figure 3.22: Size exclusion chromatography analysis of reconstituted Xenopus laevis histone dimers and tetramers and in vitro crosslink analysis of ctPob3 pBPA mutants with histone H2A-H2B dimers Caption next page. 
(Previous page.) A: Size exclusion chromatography elution profile and SDS-PAGE analysis of reconstituted Xenopus laevis histone dimers and tetramers. A mixture of either histone H2A and H2B (blue) or histone $\mathrm{H} 3$ and $\mathrm{H} 4$ (green) were taken for reconstitution (2.2.3.1). A small volume (3 $\mu \mathrm{L})$ of selected fractions from the size exclusion chromatography was analyzed by SDS-PAGE (15\% gel, InstantBlue ${ }^{\text {TM }}$ stain, load: gelfiltration loading sample). B: In vitro crosslink analysis of ctPob3 pBPA mutants with histone H2A-H2B dimers. Reactions of different His-TEV-ctPob3-BPA mutant proteins (total mass) with a one molar excess of histone $\mathrm{H} 2 \mathrm{~A}-\mathrm{H} 2 \mathrm{~B}$ dimer were prepared and half of the reactions were subjected to $365 \mathrm{~nm}$ light for 45 minutes on ice. Treated and non-treated samples were boiled for 5 minutes at $95^{\circ} \mathrm{C}$ and analyzed by $10 \%$ SDS-PAGE (reaction per lane: $5 \mu \mathrm{g}$ ctPob3-BPA mutant (NiNTA purified) with $3.9 \mu \mathrm{g} \mathrm{H}$ A/H2B dimer, final buffer: $50 \mathrm{mM}$ TrisHCl pH7.5, $200 \mathrm{mM} \mathrm{NaCl}$, $1 \mathrm{mM}$ EDTA, $1 \mathrm{mM}$ DTT, $100 \mathrm{mM}$ imidazole). Gels were prepared in duplicates whereas one was stained with InstantBlue $^{\mathrm{TM}}$ and the other subjected to western blot analysis (Amersham Hybond LFP, $\alpha$-HIS primary and Cy3-conjugated secondary antibody, Typhoon scan (Cy3-settings; PMT 350; $200 \mu \mathrm{m}$ pixel size)).

To exclude crosslinking artifacts of the ctPob3pBPA mutants in the absence of the histone dimer, I performed a titration series of H2A-H2B dimer with the ctPob3S496pBPA mutant (figure 3.23). Increasing amounts of histone H2A-H2B dimers were added to the ctPob3S496pBPA mutant and crosslinked for 45 minutes on ice. The concentrations ranged from one molar excess $(7 \mu \mathrm{M})$ to ten molar shortage $(0.35 \mu \mathrm{M})$ of the histone dimer to the ctPob3pBPA mutant protein. The Coomassie-stained gel shows comparable amounts of the full-length His-TEV-ctPob3pBPA protein and the truncation in each sample (figure 3.23 left, black square and hexagon). The sample which contains a two molar excess of the histone dimer to the Pob3pBPA mutant shows the previously observed crosslink (star, compare figure 3.22 B.). Decreasing the amount of histone dimer while keeping the experimental setup constant resulted in a concentration-dependent decrease of the crosslink signal up to the detection limit of the Coomassie stain (molar ratio: 0.1:1). The western blot against the his-tag-epitope, visualizing the His-TEV-ctPob3S496 mutant proteins, confirm the concentration-dependent crosslink formation. Hence, the observed crosslink is dependent on the presence and furthermore concentration of the histone dimer. Additionally, UV-treatment of the ctPob3S496BPA mutant in the presence of low amounts of histone dimer $(0.35 \mu \mathrm{M})$ showed no crosslink formation. Hence, the crosslink formation is not an artifact of the pBPA-mutant itself. Similar experiments were performed using the $\mathrm{H} 3-\mathrm{H} 4$ tetramer for crosslink analysis with ctPob3-pBPA mutants. However, I observed visible white precipitation upon incubation of the Pob3pBPA mutants with the H3-H4 tetramer. Therefore, I was not able to obtain consistent data and limited my research to the interactions with the histone $\mathrm{H} 2 \mathrm{~A}-\mathrm{H} 2 \mathrm{~B}$ dimer at this point. 


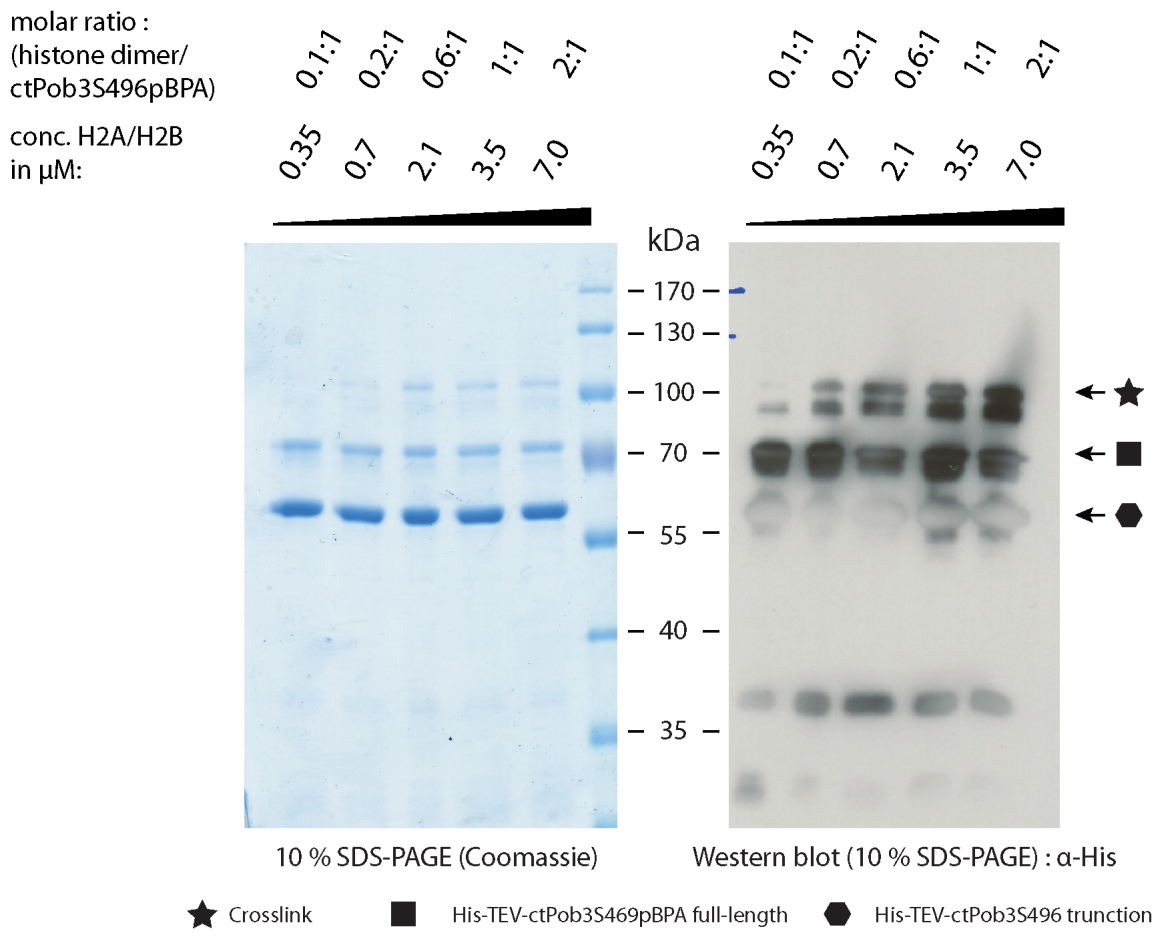

Figure 3.23: Crosslink experiments of the ctPob3S496pBPA mutant in presence of increasing histone H2A-H2B dimer amounts - Crosslink experiments were performed using Ni-NTApurified ctPob3S496pBPA protein. Increasing amounts of histone H2A-H2B dimer were added and crosslinked for 45 minutes on ice. Samples were boiled for 5 minutes at $95^{\circ} \mathrm{C}$ and analyzed by $10 \%$ SDS-PAGE (reaction per lane: $2.5 \mu \mathrm{g}$ ctPob3S496BPA mutant (total protein, NiNTA purified, final concentration: $3.7 \mu \mathrm{M}$ ); final buffer: $50 \mathrm{mM}$ TrisHCl $\mathrm{pH} 7.5$, $150 \mathrm{mM} \mathrm{NaCl}, 1 \mathrm{mM}$ EDTA, $1 \mathrm{mM}$ DTT, $75 \mathrm{mM}$ imidazole). The molar ratios are calculated using the total determined mass of the samples. Gel was prepared in duplicate whereas one was stained with InstantBlue ${ }^{\mathrm{TM}}$ and the other subjected to western blot analysis against the his-tag-epitope (PVDF Immobilon-P membrane, $\alpha$-mouse IgG-HRP secondary antibody). The HRP-detection was done with the Amersham ${ }^{\mathrm{TM}}$ ECL $^{\mathrm{TM}}$ Prime Western Blotting Detection Reagent (5 seconds exposure).

\subsubsection{Purification of recombinantly expressed yNhp6a from Escherichia coli and its impact on in vitro crosslinking studies of ctPob3pBPA mutants to reconstituted mononucleosomes}

Expression and purification of yNhp6a: Several studies have investigated the role of the single HMG-box protein Nhp6a and its isoform Nhp6b in order to determine histone chaperone recruitment in yeast ${ }^{185[188}$. It is suggested to bind non-specifically to DNA allowing the recruitment of several chromatin remodelers, such as FACT complex. Here, it is essential for the in vitro interaction of the FACT complex with nucleosomes. For my crosslinking studies of ctPob3 with reconstituted mononucleosomes, I wanted to examine the role and impact of yNhp6a. I expressed Nhp6a 
protein $\left(13.1 \mathrm{kDa}\right.$ ) from plasmid pCDFD His-TEV-yNhp6a in E.coli Rosetta $^{\mathrm{TM}}$ 2(DE3) cells and purified it by NiNTA affinity chromatography using the N-terminal His-tag (figure 3.24 A.).

The induction of the T7 expression system with IPTG results in a protein band of approximately $15 \mathrm{kDa}$ (see samples before induction (b.i.) and harvested cells (a.i.)). The protein is highly soluble, since the majority is found in the supernatant fraction after centrifugation of the lysate. The non-bound protein fraction (FT.) of the affinity chromatography indicates efficient binding to the column material. The eluted His-TEV-yNhp6a protein shows minor impurities of higher molecular weight. A subsequently performed size exclusion chromatography could not further improve the purity of the protein. I reached a final protein yield of $5.3 \mathrm{mg}$ per liter expression culture.

\section{Binding studies of yNhp6a to reconstituted Xenopus laevis mononucleosomes:} I tested the functionality of the purified yNhp6a protein in a titration assay with reconstituted Xenopus laevis mononucleosomes. Binding of yNhp6a to mononucleosomes results in a slower migration of mononucleosomes in an electrophoretic mobility shift assay (EMSA) ${ }^{188}$. I titrated increasing amounts of yNhp6a to a fixed concentration of 400 nM reconstituted Xenopus laevis mononucleosomes (187 bp with Widom '601' sequence; figure 3.24 (B.) left). I performed native PAGE analysis and stained the gel with the GelRed ${ }^{\mathrm{TM}}$ nucleic acid stain. The mononucleosomes (species N2) appeared as a double band on the $4 \%$ native PAGE gel and were not fully saturated with histones during the reconstitution (black hexagon). This is confirmed by a significant residual amount of free DNA after the reconstitution (see (B) right figure; samples N2; circle). However, the titration of yNhp6a to these mononucleosomes results in slower migration of the nucleosome/DNA-yNhp6a complex in all tested concentrations. Furthermore, a saturation is reached with a yNhpa concentration of approximately $100 \mu \mathrm{M}$ (black star). The clear shift of the nucleosomes which is already observable at a yNhp6a concentration of $8.4 \mu \mathrm{M}$ indicates the binding reaction. Hence, I was assured that the yNhp6a is functional active. Next, I performed a salt stability assay of two independent mononucleosome reconstitutions, species N1 and N2, assuring stability for subsequent crosslink analysis (figure 3.24(B.) right). The reconstitution N1 shows less free DNA as compared to the N2 reconstitution. Additionally, N1 nucleosomes migrate as a single band in contrast to the less saturated N2 sample. The N1 population shows higher molecular weight bands migrating in a spaced ladder which is explained by the analysis of the reconstitution sample lacking histone octamers (DNA). The DNA template which is prepared from a plasmid carrying multiple copies of the $187 \mathrm{bp}$ sequence was not fully digested and therefore showed 
A. Expression and purification of yNhp6a from Escherichia coli

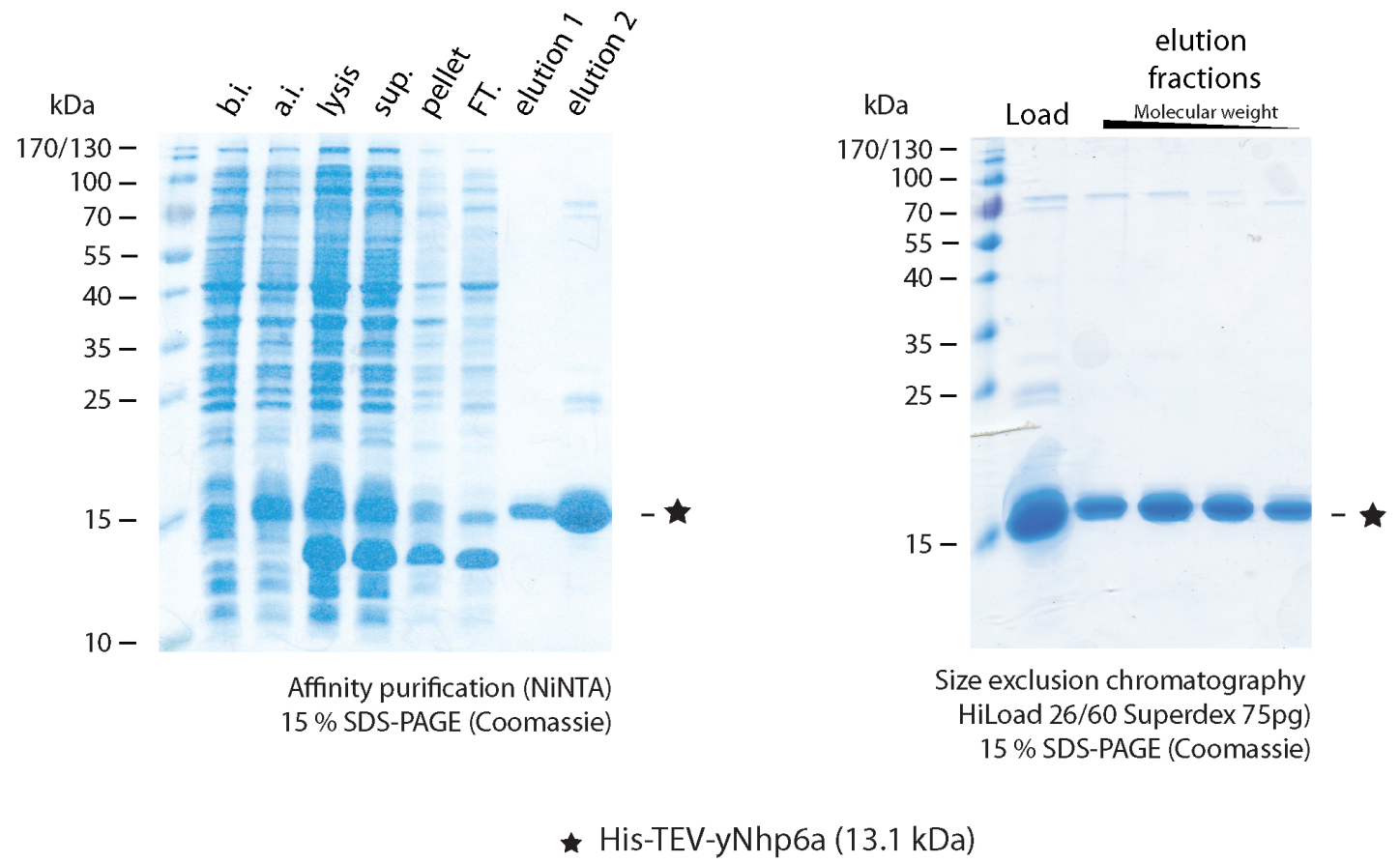

B. Binding assay of yNhp6a to reconstituted Xenopus laevis mononucleosomes

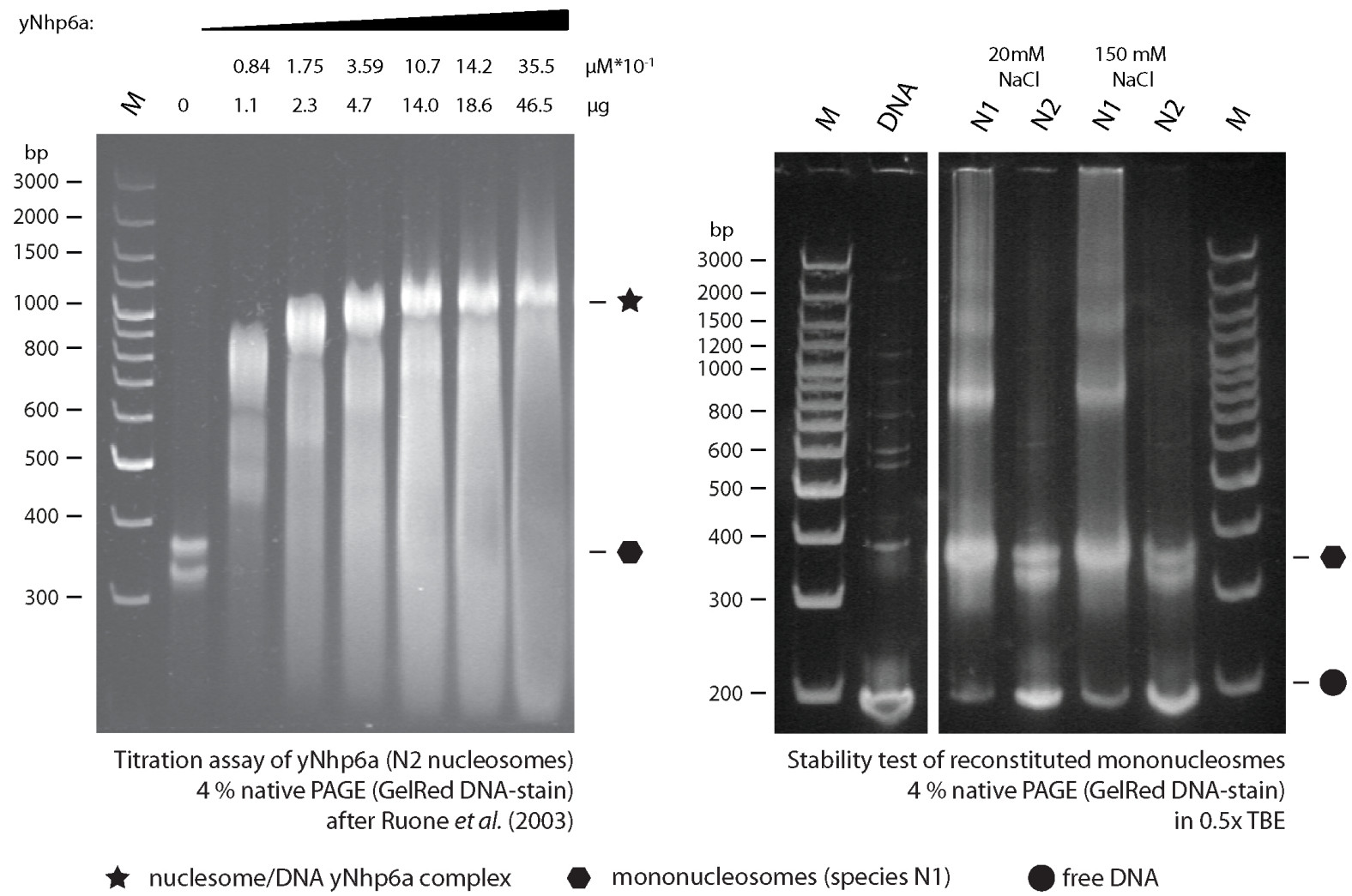

Figure 3.24: Purification of yNhp6a from Escherichia coli and binding studies to reconstituted Xenopus laevis mononucleosomes Caption next page. 
(Previous page.) A: 15\% SDS-PAGE analysis of yNhp6a expression and purification as described in 2.2.2.4. Samples were taken before induction (b.i.) and before harvesting (a.i) and analyzed by SDS-PAGE ( $150 \mathrm{mOD}_{600}$ per lane). Cells of $4 \mathrm{~L}$ expression culture were lysed and the soluble supernatant (sup.) was used for NiNTA affinity chromatography (left). Proteins were eluted and appropriate fractions subjected to size exclusion chromatography (right). (sup.: supernatant after centrifugation; pellet: pellet after centrifugation; FT.: flowthrough, non-bound proteins; Load: pooled and concentrated sample applied to SEC). B: EMSA of His-TEV-yNhp6a with reconstituted Xenopus laevis mononucleosomes (left) and salt-stability assay of mononucleosomes (right). Binding assay (left): $400 \mathrm{nM}$ mononucleosomes (reconstitution N2) were treated with increasing concentrations of yNhp6a $(8.4 \mu \mathrm{M}-355 \mu \mathrm{M})$ in $1 x$ BM buffer and incubated for 30 minutes at $30^{\circ} \mathrm{C}$. Samples were loaded on a $4 \%$ native PAGE after Ruone et al. (2003) 188 ( 1 hour 30 minutes at $150 \mathrm{~V}$ in $0.5 \mathrm{x}$ TBE). The gel was stained with the GelRed ${ }^{\mathrm{TM}}$ nucleic acid stain and analyzed with a standard UV transilluminator. (M: GeneRuler $^{\mathrm{TM}} 100 \mathrm{bp}$ Plus DNA ladder). A salt stability test (right) was performed by adjusting the two independent nucleosome reconstitutions ( $\mathrm{N} 1$ and $\mathrm{N} 2,20 \mathrm{mM} \mathrm{NaCl}$ ) to a final $\mathrm{NaCl}$ concentration of $150 \mathrm{mM}$ (10 mM TrisHCl pH 7.5, 150 mM NaCl, 1 mM EDTA, 1 mM DTT, 20\% (v/v) glycerol). Samples were analyzed by $4 \%$ native PAGE. The gel was analyzed as described above. (DNA: Reconstitution sample without addition of histone octamers as control.)

a similar ladder pattern. Therefore, reconstitutions with this template DNA might result in minor amounts of nucleosome arrays of higher molecular weight. Nevertheless, for subsequent crosslinking the N1 population was used. An increase of the $\mathrm{NaCl}$ concentration to $150 \mathrm{mM}$ did not effect the nucleosome to free DNA ratio. Hence, the nucleosomes are stable at a salt concentration which is used in a subsequent crosslink assay.

\section{In vivo crosslink assay of ctPob3S496pBPA with reconstituted Xenopus laevis} mononucleosomes in the presence of yNhp6a: Since ctPob3S496pBPA protein was shown to form crosslinks with the isolated histone $\mathrm{H} 2 \mathrm{~A}-\mathrm{H} 2 \mathrm{~B}$ dimer in a concentration dependent manner in vitro (see figure 3.23), I wanted to elucidate the crosslinking ability of ctPob3S496pBPA on reconstituted Xenopus laevis mononucleosomes. Therefore, I mixed ctPob3S496pBPA (m) or wild-type ctPob3 (w) with either mononucleosomes or histone $\mathrm{H} 2 \mathrm{~A}-\mathrm{H} 2 \mathrm{~B}$ dimers and subjected the samples to $365 \mathrm{~nm}$ light. Additionally, I performed an experiment with ctPob3S496pBPA and nucleosomes in the presence of the purified yNhp6a. Afterwards, samples were analyzed by SDS-PAGE and western blot against the his-tag-epitope detecting his-tagged ctPob3 proteins (figure 3.25).

Full-length his-tagged wild-type (w) and ctPob3S496pBPA (m) protein were detected in all samples (black square). Samples containing the pBPA mutant protein show the main truncation with a molecular weight of $55 \mathrm{kDa}$ (hexagon). As expected, crosslinking using the wild-type protein which does not contain the pBPA crosslinker showed no detectable crosslink adduct. Furthermore, pBPA mutant proteins showed no crosslink formation in the absence of dimers or nucleosomes (first 


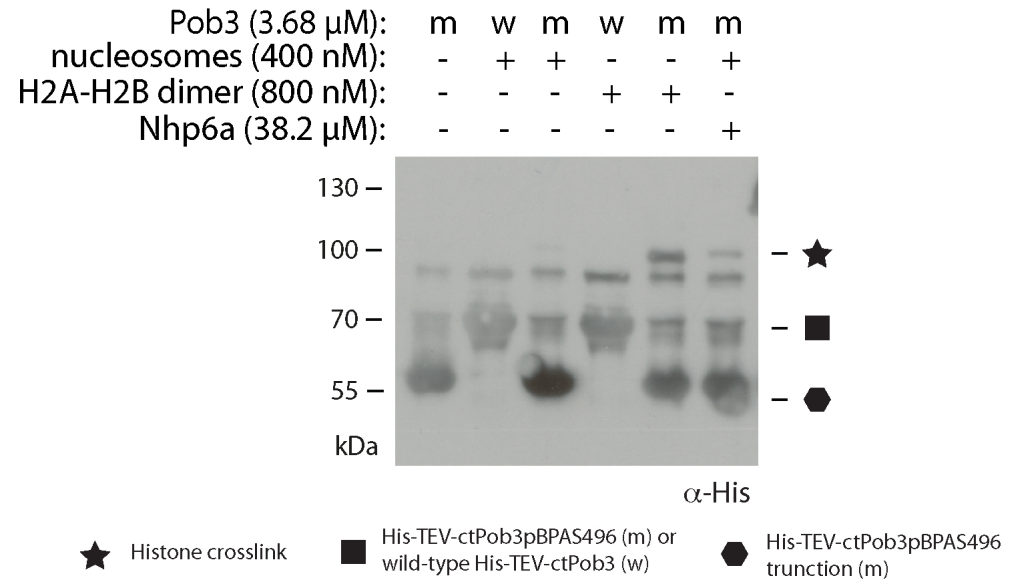

Figure 3.25: In vitro crosslink assay of ctPob3S496pBPA with reconstituted dimers and nucleosomes in the presence or absence of yNhp6a - Crosslink experiments were performed using purified ctPob3S496pBPA (m) and wild-type ctPob3 (w) proteins (final: $5 \mu \mathrm{g}$ total protein; $3.7 \mu \mathrm{M}$ ). Proteins were mixed with either reconstituted Xenopus laevis histone H2A-H2B ( $800 \mathrm{nM})$ dimer or mononucleosomes $(400 \mathrm{nM})$. Additionally, a sample containing ctPob3S496pBPA mutant proteins and nucleosomes was supplemented with $38.2 \mu \mathrm{M}$ purified Nhp6a. Samples were subjected to to $365 \mathrm{~nm}$ light for 30 minutes on ice (final buffer: $50 \mathrm{mM}$ TrisHCl pH7.5, $100 \mathrm{mM} \mathrm{NaCl}, 1 \mathrm{mM}$ EDTA, $1 \mathrm{mM}$ DTT, $75 \mathrm{mM}$ imidazole). Samples were boiled for 5 minutes at $95^{\circ} \mathrm{C}$ and analyzed by $10 \%$ SDS-PAGE and western blot against the his-tag-epitope (PVDF Immobilon-P membrane, $\alpha$-mouse IgG-HRP secondary antibody). The HRP-detection was done with the Amersham ${ }^{\mathrm{TM}}$ ECL $^{\mathrm{TM}}$ Prime Western Blotting Detection Reagent.

lane). However, experiments with nucleosomes show a faint crosslink product with a molecular weight of $100 \mathrm{kDa}$ (third lane, star) and the addition of yNhp6a (last lane) shows an increased intensity of that particular crosslink (last lane). The strongest crosslink signal is observed in a sample with free $\mathrm{H} 2 \mathrm{~A}-\mathrm{H} 2 \mathrm{~B}$ dimer. These experiments might indicate that ctPob3S496pBPA can also crosslink to reconstituted Xenopus laevis mononucleosomes. Nevertheless, the crosslink formation of that particular crosslink to mononucleosomes was substantially decreased in comparison to the free $\mathrm{H} 2 \mathrm{~A}-\mathrm{H} 2 \mathrm{~B}$ dimers, but it was increased by the addition of yNhp6a.

\subsection{Biological relevance of the acidic terminal tail of Pob3}

After analyzing the Pob3-CTD interactions to histones in vivo and in vitro, I wanted to elucidate their biological relevance. Interestingly, deletions of the CTD of either Spt16 or Pob3 are known to be lethal $82[180[182$. However, the reason is not fully understood. I started in-depth analysis of the CTD of Pob3 using bioinformatics, live cell imaging methods and competitive crosslinking studies. 


\subsubsection{Identification of a novel nuclear localization signal}

\subsubsection{Prediction of the C-terminal NLS using bioinformatics}

First I performed a protein alignment of the Pob3 protein sequences of several fungi, 12 ascomycetes and 1 basidiomycete. The alignment shows a high overall pairwise identity of $49 \%$ (mean pairwise identity over all pairs in column). My special focus was on the very acidic C-terminal residues (figure 3.26).

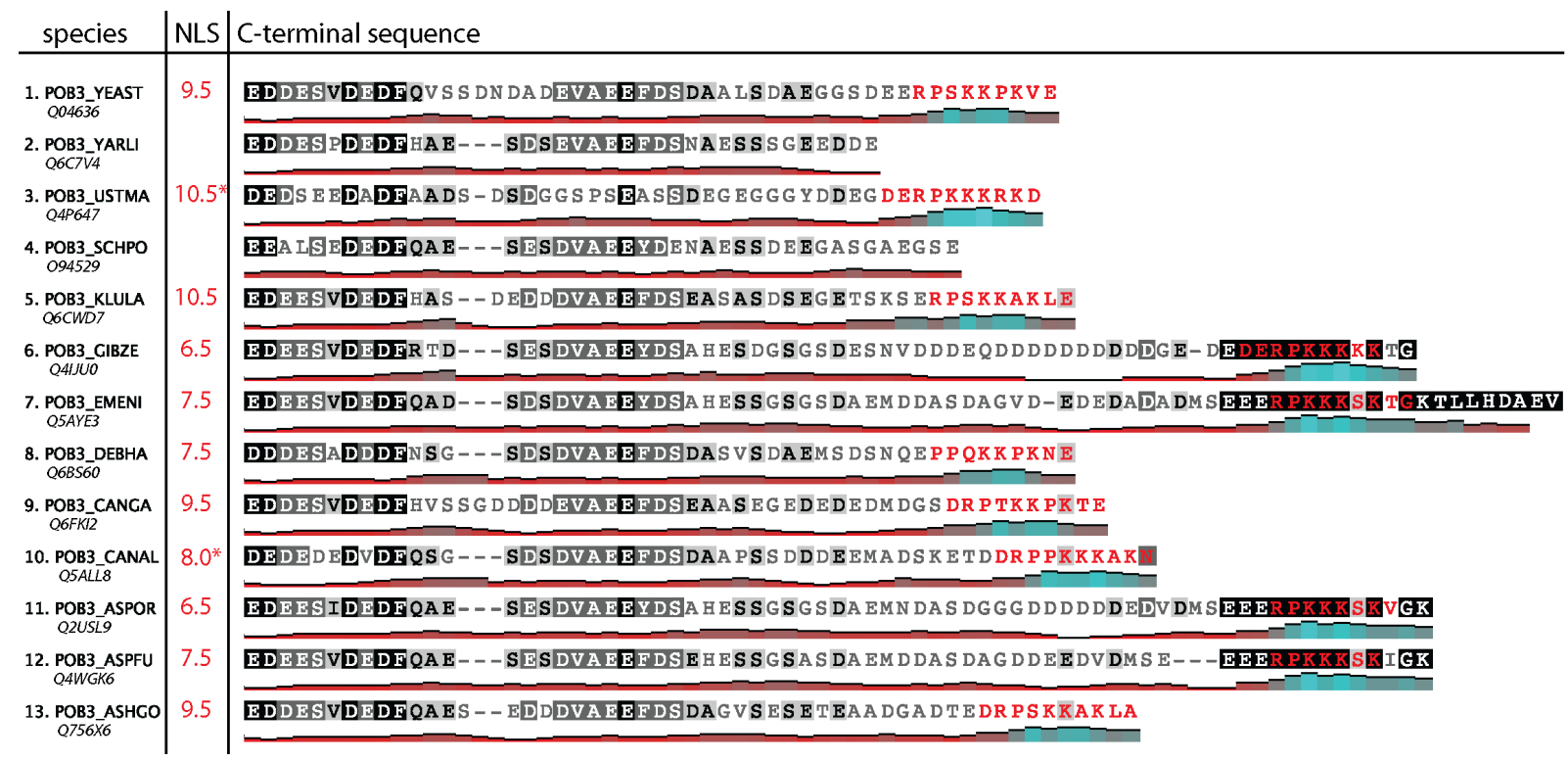

Figure 3.26: Segment of a protein alignment of 13 fungal Pob3 sequences showing the C-terminal region and their putative NLS sequence - Protein sequence alignment of Pob3 from 13 different fungal species. Accession numbers are shown in italics. Simple graphs below the sequence indicate the isoelectric point $(\mathrm{pI})$ in the surrounding environment (sliding window size: 5). Alignment parameters: matrix: Blosum65; gap penalty: 12; gap extension penalty: 3; global alignment with free end gaps; background similarity color code: identical (black, 100\% similar), dark grey (80-100\% similar, Blosum65 threshold: 1), light grey (60-80\% similar, Blosum65 threshold: 1), white (less than 60\% similar, Blosum65 threshold: 1). NLS analysis was done using cNLS mapper and putative signal sequence and NLS scores are shown in red ${ }^{298}$. For the NLS $\left(^{*}\right)$ analysis of Pob3 from Ustilago maydis and Candida albicans a glycine was added artificially to the C-terminus of the primary sequence.

Apart from Yarrowia lipolytica and Schizosaccharomyces pombe, the very C-terminal region contains a patch of basic amino acids additionally illustrated by the pI-chart (isoelectric point) below the primary sequence. Using the bioinformatic algorithm of cNLS Mapper, I could map nuclear localization signals to this basic patch in all tested sequences (amino acids in red) ${ }^{298}$. A score from higher than 8 indicates an exclusively localization to the nucleus by importin- $\alpha$ dependent nuclear import. Proteins with scores ranging from 7 to 8 partially localize to the nucleus. Lower scores show localization to both compartments $(3,4$, or 5$)$ or to the cytoplasm (1 or 2$)$. Similar results were obtained using the NLStradamus algorithm 299 . 


\subsubsection{Analysis of the putative Pob3 NLS by live cell imaging}

To verify this finding, I performed localization experiments using fluorescent microscopy. I cloned the Pob3 open reading frame from Saccharomyces cerevisiae as a N-terminal GFP fusion and expressed this plasmid-borne copy in wild-type yeast. Additionally, this plasmid was the basis for several deletion constructs which were performed by site-directed mutagenesis PCR (table 2.13 and figure 3.27A.). Living yeast cells were analyzed by fluorescence microscopy (B.).

Cells expressing the Pob3:GFP construct clearly showed a nuclear GFP signal (panel 3) in contrast to the expression of GFP-alone (panel 2) which was evenly distributed in the cytoplasm. Additionally, a strain carrying a genomically GFPtagged Pob3 confirmed the nuclear pattern of the plasmid-borne copy (compare panel 8 (grey) and panel 3). For the genomic control, the exposure needed to be decreased to achieve similar fluorescence intensities as were for the plasmid-borne Pob3-GFP fusion (from $500 \mathrm{~ms}$ to $100 \mathrm{~ms}$ ). However, nuclear localization is lost in plasmid mutants lacking the whole CTD (panel 4: $\triangle 458-552)$ as well as in a mutant lacking only the putative NLS (panel 5: $\Delta 544-552$ ). Interestingly, even a single point mutation (panel 6: K547M) lying in the importin- $\alpha$ classical NLS consensus sequence $(K-[K / R]-X-[K / R])^{300}$ shows delocalization of Pob3:GFP. However, the nuclear localization could be rescued by deleting the CTD leaving the very terminal residues (544-552) untouched (panel 7: $\Delta 458-543$ ). This confirms the C-terminal NLS and further shows the importance for proper localization of Pob3 in vivo.

\subsubsection{The effect of the Pob3 CTD-NLS on the histone $\mathrm{H} 2 \mathrm{~A}-\mathrm{H} 2 \mathrm{~B}$ dimer interaction}

The previously described histone dimer binding surface of the Pob3-CTD is situated very close to the basic NLS (figure 3.26). I was interested, whether interactions to the histone dimer and to a nuclear transport receptor are simultaneous or rather mutually exclusive. I took advantage of my in vitro crosslinking approach using the ctPob3S496pBPA mutant which showed crosslinking to histone H2A-H2B dimers (figure 3.23). The first step was to prove and establish an interaction between the ctPob3 protein and a nuclear transport receptor in vitro.

\subsubsection{Interaction studies between recombinantly expressed human importin- $\alpha$ and the Chaetomium thermophilum Pob3 homologue}

I compared the Pob3-CTD from Saccharomyces cerevisiae and Chaetomium thermophilum. A protein alignment shows a conserved long patch of 15 amino acids around the 
A.

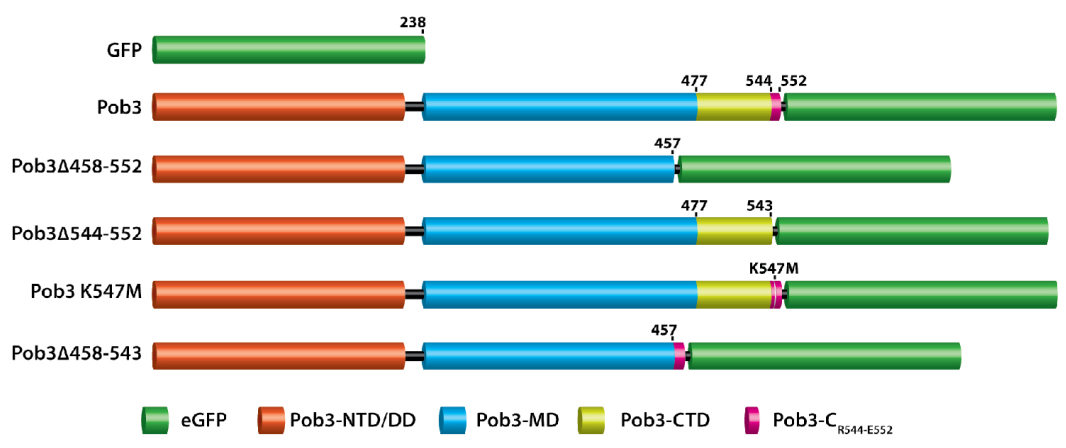

B.
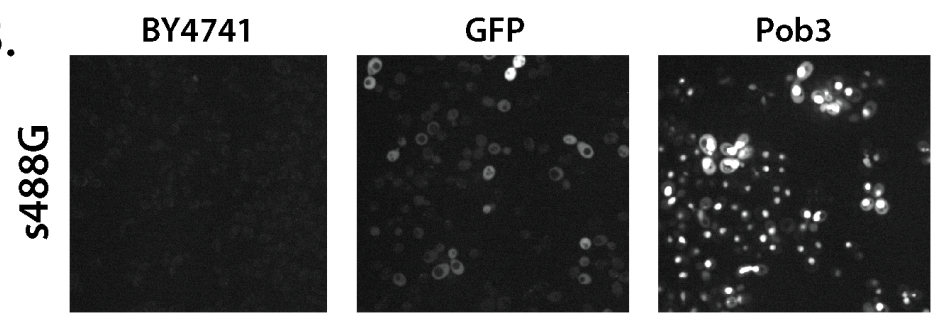

Pob3 $\Delta 458-552$
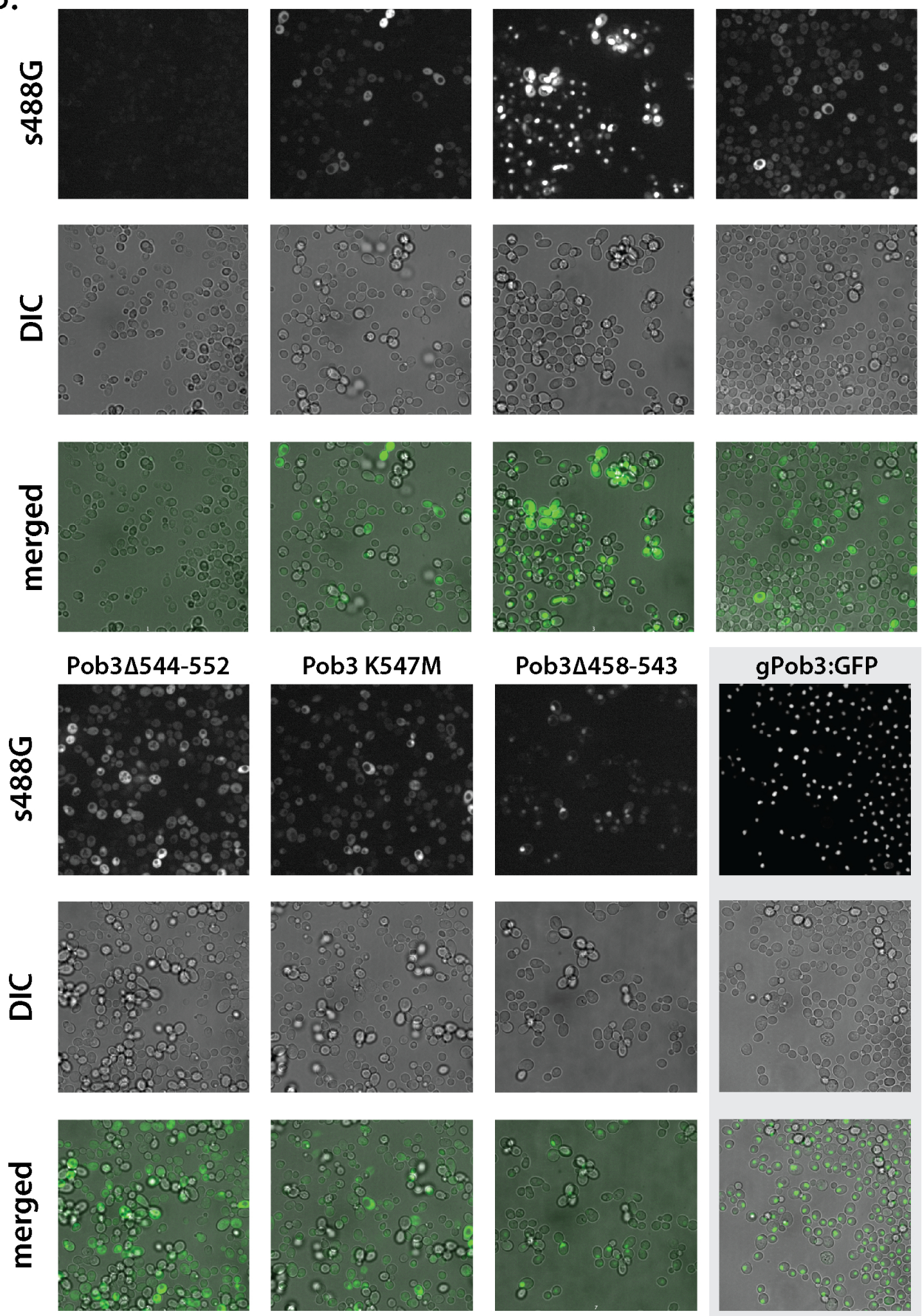

Figure 3.27: In vivo localization studies of Pob3:GFP constructs confirming the C-terminal NLS sequence in Saccharomyces cerevisiae Caption next page. 
(Previous page.) A: Schematic representation of different plasmid based Pob3 constructs used for localization studies. Numbering is based on the Saccharomyces cerevisiea sequence. The Pob3-ORF was cloned as a yEGFP N-terminal fusion (construct 2) using the pUG35 vector backbone. Several mutants were prepared by site-directed mutagenesis (constructs 3-6). B: Live cell imaging of BY4741 yeasts expressing different plasmid-borne Pob3:eGFP fusion proteins (panels 1-7). Samples were imaged under the same settings and exposures and processed using Fiji (512x512 pixel, s488G: 500 ms; DIC: $100 \mathrm{~ms}$; laser power: $100 \%$; gain: 1; intensification: 137). The last panel (grey 8, gPob3:GFP) shows a yeast strain carrying a genomically GFP-tagged Pob3 (s488G: 100 ms; DIC (Differential interference contrast): $100 \mathrm{~ms}$ ).

serine 507 for yeast and serine 503 for Chaetomium thermophilum respectively (figure 3.28 A.). The subsequent part of the Chaetomium CTD is longer as compared to the yeast CTD. Furthermore, the Chaetomium protein has a basic C-terminal patch too. Similar to yPob3, I identified a putative NLS with a score of 7.5 in this basic region (R563-K570). For interaction studies I additionally expressed and purified ctPob3 lacking the CTD, which contains the putative NLS, as a control (ctPob3 $\triangle$ CTD: His-TEV-ctPob3 $\Delta$ D467-G571). As nuclear transport receptor I chose the human importin- $\alpha$. The plasmid for expression in E. coli was a kind gift from Dr. Achim Dickmanns. It contains the importin- $\alpha$ open reading frame as a C-terminal fusion to a GST-tag. Additionally, the importin- $\alpha$ ORF is lacking the first 55 amino acids containing the auto-inhibitory importin $\beta$-binding domain $(\triangle \mathrm{IBB})$. Proteins, His-TEVctPob3, His-TEV-ctPob3 $\triangle$ CTD and GST-hImportin- $\alpha \Delta \mathrm{IBB}$ were purified as described in the material and methods part. The SDS-PAGE analysis of the protein expression showed specific binding of the proteins to the affinity matrix (figure 3.28 B.). This was indicated by a loss of protein signal at the expected molecular weight comparing the soluble supernatant (S) and the non-bound protein sample (FT). The elution (E) showed protein signals at these expected molecular weights. Therefore, each protein was successfully purified. In any case, samples were further subjected to size exclusion chromatography. Final yields were $0.8 \mathrm{mg}$ His-TEV-ctPob3 / L of expression culture, $2 \mathrm{mg}$ His-TEV-ctPob3 $\triangle \mathrm{CTD} / \mathrm{L}$ of expression culture and $4.8 \mathrm{mg}$ GST-hImportin- $\alpha \Delta \mathrm{IBB} / \mathrm{L}$ of expression culture. Each protein was of high purity already after the affinity chromatography.

For interaction studies I performed analytical size exclusion chromatography. Fixed amounts of single proteins or protein mixtures were loaded onto a S200 size exclusion chromatography column. The chromatograms were reported and collected fractions were analyzed by SDS-PAGE (figure 3.29).

The chromatography of each single protein resulted in one UV absorption maximum. Importin- $\alpha$ eluted at $11.4 \mathrm{~mL}$, ctPob3 FL at $11.6 \mathrm{~mL}$ and ctPob3 $\triangle \mathrm{CTD}$ at $13.2 \mathrm{~mL}$ respectively. The SDS-PAGE analysis of the elution fractions indicated the presence of each single protein. A molecular weight mass determination was done 
A.
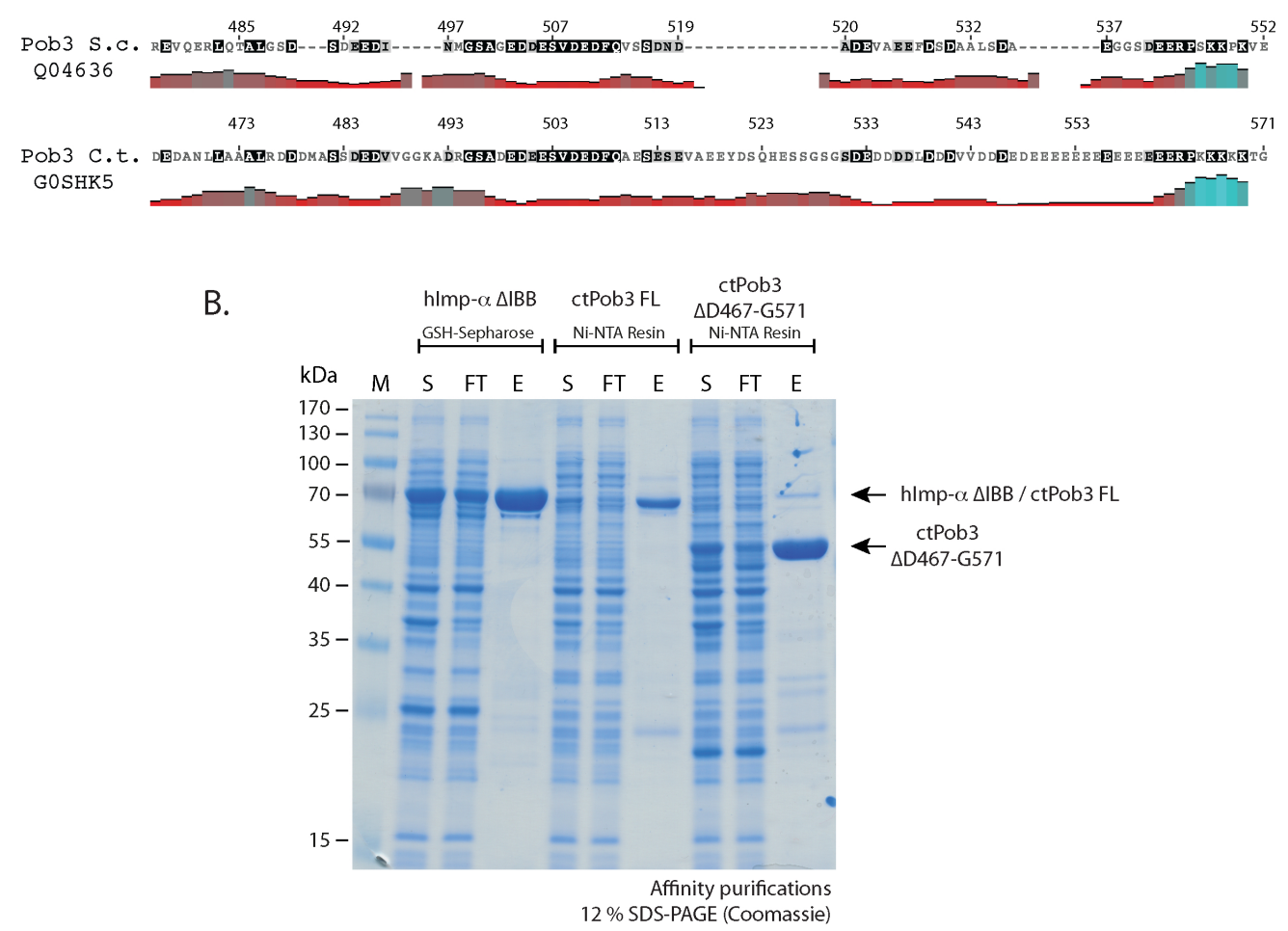

Figure 3.28: Saccharomyces cerevisiae and Chaetomium thermophilum Pob3-CTD protein alignment and SDS-PAGE analysis of recombinantly expressed importin- $\alpha$ and ctPob3 variants - A: Protein sequence alignment of Pob3-CTD from Saccharomyces cerevisiae and Chaetomium thermophilum. Accession numbers are shown below the names. Simple graphs underlying the sequence indicate the isoelectric point (pI) in the surrounding environment (sliding window size: 5). Alignment parameters: matrix: Blosum62; gap penalty: 12; gap extension penalty: 3; global alignment, Needleman-Wunsch algorithm; background similarity color code: identical (black, 100\% similar), dark grey (80-100\% similar, Blosum62 threshold: 1), light grey (60-80\% similar, Blosum62 threshold: 1), white (less than $60 \%$ similar, Blosum62 threshold: 1). B: 12\% SDS-PAGE analysis of affinity batch purification of three proteins: His-TEV-ctPob3 (67 kDa), His-TEV-ctPob3 $\triangle$ D467-G571 $(55.4 \mathrm{kDa})$ and GST-hImportin- $\alpha \Delta \mathrm{IBB}(78.8 \mathrm{kDa})$. (S: supernatant after centrifugation; FT: flowthrough, non-bound proteins; E: $5 \mu \mathrm{L}$ of elution fraction)

using a protein standard run. The logarithmic molecular weights of know size were plotted against their corresponding elution volumes and a linear regression was determined. Calculated molecular weights of the three sample proteins were compared with their experimentally determined molecular weights using the regression (figure 3.29 C.). In general, all proteins showed a higher molecular weight, experimentally determined from the size exclusion chromatography, than their actual calculated molecular weights. Importin- $\alpha$ was experimentally determined with $203 \mathrm{kDa}$ in contrast to the calculated molecular weight of $78.8 \mathrm{kDa}$. This difference can be explained by the presence of the GST-tag which is known to form dimers under physiological conditions. However, similar differences in molecular weight were observed for ctPob3 FL with $187 \mathrm{kDa}$ determined versus $67 \mathrm{kDa}$ calculated and ctPob3 $\triangle \mathrm{CTD}$ with 
A.

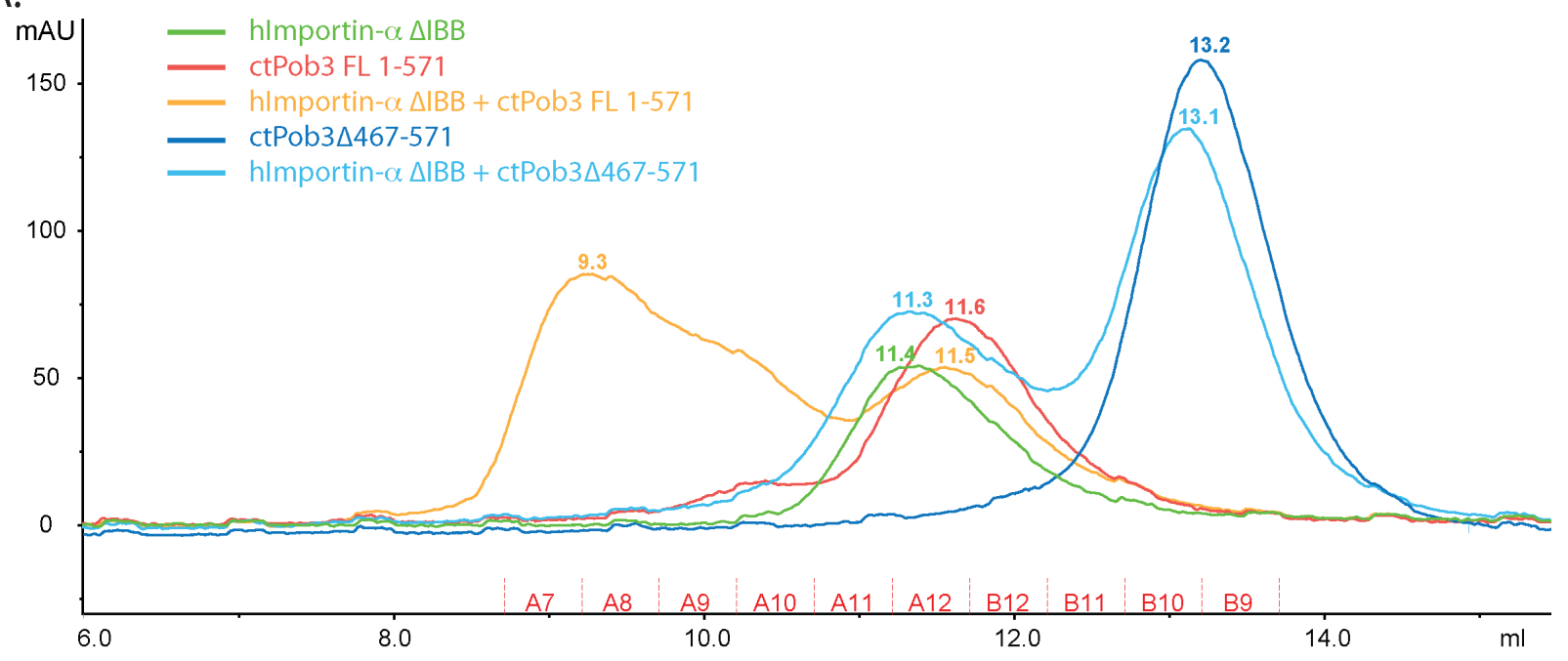

B.

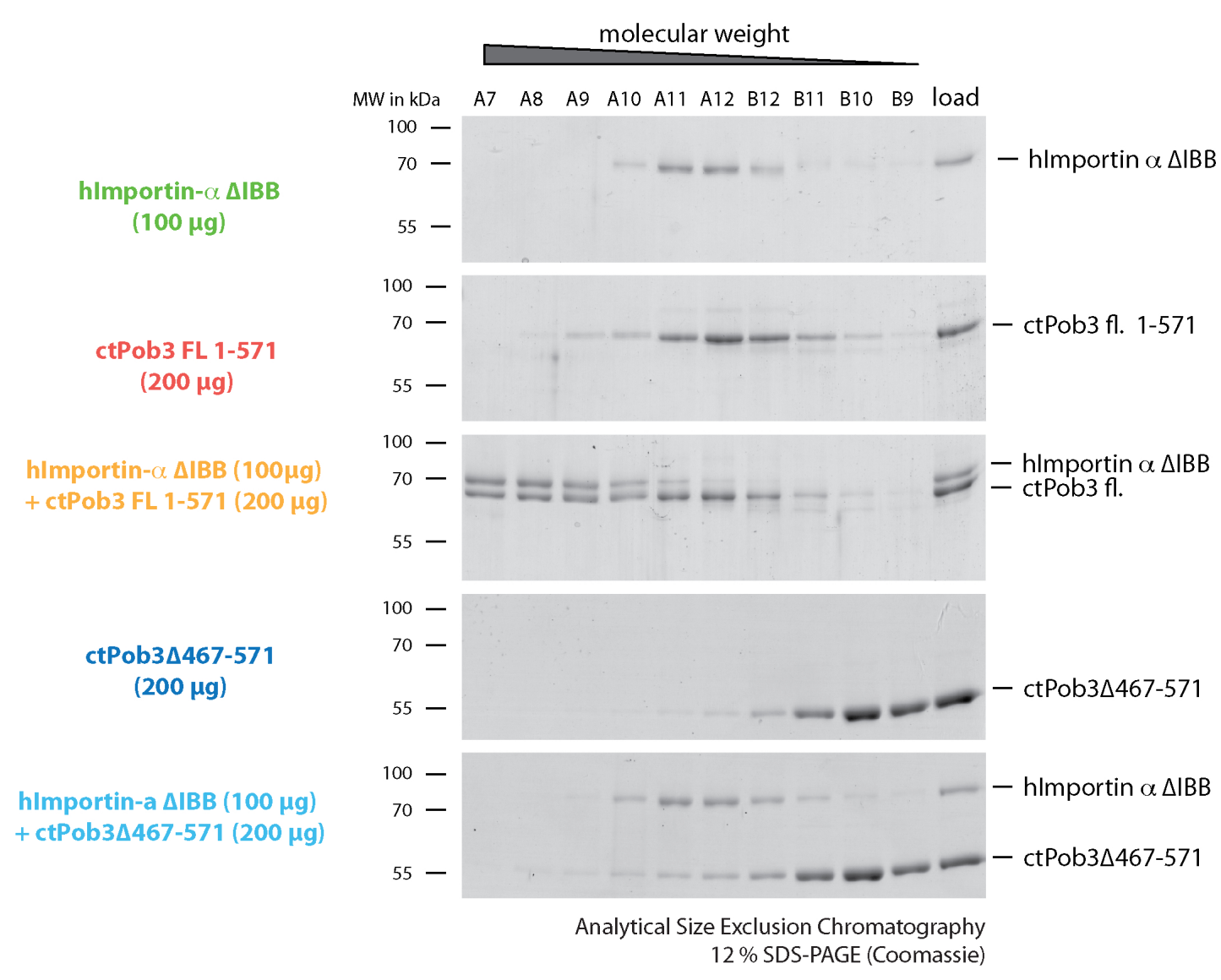

Figure 3.29: Analytical size exclusion chromatography of Chaetomium thermophilum Pob3 with recombinantly expressed and purified human importin- $\alpha$ - Caption next page. 
C.

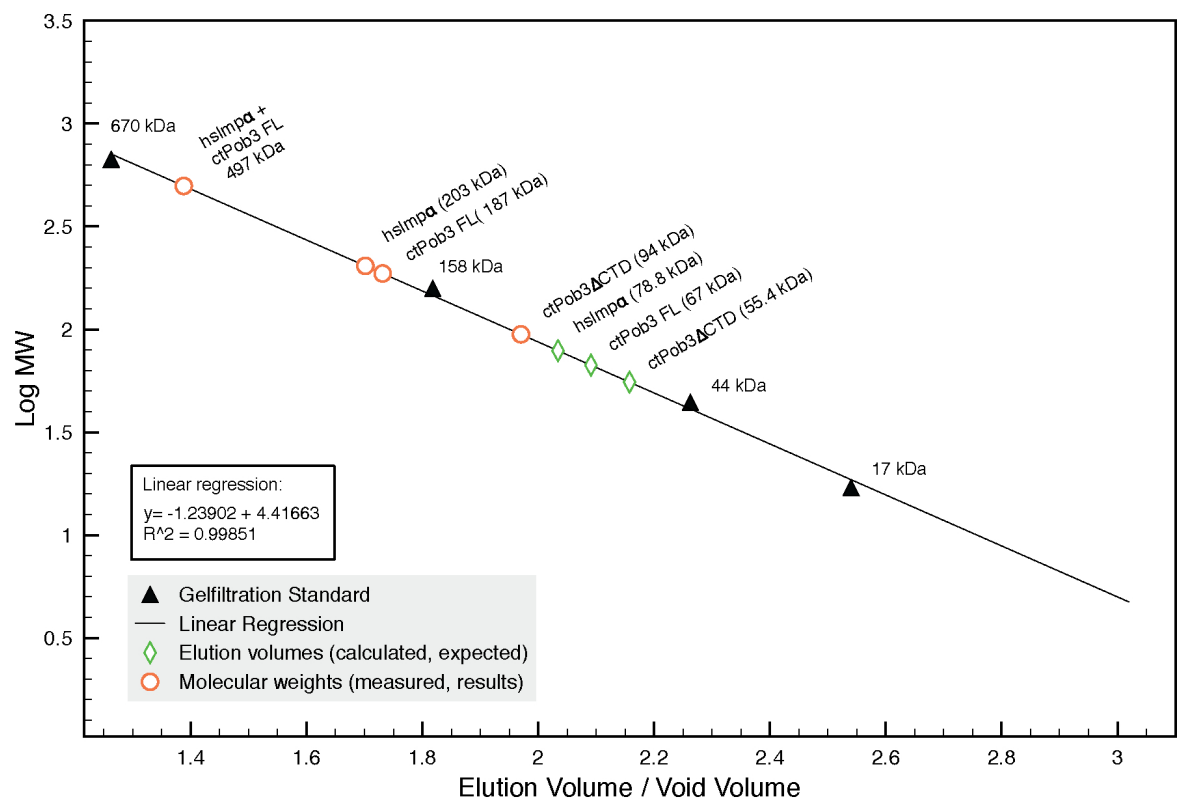

(Previous page.) A: Elution chromatograms of analytical size exclusion chromatography of samples containing ctPob3 variants $(200 \mu \mathrm{g})$, human importin- $\alpha(100 \mu \mathrm{g})$ and mixtures of both. Samples were applied onto a pre-equilibrated Superdex ${ }^{\mathrm{TM}} 20010 / 300 \mathrm{GL}$ column $\left(0.4 \mathrm{~mL} / \mathrm{min}\right.$ at $4^{\circ} \mathrm{C}$; Buffer: $50 \mathrm{mM}$ Hepes pH 7.2, $250 \mathrm{mM} \mathrm{NaCl}, 1 \mathrm{mM}$ DTT, 5 \% (v/v) glycerol). Collected fractions (0.5 mL) were analyzed by $12 \%$ SDS-PAGE (see part B). load: analytical gelfiltration loading sample; A7-B9: $8 \mu \mathrm{L}$ of each fraction were loaded. C: Molecular mass determination of the analytical gelfiltration samples using the Bio-Rad gel filtration standard. Logarithmic molecular weights of the standard proteins are plotted as a function of their corresponding elution volumes. Analytical gelfiltration samples (Pob3 and importin- $\alpha$ ) are plotted with their calculated elution volumes (green diamond). Experimentally determined molecular weights corresponding to part (A) are shown as red circles.

\section{$94 \mathrm{kDa}$ determined versus $55.4 \mathrm{kDa}$ calculated.}

For interaction studies between importin- $\alpha$ and Pob3, GST-hImportin- $\alpha \Delta$ IBB was mixed with either the full-length (FL) or the C-terminally truncated version of ctPob3 in a 1:2 total mass ratio and applied to a 200 size exclusion chromatography column. Strikingly, a sample containing importin- $\alpha$ and full-length ctPob3 shows two distinct absorption maxima at $9.3 \mathrm{~mL}$ and $11.5 \mathrm{~mL}$ in which the fractions from the first peak contained both proteins (importin- $\alpha$ and full-length ctPob3) in equal amounts (see SDS-PAGE: fractions A7-A10). This co-elution at a smaller elution volume compared to the individual proteins confirmed complex formation. The molecular mass of this complex was determined with approximately $500 \mathrm{kDa}$. In contrast, mixed importin- $\alpha$ with ctPob3 $\triangle$ CTD results in a chromatogram with two distinct absorption maxima at $11.3 \mathrm{~mL}$ and $13.1 \mathrm{~mL}$. These elution volumes correspond to similar volumes of their single runs with $11.4 \mathrm{~mL}$ and $13.2 \mathrm{~mL}$ respectively. The SDS-PAGE confirms their distinct segregated elution. Altogether, only full-length ctPob3 is able to form an importin- $\alpha$-ctPob3 complex whereas ctPob3 with a deleted CTD shows no interaction. 


\subsubsection{Competing crosslinking studies between histone and importin- $\alpha$ binding}

I confirmed the presence of a C-terminal NLS sequence on ctPob3 and reconstituted an importin- $\alpha$-ctPob3 complex. Since I observed also the interaction of the histone $\mathrm{H} 2 \mathrm{~A}-\mathrm{H} 2 \mathrm{~B}$ dimer in this region, I performed pBPA crosslinking assays using the ctPob3S496pBPA mutant and histone dimers either in the presence or absence of importin- $\alpha$. Samples were analyzed by western blot against the His-epitope of ctPob3 protein (figure 3.30).

For the crosslinking experiments with ctPob3 and histone dimer, I observed similar crosslinking behavior as shown already in section 3.3.2. Only in the presence of histone dimer and upon UV-treatment the higher molecular weight crosslink was detected (black hexagon). A sample with ctPob3 and importin- $\alpha$ was indistinguishable from its non-UV treated control. However, a reduction of the full-length ctPob3 signal (square) was observed in all samples containing importin- $\alpha$ which can be correlated to the presence of importin- $\alpha$ shown in the SDS-PAGE analysis. The reduction is a consequence of western blot transfer artifacts, since ctPob3 and importin- $\alpha$ share a similar molecular weight and importin- $\alpha$ is added in 2.1 molar excess. This massive protein amount might saturate the membrane at these spots which reduces the transferred and detected ctPob3 full-length protein. However, transfer of higher molecular weights, such as the crosslinked products with approximately $100 \mathrm{kDa}$, was not impaired since the background band at approximately $90 \mathrm{kDa}$ was not effected in samples containing importin- $\alpha$. The SDS-PAGE analysis confirmed these observations.

The addition of importin- $\alpha$ either before or after histone dimer addition resulted in a visibly reduced crosslink signal. This indicates that crosslink formation is impaired upon the presence of importin- $\alpha$ in the crosslinking reaction. Furthermore, preincubation with importin- $\alpha$ resulted in a slightly lower crosslink intensity compared to the experiment where the histone dimer was added first. In order to determine the extent of reduction caused by importin- $\alpha$, I repeated the experiment in triplicates pre-incubating ctPob3 with importin- $\alpha$ and subsequently adding histone dimer in a 2.8 molar excess (figure 3.30 B.). The quantification reveals a significant $37.5 \%$ decrease $(0.625$ fold) of crosslink formation upon the presence of importin- $\alpha$. 


\section{A. Competion crosslinking assay}

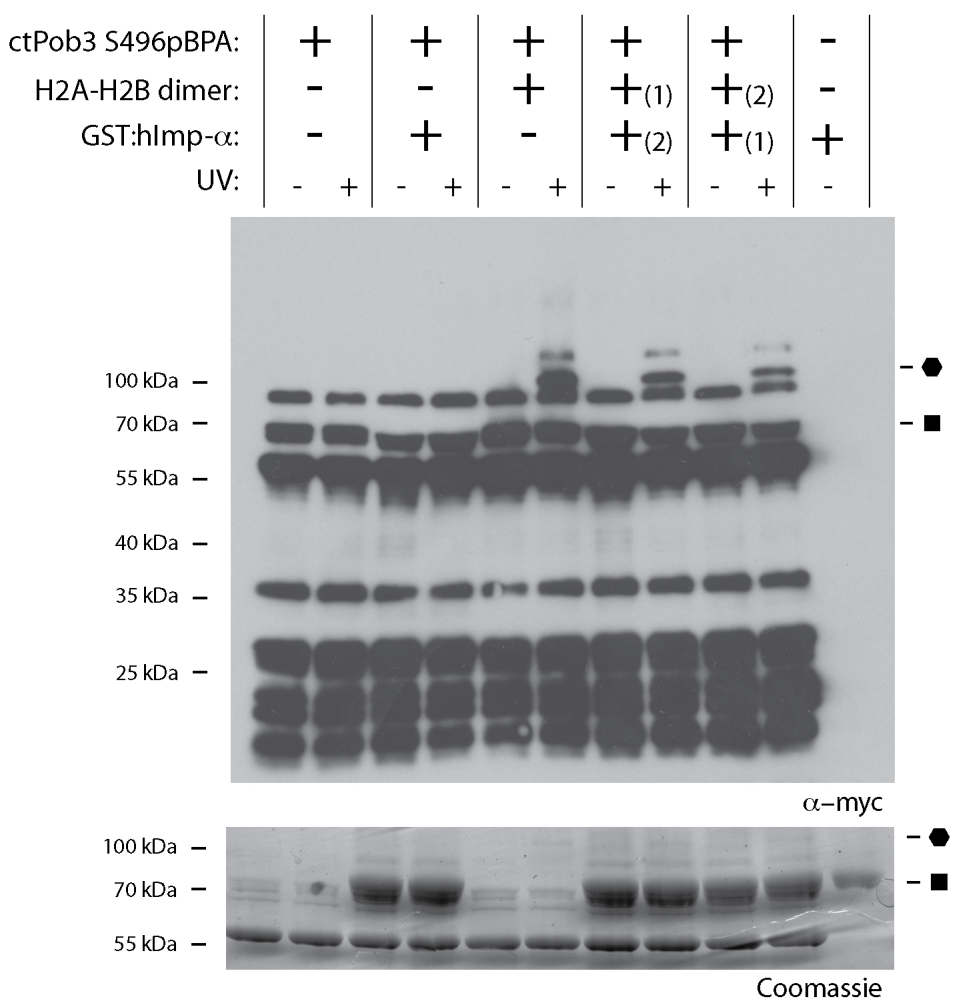

\section{B. Quantification (triplicates)}
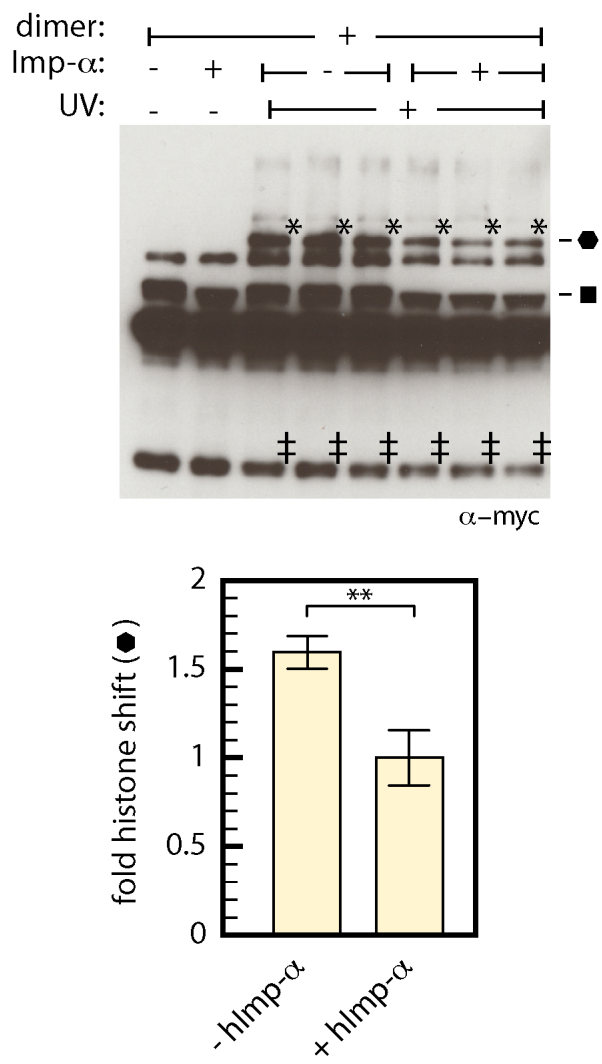

Figure 3.30: Competitive pBPA crosslinking assay of ctPob3S496pBPA with importin- $\alpha$ and histone H2A-H2B dimer - A: ctPob3S496pBPA was mixed with either GST-importin- $\alpha$, histone H2A-H2B dimer or both in a molar ratio of 1:2,1:1,6 (calculated upon total mass of NiNTA purified ctPob3). Samples were pre-incubated for 10 minutes before adding either importin- $\alpha$ or H2A-H2B dimer (numbers in brackets indicate the order). Samples were crosslinked for 30 minutes on ice (final buffer: $50 \mathrm{mM}$ TrisHCl pH7.2, $250 \mathrm{mM} \mathrm{NaCl}$, $1 \mathrm{mM}$ EDTA, $1 \mathrm{mM} \mathrm{DTT}, 80 \mathrm{mM}$ imidazole, $5 \%$ (w/v) glycerol). Samples were boiled for 5 minutes at $95^{\circ} \mathrm{C}$ and analyzed by $12 \%$ SDS-PAGE (amount per lane: $5 \mu \mathrm{g}$ ctPob3S496BPA mutant (total protein, NiNTA purified)). Gel was prepared in duplicate whereas one was stained with InstantBlue ${ }^{\mathrm{TM}}$ and the other subjected to western blot analysis against the His-tag-epitope (PVDF Immobilon-P membrane, $\alpha$-mouse IgG-HRP secondary antibody). The HRP-detection was performed with the Amersham ${ }^{\mathrm{TM}} \mathrm{ECL}^{\mathrm{TM}}$ Plus Western Blotting Detection Reagent (1 minute exposure). B: Quantification of a competition assay (top) using Fiji. Quantification was done in triplicates as described above. ctPob3S496pBPA was pre-incubated with importin- $\alpha$ for 10 minutes before adding the histone dimers. Molar ratio: 1:1,1:2,8 (ctPobS496pBPA:importin- $\alpha$ :histone-dimer). Amount per lane: $5 \mu \mathrm{g}$ ctPob3S496BPA mutant (total protein, NiNTA purified). Independent crosslink $\left(^{*}\right)$ to normalization signal $(\ddagger)$ ratios were calculated for each single triplicate. Average and standard deviation was calculated for samples with or without importin- $\alpha$. The average of samples containing importin- $\alpha$ samples was set to 1 . (Student's t-test, two-tailed, two-sample equal variance; $p=0.0046$; levels: ${ }^{*} \mathrm{p}<0.05,{ }^{* *} \mathrm{p}<0.01,{ }^{* * *} \mathrm{p}<0.001$ ) 


\subsubsection{Deletion of the acidic residues of Pob3-CTD in}

\section{Saccharomyces cerevisiae}

For further investigation of the Pob3-CTD in yeast, I wanted to generate genomic Pob3 mutants containing C-terminal deletions. Since, the previous results showed a novel NLS (R544-E552), which was shown to be crucial for the localization, I generated three internal deletions leaving the NLS untouched (table 3.1).

Table 3.1: Pob3-CTD deletion constructs for genomic integration - Pob3 region (amino acids P305E552 on pRS306 plasmid) was modified to produce several internal CTD deletions. Homologous recombination was used to integrate the mutated linearized plasmids into the yeast genome of MY721 yeast. The URA position (N-terminal or C-terminal) indicates the position of the URA marker in respect to Pob3 in 5'-3' direction (more details s. 2.2.1.12). A successful integration would result in a PCR product of indicated size using the primers \#CHR0068 and \#CHR0153 on isolated genomic yeast DNA.

Pob3 deletion Construct No. URA position PCR product size (bp)

\begin{tabular}{clll}
\hline \multirow{2}{*}{ none } & 1 & $\mathrm{~N}$ & \multirow{2}{*}{1222} \\
& 2 & $\mathrm{C}$ & \multirow{2}{*}{$\Delta$ Q458-E543 } \\
\multirow{2}{*}{$\Delta$ S491-E543 } & 3 & $\mathrm{~N}$ & \\
\hline \multirow{2}{*}{$\Delta$ A501-E543 } & 5 & $\mathrm{C}$ & \multirow{2}{*}{1065} \\
& 6 & $\mathrm{~N}$ & \multirow{2}{*}{1095} \\
\hline
\end{tabular}

For integration into the yeast genome, I took advantage of homologous recombination in yeast. In contrast to conventional methods, I transformed linearized plasmids instead of PCR products. The plasmids are carrying a region of Pob3 (P305-E552) with deletions or the wild-type sequence as indicated. Linearization was performed with MunI cutting the Pob3 fragment between the codons for Q329 and L330. This linearized plasmid can be utilized by yeasts homologous recombination machinery to be integrated into the genomic Pob3 ORF at this position. The plasmid backbone (pRS306) has the URA auxotrophy marker for later selection of successful integration (for details see section 2.2.1.12). First, I compared the amount of transformed colonies which were growing on medium lacking uracil. For deletions, $\Delta$ S491 and $\Delta$ A501, similar amounts of colonies compared to the wild-type integration were observed. In contrast, for the $\Delta \mathrm{Q} 458$ integration the amount was approximately a magnitude lower as compared to the wild-type integration. Differences concerning the direction of the Pob3 region on the plasmid (URA position), were not observed. I replated single colonies and isolated the genomic DNA from several clones. Successful inte- 
gration was tested by PCR analysis. The forward primer binds in the genomic region of Pob3 upstream of the Pob3 region which is present in the integration plasmids and the reverse primer binds to the most $\mathrm{C}$-terminal region of Pob3. Hence, the formation of a PCR product of reduced size for the deletion experiments could be observed only if the cassette was integrated into the genomic Pob3 ORF (table 3.1; figure 3.31).

The integration of the wild-type sequence (none) as control resulted in a single PCR band at the approximate size of the expected 1222 bp (black hexagon). A similar band was observed using isolated genomic DNA of the parent strain MY721. As expected, the control with water instead of the template DNA did not result in PCR product formation. Interestingly, the analysis of the $\Delta \mathrm{Q} 458-\mathrm{E} 543$ deletion strains resulted in two PCR products (red rectangles in A). An additional gelelectrophoresis confirmed the presence of these two products with the sizes of roughly $1200 \mathrm{bp}$ and $1000 \mathrm{bp}$ (hexagon and square). The upper band matches the size of the wild-type control whereas the lower band matches the calculated size for successful integration. This might indicate that in these strains two variants, precisely the wild-type and the deleted version, are present. These strains were further tested for growth on selective media plates to check for their mating type. The MY721 strain contains the HIS5 gene under the control of a MATa-specific promoter and the LEU2 gene under a MAT $\alpha$-specific promoter, thus, the mating type can be easily tested 282 . The strains with the two Pob3 variants grew in the absence of leucine but were dependent on histidine in the medium. Therefore, these strains were still haploid ( $\alpha$-mating type).

Next, I analyzed the $\Delta$ S491-E543 and $\Delta$ A501-E543 truncations (figure 3.31B.). The wild-type control shows a signal as expected with approximately $1200 \mathrm{bp}$ (hexagon). The truncations show only one PCR product with a lower size. This matches the calculated sizes of $1095 \mathrm{bp}$ and $1065 \mathrm{bp}$ which would indicate a successful integration and therefore genomic deletion of these acidic residues in the CTD (star). A second gelelectrophoresis resolved the size differences even between the $\Delta S 491-E 543$ and $\triangle$ A501-E543 deletion (figure 3.31B., bottom gel: red rectangle). Finally, strains with deletions $\Delta$ S491-E543 and $\Delta$ A501-E543 were confirmed by sequencing (blue boxes).

\subsubsection{Influence of the genomic Pob3-CTD deletions on yeast viability during genotoxic and replicative stress}

The Pob3-CTD strains were further tested for growth defects on plates containing hydroxyurea (HU) ${ }^{217}$ or methyl methanesulfonate (MMS) $\underline{301 / 302}$ analyzing their behavior towards genotoxic or replicative stress. In addition to the wild-type BY4741 strain, the temperature sensitive pob3-L78R strain was tested. Serial dilutions of freshly grown yeast cultures were spotted on plates using a stamp. Plates were 


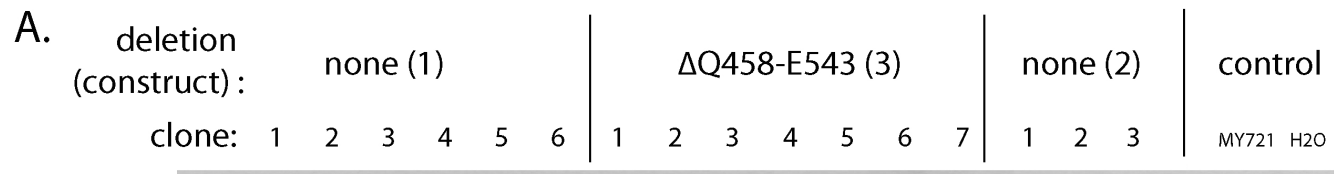
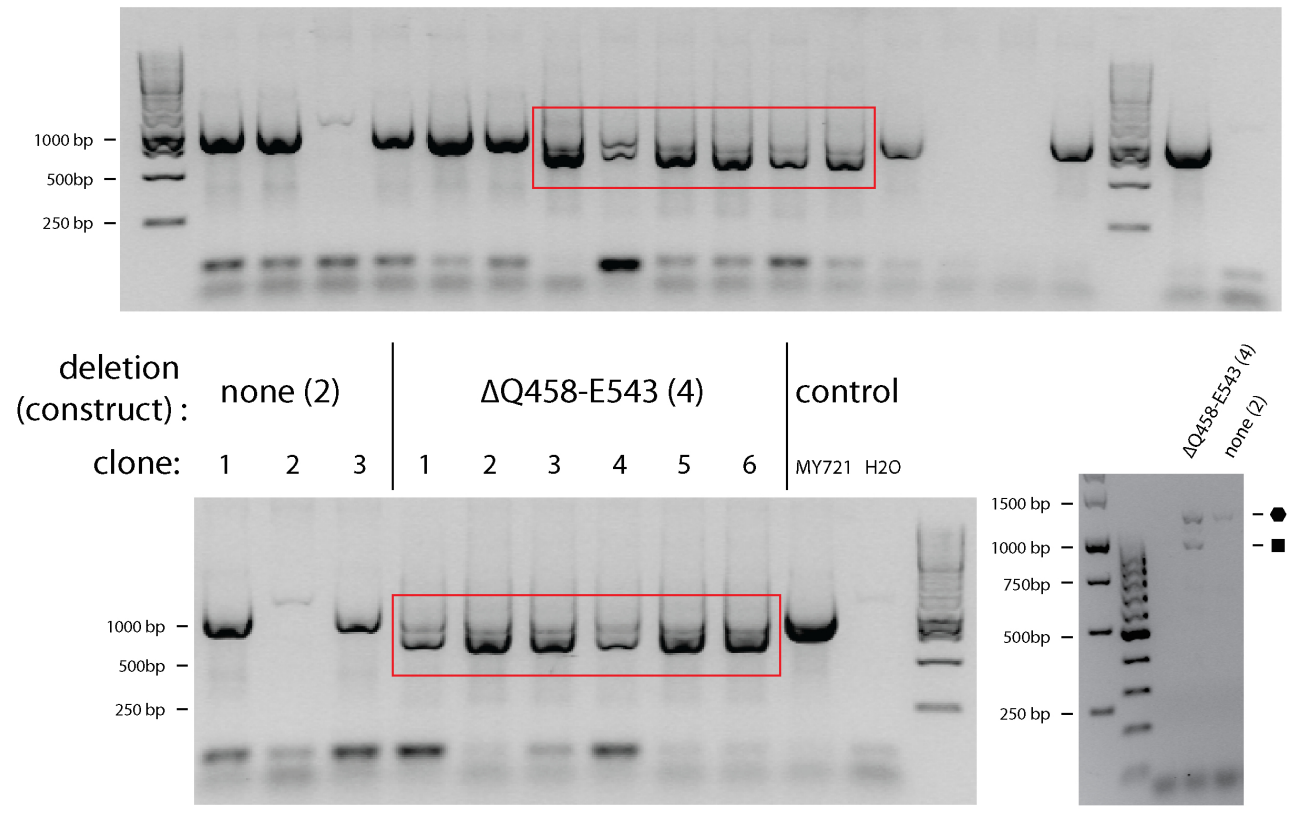

B.

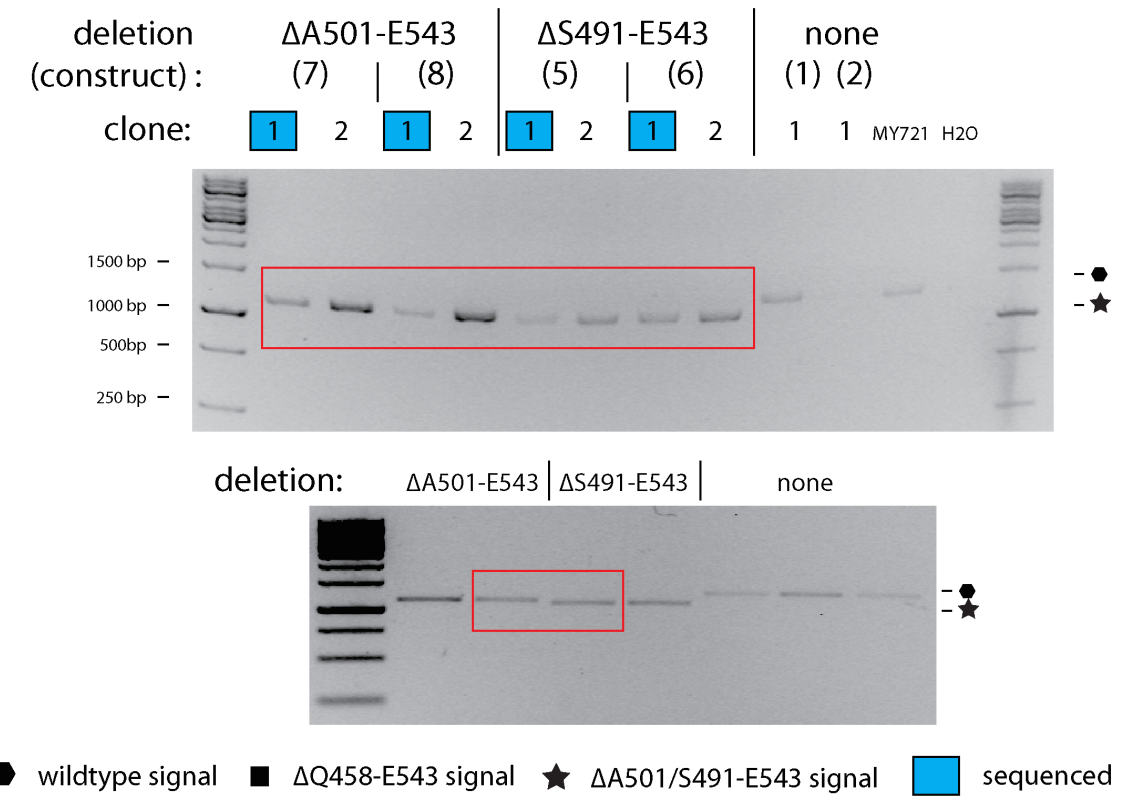

Figure 3.31: PCR-based strategy confirming the genomic integration of Pob3-CTD deletions - Different deletions in the CTD of Pob3 were genomically integrated transforming MunIlinearized plasmids into the MY721 yeast cells. Genomic DNA isolation of single clones was performed and successful integration was checked by PCR and agarose gel electrophoresis. Forward primer (\#CHR0068) anneals to the genomic region, not present in the integration construct whereas the reverse primer (\#CHR0153) anneals to the outermost C-terminal region of Pob3 (present in both). Expected products for successful integration are given in table 3.1. Analysis of the yeast strains with deletion constructs $\Delta$ Q458-E543 (A.), $\Delta$ S491-E543 and $\Delta$ A501-E543 (B.). The control integration construct (wild-type sequence) is indicated as "none". For a second control parental genomic DNA of MY721 was used. Numbers in brackets are indicating the URA position $(\mathrm{C} / \mathrm{N}$; for clarification see table 3.1 and figure 2.2). PCR products of selected strains were sequenced and the deleted region was confirmed (blue squares, sequencing primer: \#CHR0632). 
incubated at either $25^{\circ} \mathrm{C}$ or $32^{\circ} \mathrm{C}$ to detect potential temperature sensitive phenotypes of the $\Delta$ S491-E543 and $\triangle$ A501-E543 strains (figure 3.32).
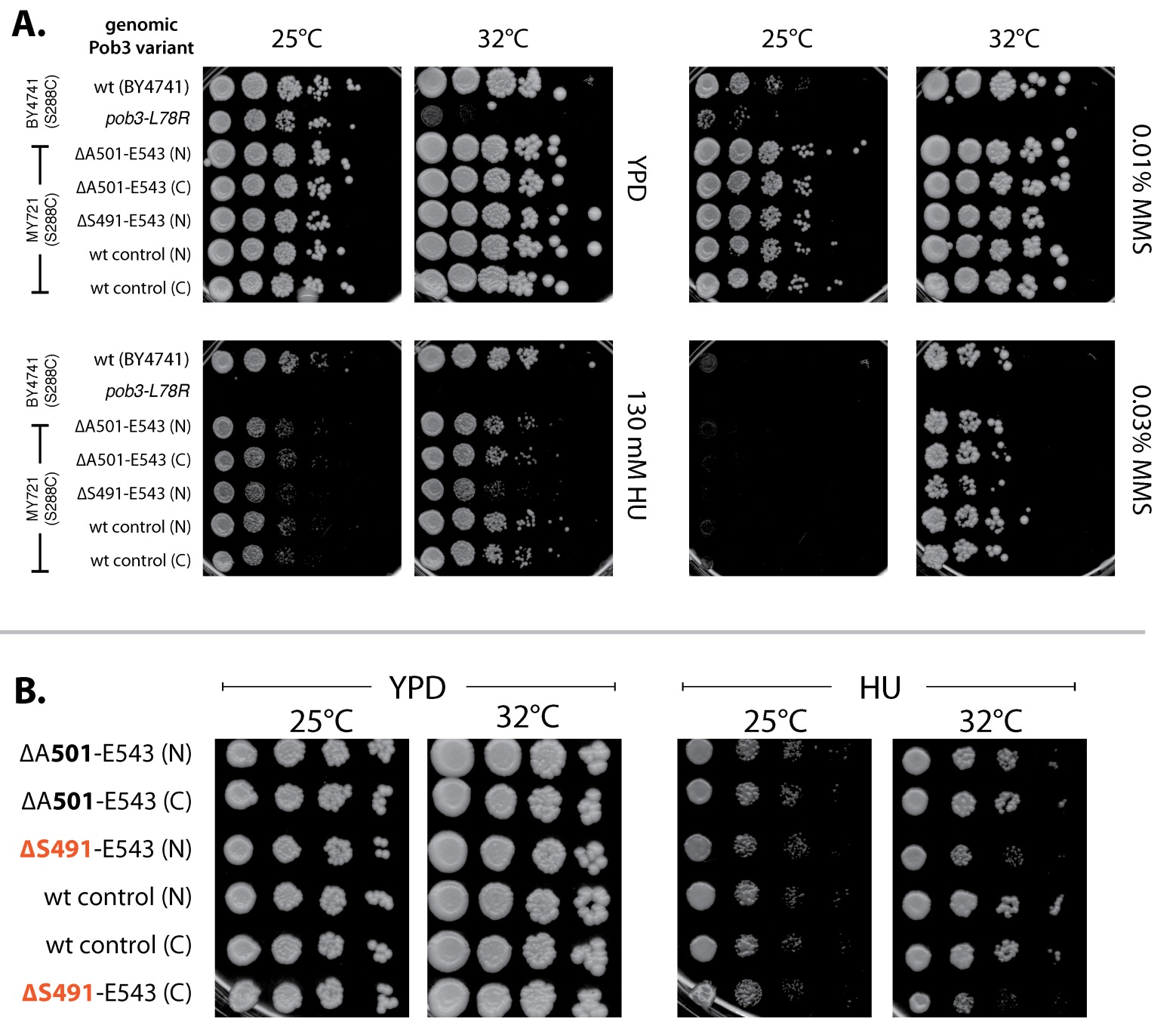

Figure 3.32: Spot assay of Pob3-CTD mutant yeast strains under genotoxic or replicative stress induced by hydroxyurea or methyl methanesulfonate - Cells of a fresh overnight culture were spotted as serial dilutions (1:10) starting with an $\mathrm{OD}_{600}$ of 1.85 on YPD media plates with $2 \%$ glucose in the presence or absence of $130 \mathrm{mM}$ hydroxyurea (HU) or $0.01 / 0.03 \%$ (v/v) methyl methanesulfonate (MMS). Plates were incubated either at $25^{\circ} \mathrm{C}$ or $32^{\circ} \mathrm{C}$ and analyzed 3 days after spreading. Strains represent $\mathrm{C}$-terminal deletion mutants which were confirmed by PCR and sequencing (figure 3.31. blue squares). The URA position is indicated in brackets $(\mathrm{C} / \mathrm{N}$; for explanation see table 3.1 and figure 2.2). Shown are similar experiments (A. and B.) whereas in B. the $\Delta$ S491-E543 (C) clone was added to the analysis.

All tested strain showed normal growth at $25^{\circ} \mathrm{C}$ on YPD plates. A slight growth defect is observed for the pob3-L78R strain. As previously shown and expected (figure 3.9), the pob3- $L 78 R$ showed a severe temperature sensitive growth defect on YPD upon incubation at $32^{\circ} \mathrm{C}$. Interestingly, none of the other strains including the $\triangle$ S491-E543 and $\triangle$ A501-E543 mutants showed any temperature sensitive effect on YPD at $32^{\circ} \mathrm{C}$. The wild-type BY4741 strain showed a minor growth delay on plates 
containing hydroxyurea whereas the pob3- $L 78 \mathrm{R}$ strain was inviable at $25^{\circ} \mathrm{C}$ and $32^{\circ} \mathrm{C}^{182}$. Comparing the BY4741 wild-type strain with the integration control strains in the presence of HU (last two rows), delayed growth of the integration control strains was observed at both temperatures. Furthermore, the $\Delta$ A501-E543 strain showed no observable difference to the integration control strains. Strikingly, the $\triangle \mathrm{S} 491-\mathrm{E} 543(\mathrm{~N})$ strain revealed a growth defect which is more prominent at $32^{\circ} \mathrm{C}$ than at $25^{\circ} \mathrm{C}$ in the presence of $130 \mathrm{mM} H U$. A similar experiment testing the $\Delta$ S491-E543 (C) strain (shown in figure 3.32 B., red) confirmed this defect. In comparison to the $\Delta$ A501-E543 which showed no observable phenotype to the control, the additional deletion of 10 more amino acids (S491-S500) of the $\Delta$ S491-E543 strain is sufficient to induce hydroxyurea sensitivity.

The DNA-damage reagent MMS was tested in two different concentrations. On plates containing $0.01 \%(\mathrm{v} / \mathrm{v})$, all strains were growing normal at $32^{\circ} \mathrm{C}$ with the exception of pob3-L78R which was inviable. However, at $25^{\circ} \mathrm{C}$ pob3- $L 78 R$ showed marginal growth. Furthermore, reduced growth at $25^{\circ} \mathrm{C}$ was observed for BY4741. In contrast, the integration controls and mutants grew normal. More severe defects were observed using $0.03 \%$ (v/v) MMS. Cells incubated at $32^{\circ} \mathrm{C}$ showed severe growth defects. The complete loss of growth of all strains was observed on plates with this concentration incubated at $25^{\circ} \mathrm{C}$.

In conclusion, the $\triangle$ S491-E543 deletion of the Pob3-CTD induces a mild hydroxyurea sensitivity which might indicate a potential role of the Pob3-CTD during replication. 



\section{Discussion}

My work used the technique of Genetic code expansion for the incorporation of unnatural amino acids in living yeast to study protein interactions of the histone chaperone complex FACT. A prerequisite for this particular technique is the expression of the protein of interest through an amber codon suppression that incorporates the unnatural amino acid resulting in full-length protein.

First I chose an appropriate expression system. The FACT complex subunits, Spt16 and Pob3, are essential in yeast ${ }^{158}$. The exchange of essential genes in yeast with mutated copies is feasible using the technique of "plasmid shuffling". This includes the endogenous knock-out of the gene of interest while rescuing the gene form a plasmid-borne copy. In a second step, a plasmid containing the mutated version can be added to the cells while selecting against the rescue-plasmid. In a study from 2008 Mohibullah and Hahn analyzed the interactions of the essential protein TBP (TATAbinding protein, Spt15) using the pBPA crosslinking system yeast ${ }^{273}$. They used a TBP shuffle strain and revealed that from 61 tested pBPA-containing positions in TBP only 24 gave viable cells although incorporation of tyrosine at all 61 positions led to viable cells. Thus concluding that the incorporation of pBPA is the main cause of inviability rather than substituting the position with a tyrosine. However, this might be also a secondary effect of the incorporation efficiency difference between the evolved pBPA-RS and the endogenous E.coli tyrosine-RS ${ }^{\sqrt[303]{3}}$ leading to decreased levels of TBP in the shuffle strain causing lethality of pBPA mutants. I think substitution of an essential wild-type copy with a lower expressed mutant copy, due to the generally lower incorporation efficiency as a consequence of the GCE system ${ }^{303}$, might not be beneficial and cause more cellular stress than an overexpression in vivo.

I decided to work with a system that expressed an additional copy of the FACT complex subunits, Spt16 or Pob3, containing pBPA instead of the system which limits Spt16/Pob3 protein levels from the beginning. Moreover, a loss of FACT activity is connected to increasing non-nucleosomal histone levels which can cause itself defects in transcription and DNA replication ${ }^{153304}$. Wittmeyer and Formosa showed that an overexpression of Spt16 (CDC68) does not influence yeast cell viability 158 . Nevertheless, a large yeast screen performing overexpression experiments from $2 \mu \mathrm{cron}$ plasmid with ORFs under the control of the strong galactose promoter revealed that 
overexpression of either Spt16 or Pob3 leads to a decreased vegetative growth rate $\mathrm{e}^{305}$.

These growth effects were similar to my observations. I expressed both proteins from a high copy plasmid ( $2 \mu \mathrm{cron}$ ) whereas Pob3 was under the control of the strong inducible galactose promoter and Spt16 under the control of its endogenous promoter. Induction of the full-length wild-type Pob3:9myc protein increased doubling time from initially 4-5 hours to 6-7 hours. In contrast, cells expressing Spt16 (even containing pBPA) had an average doubling time of $2.5-3 \mathrm{~h}$, thus, a moderate overexpression from the $2 \mu$ cron plasmid is preferable to the strong overexpression of the GAL promoter which additionally has to adapt for the change of carbon source from raffinose to galactose for induction.

I did not determine the absolute levels of plasmid-borne Spt16/Pob3 in comparison to the endogenous wild-type levels. However I did determine exemplarily the level of full-length Pob3:9myc expression in comparison to the Pob3:9mycS500pBPA level which can be estimated with roughly $10 \%$ of the Pob3:9myc expression level (figure 3.13 E.). This reduced level of amber suppression has two advantages: First, the full-length chaperone levels are lower reducing the slow growth effects and possible overexpression artifacts. Second, endogenous amber codons are suppressed in a similar range which is important for cellular survival. One reason for the reduced level is the competition between incorporation of the unnatural amino acid (pBPA) and termination of translation triggered by release factors $306 / 307$. Another cellular aspect which can alter the expression levels of amber-suppressed proteins in yeast is the nonsense-mediated decay (NMD) pathway 277/308. This eukaryotic pathway is responsible for degradation of mRNAs containing premature stop codons. For example, the efficient production of a GFP-TAG-mCherry construct using Genetic code expansion in worms was only achieved while the NMD pathway was reduced 309 . In yeast, a study by Wang and Wang ${ }^{310}$ revealed an increased unnatural amino acid incorporation using a NMD-deficient strain.

Generally speaking, the complexity of the Genetic code expansion including the expression of an orthogonal tRNA/tRNA-synthetase pair and the efficiency of incorporation during translation at the ribosome, makes it difficult to achieve equal expression levels of the amber-suppressed protein as compared to the wild-type copy. Nevertheless, using my expression strategy, neither major difficulties in ambersuppression during expression nor problems in full-length protein detection by western blot occurred throughout my work.

I successfully incorporated the unnatural crosslinker amino acids, pBPA and pAzF, into the histone chaperone FACT in Saccharomyces cerevisiae using Genetic code expansion. This is demonstrated exemplarily at the position S500 of Pob3 in figure 
3.14 (B.). I could clearly show that the original systems from Jason Chin et al. $\frac{255}{2}$ are specific for the incorporation of $\mathrm{pBPA}$ and $\mathrm{pAzF}$ since full-length protein was observed only in combination of the evolved tRNA/tRNA-synthetase pair with its cognate amino acid. However, I observe 10-20\% full-length protein even in the absence of the amino acid. This can be explained by a study from Chen et al. in 2007234. They used MRM (multiple-reaction monitoring) mass spectrometry to profile the incorporation specificity of several improved tRNA/tRNA-synthetase pairs in a quantitative manner. They could show that the system for incorporation of pBPA leads additionally to the incorporation of roughly $30 \%$ tryptophan and $7 \%$ leucine in response to an amber stop codon. The system for incorporation of $\mathrm{pAzF}$ was shown to be more specific roughly incorporating $\mathrm{pAzF}$ to $96 \%$ in response to an amber codon. Although they used an improved incorporation system, these results might explain the read-through of the amber codon which are matching my observations.

The system for incorporation of pBPA is generally feasible and consistent with recent literature $268|269| 270|271| 273$. However, for the incorporation of pAzF independent research groups achieved different results. The study from Nehring et al. ${ }^{[303}$, analyzed

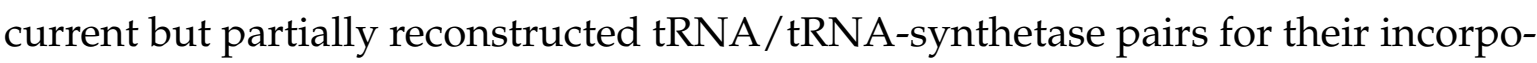
ration ability in vivo and tested the activation of unnatural amino acids by purified tRNA-synthetases in vitro. They were unable to reproduce the incorporation of $\mathrm{pAzF}$ into the pet-protein hSOD in yeast which was the proof of principle experiment of Jason Chin et al. ${ }^{255}$ in 2003 . In contrast to the work of Nehring ${ }^{303}$, a recent study from Berg et al. $\frac{311}{1}$ clearly confirmed amino acid dependent incorporation of $\mathrm{pAzF}$ into proteins in Saccharomyces cerevisiae. Additionally, they observed growth deficits in the presence of $\mathrm{pAzF}$ in the medium which was circumvented adding $\mathrm{pAzF}$ to high cell densities $\left(\mathrm{OD}_{600}=1\right)$ instead to very low cell densities. My results are consistent and confirming the later study. Using the original system from Chin $e$ t al. 255 , I successfully incorporated pAzF in response to the amber codon at serine 500 of Pob3 which was dependent on the presence of the amino acid. Additionally, I observed a similar inhibitory growth effect of the unnatural amino acid pAzF while present in the medium (section 3.2.1.3 and figure 3.14).

Before I started with an extensive crosslink screen of the FACT complex, I optimized several methodological parameters such as irradiation time or sample preparation and tested different yeast strains for crosslink analysis (section 3.1.3). I showed that the pBPA crosslink formation is dependent upon the irradiation time analyzing the Pob3S500pBPA crosslink (figure 3.3). A longer irradiation resulted in increased product yields. Moreover one crosslink could only be detected after 5 minutes. 
In my thesis the irradiation time varied usually from 10 to 30 minutes for in vivo experiments depending on the technical procedure. For pBPA and AzF crosslinking I used UV-light (UVA) with a wavelength of $365 \mathrm{~nm}$. This longer-wavelength light possesses less energy as compared to the UV-light with a wavelength of $254 \mathrm{~nm}$ which is generally used to induce DNA-damage repair due to formation of double strand breaks ${ }^{312}$. A guidance level is provided by a study from Das et al. ${ }^{137}$ claiming that UVB light with a wavelength of $312 \mathrm{~nm}$ is causing $1 \%$ of the the amount of DNA damage as compared to UVC light with $254 \mathrm{~nm}$. Therefore, the UVA light is even less harmful to the cells but can cause effects at very high energy dosages 313 . Nevertheless, the time of UV irradiation should be as short as possible but long enough to catch the crosslink products efficiently.

I tested a protease-deficient yeast strain (DSY5) lacking the two major vacuolar proteases (Pep4 and Prb1) to minimize protein degradation during cell lysis (figure 3.6). My results confirm the reduced degradation effect. However, I observed a slow-growth phenotype which can be explained by the DSY5 strain properties. DSY5 contains the integrated PGAL1-GAL4 expression cassette in the his3 locus. Therefore, addition of galactose promotes overexpression of the transcriptional activator Gal4 which in turn enhances overexpression of proteins under the GAL1 promoter. This might be useful for overexpression in yeast but not for my purpose since I want to generate moderate expression levels of Pob3pBPA mutants which are controlled in my system by the GAL promoter. This additional overexpression might explain the negative growth effects observed in the DSY5 strain. Hence, I decided to work with a the common yeast strain BY4741.

For the in-depth crosslink analysis of the FACT complex, I created a library of 214 individual amber mutants of the FACT. The design, creation, and characterization of final crosslink analysis was performed in a high-throughput manner. The time frame from primer design to the first readouts lasts about 2 month, thus, being optimized at several steps such as using 96-well blocks for library creation and 48-well blocks for yeast culture, crosslinking and extraction of total proteins. The library was used to incorporate pBPA at 186 different positions of the FACT complex including surface exposed as well as interior located positions. Observed crosslinks were positionand UV-irradiation-dependent (figure 3.7 and 3.8). This large screen allowed for the observation of commonalities and differences in the crosslink patterns which can not be revealed when testing single sites at a time. Aligning all the crosslinks revealed repetitive patterns which indicate interaction surfaces at adjacent sites (Spt16 R875D908; Y971-D996; Pob3 S500-E537). Despite the regional crosslinks, some crosslinks were abundant throughout almost the entire screen. These might indicate general 
interactions to huge cellular machineries involved in protein production, folding or degradation. The screen was performed using whole cell lysates, thus, no distinction between cytoplasmic and nuclear crosslinks can be made. The Pob3-screen shows a characteristic lateral crosslink pattern which starts at position E160. Each adjacent downstream crosslink position shows a similar crosslink but at a slightly higher molecular weight. This lateral pattern is consistent through the entire Pob3 screen and might indicate a crosslink of the full-length Pob3pBPA protein to the Pob3 truncation at this position which increases in molecular weight with every downstream amber position.

To test the integrity of the data, I compared the abundance of crosslink formation at specific sites with available structural data. As expected, positions buried in the inner core of the structure (e.g. Spt16-NTD (pdb 3BIQ): F117, F143, F432) are less prone to crosslink product formation than surface exposed residues (e.g. K108, K229, F250 and E426). Similar observations can be obtained analyzing the Pob3 middle domain (pdb: 2GCL). The hydrophobic residue F250 protrudes into the hydrophobic core of the $\beta$-barrel of the first PHD-domain and shows reduced crosslink formation whereas the adjacent residues G270, K271 and T272 are surface exposed on a small loop structure at the bottom of the $\beta$-barrel and show intensive crosslinks. Moreover crosslinking at position K271 reveals two higher molecular weight adducts which can not be found neither at G270 nor at T272. It is highly likely that an interaction which involves this loop structure should be present at all positions (G270-T272). However, the incorporation of the unnatural amino acid might perturb the interaction, thus, screening at selected positions is essential. All these selected examples indicating that surface structural data is corroborating the achieved crosslinking data and further reveal the potential of combined data analysis using crystal structures and in vivo crosslinking data.

Although similar levels of full-length protein were observed at the majority of sites, the Spt16 crosslink screen showed two regions with reduced Spt16:9myc protein level and crosslink formation (Spt16: Y810-D850 and T925-S965). The later region was recently shown to form a structurally important hydrophobic motif (Spt16 U-turn, amino acids 927-956) for histone H2A-H2B interaction by a crystallographic study of the Spt16 homologue from Chaetomium thermophilum 175 . The reduced full-length protein level might indicate a toxic effect of the expressed Spt16 protein or of the truncation at these positions respectively. Someone might speculate that especially the incorporation of the hydrophobic pBPA residue into the already hydrophobic U-turn motif produces this negative effect or simply folding and protein stability is perturbed due to the interference of pBPA residues at these sites. Together, the observed effects might indicate that this region indeed possess a role for functionality 
since the incorporation has a negative effect on the protein levels.

In order to draw further conclusions of the pBPA crosslinking scan, I functionally characterized every mutant using temperature sensitive Spt16/Pob3 yeast strains (figures 3.9, 3.10, 3.11 and tables 5.3, 5.4). Temperature-sensitive yeast strains are suitable for rescue experiments and genetic analysis. However, the molecular details and implications of the mutations is sometimes less understood. For the Spt16 pBPA dependent rescue assay, I used the spt16-ts/cdc68-ts strain carrying a G132D mutation which is found in many $c d c 68$-ts strains such as $c d c 68-1, c d c 68-11$ and $c d c 68$ 197180 . This mutation is structurally affecting the Spt16 N-terminal domain, most probably destabilising a $\beta$ strand alignment in the $\mathrm{N}$-terminal lobe of the domain (pdb: 3BIQ, see figure also 1.10). The mutation is connected to a drastic decrease of cyclin gene transcription upon restrictive temperature, thus, arresting the cell cycle at "START" (referring to $\mathrm{G}_{1}$-cell cycle phase in yeast) or early stages of S-Phase respectively $157|180| 212$.

I showed that plasmid borne Spt16:9myc was able to rescue growth at the restrictive temperature $\left(37^{\circ} \mathrm{C}\right)$ to a high level while the Spt16 amber mutants revealed different levels of pBPA dependent rescue behavior (figure 3.9, 3.10 and table 5.3). The different levels of rescue can have several explanations since several independent effects are overlapping in this read out. First, the rescue level is dependent upon the amount of pBPA protein produced, which can vary at different positions using the Genetic code expansion as described above. For example, the absence of fulllength protein at several sites in the Spt16-MD shown by western blot correlates with the loss of rescue ability for most positions (figure 3.7 and 3.10 positions: Y810D850 and T925-Y972). Second, I can not exclude an induced temperature sensitive phenotype resulting from the incorporated pBPA itself. This might produce additive effects at restrictive temperature. Third, the rescue by a partially produced protein, e.g. the produced truncation at the amber codon, might rescue the temperature sensitive phenotype independently of pBPA incorporation. The last fact might explain the pBPA-independent rescue behavior at both C-terminal domains of the FACT complex. Moreover, the imperfect orthogonality responsible for tryptophan and leucine incorporation in response to an amber stop codon 234 , might explain the rescue behavior even in the absence of pBPA at restrictive temperature.

Similar results were achieved testing the Pob3 amber mutant library (figure 3.9. 3.11 and table 5.4). The pob3-L78R strain (from the Boone library ${ }^{281}$ ) used in this thesis carried in addition to the L78R mutation a N187D, N331D and E503D mutation. As a known consequence, strains from yeast libraries must always be characterized before use. The original L78R mutation leads to a loss of Spt16-Pob3 heterodimers on 
the cellular level ${ }^{182}$ which might be explained by the position of the mutation in the Spt16-Pob3 dimerization domain. As for Spt16, expressed Pob3:9myc could rescue the temperature sensitive phenotype at restrictive temperature. However, in accordance with the previously observed effects of Pob3 overexpression in liquid culture, the growth these cells was already impaired at $25^{\circ} \mathrm{C}$. This confirms the preference to a moderate rather than strong overexpression. Nevertheless, the growth of most pBPA mutants at permissive temperature was restored probably due to the low incorporation efficiency of pBPA which reduces the full-length protein level while using Genetic code expansion.

In general, the interpretation of the rescue assay is difficult. However, the majority of the tested amber mutants showed an increased rescue behavior in the presence of pBPA at restrictive temperature. Therefore, I conclude that the majority of the pBPA mutant proteins are functional in vivo. Another possibility of testing the library would make use of the "plasmid shuffle" system in yeast as described above. However, my library amber plasmids contain the URA3 auxotrophy marker, thus, the classic shuffling method using 5-FOA (5-Fluoroorotic acid) ${ }^{314}$ for selection against the wild-type rescue plasmid (commonly URA3) can not be applied and alternative methods would be needed.

For the identification of the crosslinks, I performed shift assays as an easy and efficient technique for identification similarly as described in several studies $273|315| 316$. I focused on the identification of histone interactions of the FACT complex using genetically tagged yeast strains (figure 3.12). Using pBPA crosslinking, I could demonstrate for the first time that residues of the Pob3 C-terminal domain interact with the histones $\mathrm{H} 2 \mathrm{~A} / \mathrm{H} 2 \mathrm{~B}$ in a defined reproducible manner in vivo (figure 3.15). Furthermore, these interactions might indicate that the acidic tail is interacting with the H2A-H2B dimer over this surface in a stable conformation. Mechanistically, an interaction of the acidic domain of many histone chaperones with the basic histones is suggested $101 / 112: 175 \mid 181$. Just recently Dennehey et al. revealed the interaction between the acidic C-terminus of the chaperone Asf1 and histone $\mathrm{H} 3$ both in vitro and in vivo 315 . This matches nicely with my observed interactions of the Pob3 acidic Cterminal domain with $\mathrm{H} 2 \mathrm{~A} / \mathrm{H} 2 \mathrm{~B}$. However, I show interactions to $\mathrm{H} 2 \mathrm{~A} / \mathrm{H} 2 \mathrm{~B}$ at several positions whereas Dennehey et al. found only a single position in the CTD interacting with $\mathrm{H} 3$ using pBPA crosslinking approach. In addition to that, the acidic patch of Pob3 is highly conserved and even present in the SSRP1 in higher eukaryotes

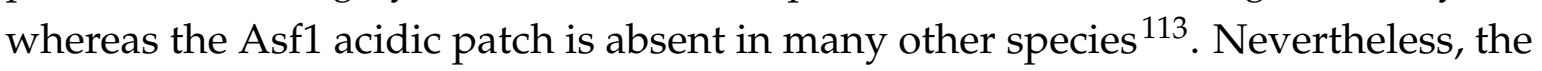
acidic C-terminal domains of both Pob3 and Asf1 are suggested to be structurally disordered, thus, it may be that structure is acquired upon binding to corresponding 
histones in vivo. Another study corroborating my results was published by Ramos et al. in 2010 317 . They performed Cryo-EM structure analysis of the histone chaperone Nucleoplasmin bound to H2A-H2B dimers. Nucleoplasmin contains three acidic tracts and the authors suggest based on their structural data that these are providing a flexible binding acidic surface and thereby contributing $\mathrm{H} 2 \mathrm{~A}-\mathrm{H} 2 \mathrm{~B}$ dimer interaction.

I performed several controls at the serine 500 of the CTD revealing that the specific $\mathrm{H} 2 \mathrm{~A}$ crosslink is predominantly found in the nucleus, independent of the H2A:fusion tag (3:myc or GFP) and absent in a genomically tagged H3 strain (figures 3.13, 3.12, 3.14). Therefore, concluding that a similar behavior can be expected for the residues tested in the scan of the Pob3-CTD which were reactive for $\mathrm{H} 2 \mathrm{~A} / \mathrm{H} 2 \mathrm{~B}$ (figure 3.15). Furthermore, I tested whether the interaction is an artifact of the crosslinker amino acid pBPA itself, thus, incorporating the crosslinker amino acid $\mathrm{pAzF}$ and performed a similar crosslinking experiment. In this respect, the Genetic code expansion technique proves its advantage as a versatile toolbox since for the incorporation of $\mathrm{pAzF}$ only the incorporation plasmid needed to be exchanged. I confirmed the interaction with $\mathrm{H} 2 \mathrm{~A}$ at serine 500 using pAzF instead of pBPA, thus, the interaction is not dependent on the crosslinker entity (figure 3.14). However the crosslink product formation of $\mathrm{pAzF}$ compared to $\mathrm{pBPA}$ at the same position under comparable conditions was significantly lower. This can be explained by several observations. Aryl azides, such as $\mathrm{pAzF}$, are activated in a range from $\sim 254$ to $400 \mathrm{~nm}$ depending on the ring substituents ${ }^{253}$, thus, activation with $365 \mathrm{~nm}$ might not be optimal. A general disadvantage of pAzF compared to pBPA is the irreversible nature of $\mathrm{pAzF}$ activation whereas $\mathrm{pAzF}$ further decomposes to a less reactive ketenimine after being in the active nitrene state for $100 \mu \mathrm{s}$ 253/258. The azide group can additionally be reduced to the non-reactive amine which might be problematic in biological systems 259 277. Moreover, a study from Nehring et al. $\underline{303}$ confirmed that the reduced form (AmF, p-amino-L-phenylalanine) is accepted by the evolved pAzF tRNA/tRNA-synthetase pair, thus, incorporating additionally the reduced form. A direct comparison of the $\mathrm{pBPA}$ and $\mathrm{pAzF}$ crosslinker by Lee and colleagues $^{266}$ revealed a 5 fold decrease in crosslink formation of a protein-DNA complex using pAzF instead of pBPA. All these examples confirm my experimental data. Nevertheless, I could confirm the interaction of the Pob3-CTD residue S500 with histone $\mathrm{H} 2 \mathrm{~A}$ using $\mathrm{pAzF}$ in vivo.

In my opinion, confirming interactions with different crosslinker amino acids, hence applying different crosslinker chemistries, is crucially important. Crosslinker amino acids can differ in their properties such as activation wavelength, crosslinking efficiency and chemical characteristics such as hydrophobicity and charge ${ }^{253}$. Therefore, a crosslinker at a certain position might influence or perturb an interactions 
or is not oriented properly to allow a covalent crosslink adduct. Such an example is beautifully demonstrated by the recent work of Lancia et al. from $2014^{318}$. They incorporated the crosslinker amino acids pBPA and pAzF into the Gal4 transcriptional activator and analyzed the well characterized interaction of F849 on Gal4 to the Gal80 repressor in vivo. Crosslinking with pBPA at this position was inhibited by two methionines in close proximity. By methionine to alanine mutations, the authors could restore pBPA crosslinking. This specific pBPA effect is known as "methionine magnet"256. Moreover, Lancia and coworkers showed that crosslinking with pAzF could be performed without mutating the methionine residues. This demonstrates beautifully the necessity of using different crosslinker amino acids due to different sequence context. Their conclusion that pBPA with its long lifetime ${ }^{254}$ and lower sol-

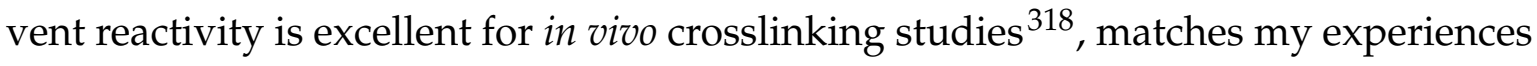
with this robust and reproducible crosslinker amino acid system.

The histone chaperone complex FACT is known to interact with histones $\mathrm{H} 2 \mathrm{~A} / \mathrm{H} 2 \mathrm{~B}$ as well as with $\mathrm{H} 3 / \mathrm{H} 4$ and several of these interactions are described by genetic interactions or direct biochemical analysis $82[174[175|176| 181 \mid 207$. Apart from the acidic Pob3-CTD crosslinking to histones $\mathrm{H} 2 \mathrm{~A} / \mathrm{H} 2 \mathrm{~B}$, at non of the other tested regions I could identify strong reproducible interaction to histones. Especially the acidic Cterminal domain of Spt16 which shows abundant crosslink patterns and is suggested to be involved in an electrostatic interaction to $\mathrm{H} 2 \mathrm{~A}-\mathrm{H} 2 \mathrm{~B}$ in vitro ${ }^{175}$, revealed neither interactions to $\mathrm{H} 2 \mathrm{~A} / \mathrm{H} 2 \mathrm{~B}$ in a comprehensive crosslinking scan (figure 3.16), nor to $\mathrm{H} 3$ tested at S986 in vivo. Only one putative crosslink to H2B at Y972 of Spt16 was observed but needs to be further investigated. I observed similar negative results at the N-terminal domain of Spt16 tested for $\mathrm{H} 3 / \mathrm{H} 4$ interactions ${ }^{174}$, at the surrounding of the recently described H2A/H2B hydrophobic U-turn motif of Spt16 tested for interactions with all four core histones ${ }^{175}$ and at the basic patch of the Pob3 middle domain tested for interactions with $\mathrm{H} 4{ }^{177}$ (figure 3.18, 3.19 and 3.17). A simple explanation might be that the positions used for the shift assays, which were chosen on structural, biochemical and crosslinking data, were not interacting with the histones per se or tested with the inappropriate histone partner. Additionally, the incorporation of the crosslinker might perturb the interaction at this position. Several other issues such as sequence context and crosslink nature and the possibility to test different crosslinker amino acids to solve this issue are discussed above. However, another probable explanation might be that the cellular context was not favorable for interactions to histones at tested positions or the interactions were too transient to be efficiently trapped. In vivo crosslinking studies can reveal controversial results concerning previously obtained in vitro data. This is demonstrated by the work of 
Berg et al. ${ }^{311}$ published in 2014. The authors incorporated pAzF at eight putative positions into Aha1, a co-chaperone for Hsp90 and performed in vivo crosslinking studies. Although native-coimmunoprecipitation confirmed binding of Aha1 with Hsp90 in vivo, non of the UV-dependent and site-specific pAzF crosslink products contained Hsp90. Furthermore, their mass spectrometry analysis revealed in vivo homodimerization of Aha1 at these crosslink positions. This study is supporting my observations concerning the absence of histone crosslinks at positions which are describe to be involved in histone interactions in literature. Moreover, it points out future directions of in vivo crosslinking in combination with proteomics such as mass spectrometry analysis for the identification of unknown and confirmation of identified crosslink interactions. Additionally, MS/MS approaches could not only to be used for identification of crosslink partners but even for the elucidation of the particular interaction site.

Therefore, I was trying to establish protocols for immunoprecipitation of crosslink products (section 3.2.3 and figure 3.20). I was able to enrich the Pob35500pBPA$\mathrm{H} 2 \mathrm{~A}$ crosslink using immunoprecipitation and western blot analysis. However, the SDS-PAGE revealed a high background signal in both samples. Furthermore, the technical conditions of the immunoprecipitation were not optimal. In any case, a successful enrichment of the crosslink by immunoprecipitation or similar enrichment techniques is additionally necessary since total protein extracts are usually not suitable for identification by antibodies directed against the interaction partner (personal observation). This is emphasized by several studies performing enrichment techniques and subsequent detection either with a directed antibody against the crosslink

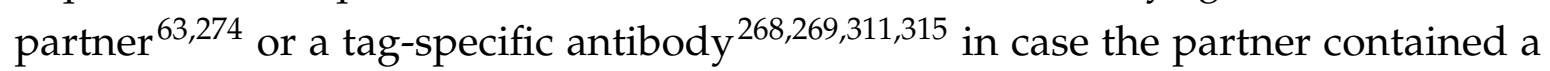
proteomic tag.

Mechanistically, the stable interactions of the Pob3-CTD to the histones H2A/H2B, putatively to the histone $\mathrm{H} 2 \mathrm{~A}-\mathrm{H} 2 \mathrm{~B}$ dimer, in a site-specific reproducible manner are matching current models of the FACT complex ${ }^{153 / 181}$. Although my current data does not allow to distinguish between the dimer eviction and global accessibility model, it definitely favors the current view of the FACT complex functioning by outcompeting DNA from histones to reduce non-nucleosomal histone-DNA interactions and therefore stabilizing the "open state"71|101|153|181]. My data suggests that the acidic C-terminal domain of yPob3 is involved in this process in vivo to a greater extent than previously suspected.

Since the acidic C-terminal domain showed these histone interactions in vivo, I intend to confirm them in vitro using the yPob3 homologue from Chaetomium thermophilum (section 3.3 and figure 3.21). The advantage of using proteins of this 
thermophilic filamentous fungus was demonstrated by Amlacher et al. in $2011^{319}$. The authors show that proteins from Chaetomium thermophilum possess improved properties in comparison to their mesophilic equivalents. Thee authors showed enhanced (thermo)stability, revealed increasing yields during heterologous expression in yeast and demonstrated that these proteins are therefore highly suitable for structural studies. It should be noted that the recently published structure of the Spt16 middle domain tethered to the histone H2A-H2B dimer was achieved using the ctSpt16 homologue $\mathrm{e}^{175}$.

I could successfully express and purify ctPob3 containing pBPA at several positions using Genetic code expansion in Escherichia coli ${ }^{233}$ (figure 3.21). I obtained reasonable yields with up to $9 \mathrm{mg}$ per liter expression culture. However, the amount of full-length ctPob3pBPA protein was estimated with $10 \%$ of the total protein amount since a huge fraction of the sample consist of a truncation due to translation termination at the amber codon instead of incorporating pBPA (figure 3.22 B.; hexagon). Nevertheless the amount of full-length ctPob3pBPA was still sufficient to perform in vitro crosslinking experiments, thus, no optimization of the incorporation system was necessary. I could show that several positions especially at the C-terminal domain of ctPob3 reveal shifts to a higher molecular weight in the presence of reconstituted H2A-H2B dimer and UV irradiation (S482, S496, Y520). This matches nicely with my in vivo crosslinking data of the Pob3-CTD. Furthermore, the two positions D280 and D310 show interactions to H2A/H2B indicated by crosslinks in the SDS-PAGE analysis and western blot (figure 3.21 and 3.22). However the equivalent positions in yeast (D293 and D323) were not tested in an in vivo shift assay for interactions to H2A/H2B. Unfortunately, I could not test the binding behavior of ctPob3 to H3-H4 tetramers since the crosslink mixture precipitated upon mixing of the single components.

In general, the observed in vitro crosslink products show different migration distances at different positions. This might be explained by the unusual structure of two crosslinked proteins migrating through the polyacrylamide gel. For further experiments, I selected the position S496 which is equivalent to the extensively examined S500 position in Sacharomyces cerevisiae. To exclude unspecific crosslink formation during my assays, I performed a titration experiment which clearly shows that the crosslink product formation is dependent on the presence of $\mathrm{H} 2 \mathrm{~A}-\mathrm{H} 2 \mathrm{~B}$ dimer (figure 3.23). This experiment further suggests that there is an excess of histone dimer over full-length ctPob3S496pBPA needed for the efficient formation of the crosslink. This is shown by the absence of the crosslink product at the lowest tested dimer concentration which represents a 1:1 ratio of full-length ctPob3S496pBPA to histone dimer (without the truncation). Unfortunately, it is technically difficult to resolve the crosslinks at the molecular weight of $100 \mathrm{kDa}$ and additionally separate the histones 
at the bottom of the gel at the same time. Western blot analysis with different antibodies directed against histone $\mathrm{H} 2 \mathrm{~A}$ or $\mathrm{H} 2 \mathrm{~B}$ would allow to draw conclusions on the histone species which interacted. Moreover, mass-spectrometry analysis of the crosslink products would allow me to elucidate the interactions in more detail.

In a recent study Dennehey et al. coexpressed Asf1 and $\mathrm{H} 3 / \mathrm{H} 4$ and performed crosslinking in Escherichia coli cell lysates to show interactions between Asf1 and $\mathrm{H}_{3}{ }^{315}$. Hence, using a less defined surrounding as the result of a bacterial overexpression system. On the other hand, I used purified proteins and reconstituted histone dimers and tetramers. Nevertheless, we both see the same effect in different migration distances of proteins upon crosslinkining to the same interaction partner.

I continued my analysis and performed crosslinking experiments of ctPob3S496pBPA with reconstituted mononucleosomes. It is shown that for a functional FACT complex the remodeling function of the HMG-box protein Nhp6 protein is needed $183 \mid 185[188$. Therefore, I recombinantly expressed and purified yNhp6a from Escherichia coli. The protein is highly soluble with a final yield of $5.3 \mathrm{mg}$ per liter expression culture (figure 3.24 A.). The expressed protein was functional since it performed binding to reconstituted mononucleosomes visualised by EMSA (B.). My EMSA assays are matching published results of Ruone et al. 188 and Formosa et al. 159 . The authors showed that Nhp6 binds free DNA as well as nucleosomes. In my experiments the reconstituted mononucleosome species (N2) contained a substantial amount of free DNA, thus in my EMSA assay with Nhp6a, I might observe combined effects of free DNA and nucleosome binding. Nevertheless, the mononucleosomes are efficiently shifted and therefore remodeled in the presence of an excessive amount of Nhp6a. Next, I tested whether ctPob3S496pBPA can crosslink to H2A/H2B in mononucleosomes compared to the crosslink ability to free $\mathrm{H} 2 \mathrm{~A} / \mathrm{H} 2 \mathrm{~B}$ as discussed above (figure 3.25). The ctPob3S496pBPA crosslink to H2A/H2B is strongly reduced using mononucleosomes as substrate. However, in the presence of Nhp6a the crosslink is strongly increased. This indicates that the Nhp6a-remodeled nucleosome is a better substrate for the ctPob3-CTD interaction than the closed canonical nucleosome. Thus confirming the importance of Nhp6a for the interaction with Pob3 as demonstrated for the Sp16/Pob3 heterodimer in the literature ${ }^{159 \mid 188}$. Interestingly, this crosslink ability is observed in the absence of Spt16.

In the last part of my thesis, I investigated the Pob3-CTD in respect to its biological relevance. Several histone chaperones and associated factors contain nuclear localization signals and/or export signals, e.g. members of the nucleoplasmin/nucleophosmin (NPM) and NAP-domain family (for a schematic overview ${ }^{112}$ ). My study contributed to this field of research, since I elucidated a nuclear localization 
signal at the terminal amino acids of the Pob3-CTD (amino acids 544-552) which is not described so far. It belongs to the family of the classical monopartite importin- $\alpha$ NLS with the consensus sequence $(K-[K / R]-X-[K / R])^{300}$. I confirmed the presence of the NLS using bioinformatics (figure 3.26), performing fluorescence microscopy localization studies in yeast (figure 3.27) and performing interaction studies between importin- $\alpha$ and ctPob3 in vitro (figure 3.29).

My microscopy studies clearly show that the disruption of the C-terminal NLS of the plasmid-borne GFP-Pob3 fusion construct results in mislocalization of this construct to the cytoplasm. The presence of the NLS might explain why some mutations in the yPob3-CTD are lethal which are described by Schlesinger and Formosa ${ }^{182}$. Moreover, Hondele et al. ${ }^{175}$ showed that the Spt16-CTD usually contains a similar monopartite importin- $\alpha$ NLS conserved throughout evolution apart from Saccharomyces cerevisiae. Thus, in yeast Spt16 localization might be dependent on the C-terminal NLS of Pob3 which would suggest that the FACT complex is imported to the nucleus as a fully assembled unit. To prove this hypothesis and gain a deeper insights into the nuclear localization of FACT, additional in vivo localization studies with fluorescently labeled Spt16 and Pob3 need to be performed. Especially extended data analysis using bioinformatics will contribute to solve this puzzle. The comparison of the species Schizosaccharomyces pombe and Saccharomyces cerevisiae can be used as an example. As discussed above, in baker's yeast the Pob3-CTD contains the putative NLS whereas the Spt16 subunit lacks a signal at the C-terminus. Interestingly, in Schizosaccharomyces pombe the situation is reversed since there Spt16-CTD contains the NLS and Pob3 is lacking it. This phenomena might explain why Lejeune et al.162 could generate an entire Pob3 deletion in Schizosaccharomyces pombe while Pob3 is at the same time essential in Saccharomyces cerevisiae ${ }^{158}$.

Despite the presence of the NLS on yPob3, especially its position in close proximity to the acidic residues is particularly interesting since it might influence histone binding behavior in the presence of the nuclear import machinery. Similar NLS presence in close proximity to acidic regions is also known for the different members of the Nucleoplasmin/Nucleophosmin histone chaperone family 112 . In general, all four core histones possess an NLS sequence in the N-terminal region which enables them to be imported by several importin-mediated pathways 320 . Nevertheless, some histone chaperones are identified as nucleocytoplasmic shuttles supporting nuclear import of histones. The classic example is the histone chaperone Nap1 containing a classical NLS which supports the import of H2A-H2B dimers to the nucleus 132 . For the shuttling process, yNap1 contains additionally a NES (nuclear export signal) ${ }^{321}$ which is found to be masked by its own accessory domain in a recent crystal structure ${ }^{121}$. Therefore, it might be that the masking and unmasking of the NES 
influences Nap1 localization and thereby a regulation of these shuttle processes can occur $^{322}$. Apart from the specialized shuttling chaperones it is hypothesized that maybe several chaperones participate in hitchhiking during histone import even though it would be a single ride ${ }^{320}$. Therefore, I tested whether the FACT complex subunit Pob3 can interact with the histone H2A-H2B dimer in the presence of an import receptor. I purified human importin- $\alpha$ recombinantly from Escherichia coli lacking the auto-inhibitory importin- $\beta$-binding domain (figure 3.28). Moreover, I showed that under physiological conditions a stable complex between importin- $\alpha$ and full-length ctPob3 is formed (figure 3.29). In a competitive crosslinking assay including importin- $\alpha$, ctPob3S496pBPA and histone H2A-H2B dimers, I could show that the presence of importin- $\alpha$ significantly decreases crosslink of ctPob3 to the dimer (figure 3.30). Hence, the interaction of Pob3 with the import receptor impairs dimer interaction at the acidic residues. Therefore, it is not likely that during nuclear import of Pob3 a H2A-H2B dimer is stably associated at the CTD of Pob3. At this point it should be noted that I can not exclude a histone import behavior of the FACT complex in general. Nevertheless, I can hypothesize that masking of the acidic residues due to importin binding plays an crucial role for FACT regulation. In other words, the Ran-GTP triggered disassembly of the import complex in the nucleus, thereby releasing FACT, might promote binding of FACT to chromatin exactly at its working spot. To address this question it must be first clarified, whether the FACT complex is imported as a whole entity or as single subunits.

The NLS-studies had huge implications for subsequent experiments analyzing whether the acidic residues are essential for a functional FACT complex in vivo. I performed Pob3 deletion experiments using the homologous recombination machinery in Saccharomyces cerevisiae (figure 3.31 and table 3.1). In all cases, I left the identified NLS (amino acids 544-552) untouched. The first deletion was selected in respect to a lethal Q458-stop mutation described by Schlesinger and Formosa 182 . Surprisingly, surviving yeast cells with this truncation ( $\triangle \mathrm{Q} 458-\mathrm{E} 543)$ possessed both copies, full-length and truncated Pob3, at the genomic sequence level verified by PCR (figure 3.31 A.). This might be explained either by a unusual recombination event or by spontaneous gene-duplication in yeast which is reported with a mutation rate of $\sim 0.3 \times 10^{-10}$ in haploid strains ${ }^{323 / 324}$. This is supported by the low amount of observed transformants in comparison to a control integration. Moreover, the crystal structure of Pob3 (pdb: 2GCL) suggests that a deletion from Q458 removes the capping helix of the second PH-domain of the Pob3-MD (see figure 1.10). This confirms that lethality is caused rather by structural implications and not by a deletion of the acidic residues. However, the other deletions ( $\Delta$ S491-E543 and $\Delta$ A501-E543) 
are viable and contain only the truncated version which was verified by PCR on the genomic level and by sequencing (figure 3.31 B.). Hence, a huge deletion of the acidic residues is not detrimental while leaving the putative NLS intact.

The created deletion strains ( $\triangle$ S491-E543 and $\Delta$ A501-E543) were screened for growth defects in the presence of agents which induce genotoxic and replicative stress (figure 3.32). Hydroxyurea (HU) inhibits ribonucleotide reductase and diminishes the free dNTP pool, thus, impairing processes of active DNA synthesis such as replication and can ultimately lead to double strand breaks 217 . Some of the Pob3 temperature sensitive mutants isolated by Schlesinger and Formosa, e.g. pob3L78R, showed increased HU-sensitivity which lead to the discovery that yPob3 is involved in DNA replication 182 . The authors additionally showed that Pob3 mutants are dependent on the S-phase checkpoint control triggered by MEC1. In my assays, I used the pob3L78R strain as a control for my growth assays which showed as expected temperature- and HU-sensitivity comparable to the literature ${ }^{182}$. My generated CTD deletion strains showed no observable temperature sensitive phenotype. However in the presence of hydroxyurea the deletion and control integration strains showed reduced growth compared to the wild-type BY4741 strain. This might be an artifact of the integration construct. For integration, I used a linearized pRS306 vector which contained a mutated or the wild-type Pob3 fragment. The pRS306 vector has no transcriptional terminator, thus, the integrated construct might not be efficiently processed leading to reduced Pob3 protein levels in all integration strains. This might explain the reduced growth phenotype for both, the deletion and control integration strains. Nevertheless, I can observe a distinct increased HU-sensitivity for the $\Delta$ S491-E543 strains as compared to the $\Delta$ A501-E543 and control integration strains (figure 3.32 A. and B.). This indicates that the additional deletion of 10 more amino acids (S491-S500) of the $\Delta$ S491-E543 strains is sufficient to induce mild but distinct hydroxyurea sensitivity. This clearly shows that this part of the CTD is involved during DNA replication. Unfortunately, I do not possess a yeast strain allowing me to test for the Spt ${ }^{-}$which indicates defects during transcription 182/218/219|220.

MMS (methyl methanesulfonate) is a DNA-damage inducing agent methylating guanine and adenine which can induce mutations and results in stalled replication forks $301 / 302$. Growth of deletions strains and wild-type BY4741 showed no growth defects at $32^{\circ} \mathrm{C}$ in the presence of $0.01 \%$ MMS whereas at $25^{\circ} \mathrm{C}$ the BY4741 strains showed a slight defect which might have been an technical issue. Growth on plates containing a final concentration of $0.03 \%$ MMS was abolished at $25^{\circ} \mathrm{C}$ and showed severe growth defects at $32^{\circ} \mathrm{C}$. Nevertheless, no difference in growth of the tested deletions strains and wild-type strains was detected. However, the pob3L78R strain showed severe growth effects at both temperatures and concentrations of MMS. This 
is in stark contrast to the literature where it is stated that "neither spt16-G132D nor any of the pob3 alleles...lead to sensitivity to DNA damage using MMS or UV"182.

In conclusion, I could show that acidic residues of the Pob3 CTD are not essential for cell viability but might be involved in DNA replication since the deletion strain ( $\triangle$ S491-E543) shows mild HU-sensitivity.

\subsection{Conclusions and perspectives}

With this thesis, I gained a detailed view on the molecular interactions of the histone chaperone complex FACT in Saccharomyces cerevisiae. The major findings are summarized in figure 4.1. I developed and optimized an in vivo crosslinking approach using genetically encoded crosslinker amino acids and revealed that the acidic C-terminal domain of the FACT complex subunit Pob3 interacts with the histones H2A/H2B in a defined manner. In an in vitro crosslinking approach, I confirmed these interactions using reconstituted $\mathrm{H} 2 \mathrm{~A}-\mathrm{H} 2 \mathrm{~B}$ dimers and mononucleosomes. Additionally, I characterized a novel NLS in C-terminal domain. I confirmed the NLS by fluorescence microscopy studies in vivo and interaction studies between recombinantly expressed and purified human importin- $\alpha$ and ctPob3 in vitro. Thus, claiming a bifunctional domain-character of the Pob3-CTD for nuclear transport and histone binding in baker's yeast. Furthermore, I could generate viable yeast deletion mutants, which are lacking a substantial amount of this acidic domain while leaving the previously uncharacterized NLS intact. The deletion strains ( $\triangle$ S491-E543) showed sensitivity to hydroxyurea, thereby indicating a putative implication of the Pob3 C-terminal acidic domain in DNA replication.

I observed a plethora of site-specific interactions of the histone chaperone complex FACT in vivo. Thus far, I have identified histone interactions at the CTD of Pob3 using molecular shift assays. The near future of the in vivo crosslinking approach must be seen in combination with recent proteomic approaches. Isolation of crosslinks and subsequent mass-spectrometric analysis will allow me to identify unknown interactions and map known interaction partners of the FACT complex. Moreover, a combined SILAC-crosslinking approach would allow me to quantify crosslinks, thus, distinguish direct binders at a distinct site from non-covalent associated proteins as recently shown by Hino et al. 276 . In addition to the classic SILAC approach, an isotopically labeled pBPA crosslinker amino acid could be used in quantitative proteomics approaches 325 . These techniques would allow me to site-specifically identify FACT complex interaction partners out of the in vivo approach in an unbiased comprehensive manner. 


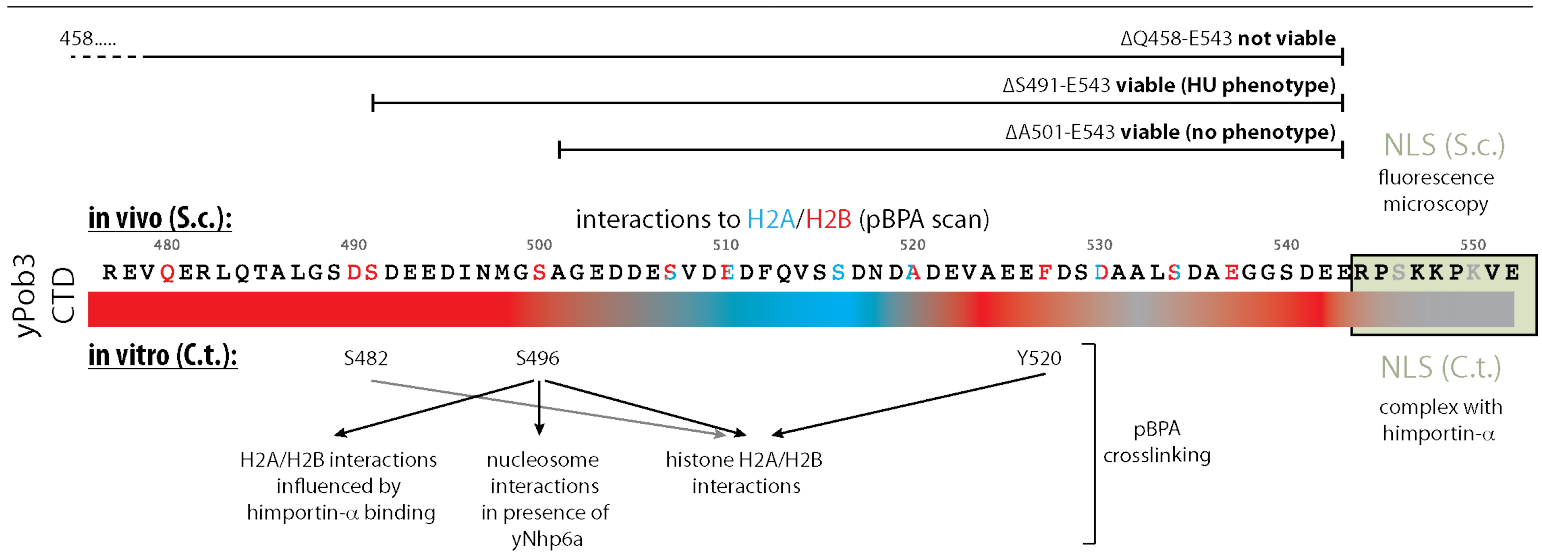

Figure 4.1: Overview of structural and functional insight of the Pob3-CTD - The yPob3-CTD was structurally and functionally characterized in vitro and in vivo. The primary sequence of yPob3-CTD and the resulting color-coded interaction profile to histones $\mathrm{H} 2 \mathrm{~A}$ and $\mathrm{H} 2 \mathrm{~B}$ is depicted in the center of the figure. Genomic deletion constructs are shown at the top whereas in vitro interaction data is located at the bottom of the figure. The characterized NLS in shown in green. For details see text.

The generated library of amber mutants is a rich source for several different applications using Genetic code expansion. At positions where an incorporated pBPA might perturb interactions or resulted in reduced full-length protein levels, I could incorporate different crosslinker amino acids such as $\mathrm{pAzF}$ or diazirine-derivatives which might improve the crosslinking ability $253 / 326$. Moreover, the Genetic code expansion strategy is not restricted to UV-inducible crosslinker amino acids 232 . The incorporation of photo-caged amino acids at critical positions ${ }^{327}$ might allow me to optochemically control FACT-activity in vivo. Additionally, I could incorporate chemical handles such as norbornene-containing amino acids which can be used for in vivo labeling with tetrazine-conjugated fluorescent dyes 249 .

Interactions can be extremely short-lived and dependent upon the cellular stage and context. Therefore, these interactions might be difficult to trap with the crosslinking approach in unsynchronized cultured cells under normal growth conditions. Thus, I could analyze interactions in synchronized yeast cells to decipher these interactions in a time-resolved manner. Additionally, I could perform crosslinking in temperature-sensitive yeast strains or in the presence of drugs to induce cellular stress. A comparative approach would allow me to investigate interactions and put them into the context of cellular processes.

In order to further investigate the biological function of the Pob3-CTD in more detail, I can use the generated Pob3-CTD deletion strain in a Synthetic Genetic Array analysis (SGA) 2821328. This high-throughput semi-automated approach would allow me to elucidate genetic interactions of my Pob3 mutant and might precisely assign the importance of the lacking acidic residues to different cellular functions.

In respect to structural biology, the ultimate goal consists of a full three-dimensional 
model of the FACT complex either in its holo-form or bound to a nucleosome. Especially the use of the thermophilic eukaryote Chaetomium thermophilum might enhance structure determination techniques such as X-ray crystallography or Cryo-electron microscopy and tomography. 


\section{Bibliography}

[1] Adam Ben-Shem, Nicolas Garreau de Loubresse, Sergey Melnikov, Lasse Jenner, Gulnara Yusupova, and Marat Yusupov. The structure of the eukaryotic ribosome at $3.0 \AA$ A resolution. Science, 334(6062):1524-1529, 2011.

[2] Gulnara Yusupova and Marat Yusupov. High-resolution structure of the eukaryotic $80 \mathrm{~S}$ ribosome. Annual Review of Biochemistry, 83:467-486, 2014.

[3] R J Ellis. Macromolecular crowding: obvious but underappreciated. Trends in Biochemical Sciences (TIBS), 26(10):597-604, 2001.

[4] Huan-Xiang Zhou, Germán Rivas, and Allen P Minton. Macromolecular crowding and confinement: biochemical, biophysical, and potential physiological consequences. Annual Review of Biophysics, 37:375-397, 2008.

[5] Huan-Xiang Zhou. Influence of crowded cellular environments on protein folding, binding, and oligomerization: Biological consequences and potentials of atomistic modeling. FEBS Letters, 587(8):1053-1061, 2013.

[6] Zachary A Gurard-Levin, Jean-Pierre Quivy, and Geneviève Almouzni. Histone Chaperones: assisting histone traffic and nucleosome dynamics. Annual Review of Biochemistry, 83(1):487-517, 2014.

[7] Aaron Klug. The discovery of the DNA double helix. Journal of Molecular Biology, 335(1):3-26, 2004.

[8] J D Watson. The double helix; a personal account of the discovery of the structure of DNA. Atheneum, 1968.

[9] Ralf Dahm. Discovering DNA: Friedrich Miescher and the early years of nucleic acid research. Human Genetics, 122(6):565-581, 2008.

[10] F Griffith. The Significance of Pneumococcal Types. The Journal of Hygiene, 27(2):113-159, 1928.

[11] O T Avery, C M Macleod, and M McCarty. Studies on the chemical nature of the substance inducing transformation of pneumococcal types: induction of transformation by a desoxyribonucleic acid fraction isolated from Pneumococcus type III. Journal of Experimental Medicine, 79(2):137-158, 1944.

[12] J D Watson and F H Crick. Molecular structure of nucleic acids; a structure for deoxyribose nucleic acid. Nature, 171(4356):737-738, 1953. 
[13] D M Brown and A R Todd. 12. Nucleotides. Part IX. The synthesis of adenylic acids a and b from 5'-trityl adenosine. Journal of the Chemical Society, 1952.

[14] Tamara L Caterino and Jeffrey J Hayes. Chromatin structure depends on what's in the nucleosome's pocket. Nature Structural \& Molecular Biology, 14(11):1056-1058, 2007.

[15] International Human Genome Sequencing Consortium. Finishing the euchromatic sequence of the human genome. Nature, 431(7011):931-945, 2004.

[16] Albrecht Kossel. The protamines and histones. Longmans Green And Company, 1928.

[17] K Luger, A W Mäder, R K Richmond, D F Sargent, and T J Richmond. Crystal structure of the nucleosome core particle at 2.8 A resolution. Nature, 389(6648):251-260, 1997.

[18] Karolin Luger. Nucleosomes: Structure and Function. eLS, 2001.

[19] Sean W Harshman, Nicolas L Young, Mark R Parthun, and Michael A Freitas. H1 histones: current perspectives and challenges. Nucleic Acids Research, 41(21):9593-9609, 2013.

[20] R E Dickerson, D S Goodsell, and S Neidle. "...the tyranny of the lattice...". Proceedings of the National Academy of Sciences, 91(9):3579-3583, 1994.

[21] Bin Wu, Kareem Mohideen, Dileep Vasudevan, and Curt A Davey. Structural insight into the sequence dependence of nucleosome positioning. Structure, 18(4):528-536, 2010.

[22] Cizhong Jiang and B Franklin Pugh. Nucleosome positioning and gene regulation: advances through genomics. Nature reviews. Genetics, 10(3):161-172, 2009.

[23] Travis N Mavrich, Ilya P Ioshikhes, Bryan J Venters, Cizhong Jiang, Lynn P Tomsho, Ji Qi, Stephan C Schuster, Istvan Albert, and B Franklin Pugh. A barrier nucleosome model for statistical positioning of nucleosomes throughout the yeast genome. Genome Research, 18(7):10731083, 2008.

[24] Guo-Cheng Yuan, Yuen-Jong Liu, Michael F Dion, Michael D Slack, Lani F Wu, Steven J Altschuler, and Oliver J Rando. Genome-scale identification of nucleosome positions in S. cerevisiae. Science, 309(5734):626-630, 2005.

[25] Eran Segal, Yvonne Fondufe-Mittendorf, Lingyi Chen, AnnChristine Thåström, Yair Field, Irene K Moore, Ji-Ping Z Wang, and Jonathan Widom. A genomic code for nucleosome positioning. Nature, 442(7104):772-778, 2006.

[26] Noam Kaplan, Irene K Moore, Yvonne Fondufe-Mittendorf, Andrea J Gossett, Desiree Tillo, Yair Field, Emily M LeProust, Timothy R Hughes, Jason D Lieb, Jonathan Widom, and Eran Segal. The DNA-encoded nucleosome organization of a eukaryotic genome. Nature, 458(7236):362-366, 2008.

[27] Yong Zhang, Zarmik Moqtaderi, Barbara P Rattner, Ghia Euskirchen, Michael Snyder, James T Kadonaga, X Shirley Liu, and Kevin Struhl. Evidence against a genomic code for nucleosome positioning. Nature Structural \& Molecular Biology, 17(8):920-923, 2010. 
[28] Paul B Talbert and Steven Henikoff. Environmental responses mediated by histone variants. Trends in Cell Biology, 24(11):642-650, 2014.

[29] Christopher M Weber and Steven Henikoff. Histone variants: dynamic punctuation in transcription. Genes \& Development, 28(7):672-682, 2014.

[30] Song Tan and Curt A Davey. Nucleosome structural studies. Current Opinion in Structural Biology, 21(1):128-136, 2011.

[31] Tatiana A Soboleva, Maxim Nekrasov, Anuj Pahwa, Rohan Williams, Gavin A Huttley, and David J Tremethick. A unique H2A histone variant occupies the transcriptional start site of active genes. Nature Structural \& Molecular Biology, 19(1):25-30, 2012.

[32] Elizabeth Sarcinella, Philip C Zuzarte, Priscilla N I Lau, Ryan Draker, and Peter Cheung. Monoubiquitylation of H2A.Z distinguishes its association with euchromatin or facultative heterochromatin. Molecular and Cellular Biology, 27(18):6457-6468, 2007.

[33] Marc D Meneghini, Michelle Wu, and Hiten D Madhani. Conserved histone variant H2A.Z protects euchromatin from the ectopic spread of silent heterochromatin. Cell, 112(5):725-736, 2003.

[34] Robert K Suto, Michael J Clarkson, David J Tremethick, and Karolin Luger. Crystal structure of a nucleosome core particle containing the variant histone H2A.Z. Nature Structural \& Molecular Biology, 7(12):1121-1124, 2000.

[35] Jun Y Fan, Danny Rangasamy, Karolin Luger, and David J Tremethick. H2A.Z alters the nucleosome surface to promote HP1alpha-mediated chromatin fiber folding. Molecular Cell, 16(4):655-661, 2004.

[36] Srinivas Chakravarthy, Sampath Kumar Y Gundimella, Cecile Caron, Pierre-Yves Perche, John R Pehrson, Saadi Khochbin, and Karolin Luger. Structural characterization of the histone variant macroH2A. Molecular and Cellular Biology, 25(17):7616-7624, 2005.

[37] P Y Perche, C Vourc'h, L Konecny, C Souchier, M Robert-Nicoud, S Dimitrov, and S Khochbin. Higher concentrations of histone macroH2A in the Barr body are correlated with higher nucleosome density. Current Biology, 10(23):1531-1534, 2000.

[38] S Chakravarthy, Y Bao, V A Roberts, D Tremethick, and K Luger. Structural characterization of histone H2A variants. Cold Spring Harbor Symposia on Quantitative Biology, 69:227-234, 2004.

[39] A J Morrison and X Shen. DNA repair in the context of chromatin. Cell Cycle, 4(4):513-512, 2005.

[40] Emmy P Rogakou, Duane R Pilch, Ann H Orr, Vessela S Ivanova, and William M Bonner. DNA double-stranded breaks induce histone H2AX phosphorylation on Serine 139. Journal of Biological Chemistry, 273(10):5858-5868, 1998.

[41] J Yuan, R Adamski, and J Chen. Focus on histone variant H2AX: to be or not to be. FEBS Letters, 584(17):3717-3724, 2010.

[42] Steven Henikoff and Takehito Furuyama. The unconventional structure of centromeric nucleosomes. Chromosoma, 121(4):341-352, 2012. 
[43] Hiroaki Tachiwana, Wataru Kagawa, Tatsuya Shiga, Akihisa Osakabe, Yuta Miya, Kengo Saito, Yoko Hayashi-Takanaka, Takashi Oda, Mamoru Sato, Sam-Yong Park, Hiroshi Kimura, and Hitoshi Kurumizaka. Crystal structure of the human centromeric nucleosome containing CENP-A. Nature, 476(7359):232-235, 2011.

[44] Peter E Warburton, Carol A Cooke, Sylvie Bourassa, Omid Vafa, Beth A Sullivan, Gail Stetten, Giorgio Gimelli, Dorothy Warburton, Chris Tyler-Smith, Kevin F Sullivan, Guy G Poirier, and William C Earnshaw. Immunolocalization of CENP-A suggests a distinct nucleosome structure at the inner kinetochore plate of active centromeres. Current Biology, 7(11):901-904, 1997.

[45] Aaron A Van Hooser, Ilia I Ouspenski, Heather C Gregson, Daniel A Starr, Tim J Yen, Michael L Goldberg, Kyoko Yokomori, William C Earnshaw, Kevin F Sullivan, and B R Brinkley. Specification of kinetochore-forming chromatin by the histone H3 variant CENP-A. Journal of Cell Science, 114(19):3529-3542, 2001.

[46] Dan Filipescu, Emmanuelle Szenker, and Geneviève Almouzni. Developmental roles of histone H3 variants and their chaperones. Trends in Genetics (TIG), 29(11):630-640, 2013.

[47] Hideaki Tagami, Dominique Ray-Gallet, Geneviève Almouzni, and Yoshihiro Nakatani. Histone H3.1 and H3.3 complexes mediate nucleosome assembly pathways dependent or independent of DNA synthesis. Cell, 116(1):51-61, 2004.

[48] Sukesh R Bhaumik, Edwin Smith, and Ali Shilatifard. Covalent modifications of histones during development and disease pathogenesis. Nature Structural \& Molecular Biology, 14(11):1008-1016, 2007.

[49] Aaron D Goldberg, C David Allis, and Emily Bernstein. Epigenetics: a landscape takes shape. Cell, 128(4):635-638, 2007.

[50] Catherine A Musselman, Marie-Eve Lalonde, Jacques Côté, and Tatiana G Kutateladze. Perceiving the epigenetic landscape through histone readers. Nature Structural \& Molecular Biology, 19(12):1218-1227, 2012.

[51] Minjia Tan, Hao Luo, Sangkyu Lee, Fulai Jin, Jeong Soo Yang, Emilie Montellier, Thierry Buchou, Zhongyi Cheng, Sophie Rousseaux, Nisha Rajagopal, Zhike Lu, Zhen Ye, Qin Zhu, Joanna Wysocka, Yang Ye, Saadi Khochbin, Bing Ren, and Yingming Zhao. Identification of 67 histone marks and histone lysine crotonylation as a new type of histone modification. Cell, 146(6):1016-1028, 2011.

[52] Yue Chen, Robert Sprung, Yi Tang, Haydn Ball, Bhavani Sangras, Sung Chan Kim, John R Falck, Junmin Peng, Wei Gu, and Yingming Zhao. Lysine propionylation and butyrylation are novel post-translational modifications in histones. Molecular \& Cellular Proteomics, 6(5):812-819, 2007.

[53] Miyong Yun, Jun $\mathrm{Wu}$, Jerry L Workman, and Bing Li. Readers of histone modifications. Cell Research, 21(4):564-578, 2011.

[54] Dinshaw J Patel and Zhanxin Wang. Readout of epigenetic modifications. Annual Review of Biochemistry, 82:81-118, 2013. 
[55] Oliver J Rando and Fred Winston. Chromatin and transcription in yeast. Genetics, 190(2):351-387, 2012.

[56] S Y Roth and C D Allis. Chromatin condensation: does histone H1 dephosphorylation play a role? Trends in Biochemical Sciences (TIBS), 17(3):93-98, 1992.

[57] Bradley E Bernstein, Michael Kamal, Kerstin Lindblad-Toh, Stefan Bekiranov, Dione K Bailey, Dana J Huebert, Scott McMahon, Elinor K Karlsson, Edward J Kulbokas, Thomas R Gingeras, Stuart L Schreiber, and Eric S Lander. Genomic maps and comparative analysis of histone modifications in human and mouse. Cell, 120(2):169-181, 2005.

[58] Alexander J Ruthenburg, C David Allis, and Joanna Wysocka. Methylation of lysine 4 on histone H3: intricacy of writing and reading a single epigenetic mark. Molecular Cell, 25(1):15-30, 2007.

[59] Joomyeong Kim and Hana Kim. Recruitment and biological consequences of histone modification of H3K27me3 and H3K9me3. ILAR Journal, 53(3-4):232-239, 2012.

[60] Jiansheng Zhou, Jun Y Fan, Danny Rangasamy, and David J Tremethick. The nucleosome surface regulates chromatin compaction and couples it with transcriptional repression. Nature Structural \& Molecular Biology, 14(11):1070-1076, 2007.

[61] Curt A Davey, David F Sargent, Karolin Luger, Armin W Maeder, and Timothy J Richmond. Solvent mediated interactions in the structure of the nucleosome core particle at $1.9 \AA$ resolution. Journal of Molecular Biology, 319(5):1097-1113, 2002.

[62] Benedetta Dorigo, Thomas Schalch, Alexandra Kulangara, Sylwia Duda, Rasmus R Schroeder, and Timothy J Richmond. Nucleosome arrays reveal the two-start organization of the chromatin fiber. Science, 306(5701):1571-1573, 2004.

[63] Bryan J Wilkins, Nils A Rall, Yogesh Ostwal, Tom Kruitwagen, Kyoko Hiragami-Hamada, Marco Winkler, Yves Barral, Wolfgang Fischle, and Heinz Neumann. A cascade of histone modifications induces chromatin condensation in mitosis. Science, 343(6166):77-80, 2014.

[64] Michael Shogren-Knaak, Haruhiko Ishii, Jian-Min Sun, Michael J Pazin, James R Davie, and Craig L Peterson. Histone H4-K16 acetylation controls chromatin structure and protein interactions. Science, 311(5762):844-847, 2006.

[65] B D Strahl and C D Allis. The language of covalent histone modifications. Nature, 403(6765):41$45,2000$.

[66] Wolfgang Fischle, Yanming Wang, and C David Allis. Histone and chromatin cross-talk. Current Opinion in Cell Biology, 15(2):172-183, 2003.

[67] John A Latham and Sharon Y R Dent. Cross-regulation of histone modifications. Nature Structural \& Molecular Biology, 14(11):1017-1024, 2007.

[68] K J Polach and J Widom. Mechanism of protein access to specific DNA sequences in chromatin: a dynamic equilibrium model for gene regulation. Journal of Molecular Biology, 254(2):130-149, 1995. 
[69] Gu Li, Marcia Levitus, Carlos Bustamante, and Jonathan Widom. Rapid spontaneous accessibility of nucleosomal DNA. Nature Structural \& Molecular Biology, 12(1):46-53, 2005.

[70] W J A Koopmans, A Brehm, C Logie, T Schmidt, and J van Noort. Single-pair FRET microscopy reveals mononucleosome dynamics. Journal of Fluorescence, 17(6):785-795, 2007.

[71] Vera Böhm, Aaron R Hieb, Andrew J Andrews, Alexander Gansen, Andrea Rocker, Katalin Tóth, Karolin Luger, and Jörg Langowski. Nucleosome accessibility governed by the dimer/tetramer interface. Nucleic Acids Research, 39(8):3093-3102, 2011.

[72] Lloyd Davis and Jason W Chin. Designer proteins: applications of genetic code expansion in cell biology. Nature Reviews Molecular Cell Biology, 13(3):168-182, 2012.

[73] Andrew J Andrews and Karolin Luger. Nucleosome structure(s) and stability: variations on a theme. Annual Review of Biophysics, 40(1):99-117, 2011.

[74] Karolin Luger, Mekonnen L Dechassa, and David J Tremethick. New insights into nucleosome and chromatin structure: an ordered state or a disordered affair? Nature Reviews Molecular Cell Biology, 13(7):436-447, 2012.

[75] Heinz Neumann, Susan M Hancock, Ruth Buning, Andrew Routh, Lynda Chapman, Joanna Somers, Tom Owen-Hughes, John van Noort, Daniela Rhodes, and Jason W Chin. A method for genetically installing site-specific acetylation in recombinant histones defines the effects of H3 K56 acetylation. Molecular Cell, 36(1):153-163, 2009.

[76] J Ausio, D Seger, and H Eisenberg. Nucleosome core particle stability and conformational change. Effect of temperature, particle and $\mathrm{NaCl}$ concentrations, and crosslinking of histone $\mathrm{H} 3$ sulfhydryl groups. Journal of Molecular Biology, 176(1):77-104, 1984.

[77] Brent D Brower-Toland, Corey L Smith, Richard C Yeh, John T Lis, Craig L Peterson, and Michelle D Wang. Mechanical disruption of individual nucleosomes reveals a reversible multistage release of DNA. Proceedings of the National Academy of Sciences, 99(4):1960-1965, 2002.

[78] Atsushi Miyagi, Toshio Ando, and Yuri L Lyubchenko. Dynamics of nucleosomes assessed with time-lapse high-speed atomic force microscopy. Biochemistry, 50(37):7901-7908, 2011.

[79] Christopher W Akey and Karolin Luger. Histone chaperones and nucleosome assembly. Current Opinion in Structural Biology, 13(1):6-14, 2003.

[80] K Luger, T J Rechsteiner, and T J Richmond. Preparation of nucleosome core particle from recombinant histones. Methods in Enzymology, 304:3-19, 1999.

[81] Pamela N Dyer, Raji S Edayathumangalam, Cindy L White, Yunhe Bao, Srinivas Chakravarthy, Uma M Muthurajan, and Karolin Luger. Reconstitution of nucleosome core particles from recombinant histones and DNA. Methods in Enzymology, 375:23-44, 2004.

[82] Rimma Belotserkovskaya, Sangtaek Oh, Vladimir A Bondarenko, George Orphanides, Vasily M Studitsky, and Danny Reinberg. FACT facilitates transcription-dependent nucleosome alteration. Science, 301(5636):1090-1093, 2003. 
[83] Maria L Kireeva, Wendy Walter, Vladimir Tchernajenko, Vladimir Bondarenko, Mikhail Kashlev, and Vasily M Studitsky. Nucleosome remodeling induced by RNA Polymerase II. Molecular Cell, 9(3):541-552, 2002.

[84] Yasuhiro Arimura, Hiroaki Tachiwana, Takashi Oda, Mamoru Sato, and Hitoshi Kurumizaka. Structural analysis of the hexasome, lacking one histone $\mathrm{H} 2 \mathrm{~A} / \mathrm{H} 2 \mathrm{~B}$ dimer from the conventional nucleosome. Biochemistry, 51(15):3302-3309, 2012.

[85] H Kimura and P R Cook. Kinetics of core histones in living human cells: little exchange of H3 and H4 and some rapid exchange of H2B. The Journal of Cell Biology, 153(7):1341-1353, 2001.

[86] Adil Jamai, Rachel Maria Imoberdorf, and Michel Strubin. Continuous histone H2B and transcription-dependent histone $\mathrm{H} 3$ exchange in yeast cells outside of replication. Molecular Cell, 25(3):345-355, 2007.

[87] Cedric R Clapier and Bradley R Cairns. The biology of chromatin remodeling complexes. Annual Review of Biochemistry, 78:273-304, 2009.

[88] Peter B Becker and Wolfram Hörz. ATP-dependent nucleosome remodeling. Annual Review of Biochemistry, 71:247-273, 2002.

[89] Felix Mueller-Planitz, Henrike Klinker, and Peter B Becker. Nucleosome sliding mechanisms: new twists in a looped history. Nature Structural \& Molecular Biology, 20(9):1026-1032, 2013.

[90] Sebastian Deindl, William L Hwang, Swetansu K Hota, Timothy R Blosser, Punit Prasad, Blaine Bartholomew, and Xiaowei Zhuang. ISWI remodelers slide nucleosomes with coordinated multi-base-pair entry steps and single-base-pair exit steps. Cell, 152(3):442-452, 2013.

[91] Geeta J Narlikar, Ramasubramanian Sundaramoorthy, and Tom Owen-Hughes. Mechanisms and functions of ATP-dependent chromatin-remodeling enzymes. Cell, 154(3):490-503, 2013.

[92] Haiying Zhang, Douglas N Roberts, and Bradley R Cairns. Genome-wide dynamics of Htz1, a histone $\mathrm{H} 2 \mathrm{~A}$ variant that poises repressed/basal promoters for activation through histone loss. Cell, 123(2):219-231, 2005.

[93] Gaku Mizuguchi, Xuetong Shen, Joe Landry, Wei-Hua Wu, Subhojit Sen, and Carl Wu. ATPdriven exchange of histone $\mathrm{H} 2 \mathrm{AZ}$ variant catalyzed by SWR1 chromatin remodeling complex. Science, 303(5656):343-348, 2004.

[94] Concetta G A Marfella and Anthony N Imbalzano. The Chd family of chromatin remodelers. Mutation Research, 618(1-2):30-40, 2007.

[95] Manolis Papamichos-Chronakis, Shinya Watanabe, Oliver J Rando, and Craig L Peterson. Global regulation of H2A.Z localization by the INO80 chromatin-remodeling enzyme is essential for genome integrity. Cell, 144(2):200-213, 2011.

[96] Joseph A Goldman, Joseph D Garlick, and Robert E Kingston. Chromatin remodeling by imitation switch (ISWI) class ATP-dependent remodelers is stimulated by histone variant H2A.Z. Journal of Biological Chemistry, 285(7):4645-4651, 2010. 
[97] R A Laskey, B M Honda, A D Mills, and J T Finch. Nucleosomes are assembled by an acidic protein which binds histones and transfers them to DNA. Nature, 275(5679):416-420, 1978.

[98] William C Earnshaw, Barry M Honda, Ronald A Laskey, and Jean O Thomas. Assembly of nucleosomes: the reaction involving X. laevis nucleoplasmin. Cell, 21(2):373-383, 1980.

[99] Rebecca J Burgess and Zhiguo Zhang. Histone chaperones in nucleosome assembly and human disease. Nature Structural \& Molecular Biology, 20(1):14-22, 2013.

[100] Dan Su, Qi Hu, Qing Li, James R Thompson, Gaofeng Cui, Ahmed Fazly, Brian A Davies, Maria Victoria Botuyan, Zhiguo Zhang, and Georges Mer. Structural basis for recognition of H3K56-acetylated histone H3-H4 by the chaperone Rtt106. Nature, 483(7387):104-107, 2012.

[101] Sheena D'Arcy Simon J Elsässer. Towards A Mechanism for Histone Chaperones. Biochimica et Biophysica Acta, 1819(0):211-221, 2012.

[102] Susan Smith and Bruce Stillman. Purification and characterization of CAF-I, a human cell factor required for chromatin assembly during DNA replication in vitro. Cell, 58(1):15-25, 1990.

[103] Dominique Ray-Gallet, Jean-Pierre Quivy, Christine Scamps, Emmanuelle M-D Martini, Marc Lipinski, and Geneviève Almouzni. HIRA is critical for a nucleosome assembly pathway independent of DNA synthesis. Molecular Cell, 9(5):1091-1100, 2002.

[104] Elaine M Dunleavy, Danièle Roche, Hideaki Tagami, Nicolas Lacoste, Dominique Ray-Gallet, Yusuke Nakamura, Yataro Daigo, Yoshihiro Nakatani, and Geneviève Almouzni-Pettinotti. HJURP is a cell-cycle-dependent maintenance and deposition factor of CENP-A at centromeres. Cell, 137(3):485-497, 2009.

[105] Daniel R Foltz, Lars E T Jansen, Aaron O Bailey, John R Yates, Emily A Bassett, Stacey Wood, Ben E Black, and Don W Cleveland. Centromere-specific assembly of CENP-A nucleosomes is mediated by HJURP. Cell, 137(3):472-484, 2009.

[106] Adam J L Cook, Zachary A Gurard-Levin, Isabelle Vassias, and Geneviève Almouzni. A specific function for the histone chaperone NASP to fine-tune a reservoir of soluble $\mathrm{H} 3-\mathrm{H} 4$ in the histone supply chain. Molecular Cell, 44(6):918-927, 2011.

[107] J K Tyler, C R Adams, S R Chen, R Kobayashi, R T Kamakaka, and J T Kadonaga. The RCAF complex mediates chromatin assembly during DNA replication and repair. Nature, 402(6761):555-560, 1999.

[108] Y Ishimi, M Kojima, M Yamada, and F Hanaoka. Binding mode of nucleosome-assembly protein (AP-I) and histones. European Journal of Biochemistry / FEBS, 162(1):19-24, 1987.

[109] G A McQuibban, C N Commisso-Cappelli, and P N Lewis. Assembly, remodeling, and histone binding capabilities of yeast nucleosome assembly protein 1. Journal of Biological Chemistry, 273(11):6582-6590, 1998.

[110] Paul D Kaufman, Jennifer L Cohen, and Mary Ann Osley. Hir proteins are required for positiondependent gene silencing in Saccharomyces cerevisiae in the absence of Chromatin Assembly Factor I. Molecular and Cellular Biology, 18(8):4793-114, 1998. 
[111] Andrew P VanDemark, Hua Xin, Laura McCullough, Robert Rawlins, Shayla Bentley, Annie Heroux, David J Stillman, Christopher P Hill, and Tim Formosa. Structural and functional analysis of the Spt16p N-terminal domain reveals overlapping roles of yFACT subunits. Journal of Biological Chemistry, 283(8):5058-5068, 2008.

[112] M Eitoku, L Sato, T Senda, and M Horikoshi. Histone chaperones: 30 years from isolation to elucidation of the mechanisms of nucleosome assembly and disassembly. Cellular and Molecular Life Sciences, 65(3):414-444, 2007.

[113] Sally M Daganzo, Jan P Erzberger, Wendy M Lam, Emmanuel Skordalakes, Rugang Zhang, Alexa A Franco, Steven J Brill, Peter D Adams, James M Berger, and Paul D Kaufman. Structure and function of the conserved core of histone deposition protein Asf1. Current Biology, 13(24):2148-2158, 2003.

[114] Christine M English, Melissa W Adkins, Joshua J Carson, Mair E A Churchill, and Jessica K Tyler. Structural basis for the histone chaperone activity of Asf1. Cell, 127(3):495-508, 2006.

[115] Emmanuelle Szenker, Dominique Ray-Gallet, and Geneviève Almouzni. The double face of the histone variant H3.3. Cell Research, 21(3):421-434, 2011.

[116] Yong Tang, Maxim V Poustovoitov, Kehao Zhao, Megan Garfinkel, Adrian Canutescu, Roland Dunbrack, Peter D Adams, and Ronen Marmorstein. Structure of a human ASF1a-HIRA complex and insights into specificity of histone chaperone complex assembly. Nature Structural \& Molecular Biology, 13(10):921-929, 2006.

[117] Federico Abascal, Armelle Corpet, Zachary A Gurard-Levin, David Juan, Françoise Ochsenbein, Daniel Rico, Alfonso Valencia, and Geneviève Almouzni. Subfunctionalization via adaptive evolution influenced by genomic context: the case of histone chaperones ASF1a and ASF1b. Molecular Biology and Evolution, 30(8):1853-1866, 2013.

[118] J G Moggs, P Grandi, J P Quivy, Z O Jónsson, U Hübscher, P B Becker, and G Almouzni. A CAF-1-PCNA-mediated chromatin assembly pathway triggered by sensing DNA damage. Molecular and Cellular Biology, 20(4):1206-1218, 2000.

[119] Simon J Elsässer, Hongda Huang, Peter W Lewis, Jason W Chin, C David Allis, and Dinshaw J Patel. DAXX envelops a histone H3.3-H4 dimer for H3.3-specific recognition. Nature, 491(7425):560-565, 2012.

[120] Hao Hu, Yang Liu, Mingzhu Wang, Junnan Fang, Hongda Huang, Na Yang, Yanbo Li, Jianyu Wang, Xuebiao Yao, Yunyu Shi, Guohong Li, and Rui-Ming Xu. Structure of a CENP-A-histone H4 heterodimer in complex with chaperone HJURP. Genes \& Development, 25(9):901-906, 2011.

[121] Young-Jun Park and Karolin Luger. The structure of nucleosome assembly protein 1. Proceedings of the National Academy of Sciences, 103(5):1248-1253, 2006.

[122] Luke Selth and Jesper Q Svejstrup. Vps75, a new yeast member of the NAP histone chaperone family. Journal of Biological Chemistry, 282(17):12358-12362, 2007. 
[123] Andrew Bowman, Colin M Hammond, Andrew Stirling, Richard Ward, Weifeng Shang, Hassane El-Mkami, David A Robinson, Dmitri I Svergun, David G Norman, and Tom Owen-Hughes. The histone chaperones Vps75 and Nap1 form ring-like, tetrameric structures in solution. Nucleic Acids Research, 42(9):6038-6051, 2014.

[124] Klaus Scheffzek and Stefan Welti. Pleckstrin homology (PH) like domains-versatile modules in protein-protein interaction platforms. FEBS Letters, 586(17):2662-2673, 2012.

[125] Hiroshi Masumoto, David Hawke, Ryuji Kobayashi, and Alain Verreault. A role for cell-cycleregulated histone H3 lysine 56 acetylation in the DNA damage response. Nature, 436(7048):294298, 2005.

[126] Junhong Han, Hui Zhou, Bruce Horazdovsky, Kangling Zhang, Rui-Ming Xu, and Zhiguo Zhang. Rtt109 acetylates histone H3 lysine 56 and functions in DNA replication. Science, 315(5812):653-655, 2007.

[127] Junhong Han, Hui Zhou, Zhizhong Li, Rui-Ming Xu, and Zhiguo Zhang. The Rtt109-Vps75 histone acetyltransferase complex acetylates non-nucleosomal histone H3. Journal of Biological Chemistry, 282(19):14158-14164, 2007.

[128] Qing Li, Hui Zhou, Hugo Wurtele, Brian Davies, Bruce Horazdovsky, Alain Verreault, and Zhiguo Zhang. Acetylation of histone H3 lysine 56 regulates replication-coupled nucleosome assembly. Cell, 134(2):244-255, 2008.

[129] Chin-Chuan Chen, Joshua J Carson, Jason Feser, Beth Tamburini, Susan Zabaronick, Jeffrey Linger, and Jessica K Tyler. Acetylated lysine 56 on histone $\mathrm{H} 3$ drives chromatin assembly after repair and signals for the completion of repair. Cell, 134(2):231-243, 2008.

[130] Ahmed Fazly, Qing Li, Qi Hu, Georges Mer, Bruce Horazdovsky, and Zhiguo Zhang. Histone chaperone Rtt106 promotes nucleosome formation using (H3-H4)2 tetramers. Journal of Biological Chemistry, 287(14):10753-10760, 2012.

[131] Dominique Ray-Gallet, Adam Woolfe, Isabelle Vassias, Céline Pellentz, Nicolas Lacoste, Aastha Puri, David C Schultz, Nikolay A Pchelintsev, Peter D Adams, Lars E T Jansen, and Geneviève Almouzni. Dynamics of histone H3 deposition in vivo reveal a nucleosome gap-filling mechanism for H3.3 to maintain chromatin integrity. Molecular Cell, 44(6):928-941, 2011.

[132] Nima Mosammaparast, Courtney S Ewart, and Lucy F Pemberton. A role for nucleosome assembly protein 1 in the nuclear transport of histones H2A and H2B. The EMBO Journal, 21(23):6527-6538, 2002.

[133] Eric I Campos, Jeffrey Fillingham, Guohong Li, Haiyan Zheng, Philipp Voigt, Wei-Hung W Kuo, Harshika Seepany, Zhonghua Gao, Loren A Day, Jack F Greenblatt, and Danny Reinberg. The program for processing newly synthesized histones H3.1 and H4. Nature Structural \& Molecular Biology, 17(11):1343-1351, 2010.

[134] Zuzana Jasencakova, Annette N D Scharf, Katrine Ask, Armelle Corpet, Axel Imhof, Geneviève Almouzni, and Anja Groth. Replication stress interferes with histone recycling and predeposition marking of new histones. Molecular Cell, 37(5):736-743, 2010. 
[135] J P Quivy, P Grandi, and G Almouzni. Dimerization of the largest subunit of chromatin assembly factor 1: importance in vitro and during Xenopus early development. The EMBO Journal, 20(8):2015-2027, 2001.

[136] Duane D Winkler, Hui Zhou, Mohd A Dar, Zhiguo Zhang, and Karolin Luger. Yeast CAF1 assembles histone (H3-H4)2 tetramers prior to DNA deposition. Nucleic Acids Research, 40(20):10139-10149, 2012.

[137] Chandrima Das, M Scott Lucia, Kirk C Hansen, and Jessica K Tyler. CBP/p300-mediated acetylation of histone H3 on lysine 56. Nature, 459(7243):113-117, 2009.

[138] R E Sobel, R G Cook, C A Perry, A T Annunziato, and C D Allis. Conservation of depositionrelated acetylation sites in newly synthesized histones $\mathrm{H} 3$ and H4. Proceedings of the National Academy of Sciences, 92(4):1237-1241, 1995.

[139] Ryo Natsume, Masamitsu Eitoku, Yusuke Akai, Norihiko Sano, Masami Horikoshi, and Toshiya Senda. Structure and function of the histone chaperone CIA/ASF1 complexed with histones H3 and H4. Nature, 446(7133):338-341, 2007.

[140] Anja Groth, Armelle Corpet, Adam J L Cook, Danièle Roche, Jiri Bartek, Jiri Lukas, and Geneviève Almouzni. Regulation of replication fork progression through histone supply and demand. Science, 318(5858):1928-1931, 2007.

[141] Mo Xu, Chengzu Long, Xiuzhen Chen, Chang Huang, She Chen, and Bing Zhu. Partitioning of histone H3-H4 tetramers during DNA replication-dependent chromatin assembly. Science, 328(5974):94-98, 2010.

[142] Andrew P VanDemark, Mary Blanksma, Elliott Ferris, Annie Heroux, Christopher P Hill, and Tim Formosa. The structure of the yFACT Pob3-M domain, its interaction with the DNA Replication Factor RPA, and a potential role in nucleosome deposition. Molecular Cell, 22(3):363374, 2006.

[143] Bertrand Chin-Ming Tan, Cheng-Ting Chien, Susumu Hirose, and Sheng-Chung Lee. Functional cooperation between FACT and MCM helicase facilitates initiation of chromatin DNA replication. The EMBO Journal, 25(17):3975-3985, 2006.

[144] T Fujii-Nakata, Y Ishimi, A Okuda, and A Kikuchi. Functional analysis of nucleosome assembly protein, NAP-1. The negatively charged $\mathrm{COOH}$-terminal region is not necessary for the intrinsic assembly activity. Journal of Biological Chemistry, 267(29):20980-20986, 1992.

[145] Andrew J Andrews, Xu Chen, Alexander Zevin, Laurie A Stargell, and Karolin Luger. The histone chaperone Nap1 promotes nucleosome assembly by eliminating nonnucleosomal histone DNA interactions. Molecular Cell, 37(6):834-842, 2010.

[146] T Ito, M Bulger, M J Pazin, R Kobayashi, and J T Kadonaga. ACF, an ISWI-containing and ATP-utilizing chromatin assembly and remodeling factor. Cell, 90(1):145-155, 1997.

[147] Christopher R Brown, Changhui Mao, Elena Falkovskaia, Jason K Law, and Hinrich Boeger. In vivo role for the chromatin-remodeling enzyme SWI/SNF in the removal of promoter nucleosomes by disassembly rather than sliding. Journal of Biological Chemistry, 286(47):4055640565, 2011. 
[148] Yahli Lorch, Joachim Griesenbeck, Hinrich Boeger, Barbara Maier-Davis, and Roger D Kornberg. Selective removal of promoter nucleosomes by the RSC chromatin-remodeling complex. Nature Structural \& Molecular Biology, 18(8):881-885, 2011.

[149] Marc A Schwabish and Kevin Struhl. Asf1 mediates histone eviction and deposition during elongation by RNA polymerase II. Molecular Cell, 22(3):415-422, 2006.

[150] M Ransom, S K Williams, M L Dechassa, C Das, J Linger, M Adkins, C Liu, B Bartholomew, and J K Tyler. FACT and the proteasome promote promoter chromatin disassembly and transcriptional initiation. Journal of Biological Chemistry, 284(35):23461-23471, 2009.

[151] A Bortvin and F Winston. Evidence that Spt6p controls chromatin structure by a direct interaction with histones. Science, 272(5267):1473-1476, 1996.

[152] Mai Sun, Laurent Larivière, Stefan Dengl, Andreas Mayer, and Patrick Cramer. A tandem SH2 domain in transcription elongation factor Spt6 binds the phosphorylated RNA polymerase II C-terminal repeat domain (CTD). Journal of Biological Chemistry, 285(53):41597-41603, 2010.

[153] Tim Formosa. The role of FACT in making and breaking nucleosomes. Biochimica et Biophysica Acta, 1819(3-4):247-255, 2012.

[154] Maria Hondele and Andreas G Ladurner. Catch me if you can: how the histone chaperone FACT capitalizes on nucleosome breathing. Nucleus, 4(6):443-449, 2013.

[155] Duane D Winkler and Karolin Luger. The histone chaperone FACT: structural insights and mechanisms for nucleosome reorganization. Journal of Biological Chemistry, 286(21):18369-18374, 2011.

[156] Danny Reinberg and Robert J Sims. de FACTo nucleosome dynamics. Journal of Biological Chemistry, 281(33):23297-23301, 2006.

[157] A Rowley, R A Singer, and G C Johnston. CDC68, a yeast gene that affects regulation of cell proliferation and transcription, encodes a protein with a highly acidic carboxyl terminus. Molecular and Cellular Biology, 11(11):5718-5726, 1991.

[158] J Wittmeyer and T Formosa. The Saccharomyces cerevisiae DNA polymerase alpha catalytic subunit interacts with Cdc68/Spt16 and with Pob3, a protein similar to an HMG1-like protein. Molecular and Cellular Biology, 17(7):4178-4190, 1997.

[159] T Formosa, P Eriksson, J Wittmeyer, J Ginn, Y Yu, and D J Stillman. Spt16-Pob3 and the HMG protein Nhp6 combine to form the nucleosome-binding factor SPN. The EMBO Journal, 20(13):3506-3517, 2001.

[160] G Orphanides, G LeRoy, C H Chang, D S Luse, and D Reinberg. FACT, a factor that facilitates transcript elongation through nucleosomes. Cell, 92(1):105-116, 1998.

[161] G Orphanides, W H Wu, W S Lane, M Hampsey, and D Reinberg. The chromatin-specific transcription elongation factor FACT comprises human SPT16 and SSRP1 proteins. Nature, 400(6741):284-288, 1999. 
[162] Erwan Lejeune, Miriam Bortfeld, Sharon A White, Alison L Pidoux, Karl Ekwall, Robin C Allshire, and Andreas G Ladurner. The chromatin-remodeling factor FACT contributes to centromeric heterochromatin independently of RNAi. Current Biology, 17(14):1219-1224, 2007.

[163] G LeRoy, G Orphanides, W S Lane, and D Reinberg. Requirement of RSF and FACT for transcription of chromatin templates in vitro. Science, 282(5395):1900-1904, 1998.

[164] Debabrata Biswas, Yaxin Yu, Matthew Prall, Tim Formosa, and David J Stillman. The yeast FACT complex has a role in transcriptional initiation. Molecular and Cellular Biology, 25(14):5812-5822, 2005.

[165] Craig D Kaplan, Lisa Laprade, and Fred Winston. Transcription elongation factors repress transcription initiation from cryptic sites. Science, 301(5636):1096-1099, 2003.

[166] Paul B Mason and Kevin Struhl. The FACT complex travels with elongating RNA polymerase II and is important for the fidelity of transcriptional initiation in vivo. Molecular and Cellular Biology, 23(22):8323-8333, 2003.

[167] Warren P Voth, Shinya Takahata, Joy L Nishikawa, Benjamin M Metcalfe, Anders M Näär, and David J Stillman. A role for FACT in repopulation of nucleosomes at inducible genes. PLOS ONE, 9(1):e84092, 2014.

[168] Bertrand Tan, Hsuan Liu, Chih-Li Lin, and Sheng-Chung Lee. Functional cooperation between FACT and MCM is coordinated with cell cycle and differential complex formation. Journal of Biomedical Science, 17(1):11, 2010.

[169] Guillaume M Hautbergue, Ming-Lung Hung, Matthew J Walsh, Ambrosius P L Snijders, ChungTe Chang, Rachel Jones, Chris P Ponting, Mark J Dickman, and Stuart A Wilson. UIF, a New mRNA export adaptor that works together with REF/ALY, requires FACT for recruitment to mRNA. Current Biology, 19(22):1918-1924, 2009.

[170] T Abe, K Sugimura, Y Hosono, Y Takami, M Akita, A Yoshimura, S Tada, T Nakayama, H Murofushi, K Okumura, S Takeda, M Horikoshi, M Seki, and T Enomoto. The histone chaperone Facilitates Chromatin Transcription (FACT) protein maintains normal replication fork rates. Journal of Biological Chemistry, 286(35):30504-30512, 2011.

[171] David M Keller and Hua Lu. p53 serine 392 phosphorylation increases after UV through induction of the assembly of the CK2.hSPT16.SSRP1 complex. Journal of Biological Chemistry, 277(51):50206-50213, 2002.

[172] Allyson F O'Donnell, Neil K Brewster, Joelius Kurniawan, Laura V Minard, Gerald C Johnston, and Richard A Singer. Domain organization of the yeast histone chaperone FACT: the conserved $\mathrm{N}$-terminal domain of FACT subunit Spt16 mediates recovery from replication stress. Nucleic Acids Research, 32(19):5894-5906, 2004.

[173] Yasuo Tsunaka, Junko Toga, Hiroto Yamaguchi, Shin-ichi Tate, Susumu Hirose, and Kosuke Morikawa. Phosphorylated intrinsically disordered region of FACT masks its nucleosomal DNA binding elements. Journal of Biological Chemistry, 284(36):24610-24621, 2009. 
[174] Tobias Stuwe, Michael Hothorn, Erwan Lejeune, Vladimir Rybin, Miriam Bortfeld, Klaus Scheffzek, and Andreas G Ladurner. The FACT Spt16 "peptidase" domain is a histone H3-H4 binding module. Proceedings of the National Academy of Sciences, 105(26):8884-8889, 2008.

[175] Maria Hondele, Tobias Stuwe, Markus Hassler, Felix Halbach, Andrew Bowman, Elisa T Zhang, Bianca Nijmeijer, Christiane Kotthoff, Vladimir Rybin, Stefan Amlacher, Ed Hurt, and Andreas G Ladurner. Structural basis of histone H2A-H2B recognition by the essential chaperone FACT. Nature, 499(7456):111-114, 2013.

[176] David J Kemble, Frank G Whitby, Howard Robinson, Laura L McCullough, Tim Formosa, and Christopher P Hill. Structure of the Spt16 middle domain reveals functional features of the histone chaperone FACT. Journal of Biological Chemistry, 288(15):10188-10194, 2013.

[177] Rachel M Zunder, Andrew J Antczak, James M Berger, and Jasper Rine. Two surfaces on the histone chaperone Rtt106 mediate histone binding, replication, and silencing. Proceedings of the National Academy of Sciences, 2011.

[178] Suting Zheng, J Brooks Crickard, Abhinaya Srikanth, and Joseph C Reese. A highly conserved region within H2B is important for FACT to act on nucleosomes. Molecular and Cellular Biology, 34(3):303-314, 2014.

[179] John J Wyrick, McKenna N M Kyriss, and William B Davis. Ascending the nucleosome face: recognition and function of structured domains in the histone H2A-H2B dimer. Biochimica et Biophysica Acta, 1819(8):892-901, 2012.

[180] D R Evans, N K Brewster, Q Xu, A Rowley, B A Altheim, G C Johnston, and R A Singer. The yeast protein complex containing Cdc68 and Pob3 mediates core-promoter repression through the Cdc68 N-terminal domain. Genetics, 150(4):1393-1405, 1998.

[181] D D Winkler, U M Muthurajan, A R Hieb, and K Luger. Histone chaperone FACT coordinates nucleosome interaction through multiple synergistic binding events. Journal of Biological Chemistry, 286(48):41883-41892, 2011.

[182] M B Schlesinger and T Formosa. POB3 is required for both transcription and replication in the yeast Saccharomyces cerevisiae. Genetics, 155(4):1593-1606, 2000.

[183] M Štros, D Launholt, and K D Grasser. The HMG-box: a versatile protein domain occurring in a wide variety of DNA-binding proteins. Cellular and Molecular Life Sciences, 64(19-20):2590-2606, 2007.

[184] James E Masse, Ben Wong, Yi-Meng Yen, Frédéric H T Allain, Reid C Johnson, and Juli Feigon. The S.cerevisiae architectural HMGB protein NHP6A complexed with DNA: DNA and protein conformational changes upon Binding. Journal of Molecular Biology, 323(2):263-284, 2002.

[185] David J Stillman. Nhp6: a small but powerful effector of chromatin structure in Saccharomyces cerevisiae. Biochimica et Biophysica Acta, 1799(1-2):175-180, 2010.

[186] N K Brewster, G C Johnston, and R A Singer. A bipartite yeast SSRP1 analog comprised of Pob3 and Nhp6 proteins modulates transcription. Molecular and Cellular Biology, 21(10):3491-3502, 2001. 
[187] Alison R Rhoades, Susan Ruone, and Tim Formosa. Structural features of nucleosomes reorganized by yeast FACT and its HMG box component, Nhp6. Molecular and Cellular Biology, 24(9):3907-3917, 2004.

[188] Susan Ruone, Alison R Rhoades, and Tim Formosa. Multiple Nhp6 molecules are required to recruit Spt16-Pob3 to form yFACT complexes and to reorganize nucleosomes. Journal of Biological Chemistry, 278(46):45288-45295, 2003.

[189] Hua Xin, Shinya Takahata, Mary Blanksma, Laura McCullough, David J Stillman, and Tim Formosa. yFACT induces global accessibility of nucleosomal DNA without H2A-H2B displacement. Molecular Cell, 35(3):365-376, 2009.

[190] Seung-Woo Kang, Takashi Kuzuhara, and Masami Horikoshi. Functional interaction of general transcription initiation factor TFIIE with general chromatin factor SPT16/CDC68. Genes to Cells, 5(4):251-263, 2000.

[191] Debabrata Biswas, Rinku Dutta-Biswas, Doyel Mitra, Yoichiro Shibata, Brian D Strahl, Tim Formosa, and David J Stillman. Opposing roles for Set2 and yFACT in regulating TBP binding at promoters. The EMBO Journal, 25(19):4479-4489, 2006.

[192] Shinya Takahata, Yaxin Yu, and David J Stillman. FACT and Asf1 regulate nucleosome dynamics and coactivator binding at the HO promoter. Molecular Cell, 34(4):405-415, 2009.

[193] A Saunders. Tracking FACT and the RNA Polymerase II elongation complex through chromatin in vivo. Science, 301(5636):1094-1096, 2003.

[194] Andreas Mayer, Michael Lidschreiber, Matthias Siebert, Kristin Leike, Johannes Söding, and Patrick Cramer. Uniform transitions of the general RNA polymerase II transcription complex. Nature Structural \& Molecular Biology, 17(10):1272-1278, 2010.

[195] Karen Adelman, Wenxiang Wei, M Behfar Ardehali, Janis Werner, Bing Zhu, Danny Reinberg, and John T Lis. Drosophila Paf1 modulates chromatin structure at actively transcribed genes. Molecular and Cellular Biology, 26(1):250-260, 2006.

[196] Nevan J Krogan, Minkyu Kim, Seong Hoon Ahn, Guoqing Zhong, Michael S Kobor, Gerard Cagney, Andrew Emili, Ali Shilatifard, Stephen Buratowski, and Jack F Greenblatt. RNA polymerase II elongation factors of Saccharomyces cerevisiae: a targeted proteomics approach. Molecular and Cellular Biology, 22(20):6979-6992, 2002.

[197] Sharon L Squazzo, Patrick J Costa, Derek L Lindstrom, Kathryn E Kumer, Rajna Simic, Jennifer L Jennings, Andrew J Link, Karen M Arndt, and Grant A Hartzog. The Paf1 complex physically and functionally associates with transcription elongation factors in vivo. The EMBO Journal, 21(7):1764-1774, 2002.

[198] Rushad Pavri, Bing Zhu, Guohong Li, Patrick Trojer, Subhrangsu Mandal, Ali Shilatifard, and Danny Reinberg. Histone H2B monoubiquitination functions cooperatively with FACT to regulate elongation by RNA polymerase II. Cell, 125(4):703-717, 2006. 
[199] Wenlai Zhou, Ping Zhu, Jianxun Wang, Gabriel Pascual, Kenneth A Ohgi, Jean Lozach, Christopher K Glass, and Michael G Rosenfeld. Histone H2A monoubiquitination represses transcription by inhibiting RNA polymerase II transcriptional elongation. Molecular Cell, 29(1):69-80, 2008.

[200] So Hee Kwon, Laurence Florens, Selene K Swanson, Michael P Washburn, Susan M Abmayr, and Jerry L Workman. Heterochromatin protein 1 (HP1) connects the FACT histone chaperone complex to the phosphorylated CTD of RNA polymerase II. Genes \& Development, 24(19):2133$2145,2010$.

[201] Rajna Simic, Derek L Lindstrom, Hien G Tran, Kelli L Roinick, Patrick J Costa, Alexander D Johnson, Grant A Hartzog, and Karen M Arndt. Chromatin remodeling protein Chd1 interacts with transcription elongation factors and localizes to transcribed genes. The EMBO Journal, 22(8):1846-1856, 2003.

[202] Dawn E Kelley, David G Stokes, and Robert P Perry. CHD1 interacts with SSRP1 and depends on both its chromodomain and its ATPase/helicase-like domain for proper association with chromatin. Chromosoma, 108(1):10-25, 1999.

[203] Robert J Sims, Chi-Fu Chen, Helena Santos-Rosa, Tony Kouzarides, Smita S Patel, and Danny Reinberg. Human but not yeast CHD1 binds directly and selectively to histone H3 methylated at lysine 4 via its tandem chromodomains. Journal of Biological Chemistry, 280(51):41789-41792, 2005.

[204] Robert J Sims, Scott Millhouse, Chi-Fu Chen, Brian A Lewis, Hediye Erdjument-Bromage, Paul Tempst, James L Manley, and Danny Reinberg. Recognition of trimethylated histone H3 lysine 4 facilitates the recruitment of transcription postinitiation factors and pre-mRNA splicing. Molecular Cell, 28(4):665-676, 2007.

[205] Joanna L Birch, Bertrand C-M Tan, Kostya I Panov, Tatiana B Panova, Jens S Andersen, Tom A Owen-Hughes, Jackie Russell, Sheng-Chung Lee, and Joost C B M Zomerdijk. FACT facilitates chromatin transcription by RNA polymerases I and III. The EMBO Journal, 28(7):854-865, 2009.

[206] Vanessa Cheung, Gordon Chua, Nizar N Batada, Christian R Landry, Stephen W Michnick, Timothy R Hughes, and Fred Winston. Chromatin- and transcription-related factors repress transcription from within coding regions throughout the Saccharomyces cerevisiae genome. PLOS Biology, 6(11):e277, 2008.

[207] Catherine N Myers, Gary B Berner, Joseph H Holthoff, Kirby Martinez-Fonts, Jennifer A Harper, Sarah Alford, Megan N Taylor, and Andrea A Duina. Mutant versions of the S. cerevisiae transcription elongation factor Spt16 define regions of Spt16 that functionally interact with histone H3. PLOS ONE, 6(6):e20847, 2011.

[208] G Orphanides and D Reinberg. RNA polymerase II elongation through chromatin. Nature, 407(6803):471-475, 2000.

[209] Fu-Kai Hsieh, Olga I Kulaeva, Smita S Patel, Pamela N Dyer, Karolin Luger, Danny Reinberg, and Vasily M Studitsky. Histone chaperone FACT action during transcription through chromatin by RNA polymerase II. Proceedings of the National Academy of Sciences, 110(19):7654-7659, 2013. 
[210] Olga I Kulaeva, Daria A Gaykalova, Nikolai A Pestov, Viktor V Golovastov, Dmitry G Vassylyev, Irina Artsimovitch, and Vasily M Studitsky. Mechanism of chromatin remodeling and recovery during passage of RNA polymerase II. Nature Structural \& Molecular Biology, 16(12):1272-1278, 2009.

[211] Olga I Kulaeva and Vasily M Studitsky. Mechanism of histone survival during transcription by RNA polymerase II. Transcription, 1(2):85-88, 2010.

[212] J Wittmeyer, L Joss, and T Formosa. Spt16 and Pob3 of Saccharomyces cerevisiae form an essential, abundant heterodimer that is nuclear, chromatin-associated, and copurifies with DNA polymerase alpha. Biochemistry, 38(28):8961-8971, 1999.

[213] Yanjiao Zhou and Teresa S-F Wang. A coordinated temporal interplay of nucleosome reorganization factor, sister chromatin cohesion factor, and DNA polymerase alpha facilitates DNA replication. Molecular and Cellular Biology, 24(21):9568-9579, 2004.

[214] L Hertel, M De Andrea, G Bellomo, P Santoro, S Landolfo, and M Gariglio. The HMG protein T160 colocalizes with DNA replication foci and is down-regulated during cell differentiation. Experimental Cell Research, 250(2):313-328, 1999.

[215] Monturus Ma Estefanía, Olivier Ganier, Pablo Hernández, Jorge B Schvartzman, Marcel Mechali, and Dora B Krimer. DNA replication fading as proliferating cells advance in their commitment to terminal differentiation. Scientific Reports, 2, 2012.

[216] K Okuhara, K Ohta, H Seo, M Shioda, T Yamada, Y Tanaka, N Dohmae, Y Seyama, T Shibata, and $\mathrm{H}$ Murofushi. A DNA unwinding factor involved in DNA replication in cell-free extracts of Xenopus eggs. Current Biology, 9(7):341-350, 1999.

[217] Eva Petermann, Manuel Luís Orta, Natalia Issaeva, Niklas Schultz, and Thomas Helleday. Hydroxyurea-stalled replication forks become progressively inactivated and require two different RAD51-mediated pathways for restart and repair. Molecular Cell, 37(4):492-502, 2010.

[218] G Simchen, F Winston, C A Styles, and G R Fink. Ty-mediated gene expression of the LYS2 and HIS4 genes of Saccharomyces cerevisiae is controlled by the same SPT genes. Proceedings of the National Academy of Sciences, 81(8):2431-2434, 1984.

[219] E A Malone, C D Clark, A Chiang, and F Winston. Mutations in SPT16/CDC68 suppress cisand trans-acting mutations that affect promoter function in Saccharomyces cerevisiae. Molecular and Cellular Biology, 11(11):5710-5717, 1991.

[220] Fred Winston. Analysis of SPT Genes: A Genetic Approach toward Analysis of TFIID, Histones, and Other Transcription Factors of Yeast. Cold Spring Harbor Monograph Archive, 1992.

[221] Lena R Kundu, Masayuki Seki, Nanae Watanabe, Hiromu Murofushi, Asako Furukohri, Shou Waga, Alan J Score, J Julian Blow, Masami Horikoshi, Takemi Enomoto, and Shusuke Tada. Biphasic chromatin binding of histone chaperone FACT during eukaryotic chromatin DNA replication. Biochimica et Biophysica Acta, 1813(6):1129-1136, 2011. 
[222] Andrea Kinner, Wenqi Wu, Christian Staudt, and George Iliakis. Gamma-H2AX in recognition and signaling of DNA double-strand breaks in the context of chromatin. Nucleic Acids Research, 36(17):5678-5694, 2008.

[223] Kyu Heo, Hyunjung Kim, Si Ho Choi, Jongkyu Choi, Kyunghwan Kim, Jiafeng Gu, Michael R Lieber, Allen S Yang, and Woojin An. FACT-mediated exchange of histone variant H2AX regulated by phosphorylation of H2AX and ADP-ribosylation of Spt16. Molecular Cell, 30(1):8697, 2008.

[224] Jing-Yi Huang, Wei-Hao Chen, Ya-Ling Chang, Hsiao-Ting Wang, Wan-ting Chuang, and ShengChung Lee. Modulation of nucleosome-binding activity of FACT by poly(ADP-ribosyl)ation. Nucleic Acids Research, 34(8):2398-2407, 2006.

[225] Monica Ransom, Briana K Dennehey, and Jessica K Tyler. Chaperoning histones during DNA replication and repair. Cell, 140(2):183-195, 2010.

[226] D M Keller, X Zeng, Y Wang, Q H Zhang, M Kapoor, H Shu, R Goodman, G Lozano, Y Zhao, and $\mathrm{H} \mathrm{Lu}$. A DNA damage-induced p53 serine 392 kinase complex contains CK2, hSpt16, and SSRP1. Molecular Cell, 7(2):283-292, 2001.

[227] M Kapoor and G Lozano. Functional activation of p53 via phosphorylation following DNA damage by UV but not gamma radiation. Proceedings of the National Academy of Sciences, 95(6):28342837, 1998.

[228] N D Lakin and S P Jackson. Regulation of p53 in response to DNA damage. Oncogene, 18(53):7644-7655, 1999.

[229] Sheau-Yann Shieh, Jinwoo Ahn, Katsuyuki Tamai, Yoichi Taya, and Carol Prives. The human homologs of checkpoint kinases Chk1 and Cds1 (Chk2) phosphorylate p53 at multiple DNA damage-inducible sites. Genes \& Development, 14(3):289-300, 2000.

[230] William A Freed-Pastor and Carol Prives. Mutant p53: one name, many proteins. Genes $\mathcal{E}$ Development, 26(12):1268-1286, 2012.

[231] Yanping Li, David M Keller, John D Scott, and Hua Lu. CK2 phosphorylates SSRP1 and inhibits its DNA-binding activity. Journal of Biological Chemistry, 280(12):11869-11875, 2005.

[232] Heinz Neumann. Rewiring translation - Genetic code expansion and its applications. FEBS Letters, 586(15):2057-2064, 2012.

[233] Youngha Ryu and Peter G Schultz. Efficient incorporation of unnatural amino acids into proteins in Escherichia coli. Nature Methods, 3(4):263-265, 2006.

[234] Shawn Chen, Peter G Schultz, and Ansgar Brock. An improved system for the generation and analysis of mutant proteins containing unnatural amino acids in Saccharomyces cerevisiae. Journal of Molecular Biology, 371(1):112-122, 2007.

[235] Travis S Young, Insha Ahmad, Jun A Yin, and Peter G Schultz. An enhanced system for unnatural amino acid mutagenesis in E. coli. Journal of Molecular Biology, 395(2):361-374, 2010. 
[236] Christoph Lammers, Liljan E Hahn, and Heinz Neumann. Optimized plasmid systems for the incorporation of multiple different unnatural amino acids by evolved orthogonal ribosomes. ChemBioChem, 15(12):1800-1804, 2014.

[237] M H Richmond. The effect of amino acid analogues on growth and protein synthesis in microorganisms. Bacteriological Reviews, 26(4):398-420, 1962.

[238] D B Cowie and G N Cohen. Biosynthesis by Escherichia coli of active altered proteins containing selenium instead of sulfur. Biochimica et Biophysica Acta, 26(2):252-261, 1957.

[239] A Böck, K Forchhammer, J Heider, W Leinfelder, G Sawers, B Veprek, and F Zinoni. Selenocysteine: the 21st amino acid. Molecular Microbiology, 5(3):515-520, 1991.

[240] Gayathri Srinivasan, Carey M James, and Joseph A Krzycki. Pyrrolysine encoded by UAG in Archaea: charging of a UAG-decoding specialized tRNA. Science, 296(5572):1459-1462, 2002.

[241] R Furter. Expansion of the genetic code: site-directed p-fluoro-phenylalanine incorporation in Escherichia coli. Protein Science, 7(2):419-426, 1998.

[242] L Wang, A Brock, B Herberich, and P G Schultz. Expanding the genetic code of Escherichia coli. Science, 292(5516):498-500, 2001.

[243] Jason W Chin. Expanding and reprogramming the genetic code of cells and animals. Annual Review of Biochemistry, 83:379-408, 2014.

[244] Heinz Neumann, Sew Y Peak-Chew, and Jason W Chin. Genetically encoding N I [epsi] I acetyllysine in recombinant proteins. Nature Chemical Biology, 4(4):232-234, 2008.

[245] Duy P Nguyen, Maria M Garcia Alai, Prashant B Kapadnis, Heinz Neumann, and Jason W Chin. Genetically encoding N(epsilon)-methyl-L-lysine in recombinant histones. Journal of the American Chemical Society, 131(40):14194-14195, 2009.

[246] Duy P Nguyen, Maria M Garcia Alai, Satpal Virdee, and Jason W Chin. Genetically directing e-N, N-dimethyl-L-lysine in recombinant histones. Chemistry \& Biology, 17(10):1072-1076, 2010.

[247] Tilman Plass, Sigrid Milles, Christine Koehler, Carsten Schultz, and Edward A Lemke. Genetically encoded copper-free click chemistry. Angewandte Chemie, 50(17):3878-3881, 2011.

[248] Kathrin Lang, Lloyd Davis, Stephen Wallace, Mohan Mahesh, Daniel J Cox, Melissa L Blackman, Joseph M Fox, and Jason W Chin. Genetic Encoding of bicyclononynes and trans-cyclooctenes for site-specific protein labeling in vitro and in live mammalian cells via rapid fluorogenic Diels-Alder reactions. Journal of the American Chemical Society, 134(25):10317-10320, 2012.

[249] Kathrin Lang, Lloyd Davis, Jessica Torres-Kolbus, Chungjung Chou, Alexander Deiters, and Jason W Chin. Genetically encoded norbornene directs site-specific cellular protein labelling via a rapid bioorthogonal reaction. Nature Chemistry, 4(4):298-304, 2012.

[250] James Hemphill, Chungjung Chou, Jason W Chin, and Alexander Deiters. Genetically encoded light-activated transcription for spatiotemporal control of gene expression and gene silencing in mammalian cells. Journal of the American Chemical Society, 135(36):13433-13439, 2013. 
[251] James Hemphill, Jeane Govan, Rajendra Uprety, Michael Tsang, and Alexander Deiters. Sitespecific promoter caging enables optochemical gene activation in cells and animals. Journal of the American Chemical Society, 136(19):7152-7158, 2014.

[252] Steven Walsh, Laura Gardner, Alexander Deiters, and Gavin J Williams. Intracellular lightactivation of riboswitch activity. ChemBioChem, 15(9):1346-1351, 2014.

[253] Yoshihito Tanaka, Michelle R Bond, and Jennifer J Kohler. Photocrosslinkers illuminate interactions in living cells. Molecular BioSystems, 4(6):473-480, 2008.

[254] G Dormán and G D Prestwich. Benzophenone photophores in biochemistry. Biochemistry, 33(19):5661-5673, 1994.

[255] Jason W Chin, T Ashton Cropp, J Christopher Anderson, Mridul Mukherji, Zhiwen Zhang, and Peter G Schultz. An expanded eukaryotic genetic code. Science, 301(5635):964-967, 2003.

[256] Angela Wittelsberger, Beena E Thomas, Dale F Mierke, and Michael Rosenblatt. Methionine acts as a "magnet" in photoaffinity crosslinking experiments. FEBS Letters, 580(7):1872-1876, 2006.

[257] K A Schnapp, R Poe, E Leyva, N Soundararajan, and M S Platz. Exploratory photochemistry of fluorinated aryl azides. Implications for the design of photoaffinity labeling reagents. Bioconjugate Chemistry, 4(2):172-177, 1993.

[258] Jeremy R Knowles. Photogenerated reagents for biological receptor-site labeling. Accounts of Chemical Research, 5(4):155-160, 1972.

[259] James V Staros, Hagan Bayley, David N Standring, and Jeremy R Knowles. Reduction of aryl azides by thiols: Implications for the use of photoaffinity reagents. Biochemical and Biophysical Research Communications, 80(3):568-572, 1978.

[260] Jason W Chin, Andrew B Martin, David S King, Lei Wang, and Peter G Schultz. Addition of a photocrosslinking amino acid to the genetic code of Escherichiacoli. Proceedings of the National Academy of Sciences, 99(17):11020-11024, 2002.

[261] Jason W Chin, Stephen W Santoro, Andrew B Martin, David S King, Lei Wang, and Peter G Schultz. Addition of p-azido-L-phenylalanine to the genetic code of Escherichia coli. Journal of the American Chemical Society, 124(31):9026-9027, 2002.

[262] Hiroyuki Mori and Koreaki Ito. Different modes of SecY-SecA interactions revealed by sitedirected in vivo photo-cross-linking. Proceedings of the National Academy of Sciences, 103(44):1615916164, 2006.

[263] Sanchaita Das and Donald B Oliver. Mapping of the SecA·SecY and SecA·SecG interfaces by site-directed in vivo photocross-linking. Journal of Biological Chemistry, 286(14):12371-12380, 2011.

[264] Christian Schlieker, Jimena Weibezahn, Holger Patzelt, Peter Tessarz, Christine Strub, Kornelius Zeth, Annette Erbse, Jens Schneider-Mergener, Jason W Chin, Peter G Schultz, Bernd Bukau, and Axel Mogk. Substrate recognition by the AAA+ chaperone ClpB. Nature Structural \& Molecular Biology, 11(7):607-615, 2004. 
[265] Frieder Merz, Daniel Boehringer, Christiane Schaffitzel, Steffen Preissler, Anja Hoffmann, Timm Maier, Anna Rutkowska, Jasmin Lozza, Nenad Ban, Bernd Bukau, and Elke Deuerling. Molecular mechanism and structure of Trigger Factor bound to the translating ribosome. The EMBO Journal, 27(11):1622-1632, 2008.

[266] Hyun Soo Lee, Romerson D Dimla, and Peter G Schultz. Protein-DNA photo-crosslinking with a genetically encoded benzophenone-containing amino acid. Bioorganic $\mathcal{E}$ Medicinal Chemistry Letters, 19(17):5222-5224, 2009.

[267] H M Goodman, J Abelson, A Landy, S Brenner, and J D Smith. Amber suppression: a nucleotide change in the anticodon of a tyrosine transfer RNA. Nature, 217(5133):1019-1024, 1968.

[268] Pedro Carvalho, Ann Marie Stanley, and Tom A Rapoport. Retrotranslocation of a misfolded luminal ER protein by the ubiquitin-ligase Hrd1p. Cell, 143(4):579-591, 2010.

[269] Ann Marie Stanley, Pedro Carvalho, and Tom Rapoport. Recognition of an ERAD-L substrate analyzed by site-specific in vivo photocrosslinking. FEBS Letters, 585(9):1281-1286, 2011.

[270] Yasushi Tamura, Yoshihiro Harada, Takuya Shiota, Koji Yamano, Kazuaki Watanabe, Mihoko Yokota, Hayashi Yamamoto, Hiromi Sesaki, and Toshiya Endo. Tim23-Tim50 pair coordinates functions of translocators and motor proteins in mitochondrial protein import. The Journal of Cell Biology, 184(1):129-141, 2009.

[271] Christian Schulz, Oleksandr Lytovchenko, Jonathan Melin, Agnieszka Chacinska, Bernard Guiard, Piotr Neumann, Ralf Ficner, Olaf Jahn, Bernhard Schmidt, and Peter Rehling. Tim50's presequence receptor domain is essential for signal driven transport across the TIM23 complex. The Journal of Cell Biology, 195(4):643-656, 2011.

[272] Koji Yamano, Sachiko Tanaka-Yamano, and Toshiya Endo. Tom7 regulates Mdm10-mediated assembly of the mitochondrial import channel protein Tom40. Journal of Biological Chemistry, 285(53):41222-41231, 2010.

[273] Neeman Mohibullah and Steven Hahn. Site-specific cross-linking of TBP in vivo and in vitro reveals a direct functional interaction with the SAGA subunit Spt3. Genes E Development, 22(21):2994-3006, 2008.

[274] Nobumasa Hino, Yuko Okazaki, Takatsugu Kobayashi, Akiko Hayashi, Kensaku Sakamoto, and Shigeyuki Yokoyama. Protein photo-cross-linking in mammalian cells by site-specific incorporation of a photoreactive amino acid. Nature Methods, 2(3):201-206, 2005.

[275] Kensaku Sakamoto, Akiko Hayashi, Ayako Sakamoto, Daisuke Kiga, Hiroshi Nakayama, Akiko Soma, Takatsugu Kobayashi, Makoto Kitabatake, Koji Takio, Kazuki Saito, Mikako Shirouzu, Ichiro Hirao, and Shigeyuki Yokoyama. Site-specific incorporation of an unnatural amino acid into proteins in mammalian cells. Nucleic Acids Research, 30(21):4692-4699, 2002.

[276] Nobumasa Hino, Masaaki Oyama, Aya Sato, Takahito Mukai, Fumie Iraha, Akiko Hayashi, Hiroko Kozuka-Hata, Tadashi Yamamoto, Shigeyuki Yokoyama, and Kensaku Sakamoto. Genetic incorporation of a photo-crosslinkable amino acid reveals novel protein complexes with GRB2 in mammalian cells. Journal of Molecular Biology, 406(2):343-353, 2011. 
[277] Annette G Beck Sickinger and Nediljko Budisa. Genetically encoded photocrosslinkers as molecular probes to study G-Protein-Coupled Receptors (GPCRs). Angewwandte Chemie, 51(2):310$312,2012$.

[278] Kelly A Daggett and Thomas P Sakmar. Site-specific in vitro and in vivo incorporation of molecular probes to study G-protein-coupled receptors. Current Opinion in Chemical Biology, 15(3):392-398, 2011.

[279] Won-Ki Huh, James V Falvo, Luke C Gerke, Adam S Carroll, Russell W Howson, Jonathan S Weissman, and Erin K O'Shea. Global analysis of protein localization in budding yeast. Nature, 425(6959):686-691, 2003.

[280] Sina Ghaemmaghami, Won-Ki Huh, Kiowa Bower, Russell W Howson, Archana Belle, Noah Dephoure, Erin K O'Shea, and Jonathan S Weissman. Global analysis of protein expression in yeast. Nature Cell Biology, 425(6959):737-741, 2003.

[281] Zhijian Li, Franco J Vizeacoumar, Sondra Bahr, Jingjing Li, Jonas Warringer, Frederick S Vizeacoumar, Renqiang Min, Benjamin VanderSluis, Jeremy Bellay, Michael DeVit, James A Fleming, Andrew Stephens, Julian Haase, Zhen-Yuan Lin, Anastasia Baryshnikova, Hong Lu, Zhun Yan, Ke Jin, Sarah Barker, Alessandro Datti, Guri Giaever, Corey Nislow, Chris Bulawa, Chad L Myers, Michael Costanzo, Anne-Claude Gingras, Zhaolei Zhang, Anders Blomberg, Kerry Bloom, Brenda Andrews, and Charles Boone. Systematic exploration of essential yeast gene function with temperature-sensitive mutants. Nature Biotechnology, 29(4):361-367, 2011.

[282] Yifat Cohen and Maya Schuldiner. Advanced methods for high-throughput microscopy screening of genetically modified yeast libraries. Methods in Molecular Biology, 781:127-159, 2011.

[283] Caroline A Schneider, Wayne S Rasband, and Kevin W Eliceiri. NIH Image to ImageJ: 25 years of image analysis. Nature Methods, 9(7):671-675, 2012.

[284] Johannes Schindelin, Ignacio Arganda-Carreras, Erwin Frise, Verena Kaynig, Mark Longair, Tobias Pietzsch, Stephan Preibisch, Curtis Rueden, Stephan Saalfeld, Benjamin Schmid, JeanYves Tinevez, Daniel James White, Volker Hartenstein, Kevin Eliceiri, Pavel Tomancak, and Albert Cardona. Fiji: an open-source platform for biological-image analysis. Nature Methods, 9(7):676-682, 2012.

[285] U Güldener and J H Hegemann. A second generation of GFP-vectors for subcellular localization studies in budding yeast. Technical report, 2013.

[286] F Sanger, S Nicklen, and A R Coulson. DNA sequencing with chain-terminating inhibitors. Proceedings of the National Academy of Sciences, 74(12):5463-5467, 1977.

[287] Cornel Mülhardt. Der Experimentator: Molekularbiologie / Genomics. Springer-Verlag, 2009.

[288] Thomas W Christianson, Robert S Sikorski, Michael Dante, James H Shero, and Philip Hieter. Multifunctional yeast high-copy-number shuttle vectors. Gene, 110(1):119-122, 1992.

[289] D Mumberg, R Müller, and M Funk. Regulatable promoters of Saccharomyces cerevisiae: comparison of transcriptional activity and their use for heterologous expression. Nucleic Acids Research, 22(25):5767-5768, 1994. 
[290] M Knop, K Siegers, G Pereira, W Zachariae, B Winsor, K Nasmyth, and E Schiebel. Epitope tagging of yeast genes using a PCR-based strategy: more tags and improved practical routines. Yeast, 15(10B):963-972, 1999.

[291] M P Yaffe and G Schatz. Two nuclear mutations that block mitochondrial protein import in yeast. Proceedings of the National Academy of Sciences, 81(15):4819-4823, 1984.

[292] U K Laemmli. Cleavage of structural proteins during the assembly of the head of bacteriophage T4. Nature, 227(5259):680-685, 1970.

[293] P T Lowary and J Widom. New DNA sequence rules for high affinity binding to histone octamer and sequence-directed nucleosome positioning. Journal of Molecular Biology, 276(1):19-42, 1998.

[294] A Thåström, P T Lowary, H R Widlund, H Cao, M Kubista, and J Widom. Sequence motifs and free energies of selected natural and non-natural nucleosome positioning DNA sequences. Journal of Molecular Biology, 288(2):213-229, 1999.

[295] R Daniel Gietz and Robert H Schiestl. Frozen competent yeast cells that can be transformed with high efficiency using the LiAc/SS carrier DNA/PEG method. Nature Protocols, 2(1):1-4, 2007.

[296] F Sherman. An introduction to the genetics and molecular biology of the yeast Saccharomyces cerevisiae. The Encyclopedia of Molecular Biology and Molecular Medicine, 6:302-325, 1998.

[297] Ian S Farrell, Rebecca Toroney, Jennifer L Hazen, Ryan A Mehl, and Jason W Chin. Photocross-linking interacting proteins with a genetically encoded benzophenone. Nature Methods, 2(5):377-384, 2005.

[298] Shunichi Kosugi, Masako Hasebe, Masaru Tomita, and Hiroshi Yanagawa. Systematic identification of cell cycle-dependent yeast nucleocytoplasmic shuttling proteins by prediction of composite motifs. Proceedings of the National Academy of Sciences, 106(25):10171-10176, 2009.

[299] Alex N Nguyen Ba, Anastassia Pogoutse, Nicholas Provart, and Alan M Moses. NLStradamus: a simple Hidden Markov Model for nuclear localization signal prediction. BMC Bioinformatics, 10(1):202, 2009.

[300] Allison Lange, Ryan E Mills, Christopher J Lange, Murray Stewart, Scott E Devine, and Anita H Corbett. Classical nuclear localization signals: definition, function, and interaction with importin alpha. Journal of Biological Chemistry, 282(8):5101-5105, 2007.

[301] D T Beranek. Distribution of methyl and ethyl adducts following alkylation with monofunctional alkylating agents. Mutation Research, 231(1):11-30, 1990.

[302] Cecilia Lundin, Matthew North, Klaus Erixon, Kevin Walters, Dag Jenssen, Alastair S H Goldman, and Thomas Helleday. Methyl methanesulfonate (MMS) produces heat-labile DNA damage but no detectable in vivo DNA double-strand breaks. Nucleic Acids Research, 33(12):3799$3811,2005$.

[303] Sebastian Nehring, Nediljko Budisa, and Birgit Wiltschi. Performance analysis of orthogonal pairs designed for an expanded eukaryotic genetic code. PLOS ONE, 7(4):e31992, 2012. 
[304] Douglas Maya Mari Cruz Muñoz-Centeno Rakesh Kumar Singh Vincent Oreal Gajjalaiahvari Ugander Reddy Dun Liang Vincent Géli Akash Gunjan Sebastián Chávez Macarena MorilloHuesca. FACT prevents the accumulation of free histones evicted from transcribed chromatin and a subsequent cell cycle delay in G1. PLOS Genetics, 6(5), 2010.

[305] Katsunori Yoshikawa, Tadamasa Tanaka, Yoshihiro Ida, Chikara Furusawa, Takashi Hirasawa, and Hiroshi Shimizu. Comprehensive phenotypic analysis of single-gene deletion and overexpression strains of Saccharomyces cerevisiae. Yeast, 28(5):349-361, 2011.

[306] Y Nakamura, M Uno, T Toyoda, T Fujiwara, and K Ito. Protein tRNA mimicry in translation termination. Cold Spring Harbor Symposia on Quantitative Biology, 66(0):469-476, 2001.

[307] Jason W Chin, T Ashton Cropp, Stephanie Chu, Eric Meggers, and Peter G Schultz. Progress toward an expanded eukaryotic genetic code. Chemistry \& Biology, 10(6):511-519, 2003.

[308] Yao-Fu Chang, J Saadi Imam, and Miles F Wilkinson. The Nonsense-Mediated Decay RNA surveillance pathway. Annual Review of Biochemistry, 76(1):51-74, 2007.

[309] Sebastian Greiss and Jason W Chin. Expanding the genetic code of an animal. Journal of the American Chemical Society, 133(36):14196-14199, 2011.

[310] Qian Wang and Lei Wang. New methods enabling efficient incorporation of unnatural amino acids in yeast. Journal of the American Chemical Society, 130(19):6066-6067, 2008.

[311] Michael Berg, Annette Michalowski, Silke Palzer, Steffen Rupp, and Kai Sohn. An in vivo photo-cross-linking approach reveals a homodimerization domain of Aha1 in S. cerevisiae. PLOS ONE, 9(3):e89436, 2014.

[312] W G Nelson and M B Kastan. DNA strand breaks: the DNA template alterations that trigger p53dependent DNA damage response pathways. Molecular and Cellular Biology, 14(3):1815-1823, 1994.

[313] S Kozmin, G Slezak, A Reynaud-Angelin, C Elie, Y de Rycke, S Boiteux, and E Sage. UVA radiation is highly mutagenic in cells that are unable to repair 7,8-dihydro-8-oxoguanine in Saccharomyces cerevisiae. Proceedings of the National Academy of Sciences, 102(38):13538-13543, 2005.

[314] J D Boeke, J Trueheart, G Natsoulis, and G R Fink. 5-Fluoroorotic acid as a selective agent in yeast molecular genetics. Methods in Enzymology, 154:164-175, 1987.

[315] Briana K Dennehey, Seth Noone, Wallace H Liu, Luke Smith, Mair E A Churchill, and Jessica K Tyler. The $\mathrm{C}$ terminus of the histone chaperone Asf1 cross-links to histone $\mathrm{H} 3$ in yeast and promotes interaction with histones H3 and H4. Molecular and Cellular Biology, 33(3):605-621, 2013.

[316] Oleksandr Lytovchenko, Jonathan Melin, Christian Schulz, Markus Kilisch, Dana P Hutu, and Peter Rehling. Signal recognition initiates reorganization of the presequence translocase during protein import. The EMBO Journal, 32(6):886-898, 2013. 
[317] Isbaal Ramos, Jaime Martín-Benito, Ron Finn, Laura Bretaña, Kerman Aloria, Jesús M Arizmendi, Juan Ausió, Arturo Muga, José M Valpuesta, and Adelina Prado. Nucleoplasmin binds histone H2A-H2B dimers through its distal face. Journal of Biological Chemistry, 285(44):3377133778, 2010.

[318] Jody K Lancia, Adaora Nwokoye, Amanda Dugan, Cassandra Joiner, Rachel Pricer, and Anna K Mapp. Sequence context and crosslinking mechanism affect the efficiency of in vivo capture of a protein-protein interaction. Biopolymers, 101(4):391-397, 2014.

[319] Stefan Amlacher, Phillip Sarges, Dirk Flemming, Vera van Noort, Ruth Kunze, Damien P Devos, Manimozhiyan Arumugam, Peer Bork, and Ed Hurt. Insight into structure and assembly of the nuclear pore complex by utilizing the genome of a eukaryotic thermophile. Cell, 146(2):277-289, 2011.

[320] Kristin M Keck and Lucy F Pemberton. Histone chaperones link histone nuclear import and chromatin assembly. BBA - Gene Regulatory Mechanisms, 1819(3-4):277-289, 2012.

[321] Mary Miyaji-Yamaguchi, Kohsuke Kato, Ryosuke Nakano, Tomohiro Akashi, Akihiko Kikuchi, and Kyosuke Nagata. Involvement of nucleocytoplasmic shuttling of yeast Nap1 in mitotic progression. Molecular and Cellular Biology, 23(18):6672-6684, 2003.

[322] Young-Jun Park, Steven J McBryant, and Karolin Luger. A beta-hairpin comprising the nuclear localization sequence sustains the self-associated states of nucleosome assembly protein 1 . Journal of Molecular Biology, 375(4):1076-1085, 2008.

[323] Joseph Schacherer, Jacky de Montigny, Anne Welcker, Jean-Luc Souciet, and Serge Potier. Duplication processes in Saccharomyces cerevisiae haploid strains. Nucleic Acids Research, 33(19):6319-6326, 2005.

[324] Joseph Schacherer, Yves Tourrette, Serge Potier, Jean-Luc Souciet, and Jacky de Montigny. Spontaneous duplications in diploid Saccharomyces cerevisiae cells. DNA Repair, 6(10):14411452, 2007.

[325] Bryan J Wilkins, Kelly A Daggett, and T Ashton Cropp. Peptide mass fingerprinting using isotopically encoded photo-crosslinking amino acids. Molecular BioSystems, 4(9):934-936, 2008.

[326] Susan M Hancock, Rajendra Uprety, Alexander Deiters, and Jason W Chin. Expanding the genetic code of yeast for incorporation of diverse unnatural amino acids via a pyrrolysyl-tRNA synthetase/tRNA pair. Journal of the American Chemical Society, 132(42):14819-14824, 2010.

[327] Edward A Lemke, Daniel Summerer, Bernhard H Geierstanger, Scott M Brittain, and Peter G Schultz. Control of protein phosphorylation with a genetically encoded photocaged amino acid. Nature Chemical Biology, 3(12):769-772, 2007.

[328] A H Tong, M Evangelista, A B Parsons, H Xu, G D Bader, N Pagé, M Robinson, S Raghibizadeh, C W Hogue, H Bussey, B Andrews, M Tyers, and C Boone. Systematic genetic analysis with ordered arrays of yeast deletion mutants. Science, 294(5550):2364-2368, 2001. 


\section{Acknowledgements}

My profound gratitude goes to my supervisor, Prof. Heinz Neumann, to whom I am thankful for all these years of advice and support. I am grateful for his continuous trust being an inspiring teacher.

I want to thank, Dr. Wolfgang Fischle and Prof. Steven Johnsen, for their advice and feedback as members of my thesis committee. The fruitful discussions contributed considerably to my thesis. Furthermore, I want to thank Prof. Detlef Doenecke, Dr. Hans Dieter Schmitt and Prof. Holger Stark for accepting to take part in evaluating this thesis.

I am grateful to the staff of the Molecular Structural Biology department of Prof. Ralf Ficner. The possibility to work in a well-functioning surrounding is a great pleasure. Special thanks go to Marita Kalck for her administrative support. I would also like to thank Kerstin and Steffen of the IMPRS Molecular Biology office for all time support and advice.

I am indebted to Dr. Bryan Wilkins rising my interest for the crosslinking technique and sharing the excitement on science. Many thanks for the critical reading of this thesis and for introducing me to the American culture besides giving away the secret of baseball.

I would like to acknowledge all my lab mates for the wonderful time both in and out of the lab. Thank you for the refreshing working environment and your patience. Special thanks to Dr. Christoph Lammers for numerous hours of discussion and constant encouragement if things were not working out. For technical support, I want to thank Annette Geisler and Marco Winkler providing me always a helping hand. 
Further acknowledgements go to all my colleagues and friends in Göttingen with whom I shared passion for long-distance cycling and photography. In all aspects, this time contributed vastly to my personality. Special thanks go to my long-lasting friends Sven and Thomas for their constant support and patience during glory and hard times.

In immeasurable gratefulness and love, I want to thank my partner for being at my side and making life worth living.

Finally, I want to express my deep gratitude to my parents, Heike and Dietmar, for their support and encouragement beyond all description. I want to stress out their thoughtful advices and chaperoning guidance. A special place will always be reserved for my sister, Rita, because of love and care for her younger brother. 



\section{Appendix}

Table 5.1: General cloning and sequencing primers

\begin{tabular}{|c|c|c|}
\hline Primer number & Primer name (full) & Sequence \\
\hline \#CHR0048 & \#CHR0048 yPob3-Xhol-rv & TGATCTCGAGCTATTCTACCTTAGGCTTCTTCG \\
\hline \#CHR0062 & \#CHR0062 ynhp6-XhoI-r & TGATCTCGAGCTAAGCCAAAGTGGCGTTATATAAC \\
\hline \multirow[t]{2}{*}{ \#CHR0063 } & \#CHR0063 BamHI-TEV-ynhp6a-f & ATCAGGATCCGGAAAACCTGTATTTTCAGGGCG \\
\hline & & TCACCCCAAGAGAACCTAAG \\
\hline \#CHR0068 & \#CHR0068 seqPob3 & TTGACCTCTAAAAATGAAGTAG \\
\hline \#CHR0144 & \#CHR0144 Sall 3HA fw & GCAGGTCGACGGATCCGGAATCTTTTACC \\
\hline \#CHR0145 & \#CHR0145 3HA XhoI rv & CGCTCTCGAGGAAGTGGCGCGCCTTAGCACTG \\
\hline \#CHR0146 & \#CHR0146 SalI 3Myc1 fw & 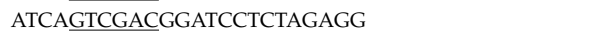 \\
\hline \#CHR0147 & \#CHR0147 3Myc1 Xhol rv & ATCACTCGAGCGCGCCTTAGACTCTAGATGATCCGTTCAAG \\
\hline \#CHR0148 & \#CHR0148 SalI 6HA fw & ATCAGTCGACTCCGGTTCTGCTGC \\
\hline \#CHR0149 & \#CHR0149 6HA XhoI rv & ATCACTCGAGTGATCGTTCCACTTTTTAGC \\
\hline \#CHR0150 & \#CHR0150 SacI Spt16 fw & 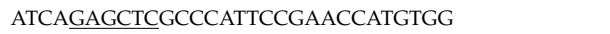 \\
\hline \#CHR0151 & \#CHR0151 Spt16 SmaI rv & ATCACCCGGGATCTCTAAAGTTTGCACCCC \\
\hline \#CHR0153 & \#CHR0153 Pob3 SalI rv & 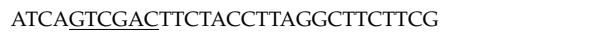 \\
\hline \#CHR0154 & \#CHR0154 SpeI Pob3 fw & 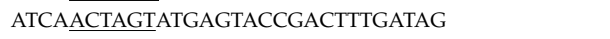 \\
\hline \#CHR0204 & \#CHR0204 CHTH Pob3-1-r & GGCGAGCCGCTCGTAGTCCTCTTGTTGGAAGCCATC \\
\hline \#CHR0205 & \#CHR0205 CHTH Pob3-2-f & GATGGCTTCCAACAAGAGGACTACGAGCGGCTCGCC \\
\hline \#CHR0206 & \#CHR0206 CHTH Pob3-2-r & TCGATATCGAACCGACCTCTTGGTGTGAGATGCAAG \\
\hline \#CHR0207 & \#CHR0207 CHTH Pob3-3-f & СTTGCATCTCACACCAAGAGGTCGGTTCGATATCGA \\
\hline \#CHR0208 & \#CHR0208 CHTH Pob3-3-r & CCGTAGTGGCCACGGTGGGTTGTGAAATCCTTGGCC \\
\hline \#CHR0209 & \#CHR0209 CHTH Pob3-4-f & GGCCAAGGATTTCACAACCCACCGTGGCCACTACGG \\
\hline \#CHR0223 & \#CHR0223 CHTH gPob3 EcoRI f & ATCAGAATTCGATGGCTGCTATGTGAGTCCT \\
\hline \#CHR0224 & \#CHR0224 CHTH gPob3 HindIII r & 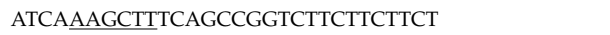 \\
\hline \#CHR0388 & \#CHR0388 QC1 f & GAATTCGATGGCTGCTATTGAGAGCTTCGACCACAT \\
\hline \#CHR0389 & \#CHR0389 QC1 r & ATGTGGTCGAAGCTCTCAATAGCAGCCATCGAATTC \\
\hline \#CHR0390 & \#CHR0390 QC2-TEV f & GCCAGGATCCGAATTCGGAAAACCTGTATTTTCAGGG \\
\hline & & CATGGCTGCTATTGAGAGCTTCGACCACAT \\
\hline \#CHR0391 & \#CHR0391 QC2-TEV r & ATGTGGTCGAAGCTCTCAATAGCAGCCATGCCCTGAA \\
\hline & & AATACAGGTTTTCCGAATTCGGATCCTGGC \\
\hline \#CHR0607 & \#CHR0607 d544-552 fw & CGACGAAGAAGTCGACCTCGACATGTCTAAAGGTG \\
\hline \#CHR0608 & \#CHR0608 d544-552 rv & CGAGGTCGACTTCTTCGTCGCTACCССССTCAGC \\
\hline \#CHR0609 & \#CHR0609 K547M fw & AAGGCCTTCGATGAAGCCTAAGGTAGAAGTCGAC \\
\hline \#CHR0610 & \#CHR0610 K547M rv & CCTTAGGCTTCATCGAAGGCCTTTCTTCGTCGC \\
\hline \#CHR0613 & \#CHR0613 d458-552 fw & TAAGGAAGAGGTCGACCTCGACATGTCTAAAGGTG \\
\hline \#CHR0614 & \#CHR0614 d458-552 rv & CGAGGTCGACCTCTTCCTTACTGATGTTGGCAAAAG \\
\hline \#CHR0617 & \#CHR0617 d458-543 fw & TAAGGAAGAGAGGCCTTCGAAGAAGCCTAAGGTAG \\
\hline \#CHR0618 & \#CHR0618 d458-543 rv & TCGAAGGCCTCTCTTCCTTACTGATGTTGGCAAAAG \\
\hline \#CHR0625 & \#CHR0625 ctPob3 dD467-571 fw & GATCGACGAGTGAAAGCTTGCGGCCGCATAATGC \\
\hline \#CHR0626 & \#CHR0626 ctPob3 dD467-571 rv & CAAGCTTTCACTCGTCGATCTCATTCTTAACCTTC \\
\hline \#CHR0627 & \#CHR0627 yPob3 BamHI rv & TGATGGATCCCTATTCTACCTTAGGCTTCTTCG \\
\hline \#CHR0628 & \#CHR0628 SGA BamHI fw & ATCAGGATCCCCTTTACGTCAAGGACAGACCACC \\
\hline \#CHR0629 & \#CHR0629 SGA Xhol fw & ATCACTCGAGCCTTTACGTCAAGGACAGACCACC \\
\hline \#CHR0632 & \#CHR0632 ctPob3 gPROVE fw3 & ATCAGGATCCTTGTTTCGTTACCAAAGGCAG \\
\hline \#CHR0875 & \#CHR0875 QC d501-NLS fw & TATGGGTTCCAGGCCTTCGAAGAAGCCTAAGG \\
\hline \#CHR0876 & \#CHR0876 QC d501-NLS rv & TCGAAGGCCTGGAACCCATATTAATATCCTC \\
\hline \#CHR0877 & \#CHR0877 QC d491-NLS fw & AGGTTCAGACAGGCCTTCGAAGAAGCCTAAGG \\
\hline \#CHR0878 & \#CHR0878 QC d491-NLS rv & TTCGAAGGCCTGTCTGAACCTAAAGCGGTTTG \\
\hline
\end{tabular}




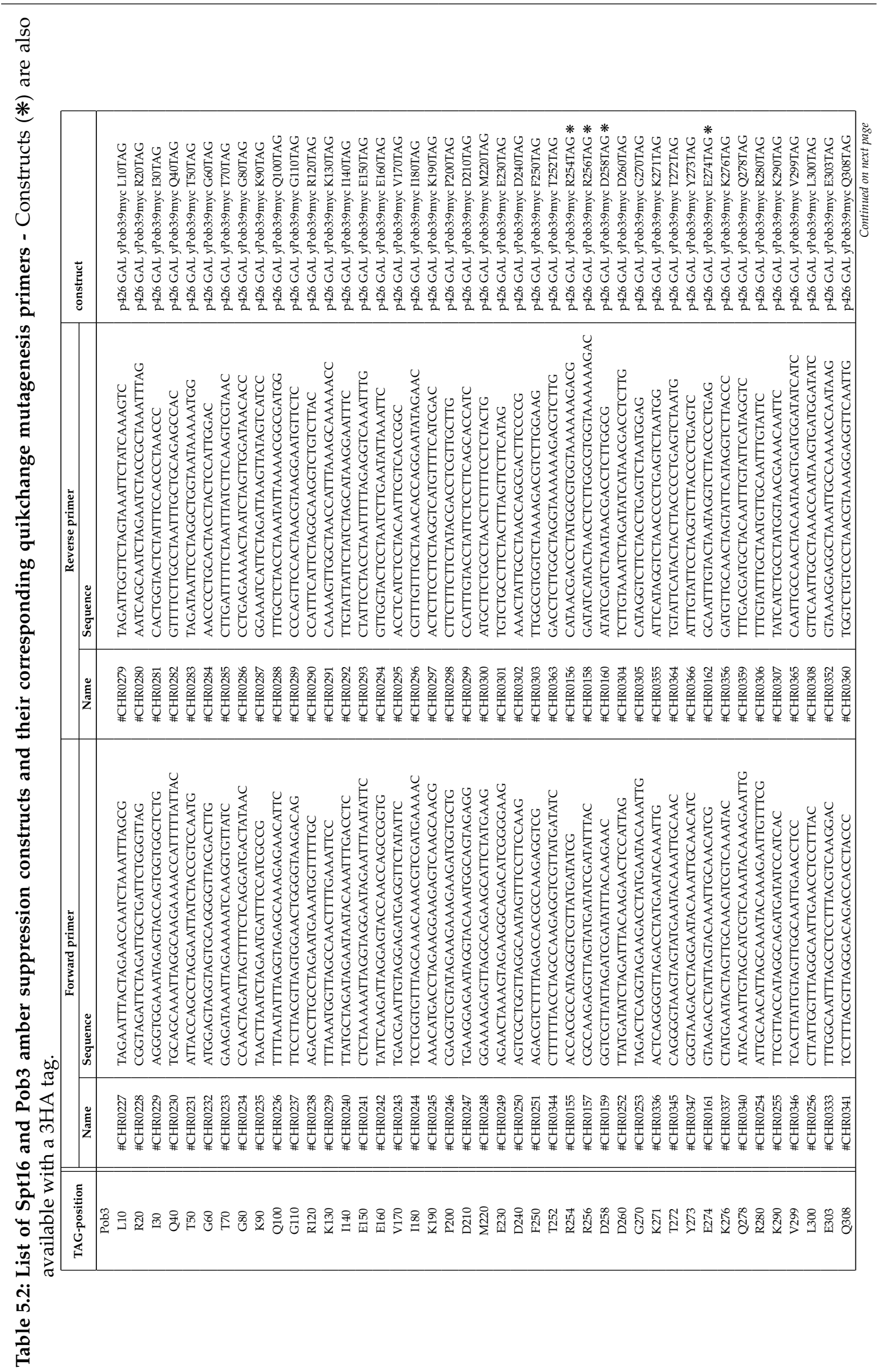




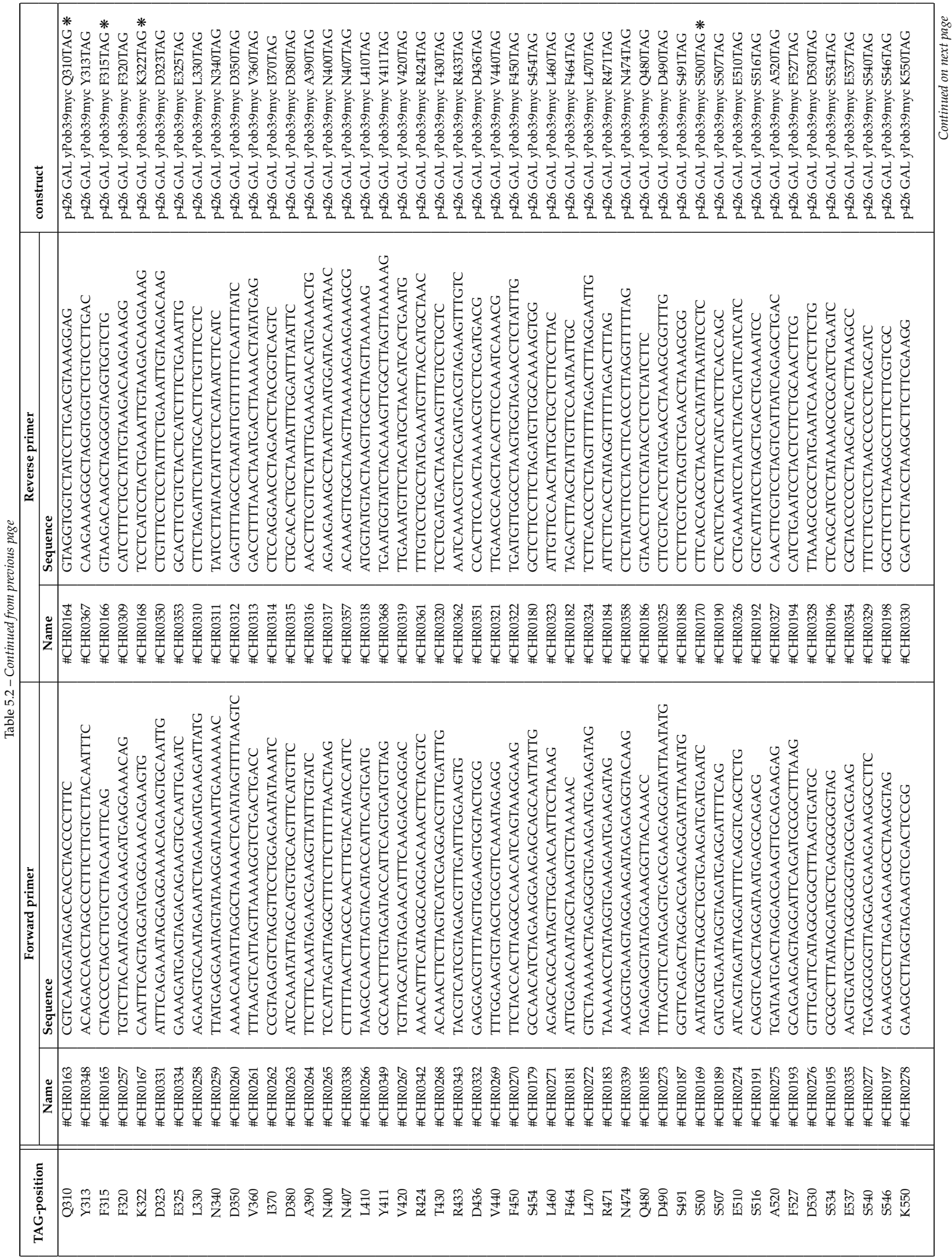




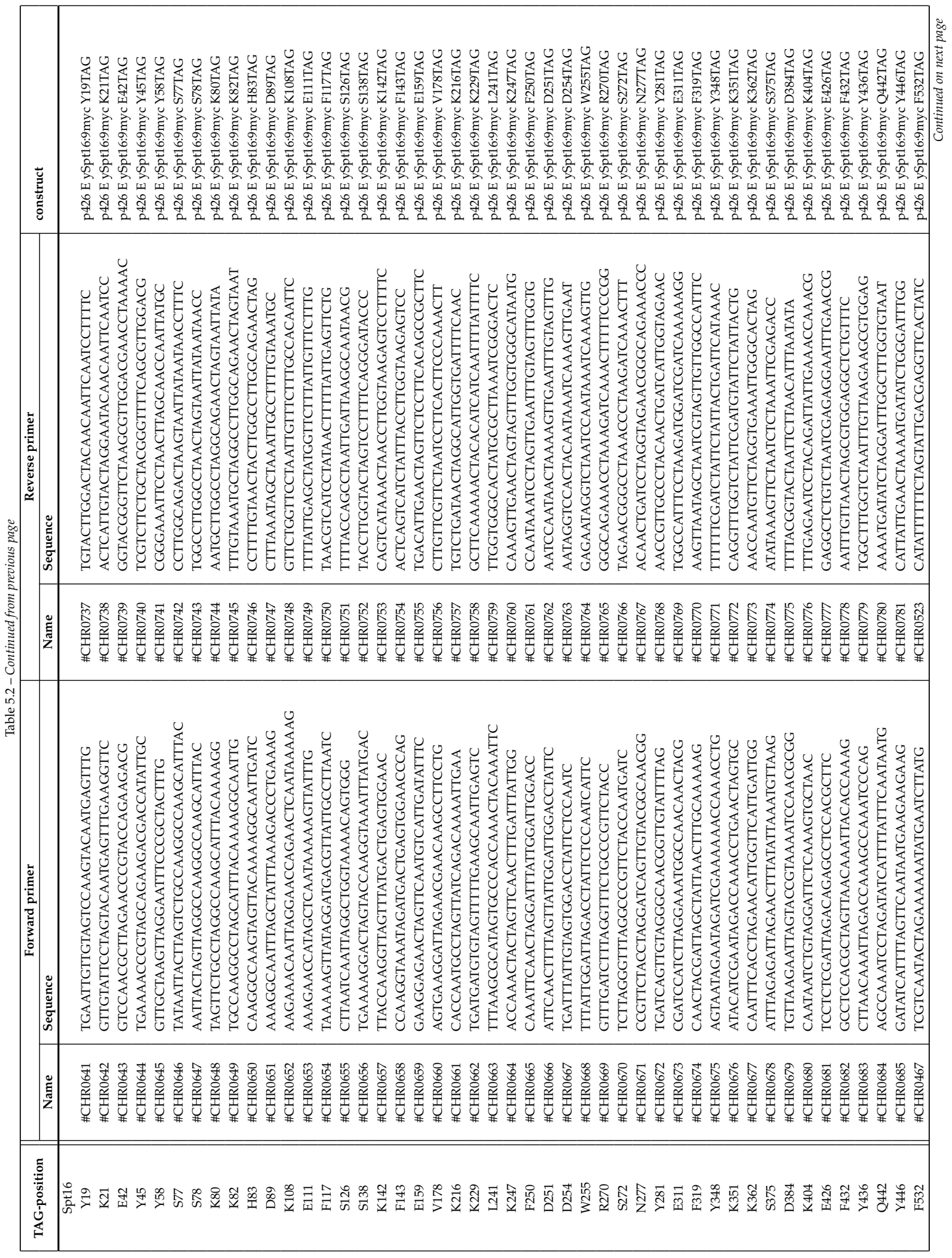




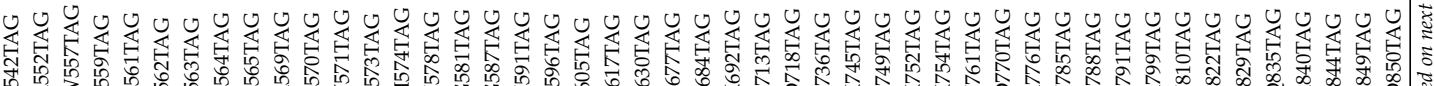

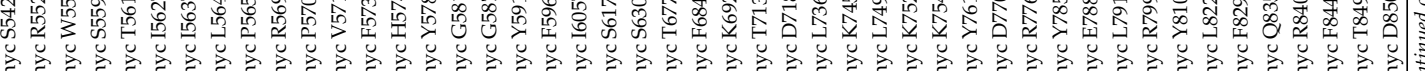
gू

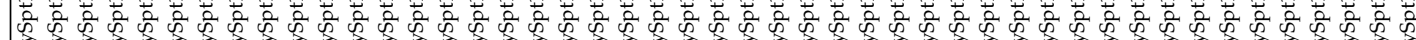

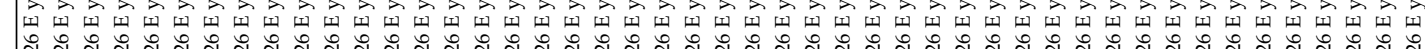

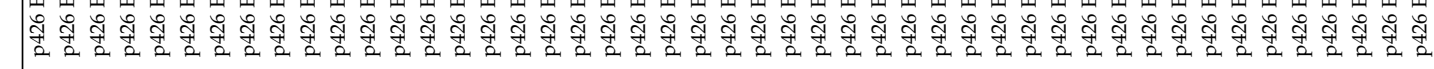

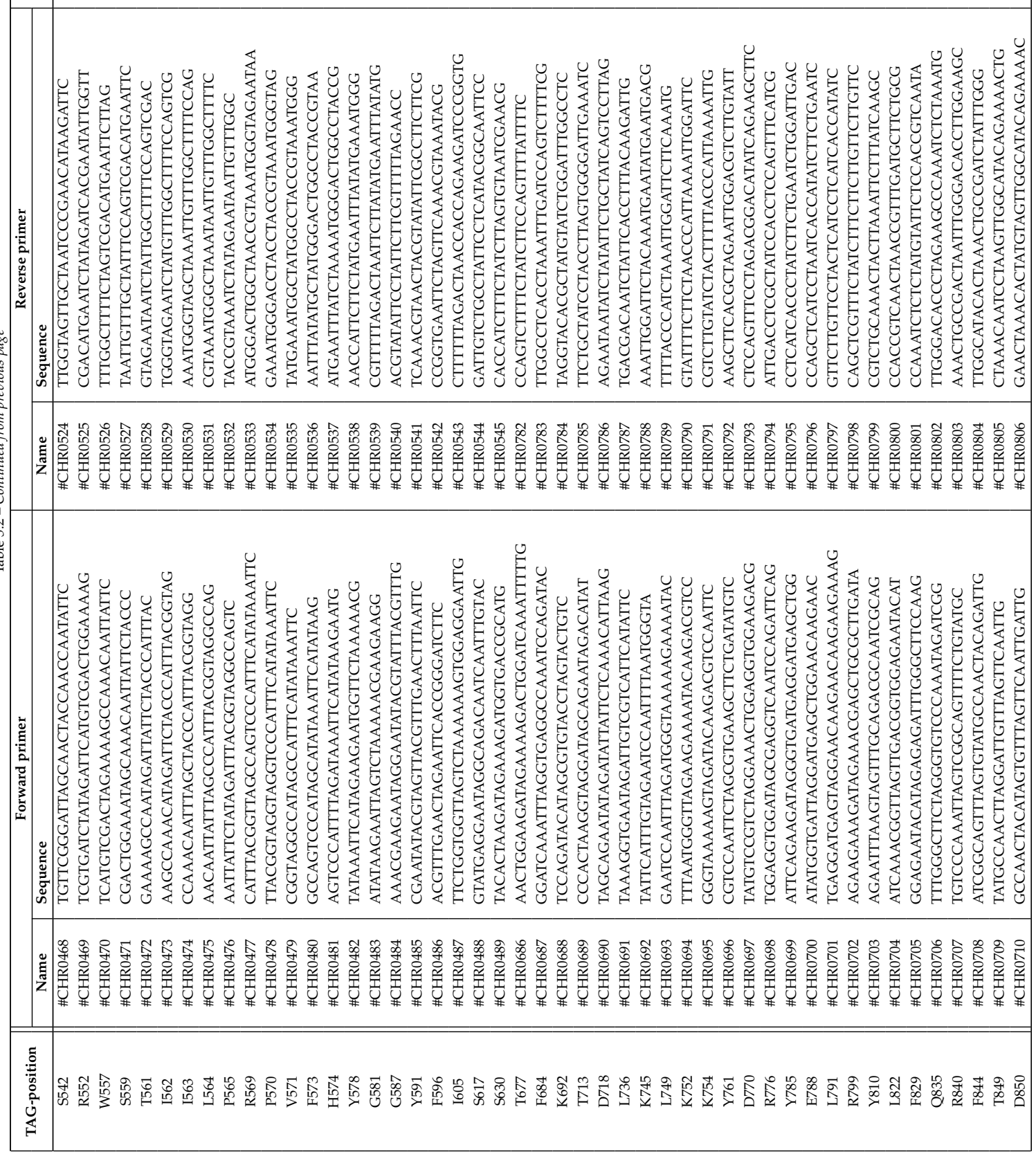




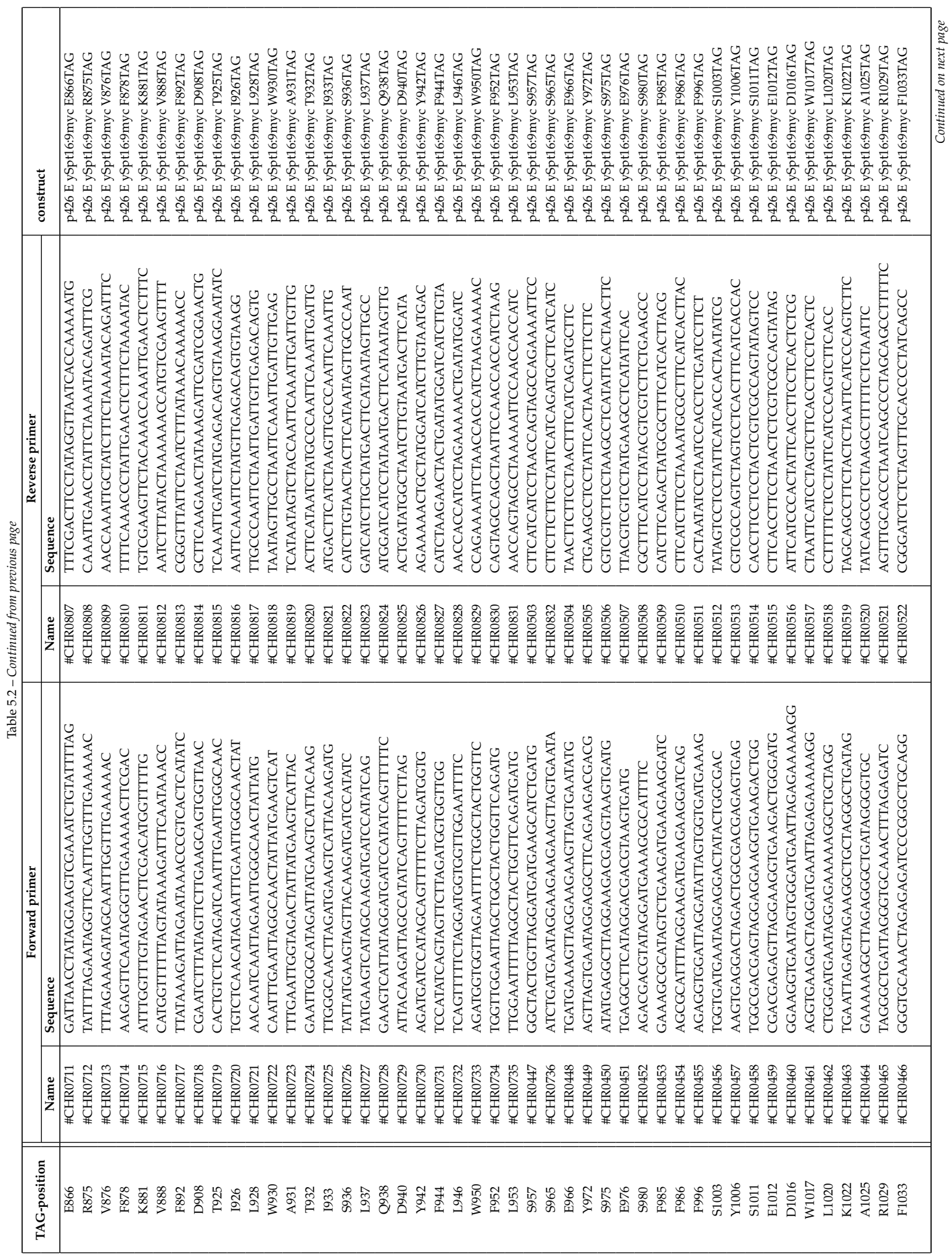




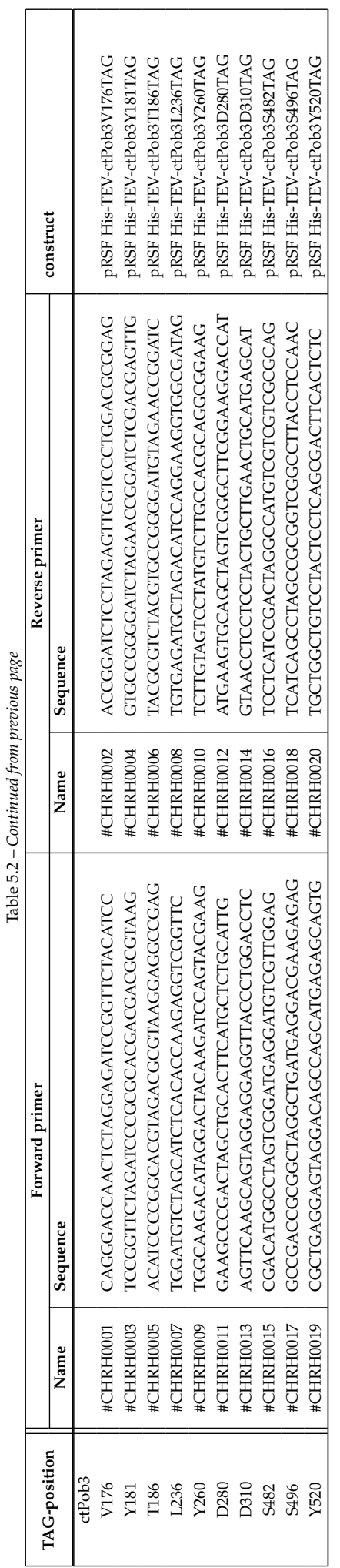




\section{Appendix}

Table 5.3: Classification of the pBPA-dependent rescue assay for Spt16-pBPA mutants classification refers to the plating assay shown in figure 3.9 and 3.10

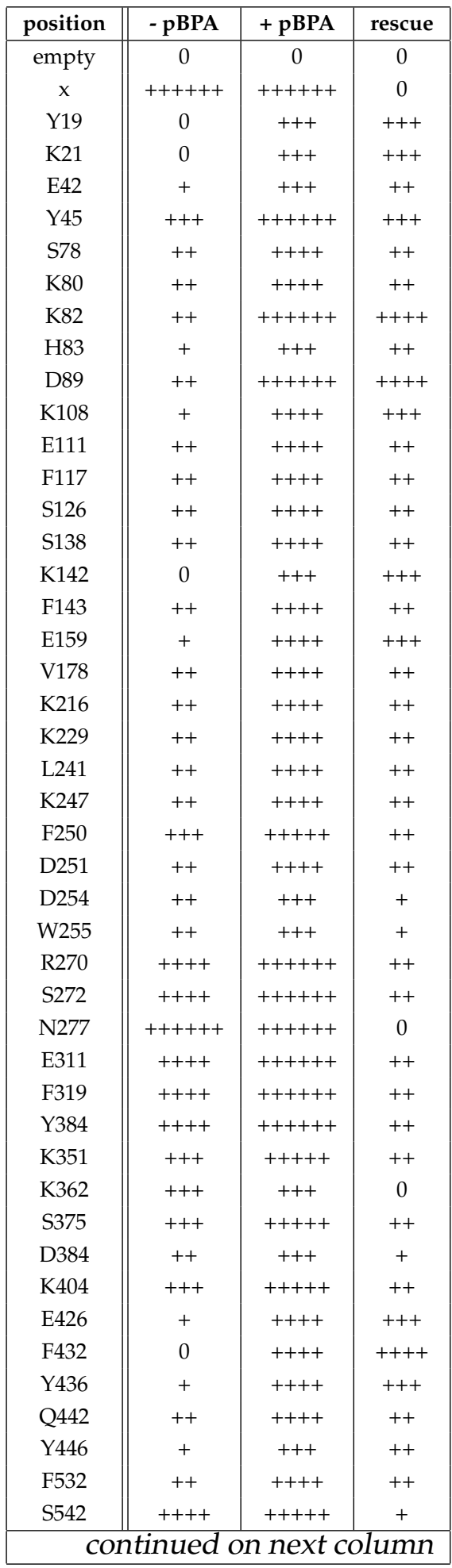

\begin{tabular}{|c|c|c|c|}
\hline position & - pBPA & + pBPA & rescue \\
\hline R552 & + & ++++ & +++ \\
\hline W557 & ++ & ++++ & ++ \\
\hline S559 & ++++++ & ++++++ & 0 \\
\hline T561 & ++ & +++ & + \\
\hline I562 & + & + & 0 \\
\hline L564 & + & +++++ & ++++ \\
\hline R569 & + & +++++ & ++++ \\
\hline P570 & +++ & ++++++ & +++ \\
\hline V571 & 0 & 0 & 0 \\
\hline F573 & ++ & ++++ & ++ \\
\hline H574 & + & ++++ & +++ \\
\hline Y578 & ++ & ++++ & ++ \\
\hline Y591 & ++ & ++++ & ++ \\
\hline F596 & ++ & ++++ & ++ \\
\hline I605 & + & ++++ & +++ \\
\hline S617 & ++++ & +++++ & + \\
\hline T677 & + & + & 0 \\
\hline F684 & + & + & 0 \\
\hline K692 & 0 & 0 & 0 \\
\hline $\mathrm{T} 713$ & 0 & 0 & 0 \\
\hline D718 & 0 & + & + \\
\hline L736 & + & ++++ & +++ \\
\hline K745 & + & ++++ & +++ \\
\hline L749 & + & +++ & ++ \\
\hline K752 & + & +++ & ++ \\
\hline K754 & +++ & +++++ & ++ \\
\hline Y761 & + & ++++ & +++ \\
\hline D770 & + & ++++ & +++ \\
\hline R776 & + & ++++ & +++ \\
\hline Y785 & + & ++++ & +++ \\
\hline E788 & + & ++++ & +++ \\
\hline L791 & + & ++++ & +++ \\
\hline R799 & 0 & ++++ & ++++ \\
\hline Y810 & + & + & 0 \\
\hline L822 & 0 & + & + \\
\hline F829 & 0 & 0 & 0 \\
\hline Q835 & + & + & 0 \\
\hline $\mathrm{R} 840$ & 0 & 0 & 0 \\
\hline F844 & 0 & 0 & 0 \\
\hline T849 & 0 & 0 & 0 \\
\hline D850 & 0 & 0 & 0 \\
\hline E866 & ++++ & ++++++ & ++ \\
\hline R875 & 0 & 0 & 0 \\
\hline V876 & 0 & +++ & +++ \\
\hline F878 & 0 & ++++ & ++++ \\
\hline K881 & 0 & +++ & +++ \\
\hline \multicolumn{4}{|c|}{ continued on next column } \\
\hline
\end{tabular}




\begin{tabular}{|c|c|c|c|}
\hline position & - pBPA & + pBPA & rescue \\
\hline V888 & 0 & 0 & 0 \\
\hline F892 & 0 & 0 & 0 \\
\hline D908 & 0 & ++++ & ++++ \\
\hline T925 & 0 & 0 & 0 \\
\hline I926 & + & + & 0 \\
\hline L928 & 0 & 0 & 0 \\
\hline W930 & 0 & 0 & 0 \\
\hline A931 & 0 & 0 & 0 \\
\hline T932 & 0 & 0 & 0 \\
\hline I933 & 0 & 0 & 0 \\
\hline S936 & 0 & 0 & 0 \\
\hline L937 & 0 & 0 & 0 \\
\hline Q938 & 0 & ++++ & ++++ \\
\hline D940 & + & + & 0 \\
\hline Y942 & 0 & 0 & 0 \\
\hline L946 & 0 & 0 & 0 \\
\hline W950 & 0 & 0 & 0 \\
\hline L953 & +++++ & +++++ & 0 \\
\hline S965 & 0 & 0 & 0 \\
\hline Y972 & 0 & 0 & 0 \\
\hline S975 & +++++ & +++ & 0 \\
\hline E976 & +++++ & +++ & 0 \\
\hline S980 & +++++ & ++++ & 0 \\
\hline F985 & ++++++ & ++++++ & 0 \\
\hline S986 & ++++++ & ++++++ & 0 \\
\hline D996 & ++++++ & ++++++ & 0 \\
\hline S1003 & ++++++ & ++++++ & 0 \\
\hline Y1006 & ++++++ & ++++++ & 0 \\
\hline S1011 & ++++++ & ++++++ & 0 \\
\hline E1012 & ++++++ & ++++++ & 0 \\
\hline D1016 & ++++++ & ++++++ & 0 \\
\hline W1017 & ++++++ & ++++++ & 0 \\
\hline L1020 & ++++++ & ++++++ & 0 \\
\hline K1022 & ++++++ & ++++++ & 0 \\
\hline A1025 & ++++++ & ++++++ & 0 \\
\hline R1029 & +++++ & +++++ & 0 \\
\hline F1033 & +++++ & +++++ & 0 \\
\hline
\end{tabular}

Table 5.4: Classification of the pBPA-dependent rescue assay for Pob3-pBPA mutants classification refers to the plating assay shown in figure 3.9 and 3.11

\begin{tabular}{|c|c|c|c|c|}
\hline position & $25^{\circ} \mathrm{C}+\mathrm{pBPA}$ & - pBPA & $+\mathrm{pBPA}$ & rescue \\
\hline empty & +++++ & 0 & 0 & 0 \\
\hline wt & +++ & ++ & ++ & 0 \\
\hline L10 & +++++ & +++ & ++++ & + \\
\hline R20 & +++++ & ++++ & +++++ & + \\
\hline $\mathrm{I} 30$ & +++++ & ++++ & +++++ & + \\
\hline Q40 & +++++ & ++++ & ++++ & 0 \\
\hline T50 & ++++ & +++++ & ++++ & 0 \\
\hline G60 & ++++ & ++++ & ++++ & 0 \\
\hline $\mathrm{T} 70$ & +++++ & ++++ & ++++ & 0 \\
\hline G80 & +++++ & +++ & ++++ & + \\
\hline K90 & +++++ & +++++ & +++++ & 0 \\
\hline Q100 & +++++ & +++++ & +++++ & 0 \\
\hline G110 & ++++ & ++ & ++++ & ++ \\
\hline R120 & +++++ & +++++ & +++++ & 0 \\
\hline K130 & +++++ & ++++ & +++++ & + \\
\hline I140 & ++++ & +++ & ++++ & + \\
\hline E160 & ++++ & +++++ & ++++ & 0 \\
\hline V170 & ++++ & +++++ & ++++ & 0 \\
\hline I180 & +++ & +++ & +++ & 0 \\
\hline K190 & +++++ & ++++ & ++++ & 0 \\
\hline P200 & ++++ & ++++ & ++++ & 0 \\
\hline D210 & +++++ & ++++ & ++++ & 0 \\
\hline M220 & ++++ & +++ & +++ & 0 \\
\hline E230 & ++++ & ++ & +++ & + \\
\hline D240 & + & 0 & 0 & 0 \\
\hline F250 & + & 0 & 0 & 0 \\
\hline T252 & + & 0 & 0 & 0 \\
\hline R254 & + & 0 & 0 & 0 \\
\hline R256 & + & 0 & 0 & 0 \\
\hline D258 & + & 0 & 0 & 0 \\
\hline D260 & + & 0 & 0 & 0 \\
\hline G270 & ++++ & 0 & ++ & ++ \\
\hline K271 & ++++ & 0 & ++ & ++ \\
\hline T272 & +++ & 0 & + & + \\
\hline Y273 & ++++ & 0 & ++ & ++ \\
\hline E274 & ++++ & 0 & ++ & ++ \\
\hline K276 & + & 0 & 0 & 0 \\
\hline Q278 & + & 0 & 0 & 0 \\
\hline R280 & +++++ & 0 & +++ & +++ \\
\hline K290 & +++++ & ++ & +++ & + \\
\hline V299 & ++++ & ++ & ++++ & ++ \\
\hline L300 & +++++ & ++ & ++++ & ++ \\
\hline E303 & +++++ & ++ & ++++ & ++ \\
\hline Q308 & +++++ & 0 & +++ & +++ \\
\hline Q310 & +++++ & 0 & +++ & +++ \\
\hline Y313 & +++++ & ++ & ++++ & ++ \\
\hline
\end{tabular}




\begin{tabular}{|c||c|c|c|c|}
\hline position & $\mathbf{2 5}$ C + pBPA & p pPPA & + pBPA & rescue \\
\hline F315 & ++++ & 0 & +++ & +++ \\
F320 & ++++ & ++++ & +++++ & 0 \\
K322 & ++++ & + & +++ & ++ \\
D323 & ++++ & + & +++ & ++ \\
E325 & +++ & 0 & + & + \\
L330 & ++++ & + & +++ & ++ \\
N340 & ++++ & 0 & + & + \\
V360 & ++++ & + & ++++ & +++ \\
I370 & + & 0 & 0 & 0 \\
D380 & + & 0 & 0 & 0 \\
A390 & +++ & 0 & 0 & 0 \\
N400 & ++++ & 0 & 0 & 0 \\
K407 & +++++ & 0 & +++ & +++ \\
L410 & +++++ & ++ & +++++ & +++ \\
Y411 & +++++ & ++ & ++++ & ++ \\
V420 & +++++ & + & ++++ & +++ \\
R424 & ++++ & ++ & ++++ & ++ \\
T430 & ++++ & 0 & ++++ & ++++ \\
R433 & ++++ & 0 & ++++ & ++++ \\
D436 & ++++ & 0 & ++++ & ++++ \\
V440 & ++++ & 0 & ++++ & ++++ \\
F450 & ++++ & 0 & +++ & +++ \\
S454 & ++++ & + & ++++ & +++ \\
L460 & ++++ & 0 & +++ & +++ \\
F464 & ++++ & 0 & +++ & +++ \\
L470 & + & 0 & 0 & 0 \\
R471 & + & 0 & 0 & 0 \\
N474 & + & 0 & 0 & 0 \\
Q480 & + & 0 & 0 & 0 \\
D490 & + & 0 & 0 & 0 \\
S491 & + & 0 & 0 & 0 \\
S500 & +++++ & ++++ & ++++ & 0 \\
S507 & +++++ & ++++ & ++++ & 0 \\
E510 & +++++ & ++++ & ++++ & 0 \\
S516 & +++++ & ++++ & ++++ & 0 \\
E520 & +++++ & ++++ & ++++ & 0 \\
F527 & ++++ & +++ & +++ & 0 \\
D530 & +++++ & ++++ & ++++ & 0 \\
S534 & ++++++ & ++++ & ++++ & 0 \\
E537 & ++++++ & ++++ & ++++ & 0 \\
S546 & ++++++ & ++++ & ++++ & 0 \\
K550 & ++++++ & ++++ & ++++ & 0 \\
\hline & & & & \\
\hline
\end{tabular}




\title{
Nomenclature
}

\author{
$\beta \mathrm{ME} \ldots \ldots \ldots \ldots \beta$-mercaptoethanol \\ AARS ......... aminoacyl tRNA synthetase \\ AU $\ldots . . . \ldots . .$. absorbance units \\ BP $\ldots \ldots \ldots \ldots$ band-pass \\ bp ........... base pairs \\ BSA .......... Bovine serum albumin \\ ChIP .......... Chromatin Immunoprecipitation \\ CTD .......... C-terminal domain \\ CV ............ column volume \\ DD .......... dimerization domain \\ dNTP ......... deoxynucleoside triphosphate \\ EMSA $\ldots \ldots \ldots$ electrophoretic mobility shift assay \\ $\mathrm{g} \ldots \ldots \ldots \ldots \ldots$ earth's gravity \\ GCE ........... Genetic code expansion \\ $\mathrm{kb} \ldots \ldots \ldots \ldots$ kilo base pair \\ LB ........... Lysogeny Broth \\ MD ........... middle domain \\ MWCO ........ molecular weight cut off \\ NCP $\ldots \ldots \ldots \ldots$ nucleosome core particle \\ NFR ......... nucleosome-free region \\ NLS ......... nuclear localization signal \\ NTD .......... N-terminal domain \\ ORF $\ldots \ldots \ldots \ldots$ open reading frame \\ pAzF ........ 4-Azido-L-phenylalanine \\ pBPA $\ldots . . \ldots . .4$ 4-Benzoyl-L-phenylalanine \\ PCR .......... Polymerase Chain Reaction \\ $\mathrm{PH} \ldots \ldots \ldots \ldots$. Pleckstrin homology \\ PMT ......... photomultiplier tube \\ PTM .......... post-translational modification \\ $\mathrm{rpm} \ldots \ldots \ldots \ldots$ revolutions per minute \\ RT $\ldots \ldots \ldots \ldots$ room temperature
}


SDS-PAGE ..... Sodium dodecyl sulfate polyacrylamide gel electrophoresis

SEC .......... size exclusion chromatography

SILAC ........ stable isotope labeling by amino acids in cell culture

TB .......... Tris-borate buffer

TBE .......... Tris-borate EDTA buffer

TCA .......... Trichloroacetic acid

U ............ unit

UAA .......... unnatural amino acid

$\mathrm{v} / \mathrm{v} \ldots \ldots \ldots . .$. volume per volume

$\mathrm{w} / \mathrm{v} \ldots \ldots \ldots$ weight per volume

wt $\ldots \ldots \ldots \ldots$ wild-type 


\section{List of Figures}

1.1 Schematic illustration of the DNA by James Watson (taken from Klug,

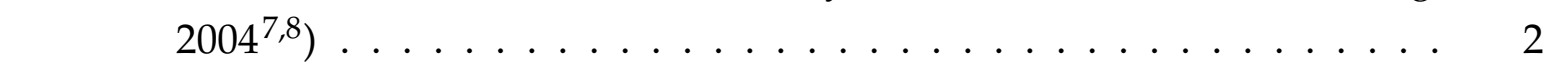

1.2 Schematic illustration of the hierarchical DNA compaction (taken from Tamara L. Caterino and Jeffrey J. Hayes, 200714 $)$. . . . . . . . . . . . 3

1.3 Histones organization and the nuclear core particle (modified after Luger et al., 2001 $\left.{ }^{18}\right)$. . . . . . . . . . . . . . . . . 4

1.4 Nucleosome occupancy at genes in yeast (modified after Jiang and Pugh et al., 2009 22 , reproduction 23 ) . . . . . . . . . . . . . 6

1.5 Overview of classical histone post-translational modifications in Saccharomyces cerevisiae (modified after ${ }^{55}$ ) . . . . . . . . . . . . . . 9

1.6 Nucleosome dynamics: DNA breathing and alternative nucleosome structures (modified after $\left.\frac{7172 / 7374}{2}\right)$. . . . . . . . . . . . . . . . . . . 11

1.7 The histone chaperone escort pathway with selected structural studies

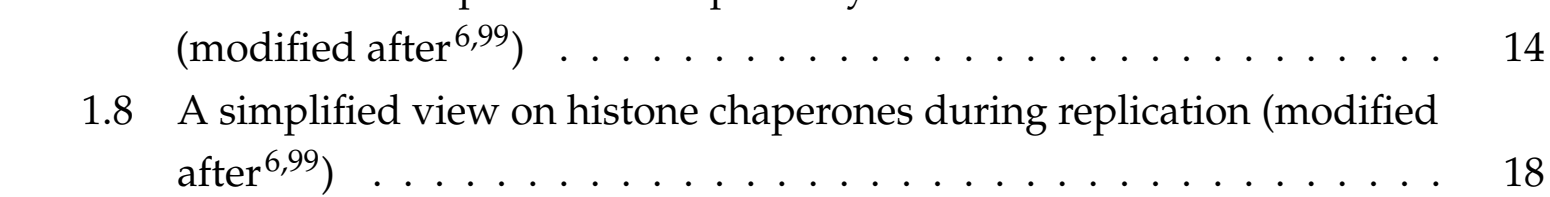

1.9 Histone chaperones during transcription elongation(modified after ${ }^{699}$ ) 19

1.10 Selected structures of FACT domains and models for nucleosome reorganisation (modified after 1531155$)$. . . . . . . . . . . . 22

1.11 Genetically encoded photo-crosslinkers in Saccharomyces cerevisiae . . 33

2.1 Migration patterns of DNA and Protein ladders . . . . . . . . . . 47

2.2 Directional cloning of the terminal Pob3 region (amino acid P305 to

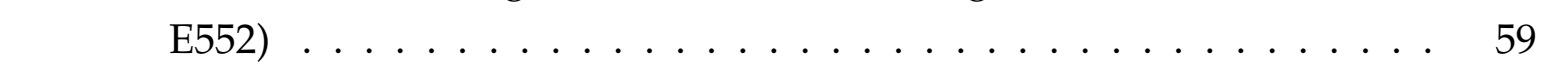

3.1 Detection of plasmid-borne Spt16 and Pob3 by western blot . . . . . 74

3.2 Initial pBPA-crosslinking in Pob3-MD reveal UV-dependent and site-

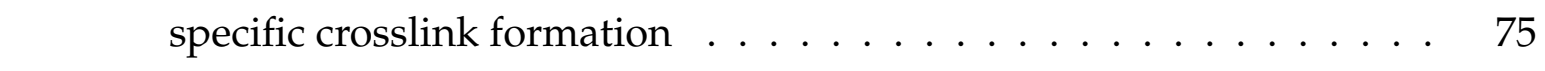

3.3 Influence of UV exposure time on Pob3 S500 crosslink product formation 77

3.4 Comparison between standard chemiluminescent and fluorescent western blot detection ................ 78 
3.5 Comparison among different sample preparation techniques for western blot samples . . . . . . . . . . . . . . . . . . . . . . . 80

3.6 Crosslinking experiments in a protease deficient yeast strain . . . . . . 81

3.7 Scanning of the FACT complex with the genetically encoded UVinducible crosslinker pBPA in Saccharomyces cerevisiae . . . . . . . . 83

3.8 Controls for the FACT complex pBPA scan in Saccharomyces cerevisiae 84

3.9 pBPA-dependent rescue assay testing selected Spt16/Pob3-pBPA mutants for functionality in temperature sensitive yeast strains . . . . . 85

3.10 pBPA-dependent rescue assay testing Spt16-pBPA mutants for functionality in spt16-ts strain . . . . . . . . . . . . . . . . . . 86

3.11 pBPA-dependent rescue assay testing Pob3-pBPA mutants for functionality in pob3-L78R strain . . . . . . . . . . . . . . 8 87

3.12 Molecular weight shift approach and identification of an Pob3-H2A crosslink at serine 500. . . . . . . . . . . . . . . 89

3.13 Subcellular localization and quantification of the Pob3-S500pBPA-H2A

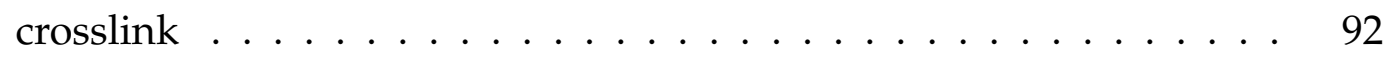

3.14 Investigation of the Pob3-S500 H2A crosslink comparing two different genetically encoded crosslinkers: 4-Benzoyl-L-phenylalanine (pBPA) and 4-Azido-L-phenylalanine (pAzF) . . . . . . . . . . . . . 93

3.15 Scanning of the Pob3-CTD with pBPA for histone interactions in genomically tagged yeast strains . . . . . . . . . . . . . 95

3.16 Scanning of the Spt16-CTD with pBPA for histone interactions in genomically tagged yeast strains . . . . . . . . . . 96

3.17 Scanning of the Pob3-MD basic batch with pBPA for H4 interactions using a genomically tagged H4:3myc yeast strain . . . . . . . . . . 97

3.18 Molecular weight shift experiments of the Spt16-NTD with genomically tagged yeast strains . . . . . . . . . . . . . . 98

3.19 Molecular weight shift experiments of the Spt16 middle domain using genomically tagged yeast strains . . . . . . . . . . . . 99

3.20 Immunoprecipitation of the Pob3:9mycS500-H2A crosslink analyzed by western blot and SDS-PAGE . . . . . . . . . . . . . . . . . . 101

3.21 Protein alignment of Saccharomyces cerevisiae and Chaetomium ther-

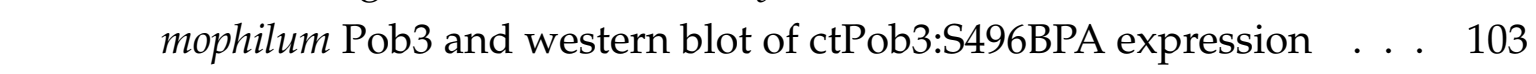

3.22 Size exclusion chromatography analysis of reconstituted Xenopus laevis histone dimers and tetramers and in vitro crosslink analysis of ctPob3 pBPA mutants with histone H2A-H2B dimers . . . . . . . . . . . . 105

3.23 Crosslink experiments of the ctPob3S496pBPA mutant in presence of

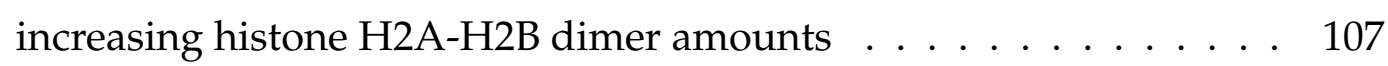


3.24 Purification of yNhp6a from Escherichia coli and binding studies to

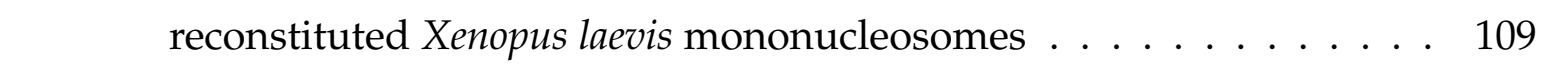

3.25 In vitro crosslink assay of ctPob3S496pBPA with reconstituted dimers and nucleosomes in the presence or absence of yNhp6a . . . . . . . . . 111

3.26 Segment of a protein alignment of 13 fungal Pob3 sequences showing

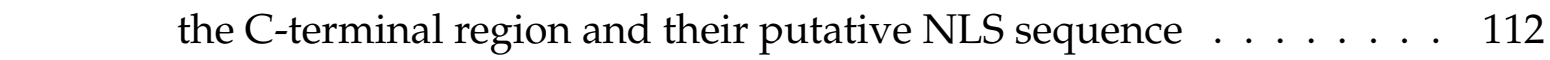

3.27 In vivo localization studies of Pob3:GFP constructs confirming the C-terminal NLS sequence in Saccharomyces cerevisiae . . . . . . . . . . 114

3.28 Saccharomyces cerevisiae and Chaetomium thermophilum Pob3-CTD pro-

tein alignment and SDS-PAGE analysis of recombinantly expressed
importin- $\alpha$ and ctPob3 variants . . . . . . . . . . . . . 116

3.29 Analytical size exclusion chromatography of Chaetomium thermophilum \begin{tabular}{|ll}
\hline Pob3 with recombinantly expressed and purified human importin- $\alpha$ & 117
\end{tabular}

3.30 Competitive pBPA crosslinking assay of ctPob3S496pBPA with importin-

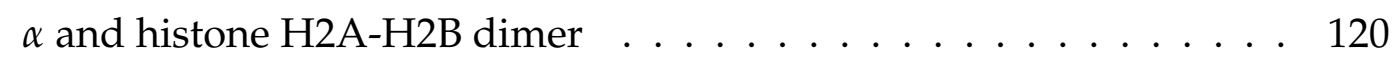

3.31 PCR-based strategy confirming the genomic integration of Pob3-CTD

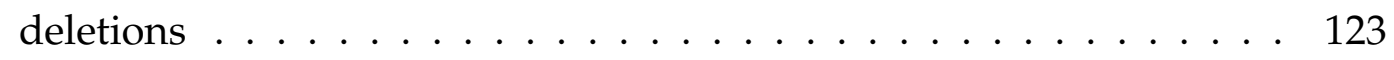

3.32 Spot assay of Pob3-CTD mutant yeast strains under genotoxic or replicative stress induced by hydroxyurea or methyl methanesulfonate 124

4.1 Overview of structural and functional insight of the Pob3-CTD . . . 143 


\section{List of Tables}

2.1 Yeast strains used in this study . . . . . . . . . . . . . . 45

2.2 Antibodies used in this study . . . . . . . . . . . . . . 46

2.3 Pipetting scheme and PCR program for standard PCR amplification. 50

2.4 Pipetting scheme and PCR program for Quikchange reactions using PfuTurbo $^{\mathrm{R}}$ DNA Polymerase . . . . . . . . . . . . . 50

2.5 Pipetting scheme and PCR program for Quikchange reactions using Phusion $^{\mathrm{R}}$ DNA Polymerase . . . . . . . . . . . . . . . . 51

2.6 Pipetting scheme and PCR program of a standard sequencing reaction using the BigDye ${ }^{(R)}$ Terminator v1.1 Cycle Sequencing Kit . . . . . . . 53

2.7 Agarose concentrations and suitable separation lengths used in gelelectrophoresis (modified after ${ }^{287}$ ) . . . . . . . . . . . 54

2.8 Auxotrophy subcloning of the pESC pBPA-RS TRP and pESC pAzF-RS

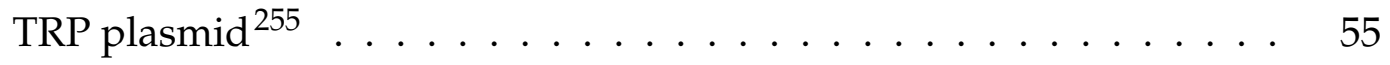

2.9 Cloning strategy for the C-terminal tag vector series . . . . . . . . 56

2.10 Cloning strategy for the parental Spt16 and Pob3 plasmids . . . . . 56

2.11 Intron removal of ctPob3 . . . . . . . . . . . . . . 57

2.12 Construction of ctPob3 amber mutants with mutagenesis PCR primers 58

2.13 NLS constructs for in vivo microscopy analysis . . . . . . . . . . . . 58

2.14 Quikchange mutagenesis strategy for $\Delta$ S491-E543 and $\Delta$ A501-E543

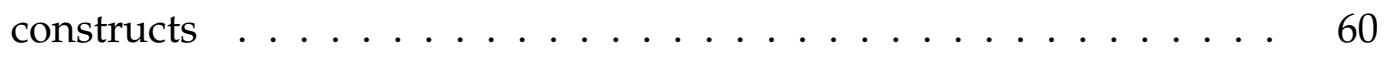

2.15 SDS-PAGE composition . . . . . . . . . . . . . . . . 61

2.16 Molecular weights and molar extinction coefficients $(\epsilon)$ for full-length Xenopus laevis histone proteins (taken from $\left.{ }^{81}\right)$. . . . . . . . . . . . . 67

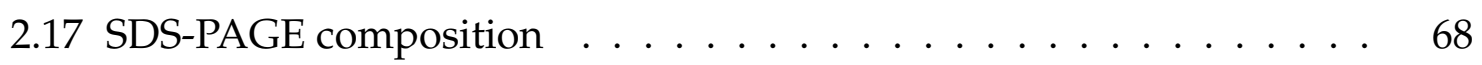

2.18 Pipetting scheme of a standard yeast transformation . . . . . . . . . 69

$3.1 \quad$ Pob3-CTD deletion constructs for genomic integration . . . . . . . . . . 121

5.1 General cloning and sequencing primers . . . . . . . . . . . . . 173

5.2 List of Spt16 and Pob3 amber suppression constructs and their corresponding quikchange mutagenesis primers . . . . . . . . . . 174 
5.3 Classification of the pBPA-dependent rescue assay for Spt16-pBPA mutants - classification refers to the plating assay shown in figure 3.9

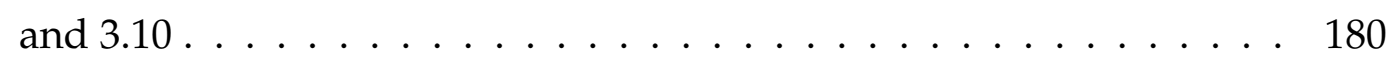

5.4 Classification of the pBPA-dependent rescue assay for Pob3-pBPA mutants - classification refers to the plating assay shown in figure 3.9 and $3.11 \ldots \ldots \ldots \ldots \ldots 18 \ldots \ldots \ldots \ldots$ 


\section{Declaration in Lieu of Oath and Curriculum Vitae}

Herewith I declare, that I prepared the PhD Thesis

"Structural investigation of the histone chaperone complex FACT using genetically encoded crosslinkers in Saccharomyces cerevisiae"

on my own and with no other sources and aids than quoted.

Göttingen, 30. September 2014

(signature) 


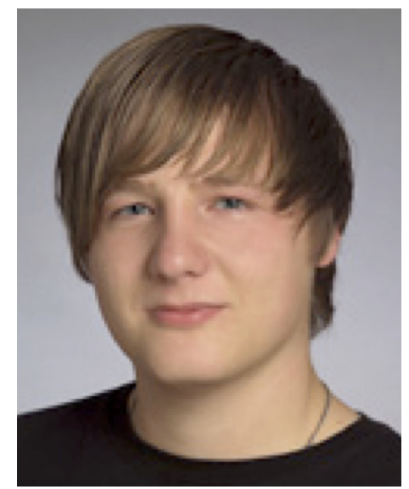

\section{CHRISTIAN HOFFMANN 37073 Göttingen \\ choffmal@gwdg.de}

PERSONAL STATUS: Single

NATIONALITY: Deutsch

BIRTH: 31.05.1986, Altdöbern

CURRENT POSITION PHD STUDENT: PhD Molecular Biology Program

Title: "Structural investigation of the histone chaperone complex FACT using genetically encoded crosslinkers in Saccharomyces cerevisiae" Research group "Applied Synthetic Biology" of Prof. Heinz Neumann Georg August Universität Göttingen / International Max Planck Research School

EDUCATION GÖTTINGEN GRADUATE SCHOOL FOR NEUROSCIENCE, BIOPHYSICS AND MOLECULAR BIOSCIENCES

Göttingen, Niedersachsen - Doctoral candidate, 2011-present (expected graduation date: 12/2014)

GEORG-AUGUST UNIVERSITÄT GÖTTINGEN

Göttingen, Niedersachsen - M.Sc. Molecular Biology, 2009-2011

TECHNISCHE UNIVERSITÄT DRESDEN

Dresden, Sachsen - B.Sc. Molekulare Biotechnologie, 2006-2009

DR. ALBERT SCHWEITZER-GYMNASIUM VETSCHAU

Vetschau, Brandenburg - A-levels, 1998-2005

\section{RELEVANT LAB}

\section{EXPERIENCE MASTER THESIS}

Georg August Universität Göttingen (research group of

Prof. Heinz Neumann - Genetic code expansion - H3K56 acetylation)

New methods to elucidate chromatin assembly

Oct.. 2010 - Mar. 2011

BACHELOR THESIS

BIOTEC TU Dresden (research group of Prof. Gilbert Weidinger - Wnt signaling and regeneration in zebrafish)

Towards molecular insight into transcriptional regulation of trophoblast glycoprotein-like by Wnt/b-catenin signaling in zebrafish

Mar. 2009 - Aug. 2009

Department of Cellular Logistics (Prof. Dirk Görlich),

Max-Planck Institute for biophysical chemistry Göttingen

Construction of FRET-based sensors to probe the interaction of export in 1 with its ligands 
Research group "Applied Synthetic Biology" (Prof. Heinz Neumann)

Georg-August Universität Göttingen

The role of lysine K71 acetylation on the small GTPase Ran

March. 2010 - Apr. 2010

Department of Bioanalytics (Prof. Kai Tittmann)

Göttingen Center for Molecular Biosciences

Expression, purification and characterisation of the E.coli PDH complex

Jun, 2010 - July, 2010

Department of Prof. Dr. Hans Lehrach

Max-Planck Institute for Molecular Genetics Berlin

Cloning of developmental genes and analysis of gene expression in

Nematostella vectensis

Sept. 2007 - Aug. 2008

Student assistant in the research group of Prof. Gilbert Weidinger

BIOTEC TU Dresden

Wnt signaling and regeneration in zebrafish

Sept. 2007 - Feb. 2009

\section{PUBLICATIONS}

Hoffmann C., Neumann H. Acidic CTD of Pob3 interacts with H2A/H2B dimer in vivo, manuscript in preparation

Currently, I am finishing my manuscript that provides an in vivo structural analysis of chromatin remodelling FACT complex. Using unnatural amino acids, I developed an assay to capture protein-protein interactions in bakers yeast, analysing approximately 200 different sites in the FACT complex and checked their individual site-specific crosslinking behavior using the photoreactive crosslinker PBPA.

Kagermeier-Schenk, B., Wehner, D., Ozhan-Kizil, G., Yamamoto, H., Li, J., Kirchner, K., Hoffmann, C., Stern, P., Kikuchi, A., Schambony, A., et al. Waif1/5T4 Inhibits Wnt/B-Catenin Signaling and Activates Noncanonical Wnt Pathways by Modifying LRP6 Subcellular Localization. Dev. Cell, 21: 1129-43 (2011)

For this paper I performed in situ hybridisations and characterised that Waif 1 expression is directly regulated by $\mathrm{Wnt} / \mathrm{B}$-Catenin signaling

\section{AWARDS MAX PLANCK STIPEND FOR THE ADVANCEMENT OF SCIENCE}

For Master Studies: October 2009-March 2010

IGEM Gold medal 2012

Gold medal holder 2012 as Instructor of the iGEM Team "Homing Coli" Göttingen

Göttingen Graduate School for Neuroscience, Biophysics and Molecular Biosciences travel grants: EMBO Conference 2013 and Gordon Research Conference 2014 


\section{TEACHING EXPERIENCE GEORG-AUGUST UNIVERSITÄT GÖTTINGEN}

1. Supervision of three lab rotation students ( 2 month each)

2. Tutor at the course "Enzyme kinetics" for Biochemistry majors Academic years: $2010 / 11 ; 2011 / 12$ and $2012 / 13$

3. Tutor at the course "Redox reactions" for Biochemistry majors Academic years: $2010 / 11 ; 2011 / 12$ and $2012 / 13$

4. Tutorial "Protein Structure and Folding" for students of the Max-Planck M.Sc./PhD program "Molecular Biology"

Academic years: $2010 / 11 ; 2011 / 12$ and $2012 / 13$

\section{CONFERENCES GORDON RESEARCH CONFERENCE "CHROMATIN STRUCTURE AND} FUNCTION"

Bentley University, Waltham, MA USA

poster presentation - "In vivo structural investigation of the histone chaperone complex FACT"

participant in 2014 - 08.06.-13.06.14

EMBO "CHROMATIN AND EPIGENETICS"

EMBL Heidelberg, Germany poster presentation - "Structural investigation of the FACT complex" participant in $2013-08.05 .-12.05 .13$

HORIZONS IN MOLECULAR BIOLOGY

Georg August Universität Göttingen

7th, 9th \& 10th International PhD Student Symposium 'Horizons in Molecular Biology' participant in 2010, 2012, 2013 - 4-day symposium organised by PhD students

Organizer of the 8th International PhD Student Symposium 'Horizons in Molecular Biology' 14.09.-17-09.2011

Organizing period: 10/2010 - 9/2011

SKILLS COMPUTER

- Linux

- scripting language: python

- PyMOL/coot

- SigmaPlot®

- LaTeX

\section{LANGUAGES}

- German (Native)

- English (Proficient)

- Russian (Intermediate)

SOFT-SKILLS

- "Balancing work and life", 2013

- "SciencePlus-Workshop: research funding", 2013

- "Grant writing for scientists", 2014

- "Presentation technique workshop", 2014 


\section{COMMUNITY SERVICE SYNTHETIC BIOLOGY - IGEM}

Instructor of the iGEM-Team Göttingen 2012 "Homing Coli"

Participation at the iGEM 2012 Teachers' Workshop in Paris - 03.06.-04.06.12

\section{COMMUNITY OF INTEREST}

former member of: Interessengemeinschaft für Respekt und gegen Gewalt organisation of sport and music events

\section{OTHER INTERESTS}

- Musical Instruments: Piano, Guitar and Saxophone

- Photography 